Supplement of Eur. J. Mineral., 32, 89-98, 2020

https://doi.org/10.5194/ejm-32-89-2020-supplement

(C) Author(s) 2020. This work is distributed under

the Creative Commons Attribution 4.0 License.

(c) (1)
European Journal of

Mineralogy

Open Access

Supplement of

\title{
Halilsarpite, a new arsenate analogue of walentaite, from the Oumlil mine, Bou Azzer district, Morocco
}

Tomas Husdal et al.

Correspondence to: Ian E. Grey (ian.grey@csiro.au)

The copyright of individual parts of the supplement might differ from the CC BY 4.0 License. 


\section{\# 1. PROCESSING SUMMARY (IUCr Office Use Only)}

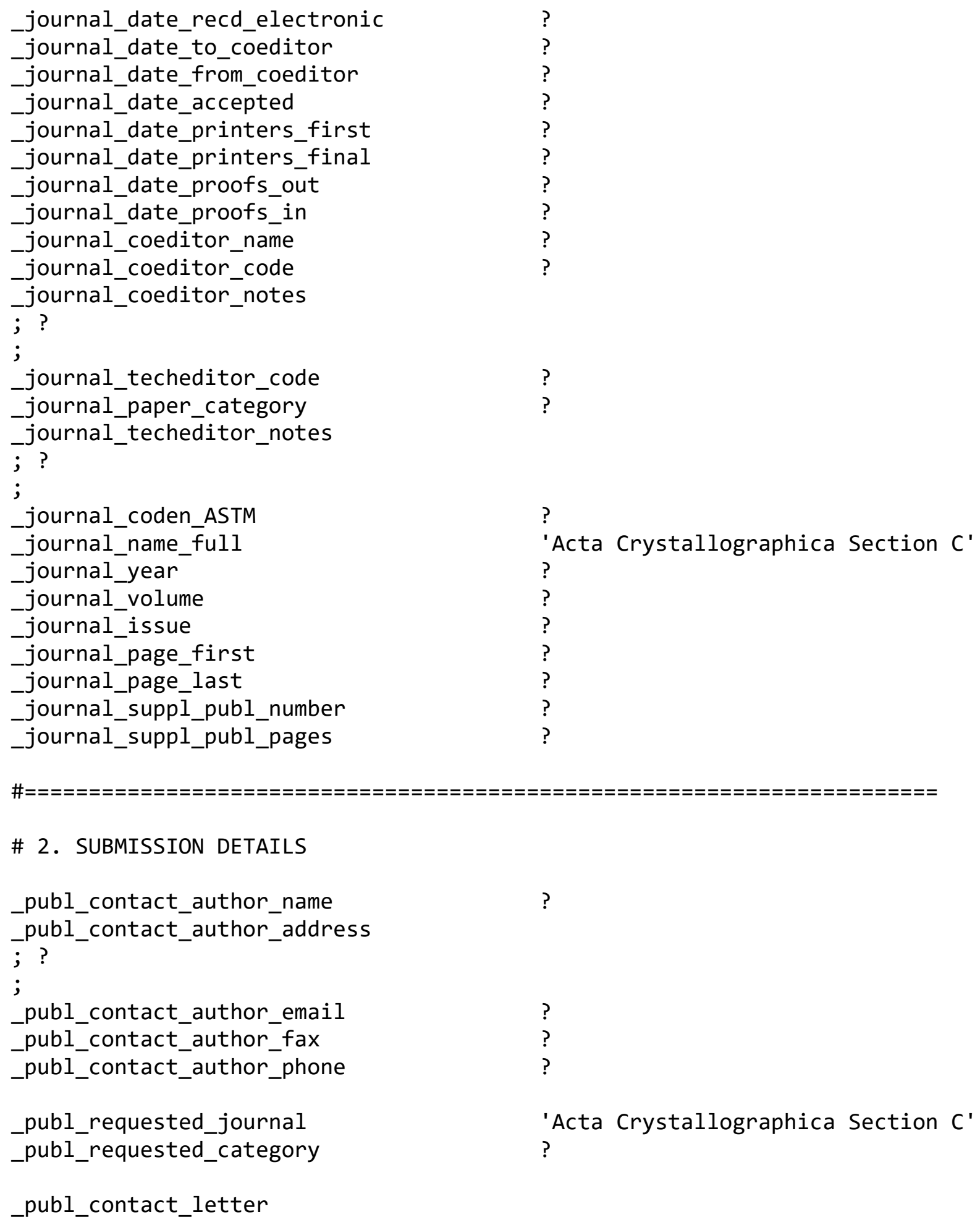




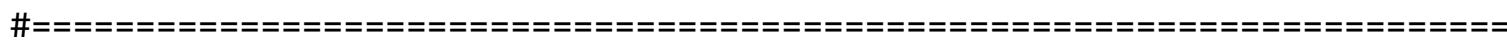

\# 3. TITLE AND AUTHOR LIST

_publ_section_title

; ?

;

_publ_section_title_footnote

; ?

;

loop

_publ_author_name

_publ_author_footnote

_publ_author_address

; ? \# name

;

; ? \# footnote

;

; ? \# address

;

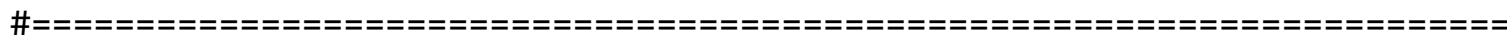

\# 4. TEXT

_publ_section_synopsis

; ?

;

_publ_section_abstract

; ?

;

_publ_section_comment

; ?

;

_publ_section_introduction

; ?

;

_publ_section_experimental

; ?

;

_publ_section_exptl_prep

; ?

;

_publ_section_exptl_refinement

; ?

;

_publ_section_exptl_solution

; ?

;

_publ_section_discussion

; ? 
\# use this reference if SIR2011 was used for solving of the structure \#Burla, M.C., Caliandro, R., Camalli, M., Carrozzini, B., Cascarano, \#G.L., Giacovazzo, C., Mallamo. M., Mazzone, A., Polidori, G., Spagna, R. \#SIR2011: a new package for crystal structure determination and refinement, \#J.Appl.Cryst. (2012) 45, 357-361

\# enable this reference if bond valences were calculated \#Brown, I. D. (1996). J. Appl. Cryst. 29, 479-480.

\# enable this reference if Xshape wase used for crystal shape refinement \#Stoe \& Cie (1998). X-SHAPE. Stoe \& Cie, Darmstadt, Germany.

\# enable this reference if Flack coefficient was refined \#Flack, H. D. (1983). Acta Cryst. A39, 876-881.

; _publ_section_figure_captions 
data_I

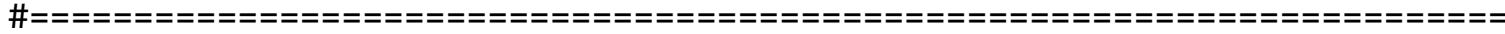

\# 5. CHEMICAL DATA

_chemical_name_systematic

; ?

;

_chemical_name_common

_chemical_formula_moiety

?

_chemical_formula_structural

?

_chemical_formula_analytical

chemical_formula_iupac

?

chemical_formula_sum

'As4.02 Ca0.69 Fe2.55 H12.1 K0.01 Mg0.62 Mo0.56 Na0.11 021 P0.03'

chemical_formula_weight

892.1

_chemical_melting_point

?

chemical_compound_source

_chemical_absolute_configuration

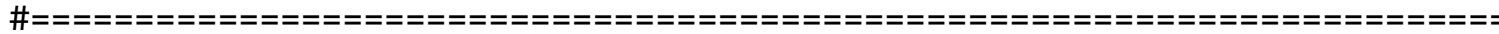

\# 6. CRYSTAL DATA

_symmetry_cell_setting

_symmetry_space_group_name_H-M

_symmetry_space_group_name_Hall

_symmetry_Int_Tables_number

loop_

_space_group_symop_id

_space_group_symop_operation_xyz

$1 \quad x, y, z$

$2-x,-y+1 / 2, z$

$3-x, y+1 / 2,-z$

$4 x,-y,-z$

$5-x,-y,-z$

$6 x, y+1 / 2,-z$

$7 \quad x,-y+1 / 2, z$

$8-x, y, z$

$9 x+1 / 2, y+1 / 2, z+1 / 2$

$10-x+1 / 2,-y, z+1 / 2$

$11-x+1 / 2, y,-z+1 / 2$

$12 x+1 / 2,-y+1 / 2,-z+1 / 2$

$13-x+1 / 2,-y+1 / 2,-z+1 / 2$

$14 x+1 / 2, y,-z+1 / 2$

$15 x+1 / 2,-y, z+1 / 2$

$16-x+1 / 2, y+1 / 2, z+1 / 2$

_cell_length_a

_cell_length_b

_cell_length_c

_cell_angle_alpha

_cell_angle_beta orthorhombic

'I $\mathrm{m} \mathrm{m}$ a'

'- I -2x;-2yb;-2zb'

74
$26.4890(10)$

$7.4205(3)$

$10.4378(4)$

90

90 
loop_

twin_individual_id

_twin_individual_mass_fraction_refined

_twin_individual_twin_matrix_11

_twin_individual_twin_matrix_12

_twin_individual_twin_matrix_13

_twin_individual_twin_matrix_21

_twin_individual_twin_matrix_22

_twin_individual_twin_matrix_23

_twin_individual_twin_matrix_31

_twin_individual_twin_matrix_32

_twin_individual_twin_matrix_33

? ? ? ? ? ? ? ? ? ? ?

_cell_formula_units_z

4

_cell_measurement_reflns_used

_cell_measurement_theta_min

3520

_cell_measurement_theta_max

2

_cell_measurement_temperature

30

_cell_special_details

293

; ?

;

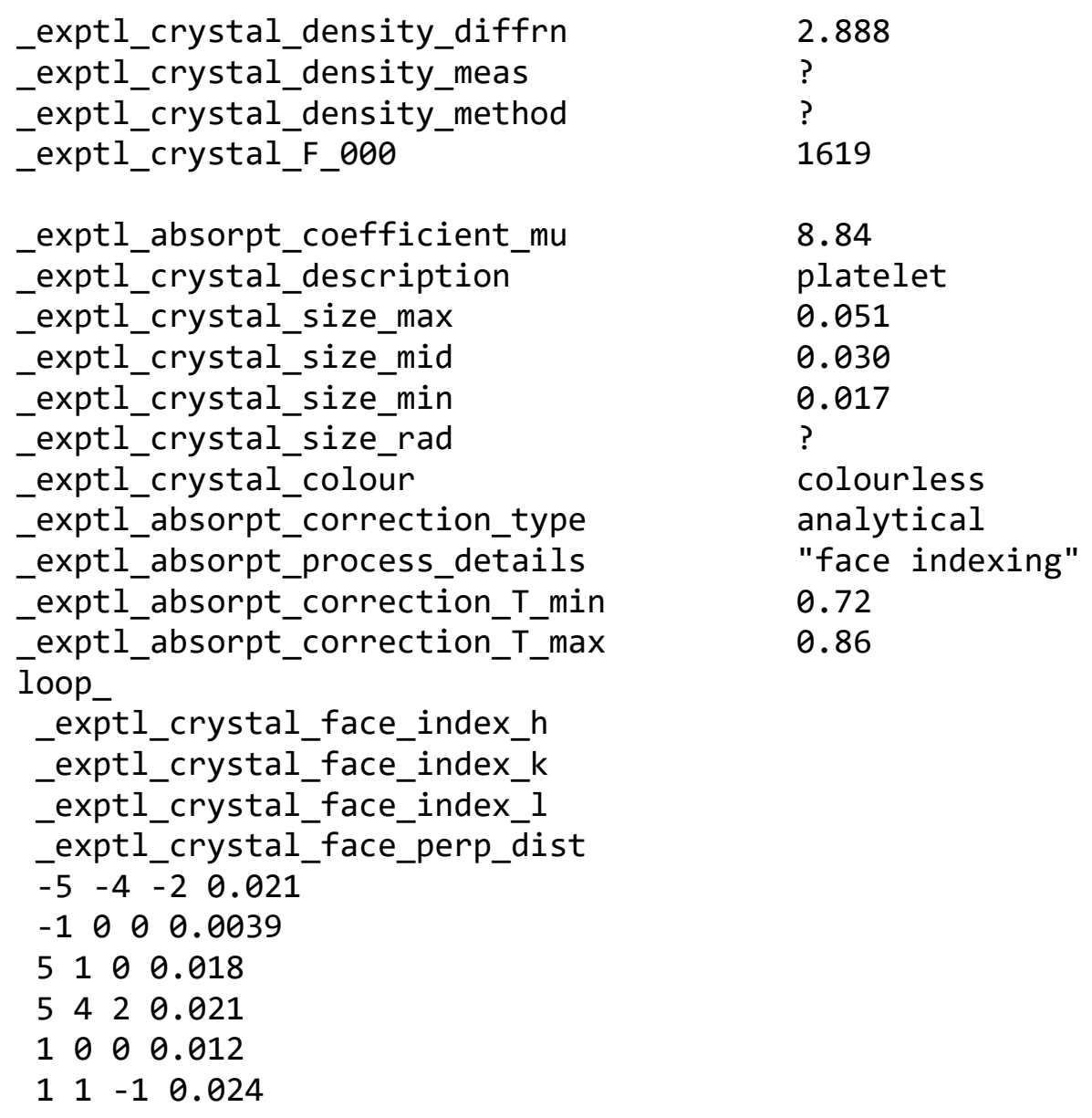


\# 7. EXPERIMENTAL DATA

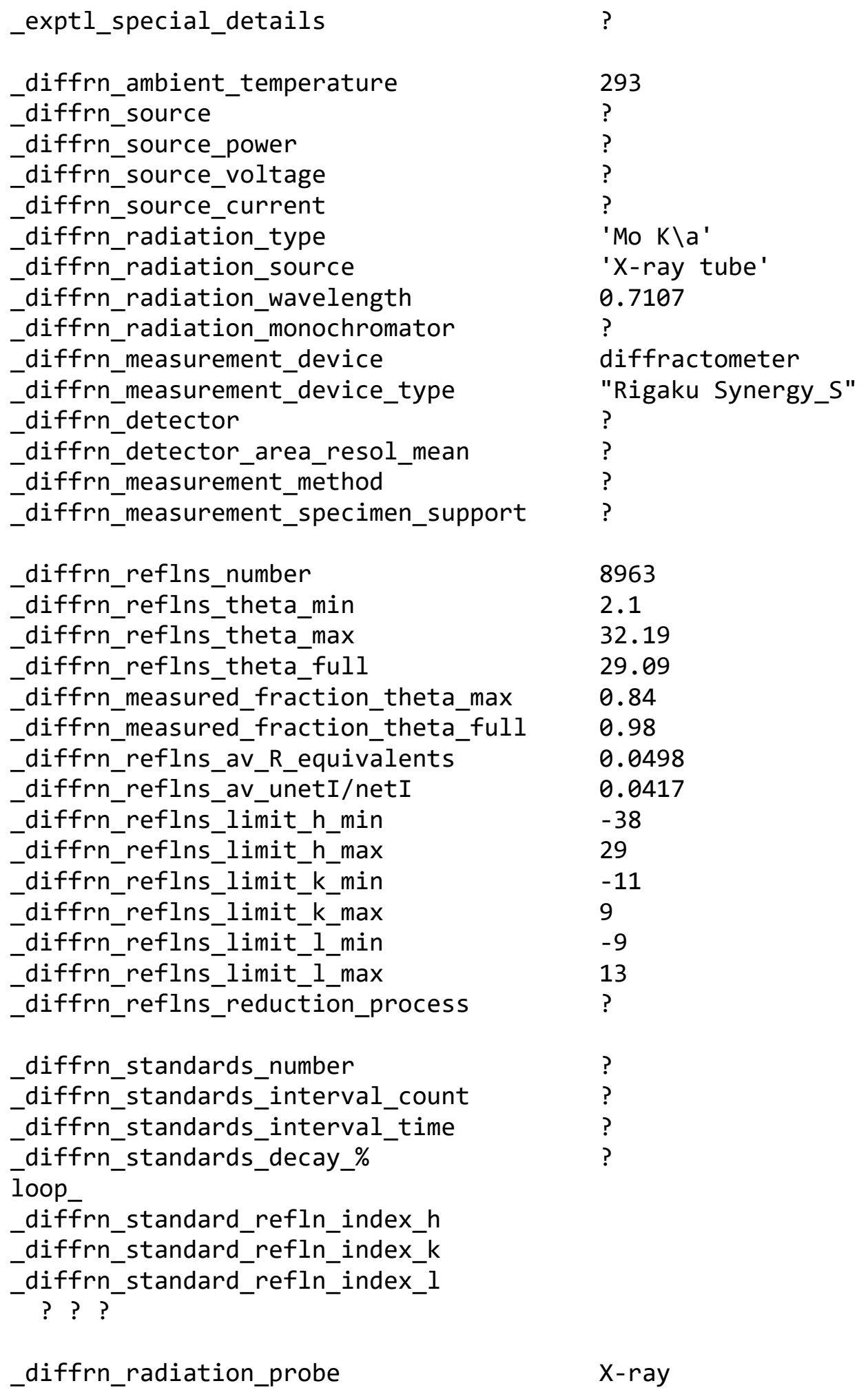


\# 8. REFINEMENT DATA

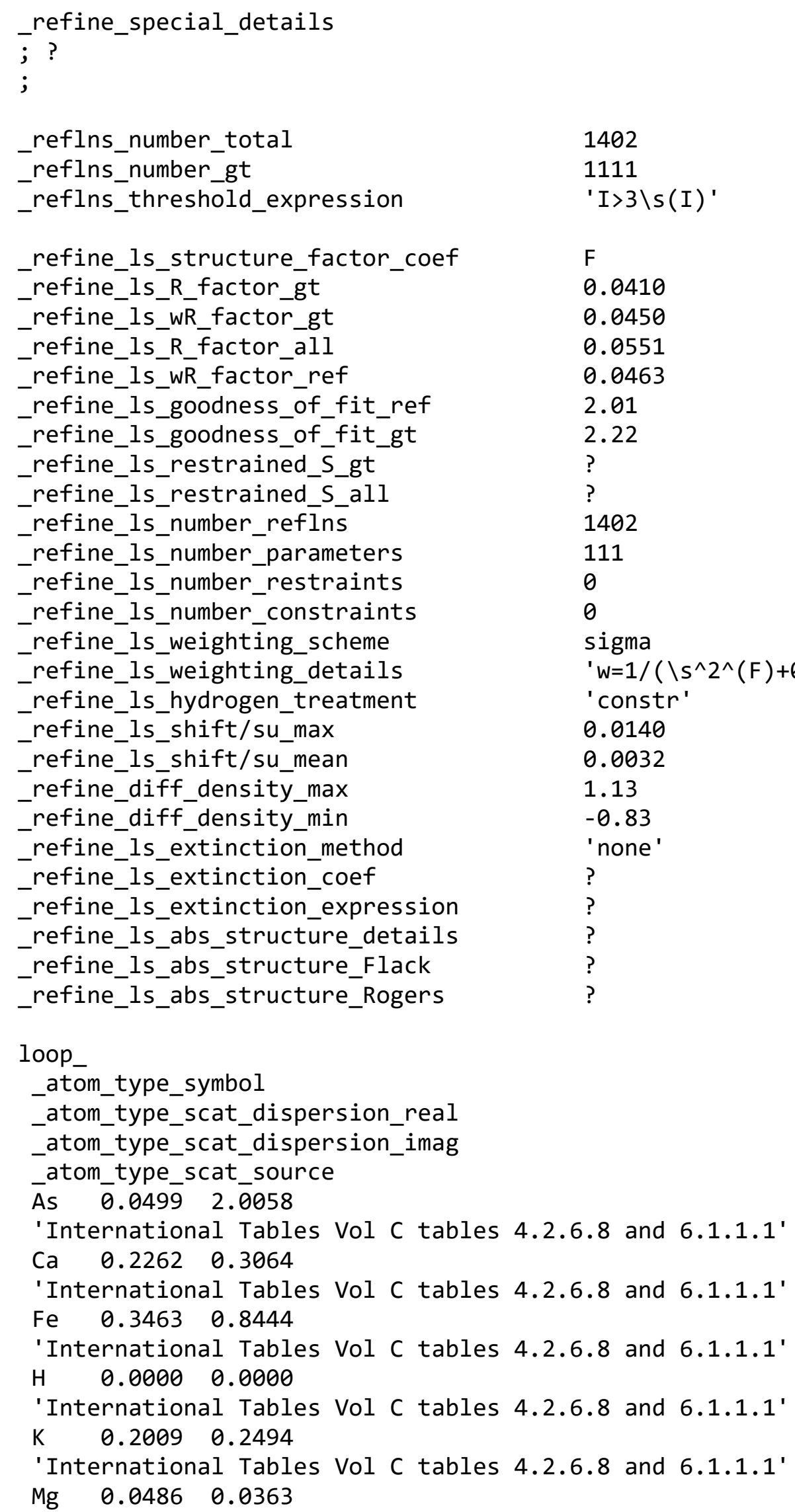




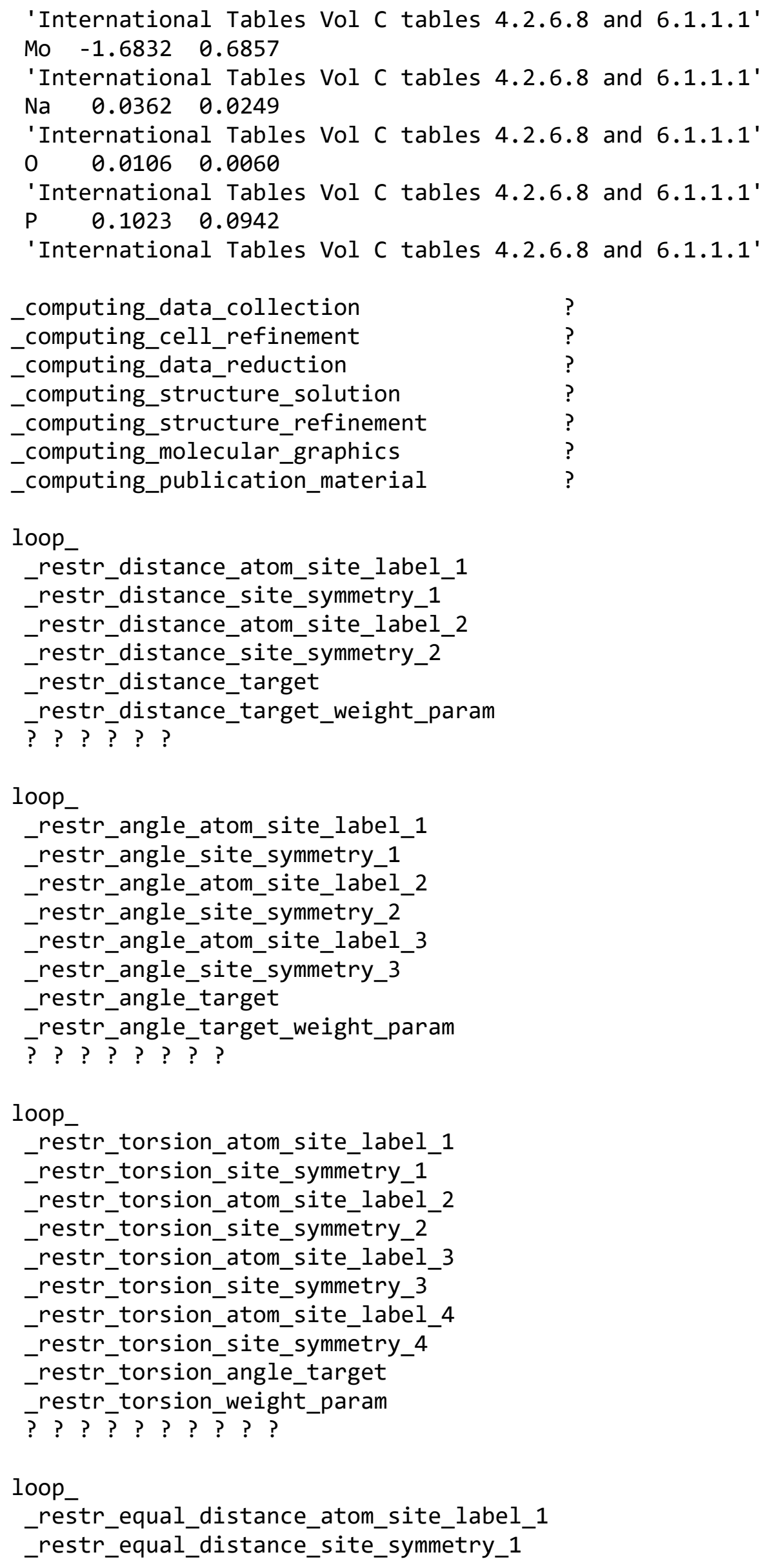




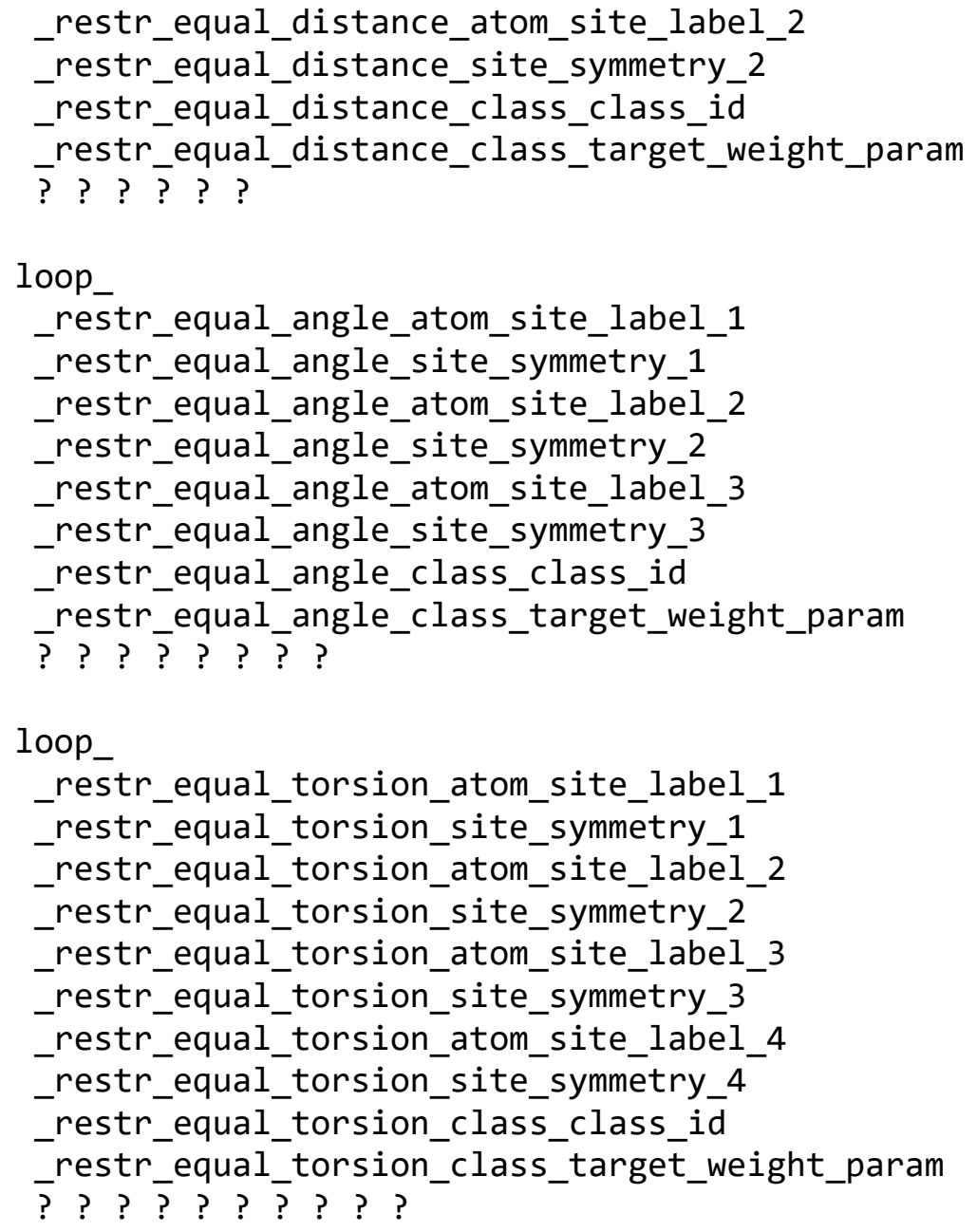

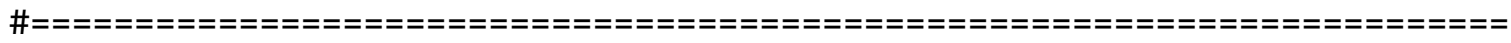

\# 9. ATOMIC COORDINATES AND DISPLACEMENT PARAMETERS

loop

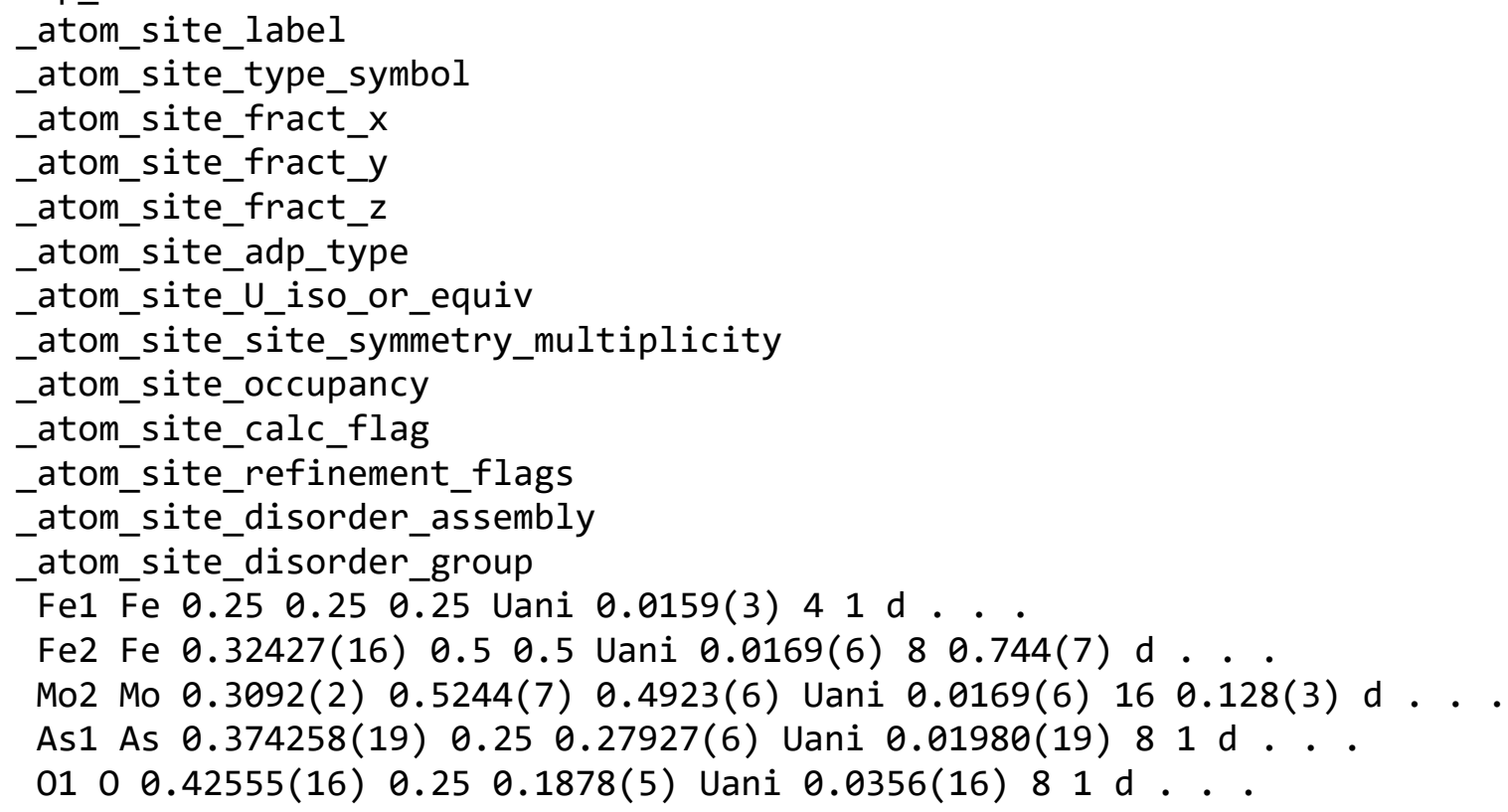


$0200.32184(15) \quad 0.250 .1890(4)$ Uani $0.0317(15) 81 \mathrm{~d} . .$. $0300.37434(10) 0.4339(4) 0.3738(3)$ Uani $0.0270(10) 161 \mathrm{~d} . .$. 04a $00.2684(3) 0.5457(9) 0.6298(7)$ Uiso $0.0179(9) 160.5 \mathrm{~d}$. . . 04b $00.2618(2) 0.5736(10) 0.6072(7)$ Uiso $0.0179(9) 160.5 \mathrm{~d} . .$. $0500.31916(14) 0.250 .5719(4)$ Uani $0.0227(13) 81 \mathrm{~d} . .$. As2 As $0.21445(7) 0.0300(4) 0.5153(4)$ Uani $0.0171(11) 160.242(2) d$. . . As3 As $0.27130(6) 0.3064(2) 0.69453(17)$ Uani $0.0184(6) 160.234(2) \mathrm{d} .$. $0600.2148(3) 0.250 .6021(9)$ Uiso $0.036(3) 80.538(18) \mathrm{d} .$. Fe3 Fe $0.5 \quad 0.750 .3017(7)$ Uiso $0.0429(14) 40.16 \mathrm{~d}$. . . Mg3 Mg $0.5 \quad 0.750 .3017(7)$ Uiso $0.0429(14) 40.205(13) d . .$. Ca1 Ca $0.5 \quad 0.75 \quad 0.1909(9)$ Uiso $0.0429(14) 40.2 d$. . . Mg1 Mg $0.5 \quad 0.75 \quad 0.1909(9)$ Uiso $0.0429(14) 40.172(11) d . .$. Mg2 Mg $0.1831(3) \quad 0.010(3) \quad 0.51$ Uiso $0.0429(14) 160.032(5) \mathrm{d}$. . . Ca2 Ca $0.1831(3) 0.010(3) 0.51$ Uiso $0.0429(14) 160.12 \mathrm{~d}$. . . Ow1a $0 \quad 0.5684(4) \quad 0.750 .3382(16)$ Uiso $0.061(2) 80.578(19) d . .$. Ow1b $0 \quad 0.5814(6) \quad 0.750 .2696(19)$ Uiso $0.061(2) 80.461(18) d . .$. Ow2a $0 \quad 0.5 \quad 0.4645(14) 0.2792(12)$ Uiso $0.061(2) 80.647(18) d . .$. Ow2b $00.50 .524(3) \quad 0.197(2)$ Uiso $0.061(2) 80.358(15) d . .$. Ow3 $00.529(2) \quad 0.750 .482(6)$ Uiso $0.061(2) 80.105(14) d . .$. Ow4 $00.50 .750 .094(5)$ Uiso $0.061(2) 40.20(2) d . .$. Ow5 $00.4475(7) 0.550(3) 0.033(2)$ Uiso $0.061(2) 160.200(10) d . .$. Ow6 $00.3975(11) 0.25-0.042(3)$ Uiso $0.061(2) 80.202(13) d . .$.

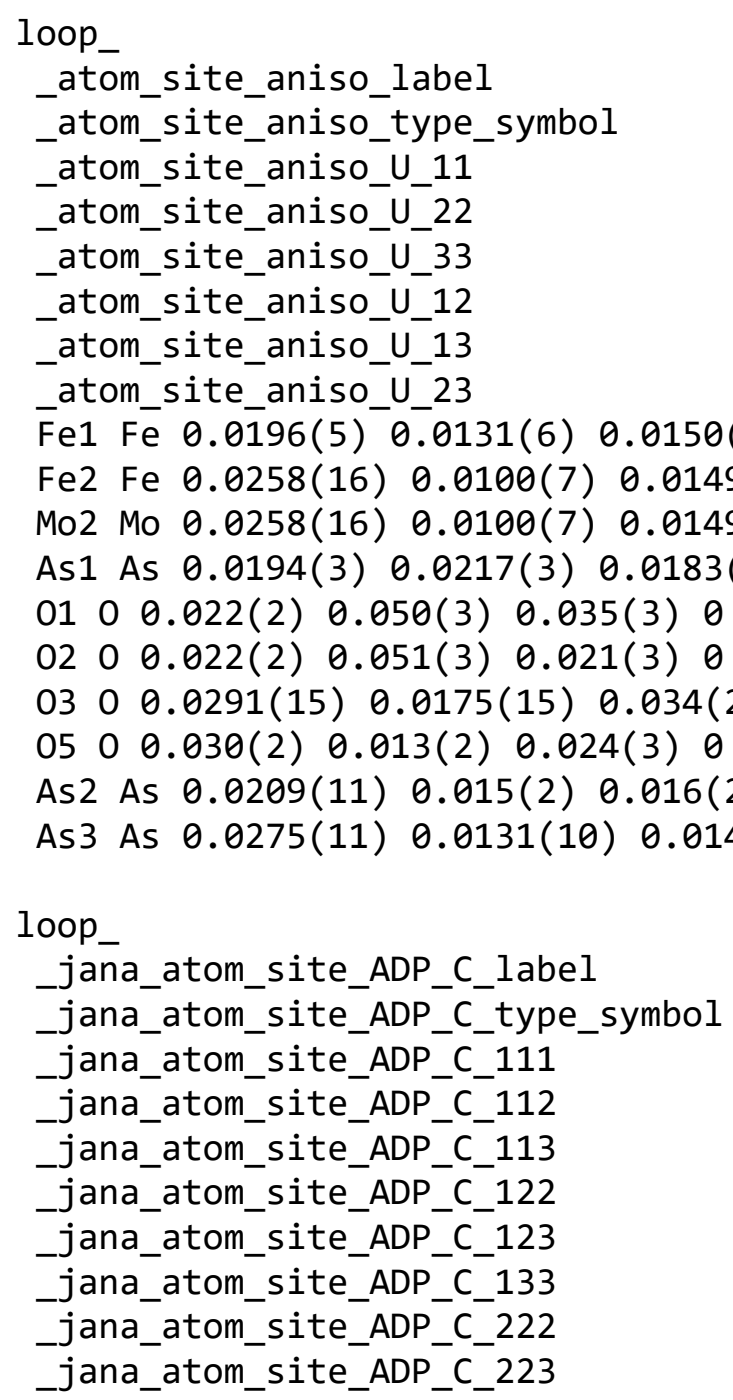


_jana_atom_site_ADP_C_233

_jana_atom_site_ADP_C_333

? ? ? ? ? ? ? ? ? ? ?

loop

_jana_atom_site_ADP_D_label

_jana_atom_site_ADP_D_type_symbol

_jana_atom_site_ADP_D_1111

_jana_atom_site_ADP_D_1112

jana_atom_site_ADP_D_1113

_jana_atom_site_ADP_D_1122

_jana_atom_site_ADP_D_1123

jana_atom_site_ADP_D_1133

jana_atom_site_ADP_D_1222

jana_atom_site_ADP_D_1223

_jana_atom_site_ADP_D_1233

jana_atom_site_ADP_D_1333

_jana_atom_site_ADP_D_2222

_jana_atom_site_ADP_D_2223

jana_atom_site_ADP_D_2233

jana_atom_site_ADP_D_2333

jana_atom_site_ADP_D_3333

? ? ? ? ? ? ? ? ? ? ? ? ? ? ?

loop

_jana_atom_site_ADP_E_label

_jana_atom_site_ADP_E_type_symbol

jana_atom_site_ADP_E_11111

_jana_atom_site_ADP_E_11112

jana_atom_site_ADP_E_11113

jana_atom_site_ADP_E_11122

jana_atom_site_ADP_E_11123

_jana_atom_site_ADP_E_11133

jana_atom_site_ADP_E_11222

jana_atom_site_ADP_E_11223

jana_atom_site_ADP_E_11233

_jana_atom_site_ADP_E_11333

jana_atom_site_ADP_E_12222

jana_atom_site_ADP_E_12223

jana_atom_site_ADP_E_12233

_jana_atom_site_ADP_E_12333

_jana_atom_site_ADP_E_13333

jana_atom_site_ADP_E_22222

jana_atom_site_ADP_E_22223

jana_atom_site_ADP_E_22233

_jana_atom_site_ADP_E_22333

_jana_atom_site_ADP_E_23333

jana_atom_site_ADP_E_33333

? ? ? ? ? ? ? ? ? ? ? ? ? ? ? ? ? ? ? ? ? ? ?

loop

jana_atom_site_ADP_F_label

_jana_atom_site_ADP_F_type_symbol

_jana_atom_site_ADP_F_111111 


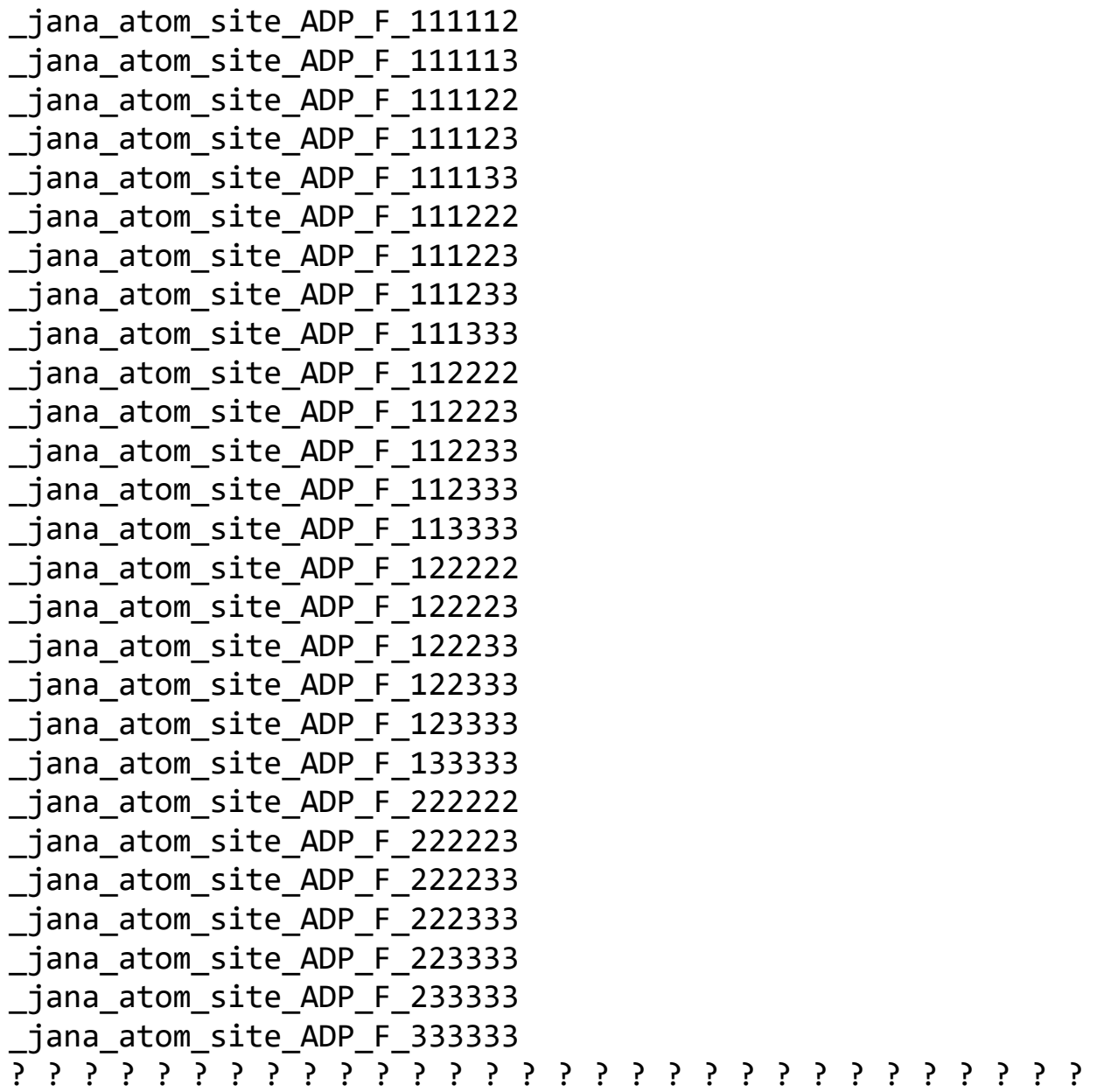

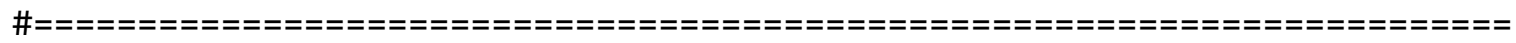

\# 10. MOLECULAR GEOMETRY

loop

_geom_bond_atom_site_label_1

_geom_bond_atom_site_label_2

_geom_bond_site_symmetry_1

_geom_bond_site_symmetry_2

_geom_bond_distance

_geom_bond_publ_flag

Fe1 Fe2 . 3. $758(2)$ ?

Fe1 Fe2 . 10_564 3.758(2) ?

Fe1 Fe2 . 13_555 3.758(2) ?

Fe1 Fe2 . 6_546 3.758(2) ?

Fe1 Mo2 . 3.606(6) ?

Fe1 Mo2 . 10_564 3.535(6) ?

Fe1 Mo2 . 11_555 3.606(6) ?

Fe1 Mo2 . 4_566 3.535(6) ?

Fe1 Mo2 . 13_555 3.606(6) ?

Fe1 Mo2 . 6546 3.535(6) ?

Fe1 Mo2 . 7_555 3.606(6) ?

Fe1 Mo2 . 16_544 3.535(6) ? 


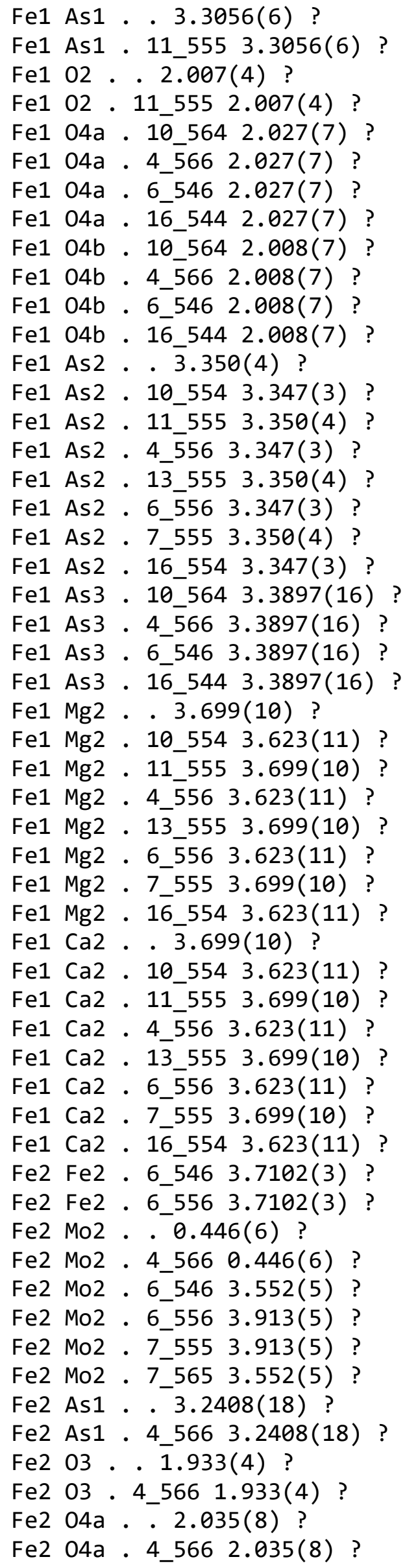




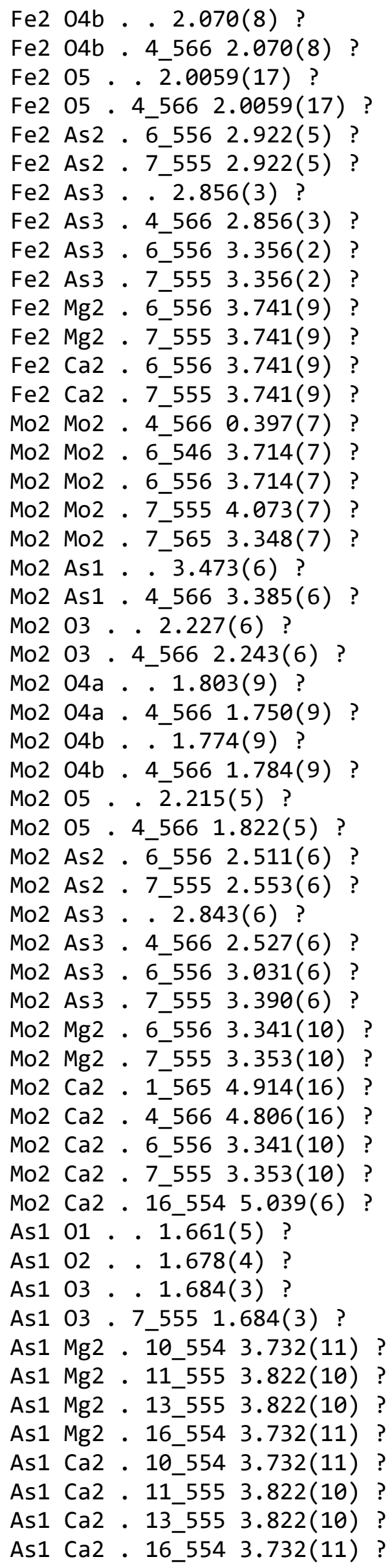




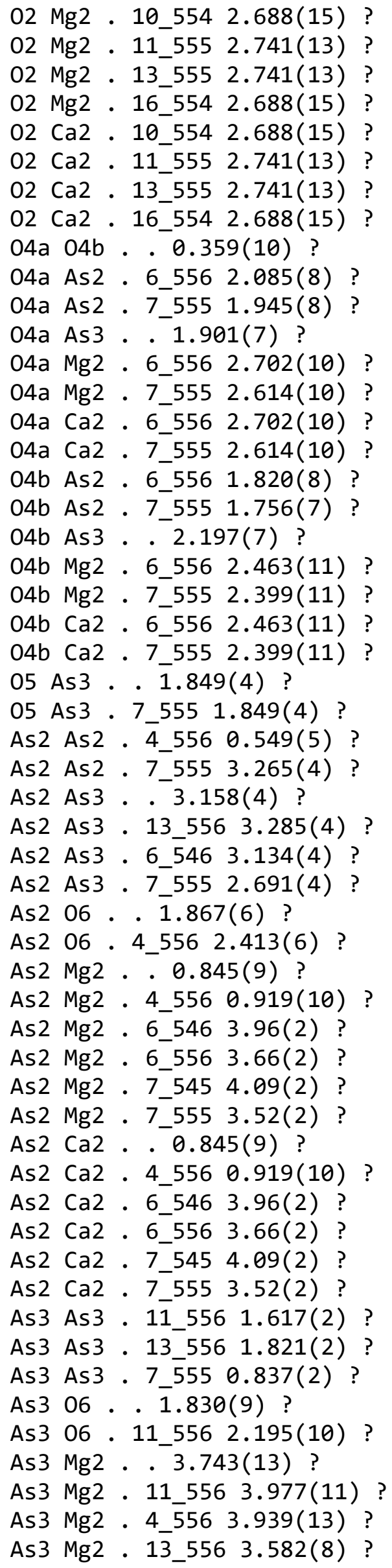




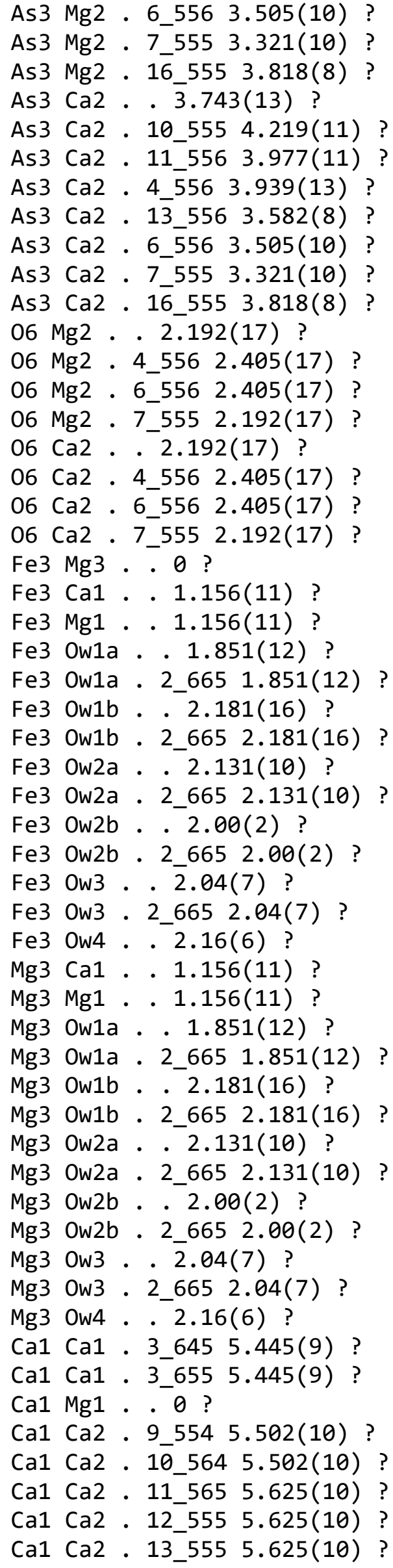




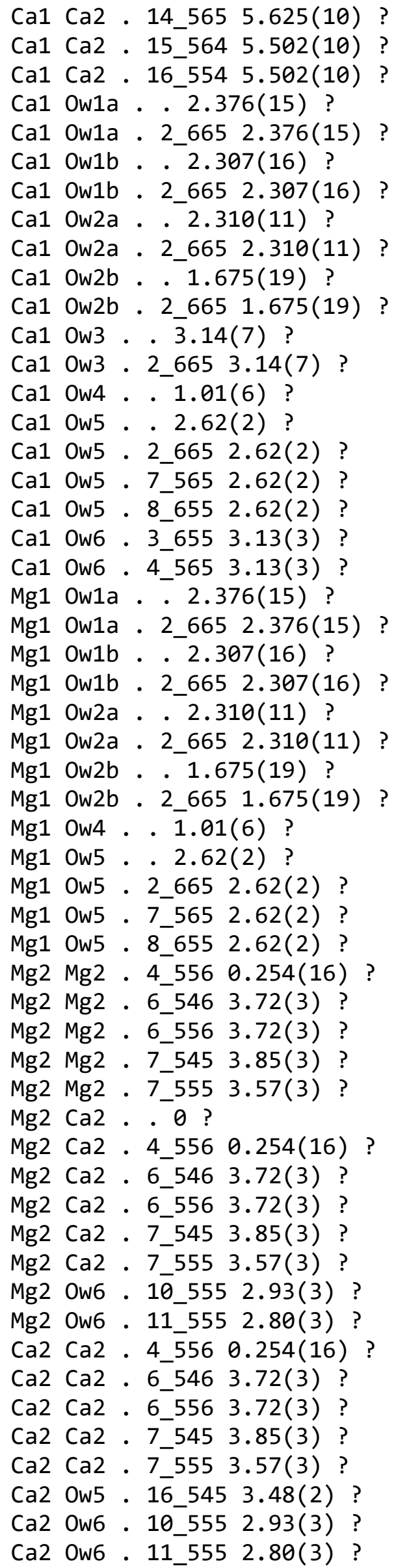




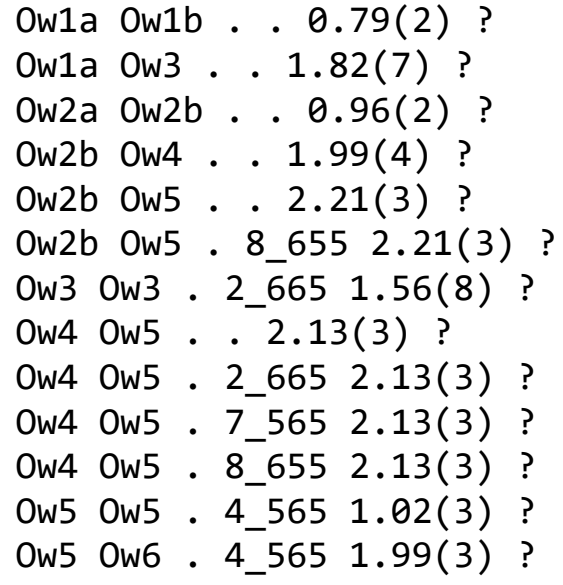




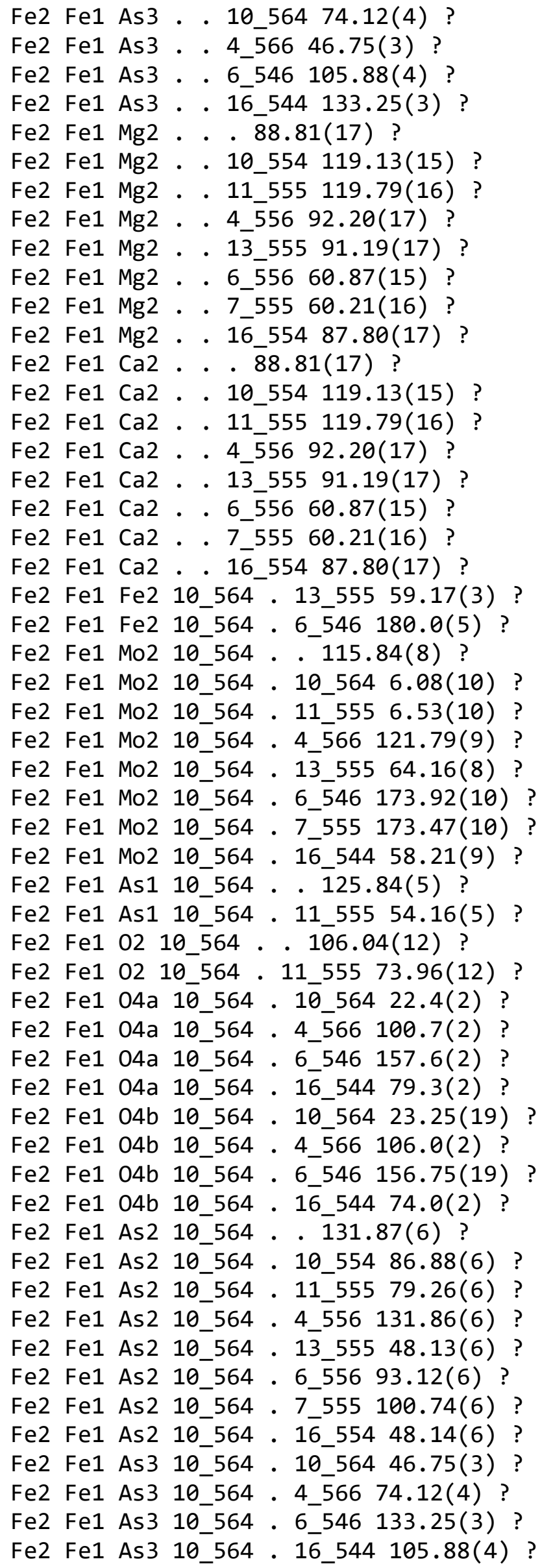


Fe2 Fe1 Mg2 10_564 . . 119.79(16) ?

Fe2 Fe1 Mg2 10_564 . 10_554 92.20(17) ?

Fe2 Fe1 Mg2 10_564 . 11_555 88.81(17) ?

Fe2 Fe1 Mg2 10_564 . 4_556 119.13(15) ?

Fe2 Fe1 Mg2 10_564 . 13_555 60.21(16) ?

Fe2 Fe1 Mg2 10_564 . 6_556 87.80(17) ?

Fe2 Fe1 Mg2 10_564 . 7_555 91.19(17) ?

Fe2 Fe1 Mg2 10_564 . 16_554 60.87(15) ?

Fe2 Fe1 Ca2 10_564 . . 119.79(16) ?

Fe2 Fe1 Ca2 10_564 . 10_554 92.20(17) ?

Fe2 Fe1 Ca2 10_564 . 11_555 88.81(17) ?

Fe2 Fe1 Ca2 10_564 . 4_556 119.13(15) ?

Fe2 Fe1 Ca2 10_564 . 13_555 60.21(16) ?

Fe2 Fe1 Ca2 10_564 . 6_556 87.80(17) ?

Fe2 Fe1 Ca2 10_564 . 7_555 91.19(17) ?

Fe2 Fe1 Ca2 10_564 . 16_554 60.87(15) ?

Fe2 Fe1 Fe2 13_555 . 6_546 120.83(3) ?

Fe2 Fe1 Mo2 13_555 . . 173.47(10) ?

Fe2 Fe1 Mo2 13_555 . 10_564 58.21(9) ?

Fe2 Fe1 Mo2 13_555 . 11_555 64.16(8) ?

Fe2 Fe1 Mo2 13_555 . 4_566 173.92(10) ?

Fe2 Fe1 Mo2 13_555 . 13_555 6.53(10) ?

Fe2 Fe1 Mo2 13_555 . 6_546 121.79(9) ?

Fe2 Fe1 Mo2 13_555 . 7_555 115.84(8) ?

Fe2 Fe1 Mo2 13_555 . 16_544 6.08(10) ?

Fe2 Fe1 As1 13_555 . . 125.84(5) ?

Fe2 Fe1 As1 13_555 . 11_555 54.16(5) ?

Fe2 Fe1 02 13_555 . . 106.04(12) ?

Fe2 Fe1 02 13_555 . 11_555 73.96(12) ?

Fe2 Fe1 04a 13_555 . 10_564 79.3(2)?

Fe2 Fe1 04a 13_555 . 4_566 157.6(2) ?

Fe2 Fe1 04a 13_555 . 6_546 100.7(2) ?

Fe2 Fe1 04a 13_555 . 16_544 22.4(2) ?

Fe2 Fe1 04b 13_555 . 10_564 74.0(2)?

Fe2 Fe1 04b 13_555 . 4_566 156.75(19) ?

Fe2 Fe1 04b 13_555 . 6_546 106.0(2) ?

Fe2 Fe1 04b 13_555 . 16_544 23.25(19) ?

Fe2 Fe1 As2 13_555 . . 100.74(6) ?

Fe2 Fe1 As2 13_555 . 10_554 48.14(6) ?

Fe2 Fe1 As2 13_555 . 11_555 48.13(6) ?

Fe2 Fe1 As2 13_555 . 4_556 93.12(6) ?

Fe2 Fe1 As2 13_555 . 13_555 79.26(6) ?

Fe2 Fe1 As2 13_555 . 6_556 131.86(6) ?

Fe2 Fe1 As2 13_555 . 7_555 131.87(6) ?

Fe2 Fe1 As2 13_555 . 16_554 86.88(6) ?

Fe2 Fe1 As3 13_555 . 10_564 105.88(4) ?

Fe2 Fe1 As3 13_555 . 4_566 133.25(3) ?

Fe2 Fe1 As3 13_555 . 6_546 74.12(4) ?

Fe2 Fe1 As3 13_555 . 16_544 46.75(3) ?

Fe2 Fe1 Mg2 13_555 . . 91.19(17) ?

Fe2 Fe1 Mg2 13_555 . 10_554 60.87(15) ?

Fe2 Fe1 Mg2 13_555 . 11_555 60.21(16) ?

Fe2 Fe1 Mg2 13_555 . 4_556 87.80(17) ?

Fe2 Fe1 Mg2 13_555 . 13_555 88.81(17) ? 
Fe2 Fe1 Mg2 13_555 . 6_556 119.13(15) ?

Fe2 Fe1 Mg2 13_555 . 7_555 119.79(16) ?

Fe2 Fe1 Mg2 13_555 . 16_554 92.20(17) ?

Fe2 Fe1 Ca2 13_555 . . 91.19(17) ?

Fe2 Fe1 Ca2 13_555 . 10_554 60.87(15) ?

Fe2 Fe1 Ca2 13_555 . 11_555 60.21(16) ?

Fe2 Fe1 Ca2 13_555 . 4_556 87.80(17) ?

Fe2 Fe1 Ca2 13_555 . 13_555 88.81(17) ?

Fe2 Fe1 Ca2 13_555 . 6_556 119.13(15) ?

Fe2 Fe1 Ca2 13_555 . 7_555 119.79(16) ?

Fe2 Fe1 Ca2 13_555 . 16_554 92.20(17) ?

Fe2 Fe1 Mo2 6_546 . . 64.16(8) ?

Fe2 Fe1 Mo2 6_546 . 10_564 173.92(10) ?

Fe2 Fe1 Mo2 6_546 . 11_555 173.47(10) ?

Fe2 Fe1 Mo2 6_546 . 4_566 58.21(9) ?

Fe2 Fe1 Mo2 6_546 . 13_555 115.84(8) ?

Fe2 Fe1 Mo2 6_546 . 6_546 6.08(10) ?

Fe2 Fe1 Mo2 6_546 . 7_555 6.53(10) ?

Fe2 Fe1 Mo2 6_546 . 16_544 121.79(9) ?

Fe2 Fe1 As1 6_546 . . 54.16(5) ?

Fe2 Fe1 As1 6_546 . 11_555 125.84(5) ?

Fe2 Fe1 02 6_546 . 73.96(12) ?

Fe2 Fe1 02 6_546 . 11_555 106.04(12) ?

Fe2 Fe1 04a 6_546 . 10_564 157.6(2) ?

Fe2 Fe1 04a 6_546 . 4_566 79.3(2) ?

Fe2 Fe1 04a 6_546 . 6_546 22.4(2) ?

Fe2 Fe1 04a 6_546 . 16_544 100.7(2) ?

Fe2 Fe1 04b 6_546 . 10_564 156.75(19) ?

Fe2 Fe1 04b 6_546 . 4_566 74.0(2) ?

Fe2 Fe1 04b 6_546 . 6_546 23.25(19) ?

Fe2 Fe1 04b 6_546 . 16_544 106.0(2) ?

Fe2 Fe1 As2 6_546 . . 48.13(6) ?

Fe2 Fe1 As2 6_546 . 10_554 93.12(6) ?

Fe2 Fe1 As2 6_546. 11_555 100.74(6) ?

Fe2 Fe1 As2 6_546 . 4_556 48.14(6) ?

Fe2 Fe1 As2 6_546 . 13_555 131.87(6) ?

Fe2 Fe1 As2 6_546 . 6_556 86.88(6) ?

Fe2 Fe1 As2 6_546 . 7_555 79.26(6) ?

Fe2 Fe1 As2 6_546 . 16_554 131.86(6) ?

Fe2 Fe1 As3 6_546 . 10_564 133.25(3) ?

Fe2 Fe1 As3 6_546 . 4_566 105.88(4) ?

Fe2 Fe1 As3 6_546 . 6_546 46.75(3) ?

Fe2 Fe1 As3 6_546 . 16_544 74.12(4) ?

Fe2 Fe1 Mg2 6_546 . . 60.21(16) ?

Fe2 Fe1 Mg2 6_546 . 10_554 87.80(17) ?

Fe2 Fe1 Mg2 6_546 . 11_555 91.19(17) ?

Fe2 Fe1 Mg2 6_546 . 4_556 60.87(15)?

Fe2 Fe1 Mg2 6_546 . 13_555 119.79(16) ?

Fe2 Fe1 Mg2 6_546 . 6_556 92.20(17) ?

Fe2 Fe1 Mg2 6_546 . 7_555 88.81(17) ?

Fe2 Fe1 Mg2 6_546 . 16_554 119.13(15) ?

Fe2 Fe1 Ca2 6_546 . . 60.21(16) ?

Fe2 Fe1 Ca2 6_546 . 10_554 87.80(17) ?

Fe2 Fe1 Ca2 6_546 . 11_555 91.19(17) ? 
Fe2 Fe1 Ca2 6_546 . 4_556 60.87(15) ?

Fe2 Fe1 Ca2 6_546 . 13_555 119.79(16) ?

Fe2 Fe1 Ca2 6_546 . 6_556 92.20(17) ?

Fe2 Fe1 Ca2 6_546 . 7_555 88.81(17) ?

Fe2 Fe1 Ca2 6_546 . 16_554 119.13(15) ?

Mo2 Fe1 Mo2 . . 10_564 117.33(12) ?

Mo2 Fe1 Mo2 . . 11_555 111.22(12) ?

Mo2 Fe1 Mo2 . . 4_566 6.27(12) ?

Mo2 Fe1 Mo2 . . 13_555 180.0(5) ?

Mo2 Fe1 Mo2 . 6_546 62.67(12) ?

Mo2 Fe1 Mo2 . . 7_555 68.78(12) ?

Mo2 Fe1 Mo2 . . 16_544 173.73(12) ?

Mo2 Fe1 As1 . . 60.15(9) ?

Mo2 Fe1 As1 . . 11_555 119.85(9) ?

Mo2 Fe1 02 . . 79.05(14) ?

Mo2 Fe1 02 . . 11_555 100.95(14) ?

Mo2 Fe1 04a . . 10_564 96.7(2) ?

Mo2 Fe1 04a . . 4_566 16.1(2) ?

Mo2 Fe1 04a . 6_546 83.3(2) ?

Mo2 Fe1 04a . . 16_544 163.9(2) ?

Mo2 Fe1 04b . . 10_564 102.7(2) ?

Mo2 Fe1 04b . . 4_566 17.0(2) ?

Mo2 Fe1 04b . 6_546 77.3(2) ?

Mo2 Fe1 04b . 16_544 163.0(2) ?

Mo2 Fe1 As2 . . 79.49(10) ?

Mo2 Fe1 As2 . . 10_554 137.87(9) ?

Mo2 Fe1 As2 . . 11_555 137.13(9) ?

Mo2 Fe1 As2 . . 4_556 87.69(10) ?

Mo2 Fe1 As2 . . 13_555 100.51(10) ?

Mo2 Fe1 As2 . . 6_556 42.13(9) ?

Mo2 Fe1 As2 . 7 7555 42.87(9) ?

Mo2 Fe1 As2 . . 16_554 92.31(10) ?

Mo2 Fe1 As3 . . 10_564 69.13(9) ?

Mo2 Fe1 As3 . 4 4_566 42.21(9) ?

Mo2 Fe1 As3 . 6_546 110.87(9) ?

Mo2 Fe1 As3 . . 16_544 137.79(9) ?

Mo2 Fe1 Mg2 . . 88.0(2) ?

Mo2 Fe1 Mg2 . . 10_554 124.95(18) ?

Mo2 Fe1 Mg2 . . 11_555 125.36(18) ?

Mo2 Fe1 Mg2 . . 4_556 91.6(2) ?

Mo2 Fe1 Mg2 . . 13_555 92.0(2) ?

Mo2 Fe1 Mg2 . . 6_556 55.05(18) ?

Mo2 Fe1 Mg2 . . 7_555 54.64(18) ?

Mo2 Fe1 Mg2 . . 16_554 88.4(2) ?

Mo2 Fe1 Ca2 . . 88.0(2) ?

Mo2 Fe1 Ca2 . . 10_554 124.95(18) ?

Mo2 Fe1 Ca2 . . 11_555 125.36(18) ?

Mo2 Fe1 Ca2 . . 4_556 91.6(2) ?

Mo2 Fe1 Ca2 . . 13_555 92.0(2) ?

Mo2 Fe1 Ca2 . 6_556 55.05(18) ?

Mo2 Fe1 Ca2 . 7 7555 54.64(18)?

Mo2 Fe1 Ca2 . . 16_554 88.4(2) ?

Mo2 Fe1 Mo2 10_564 - 11_555 6.27(12) ?

Mo2 Fe1 Mo2 10_564 . 4_566 123.47(12) ? 


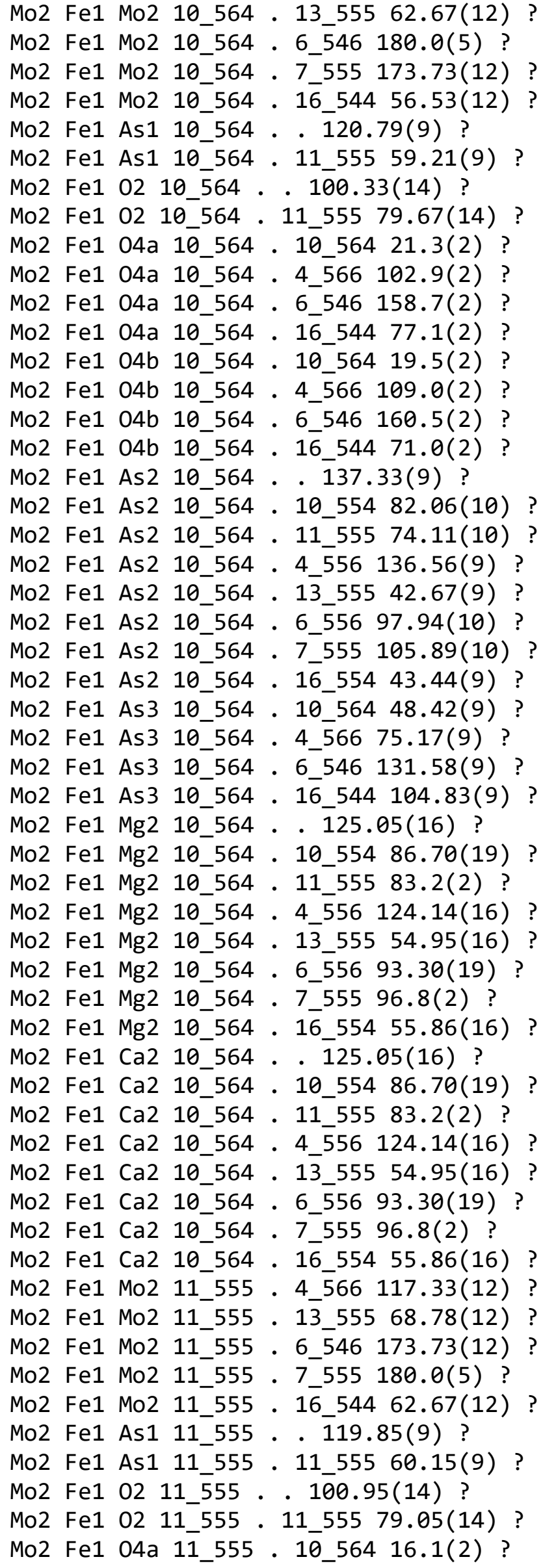




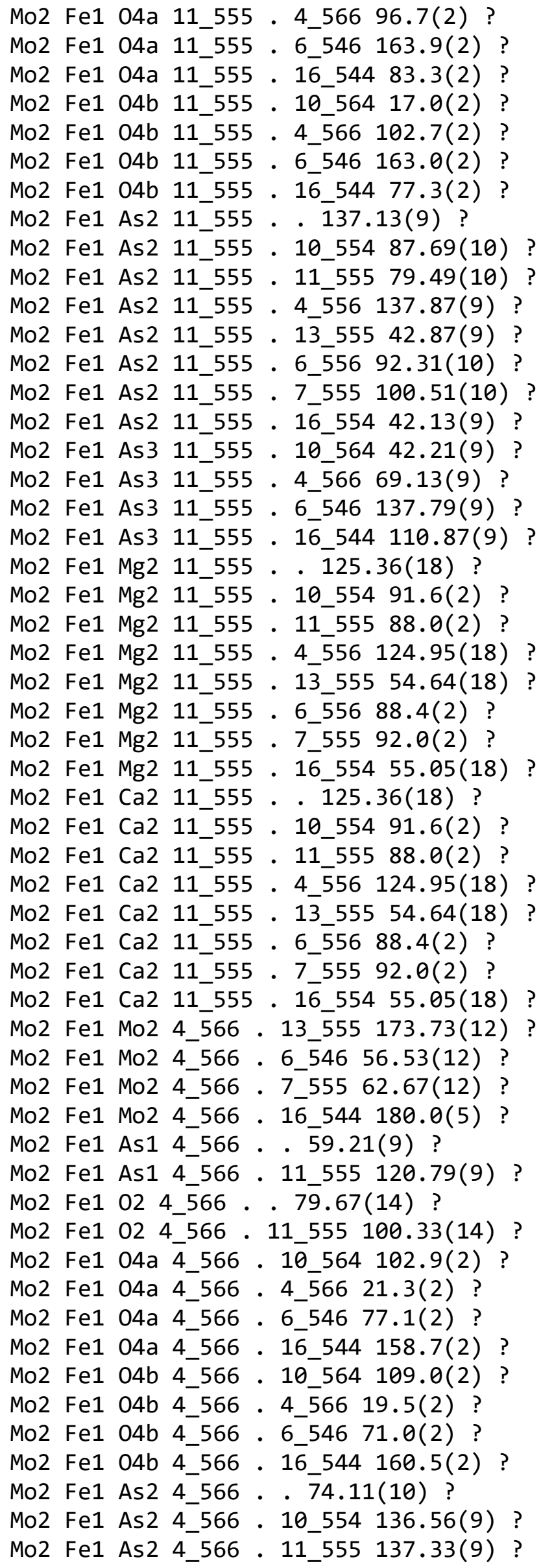




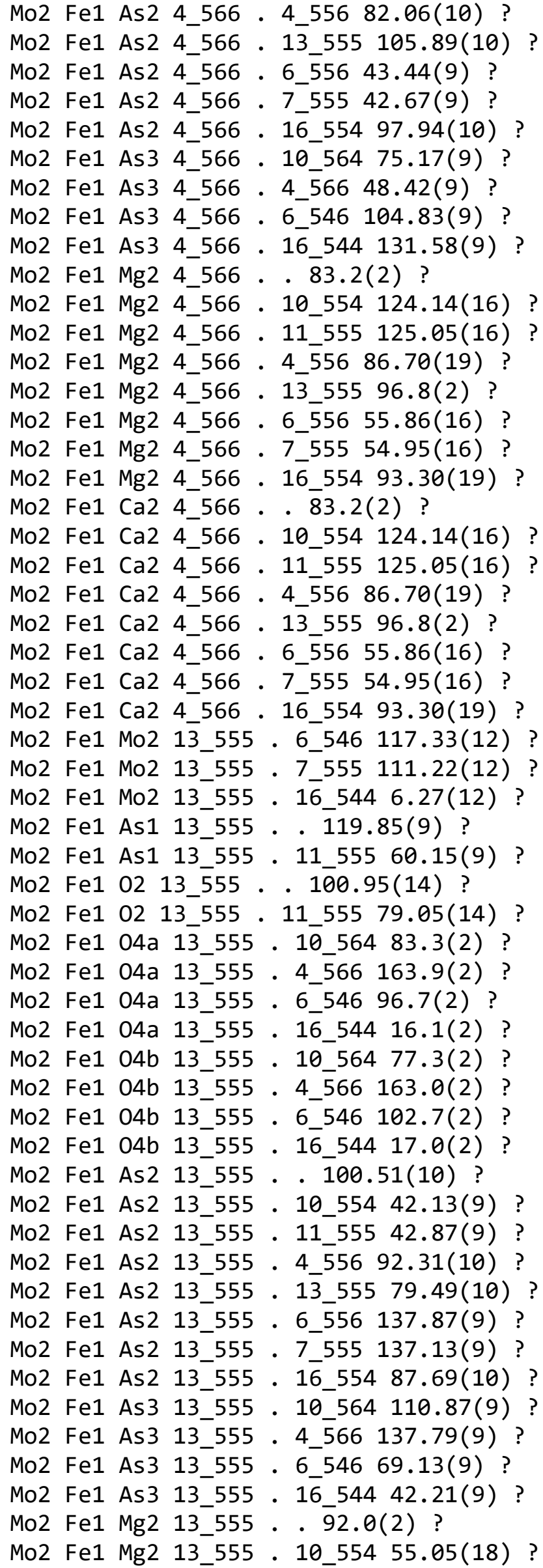




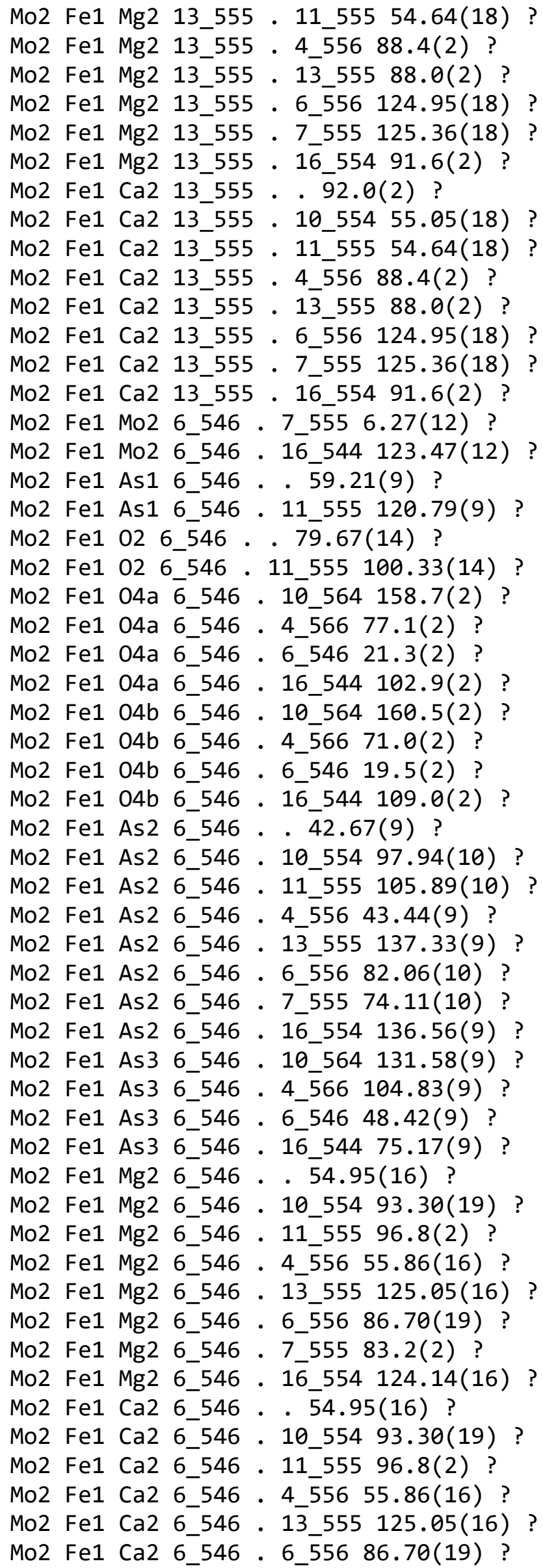




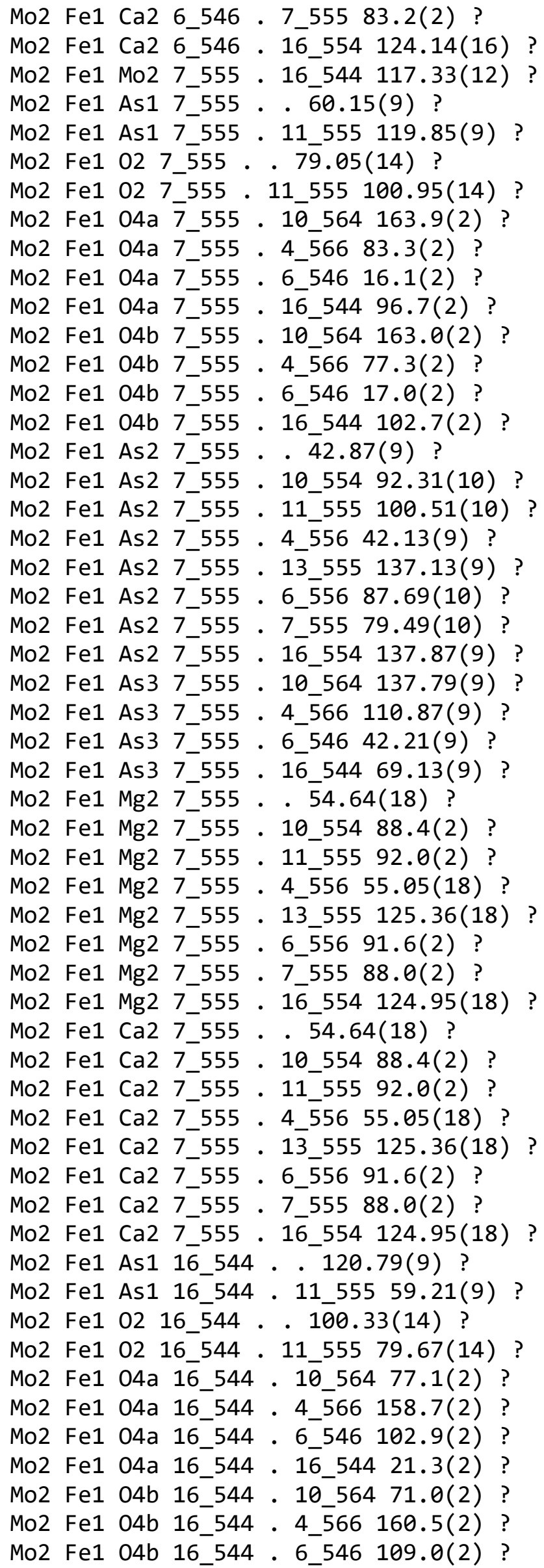




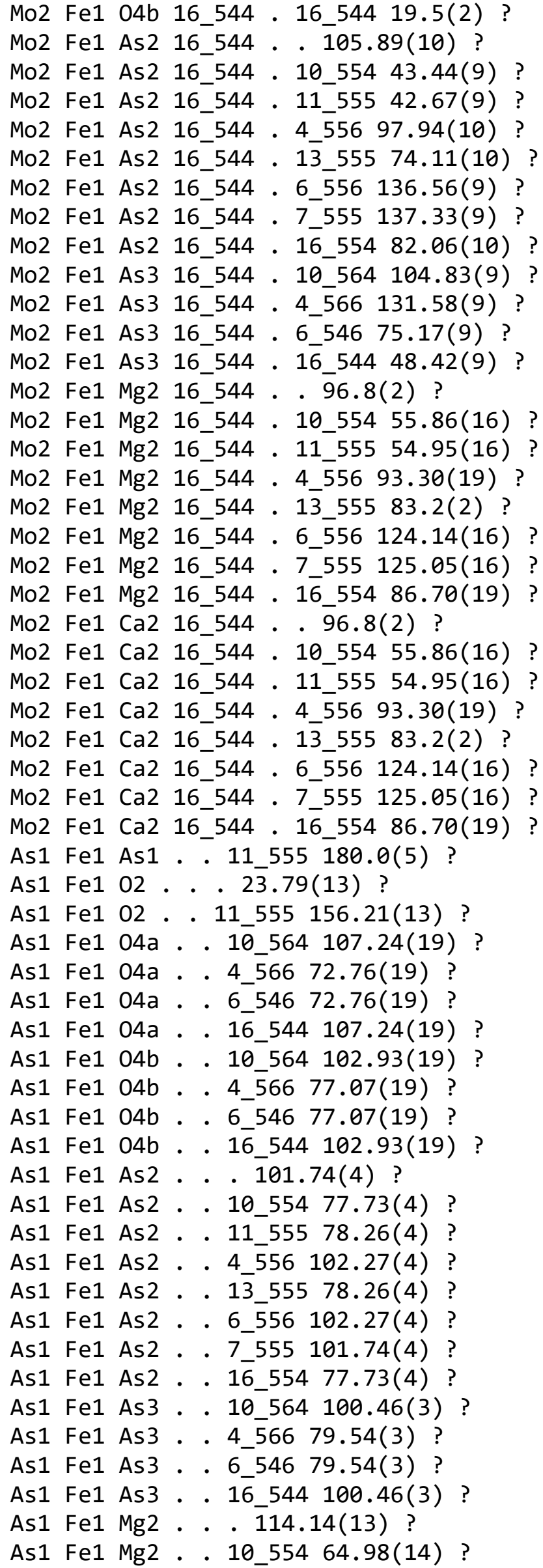




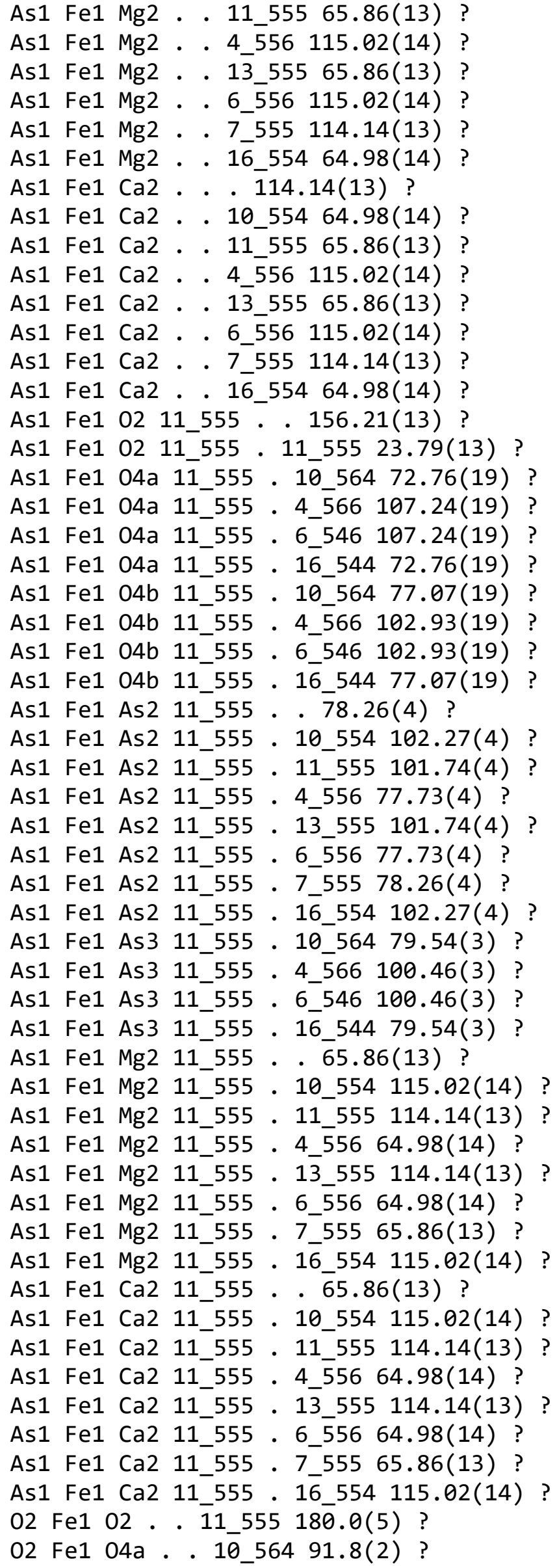




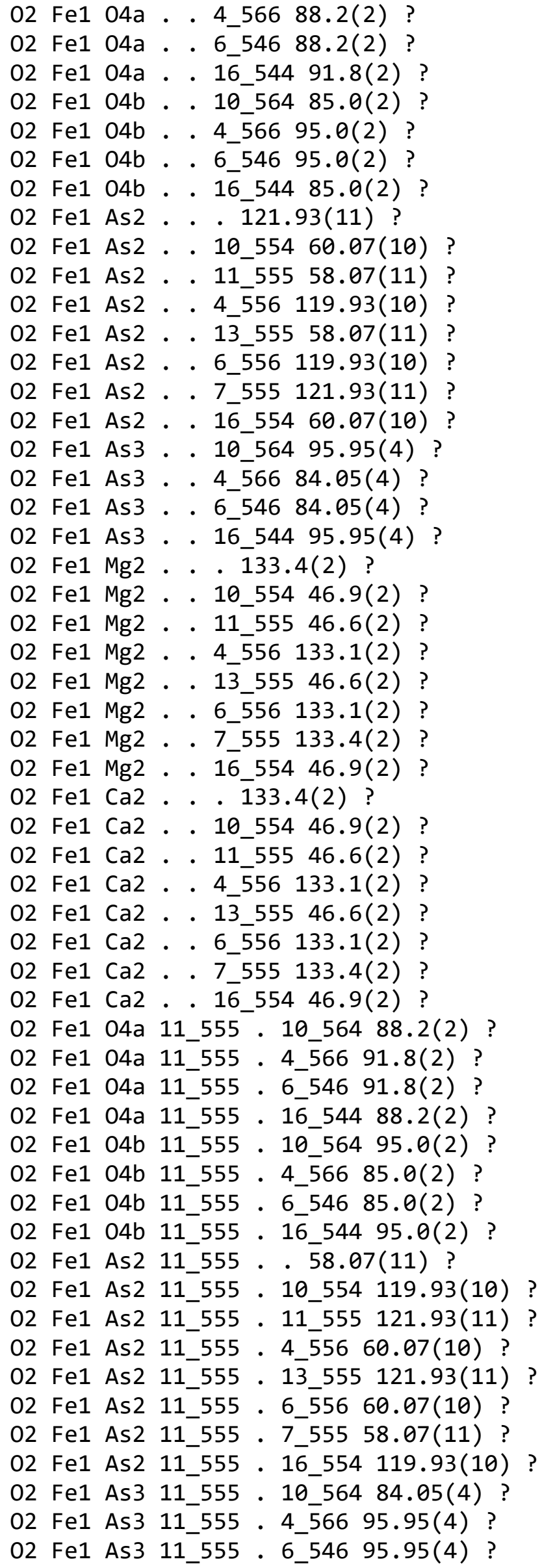




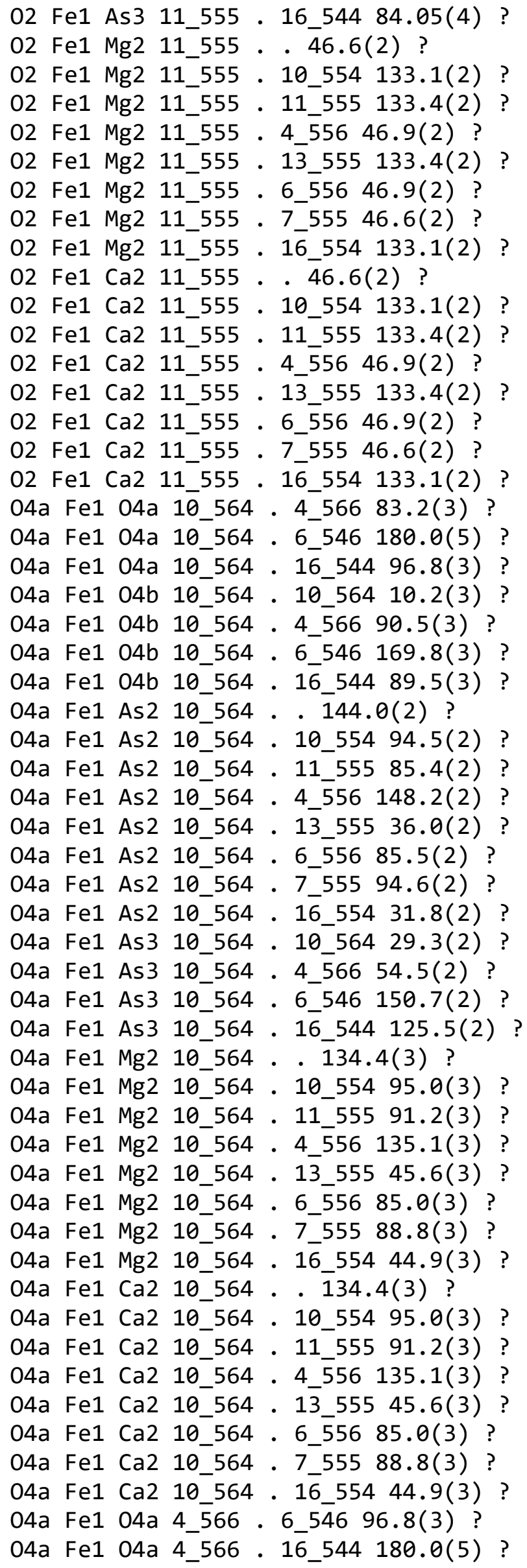




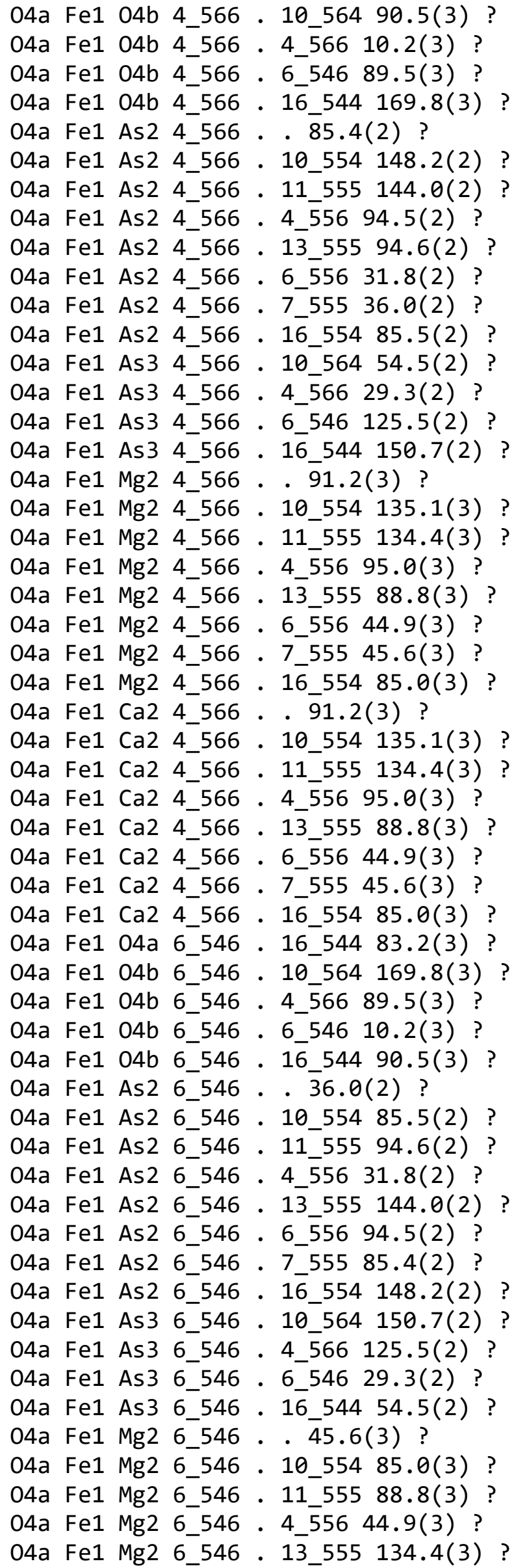




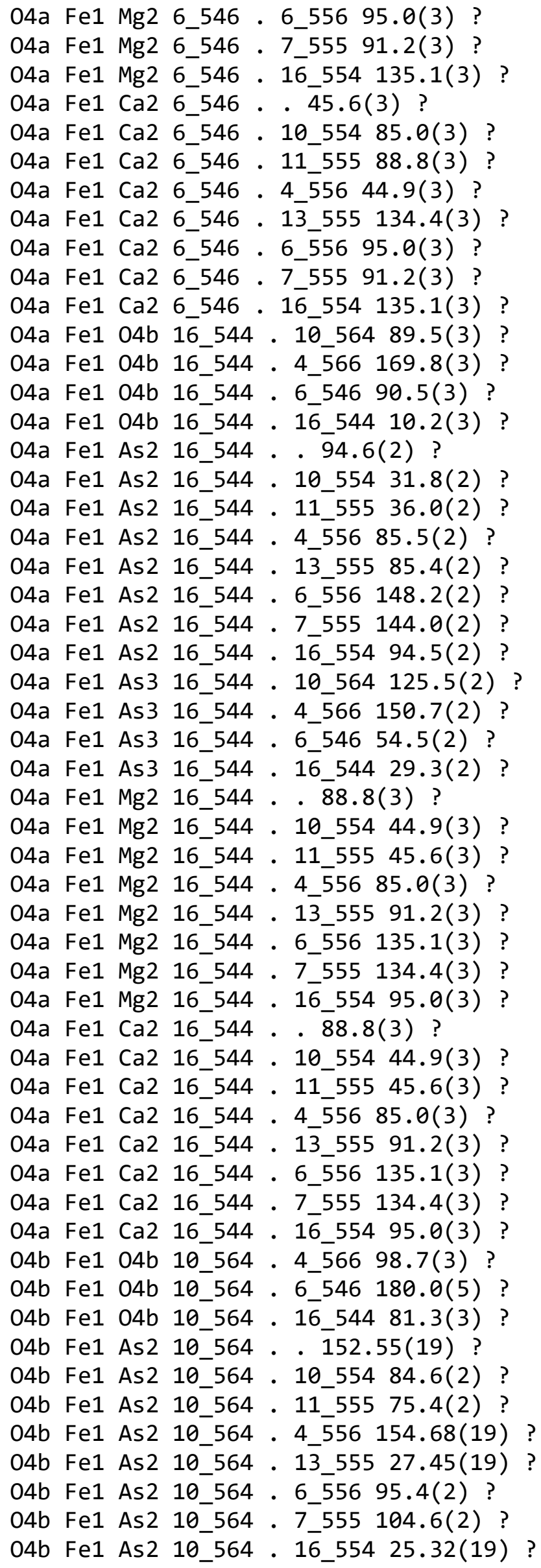




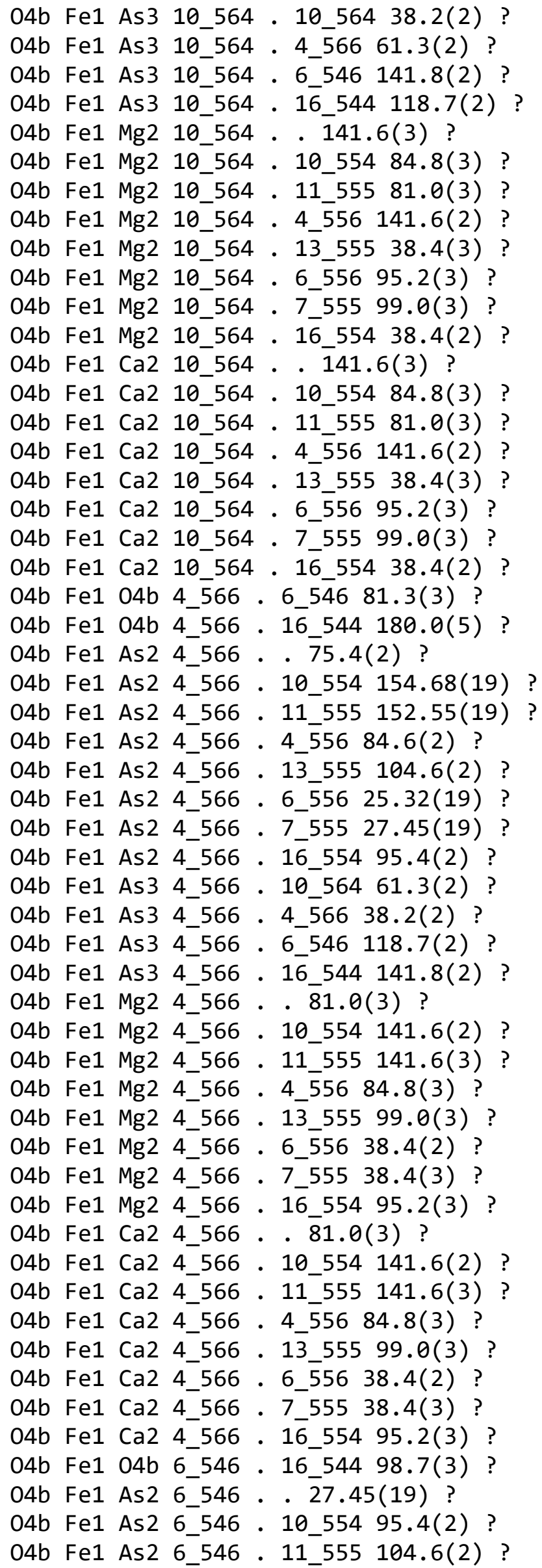




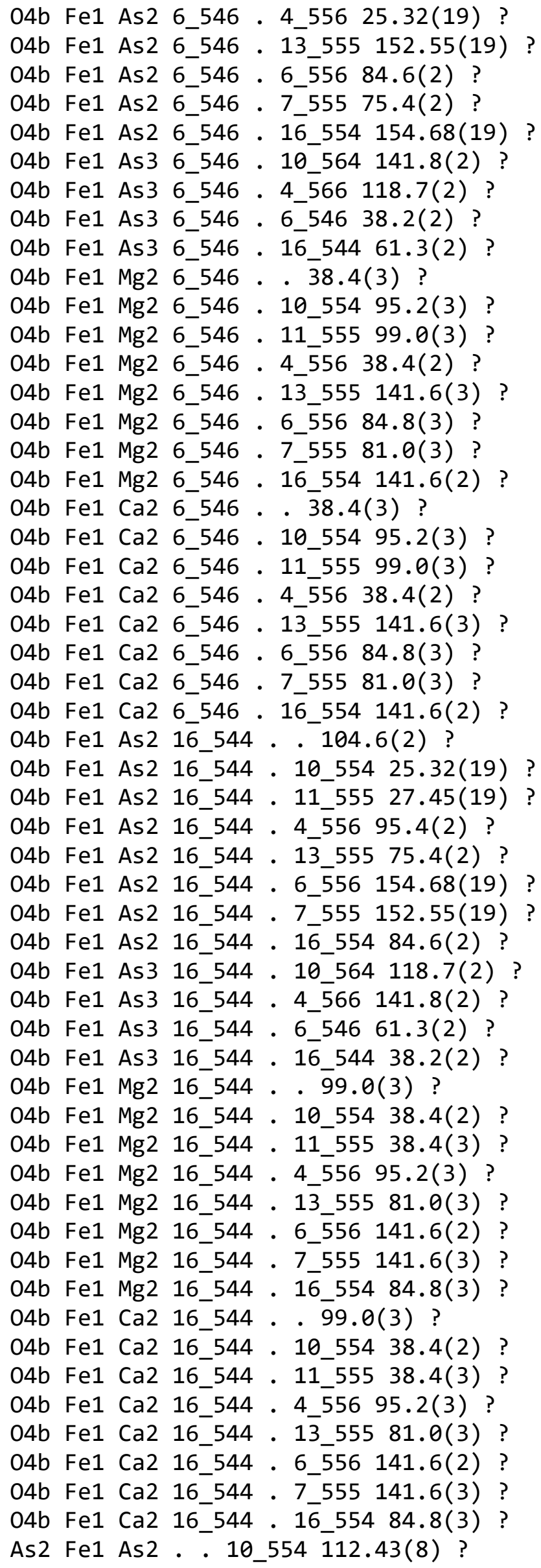




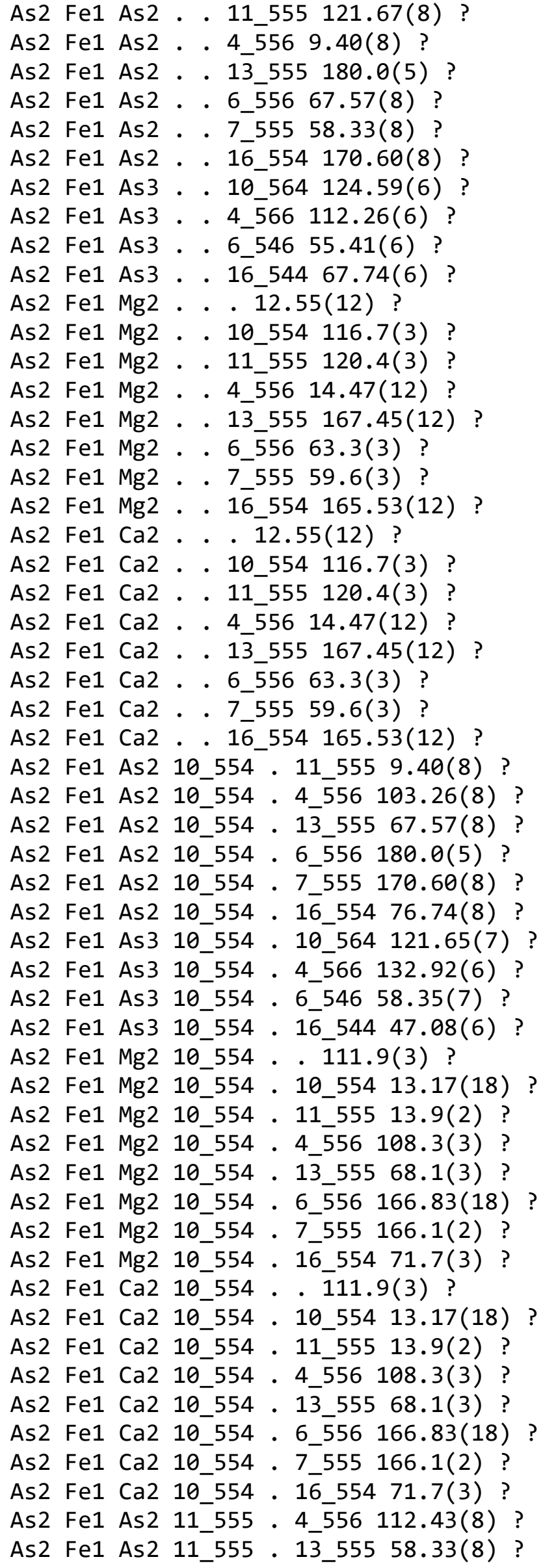




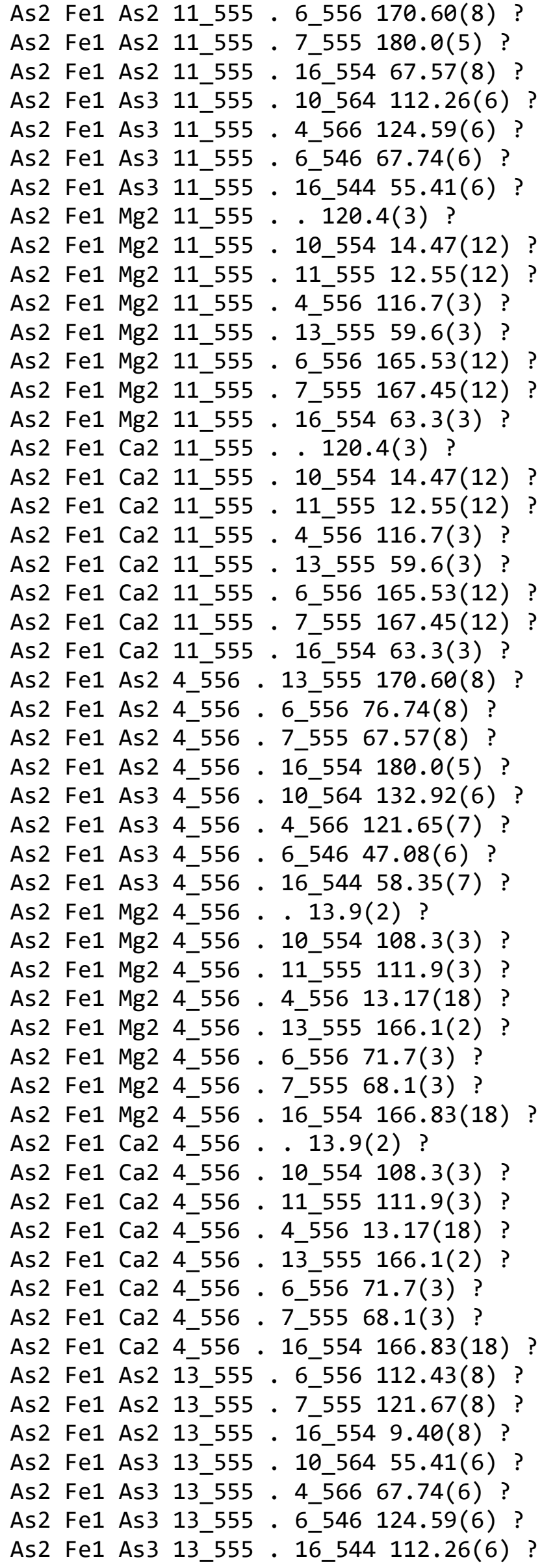




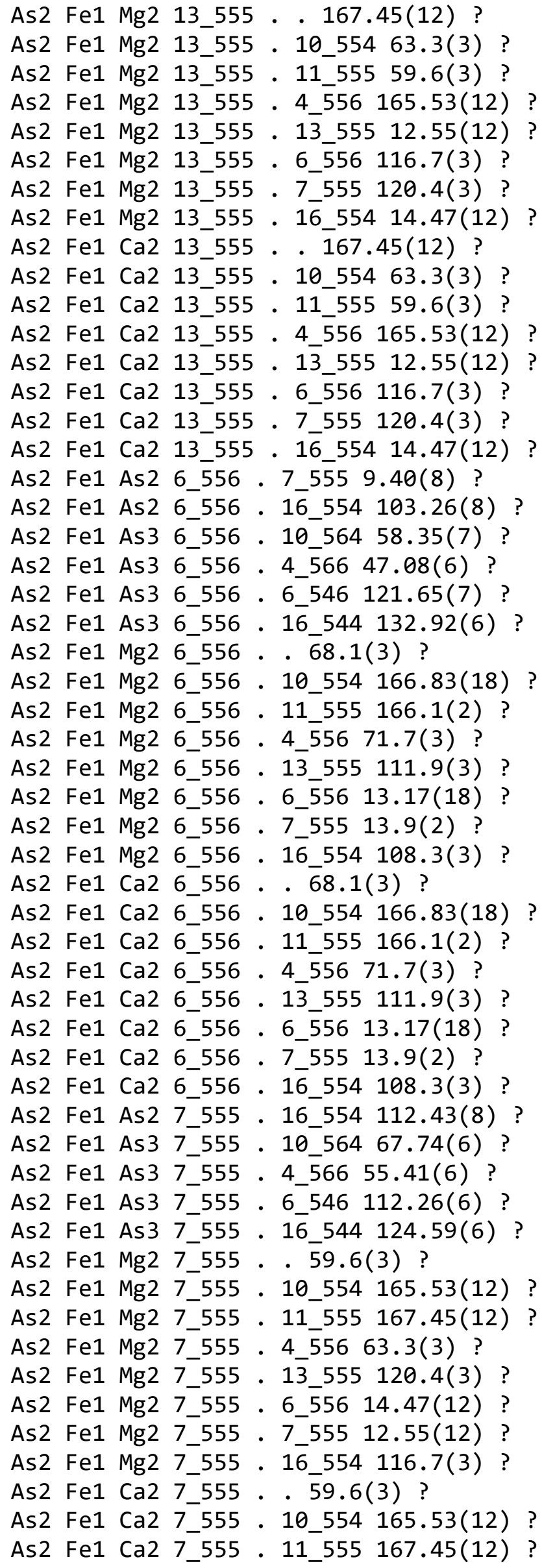




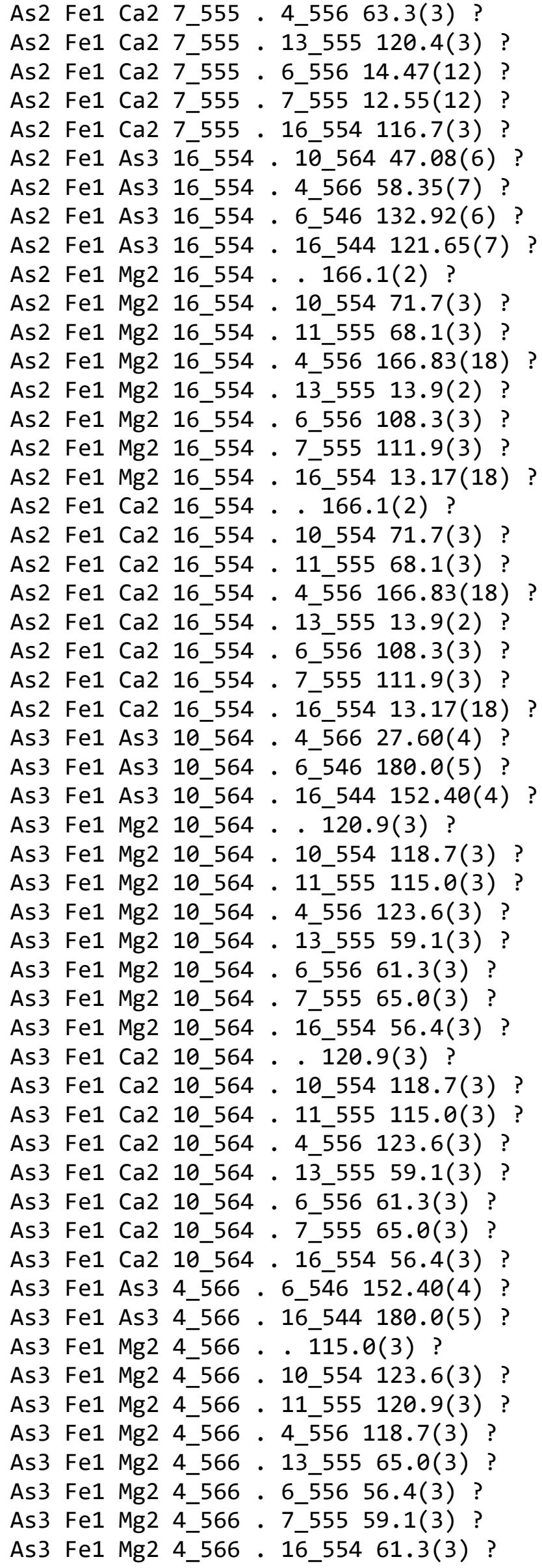




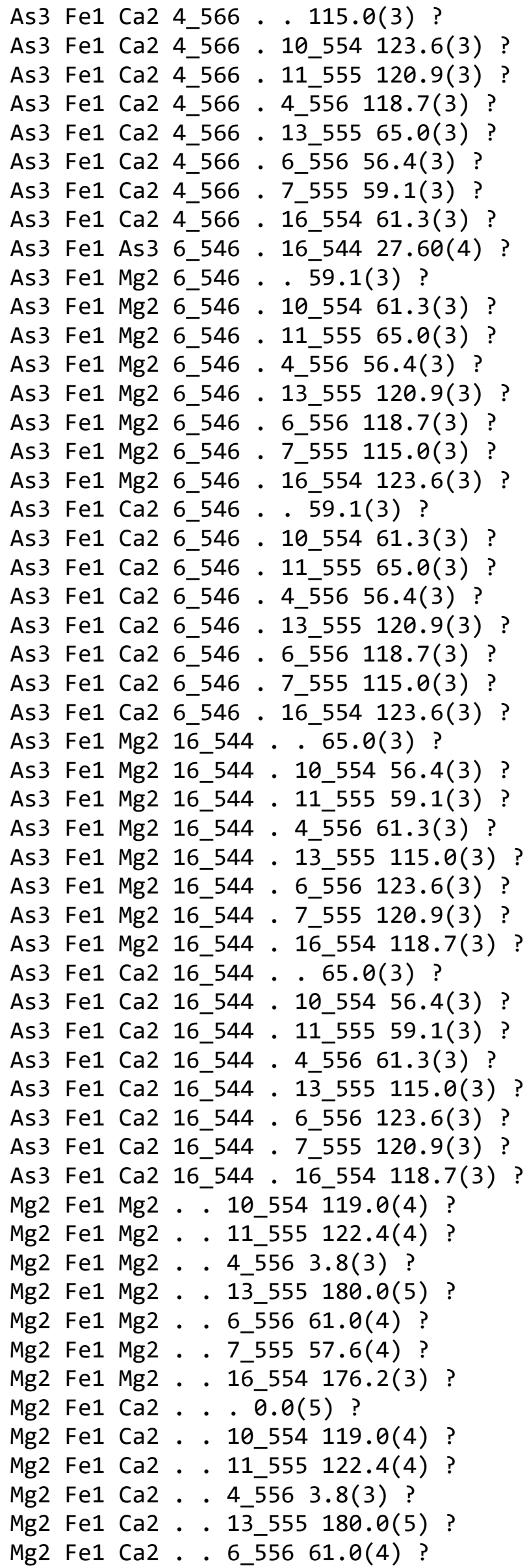




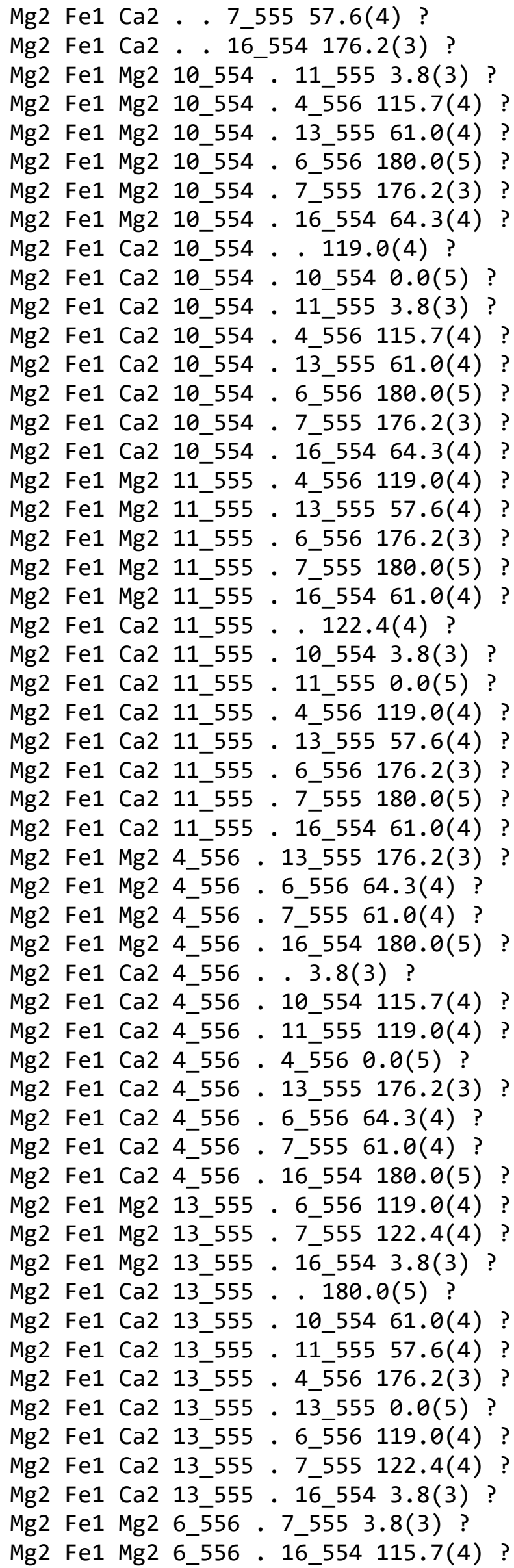




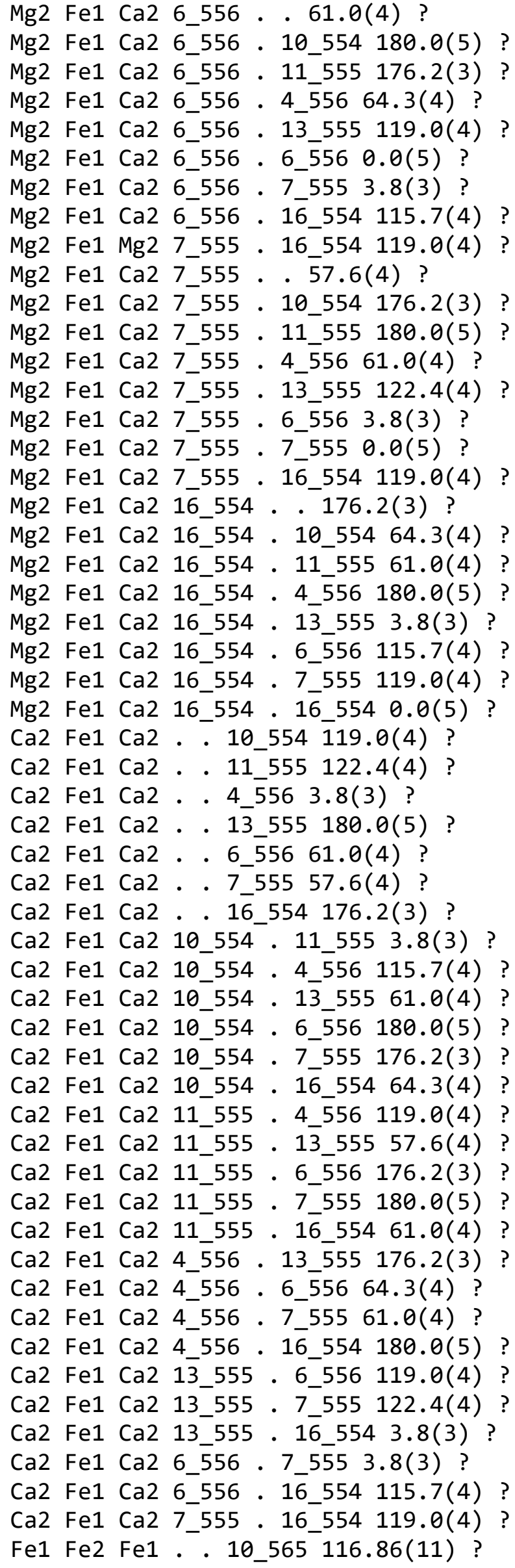




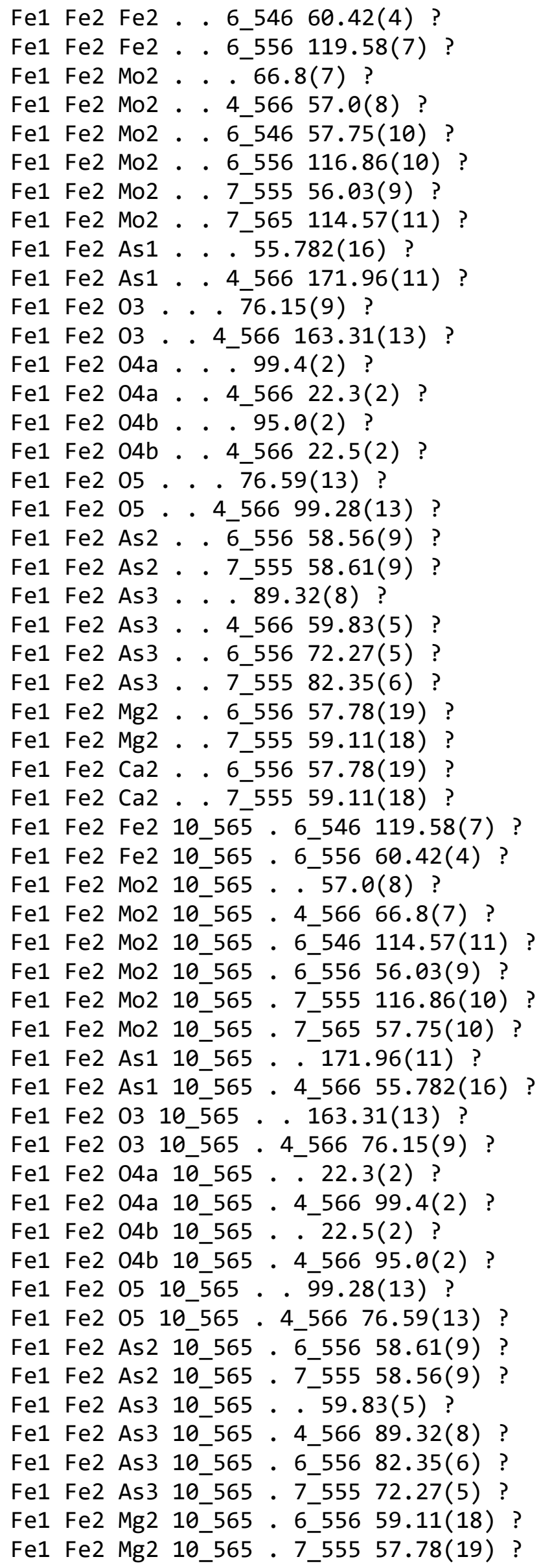




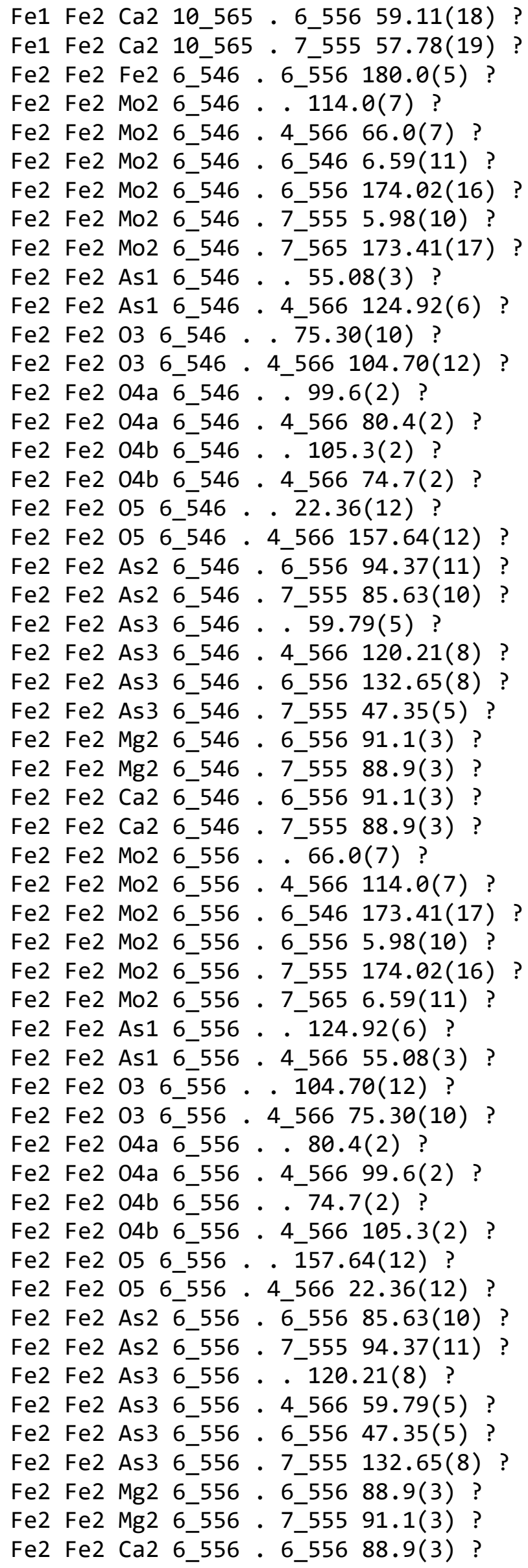




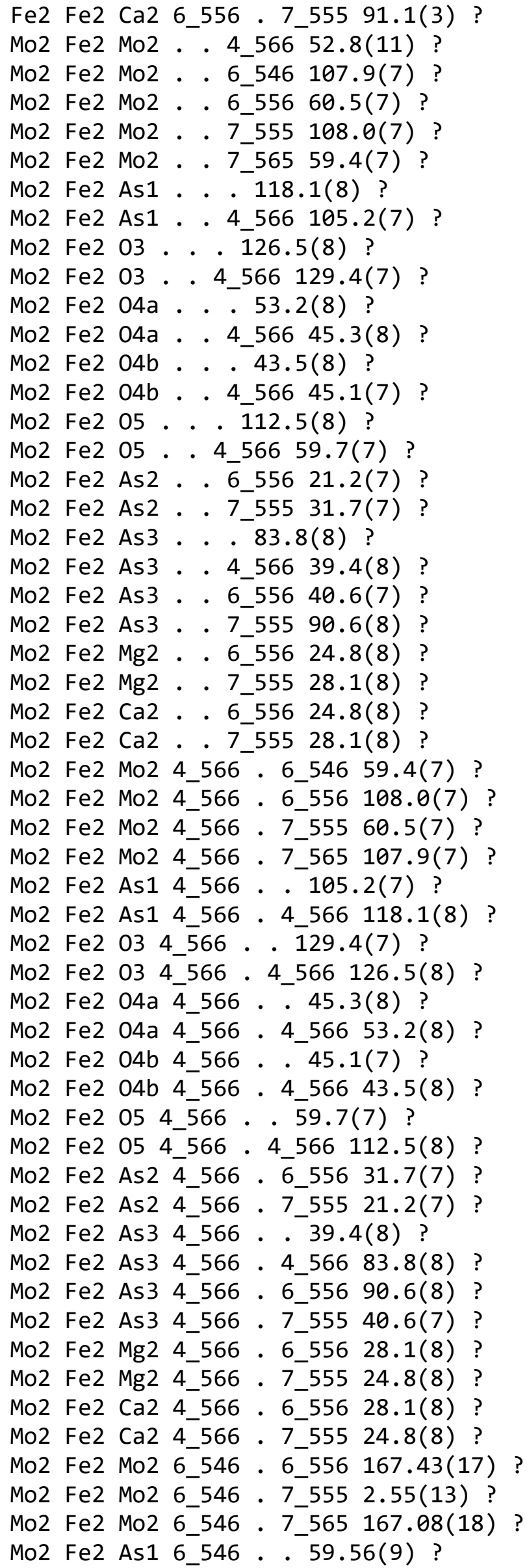




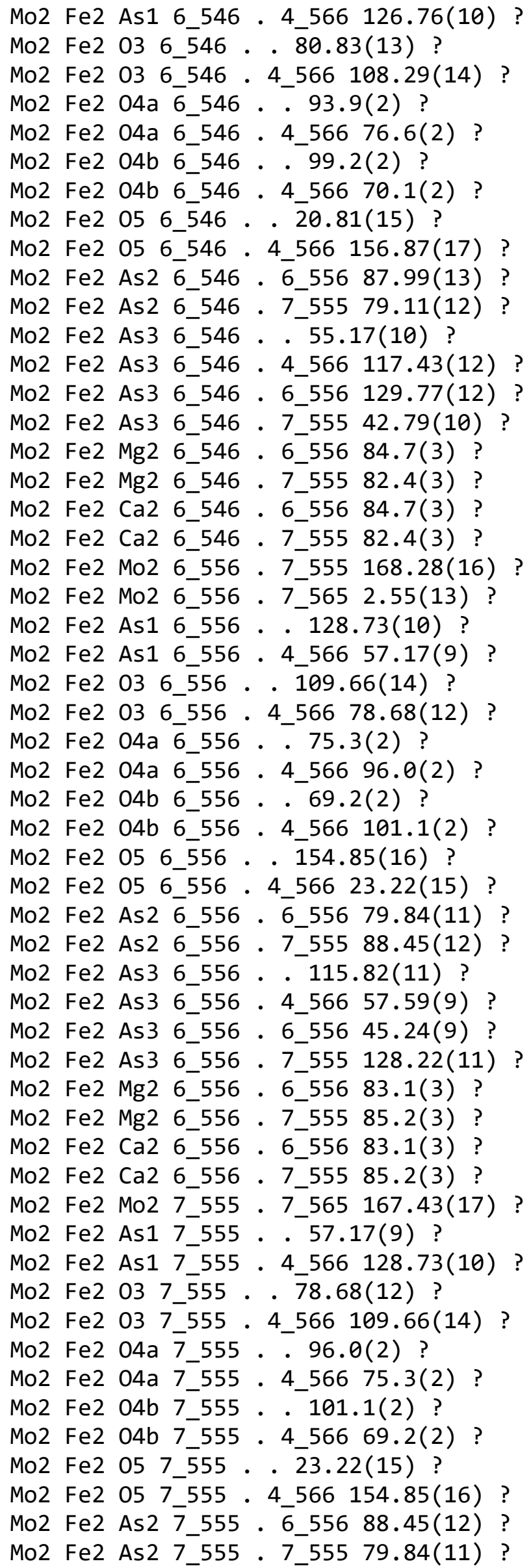




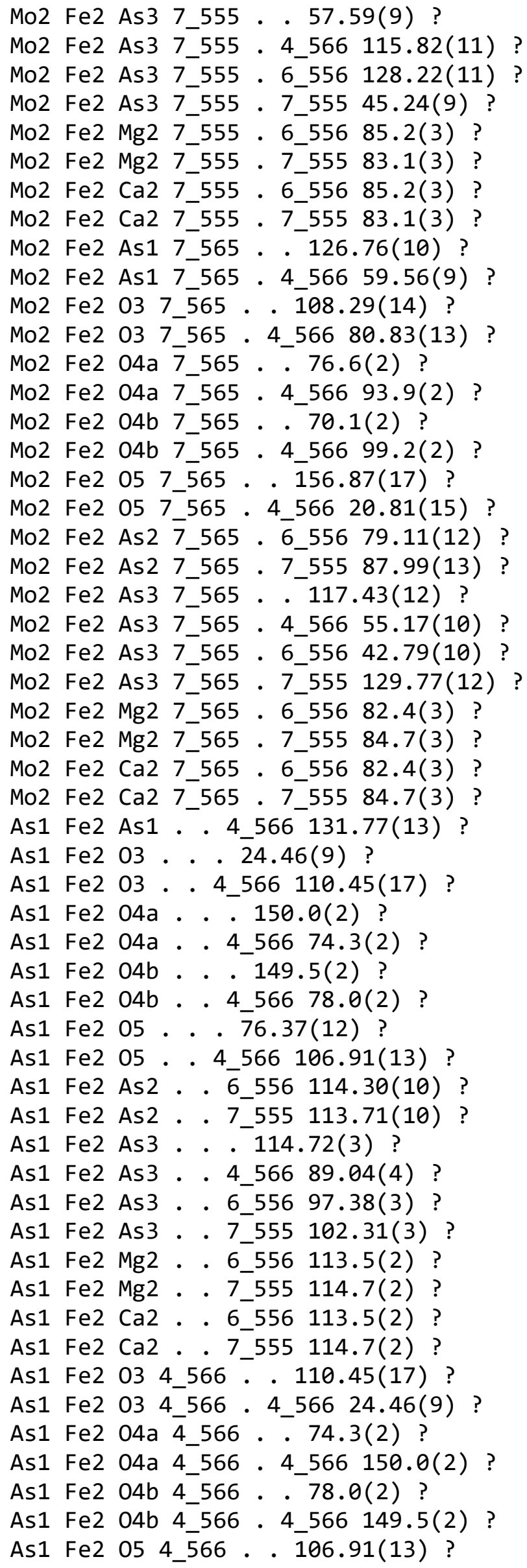




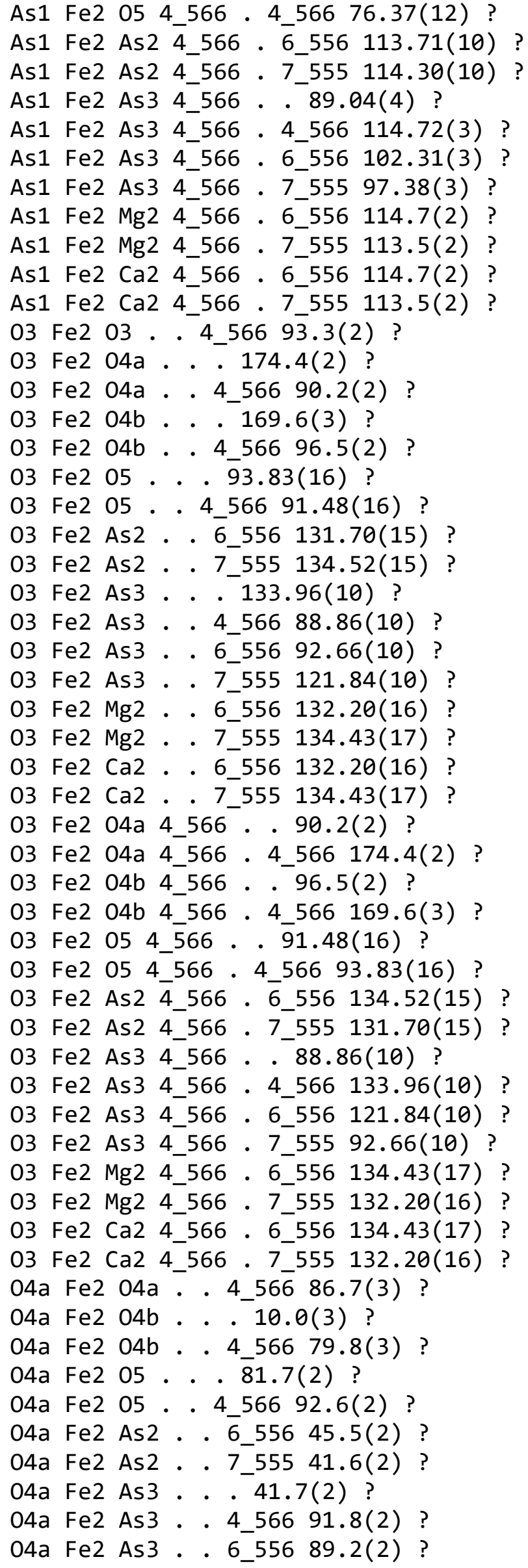




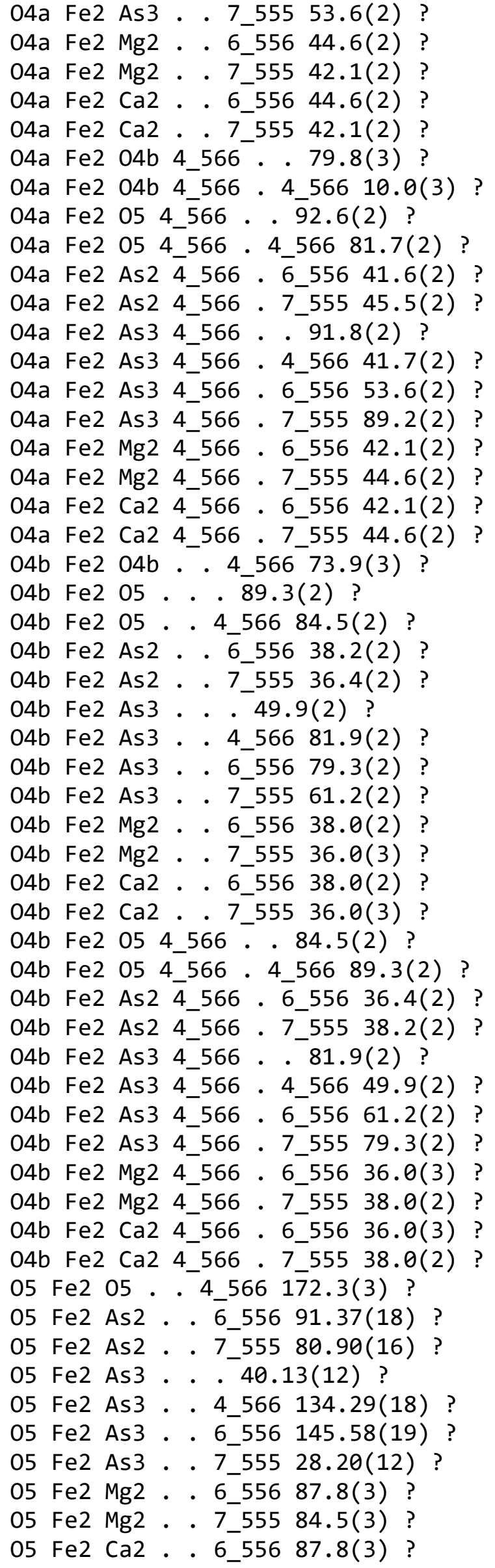




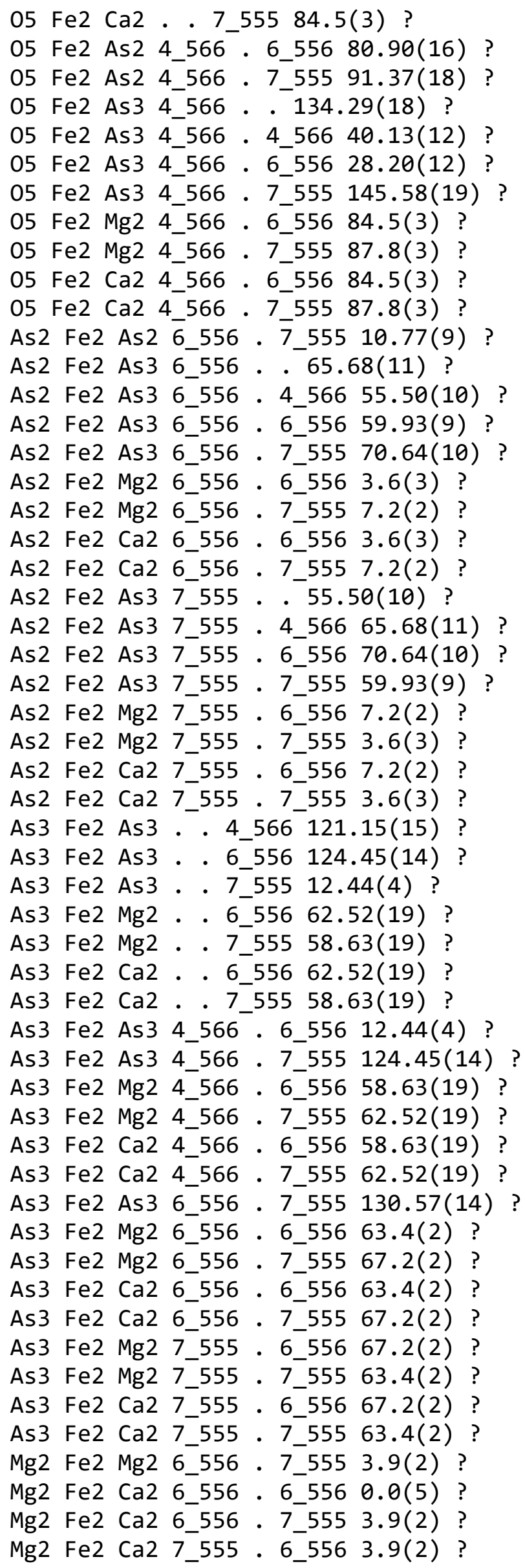




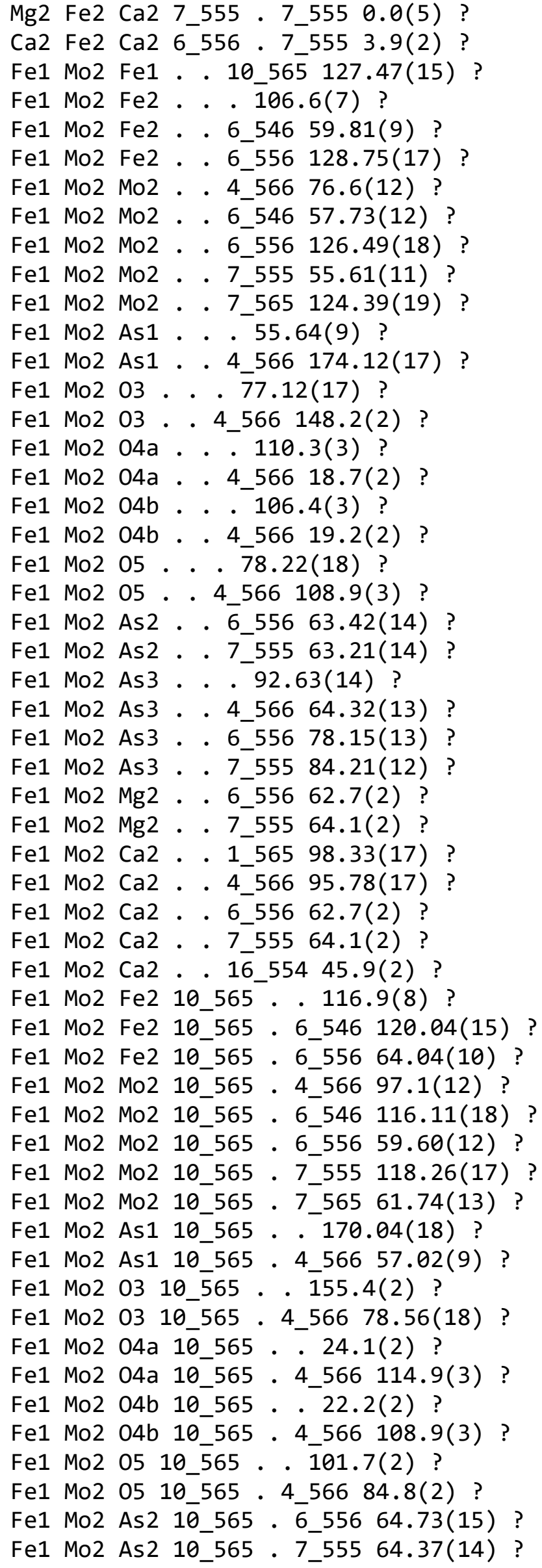




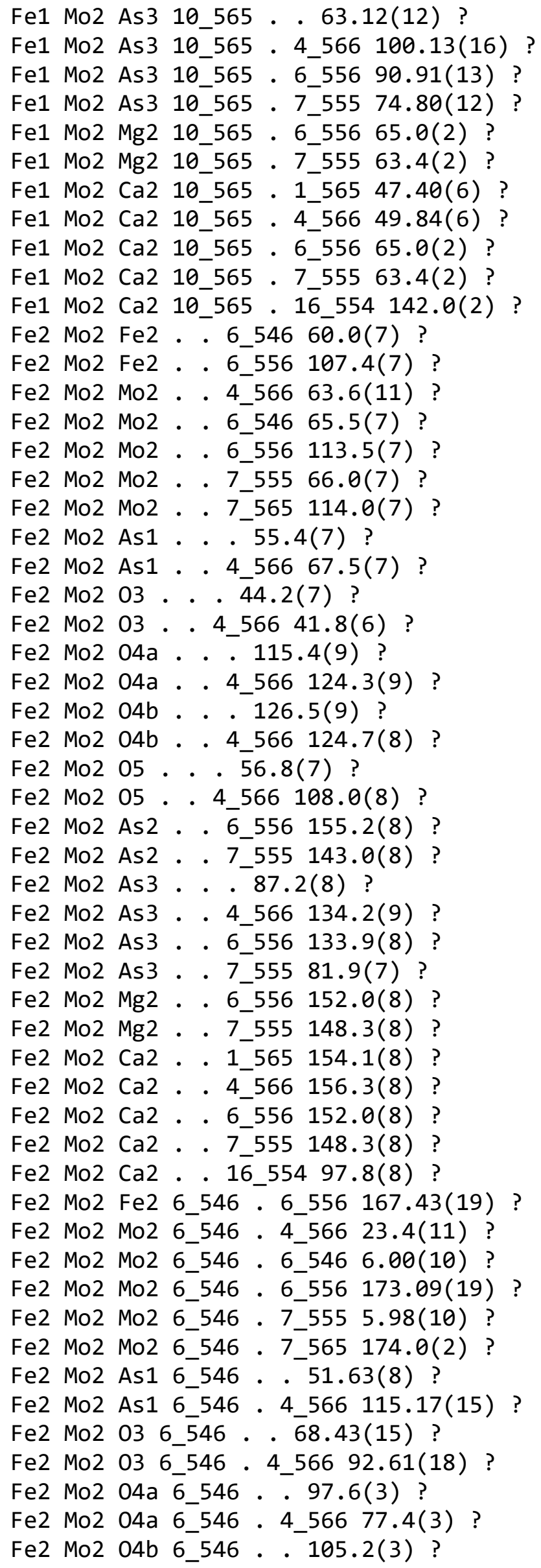




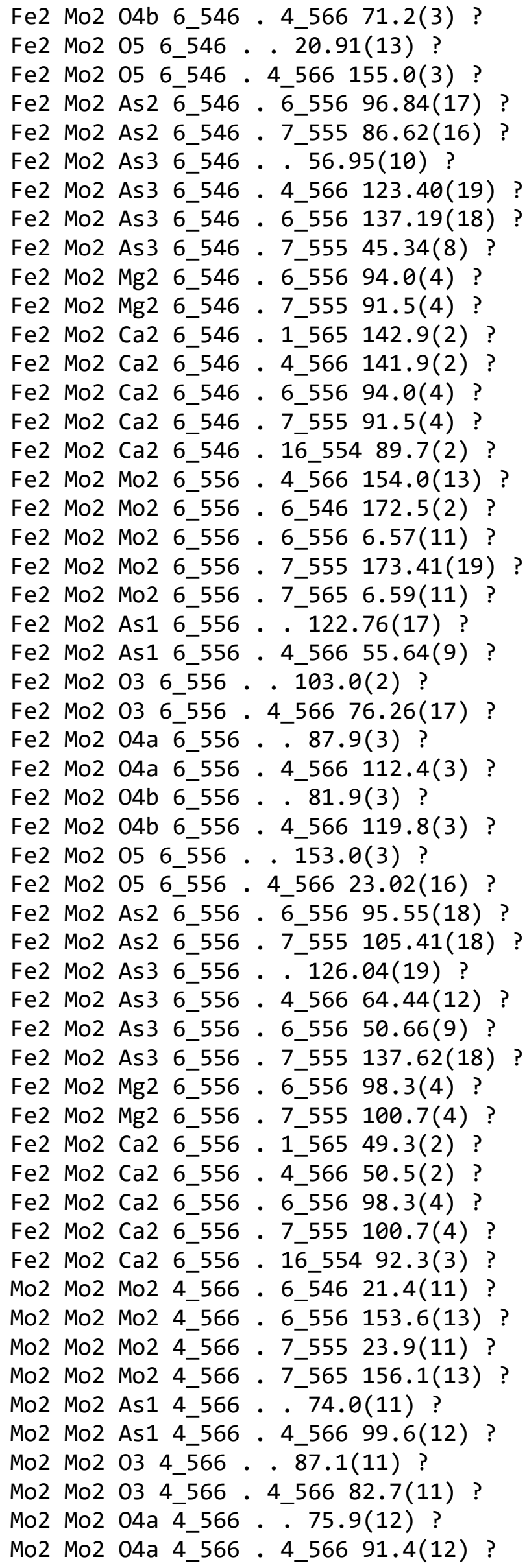




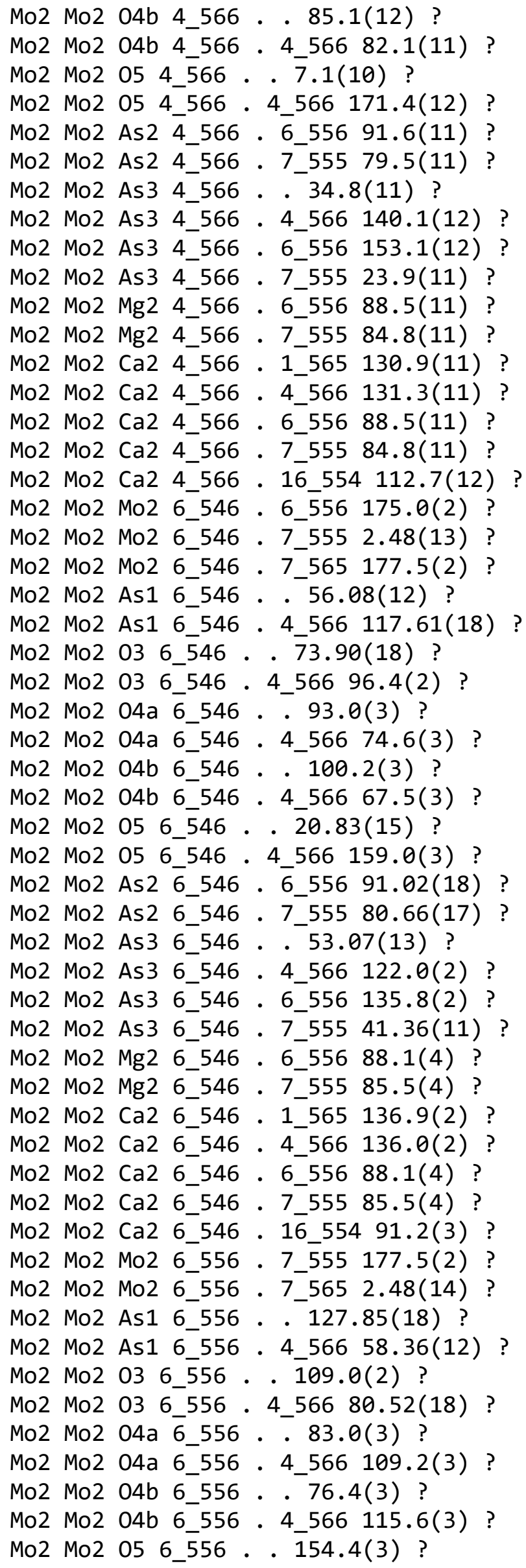




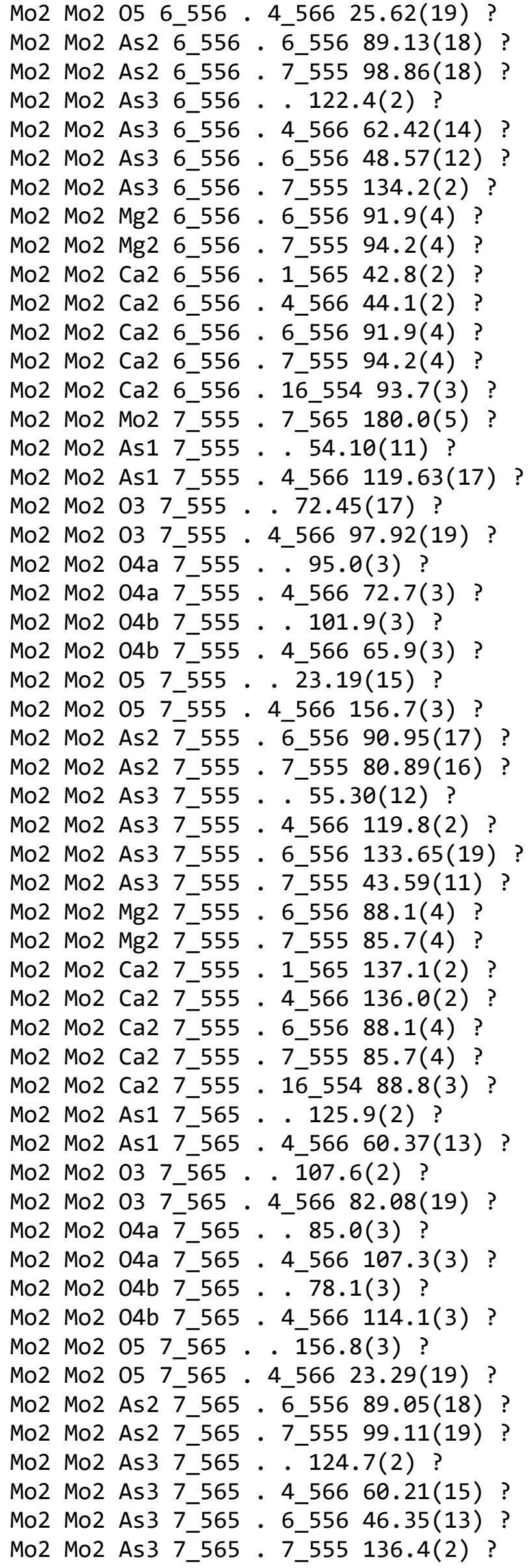




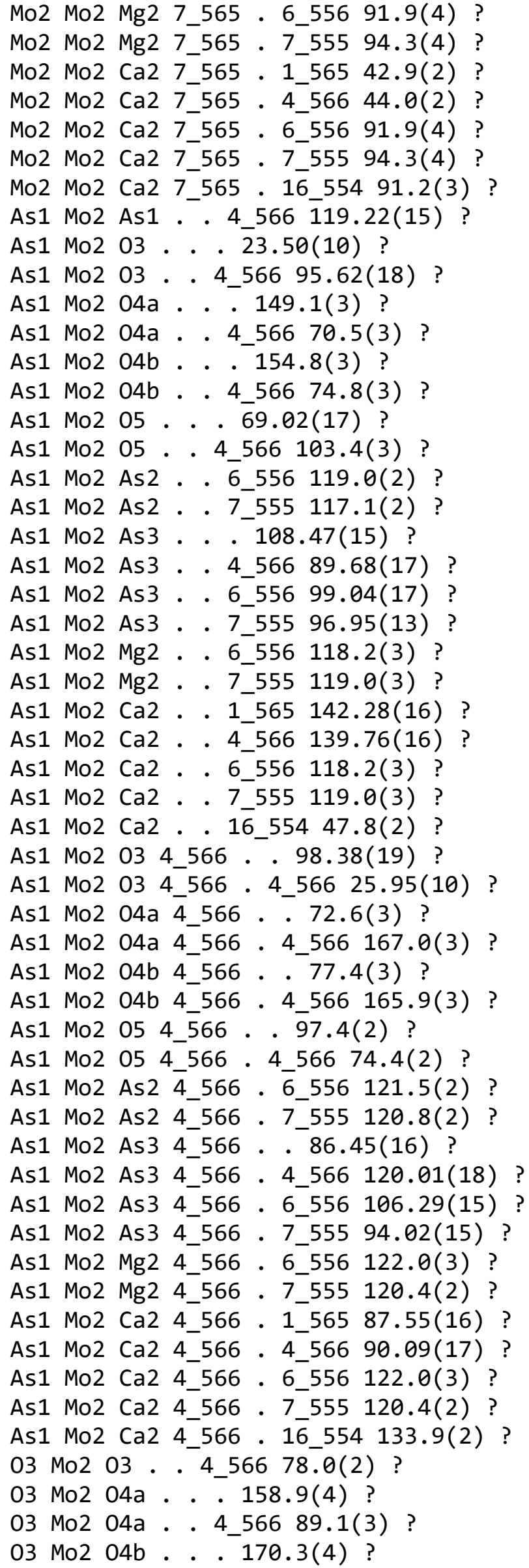




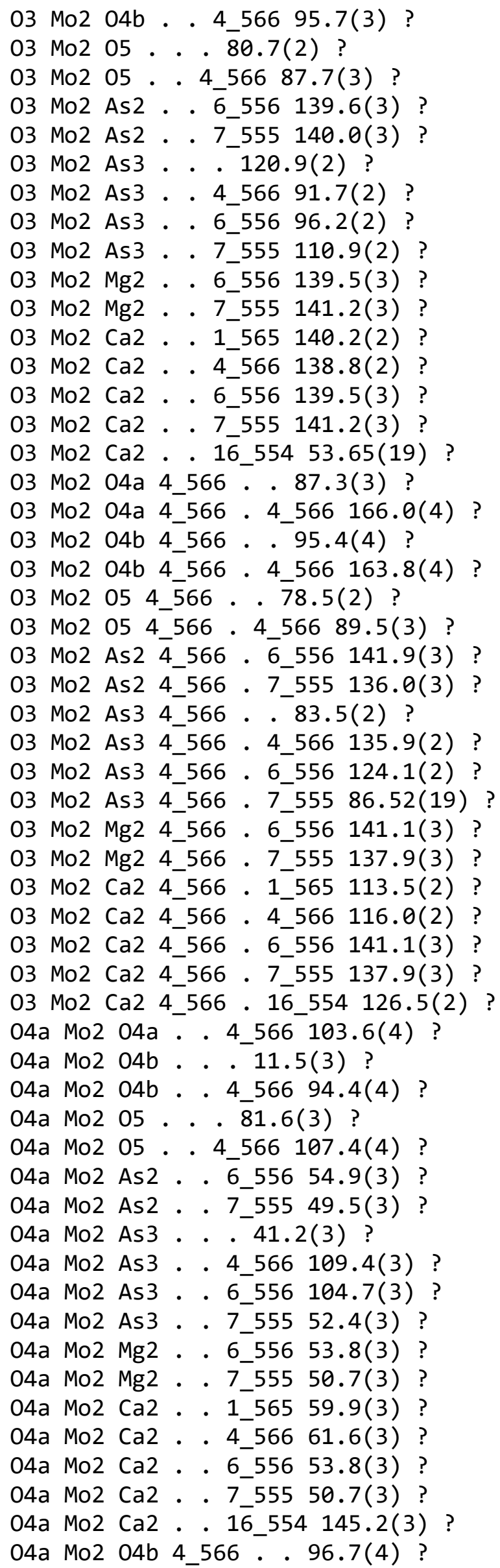




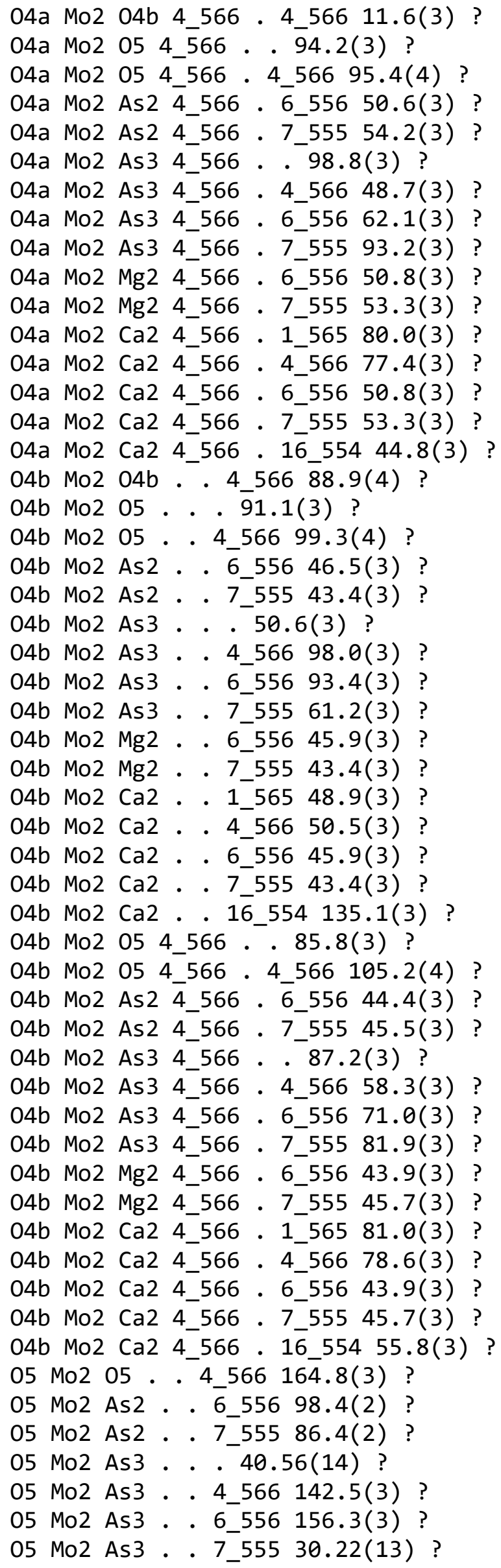




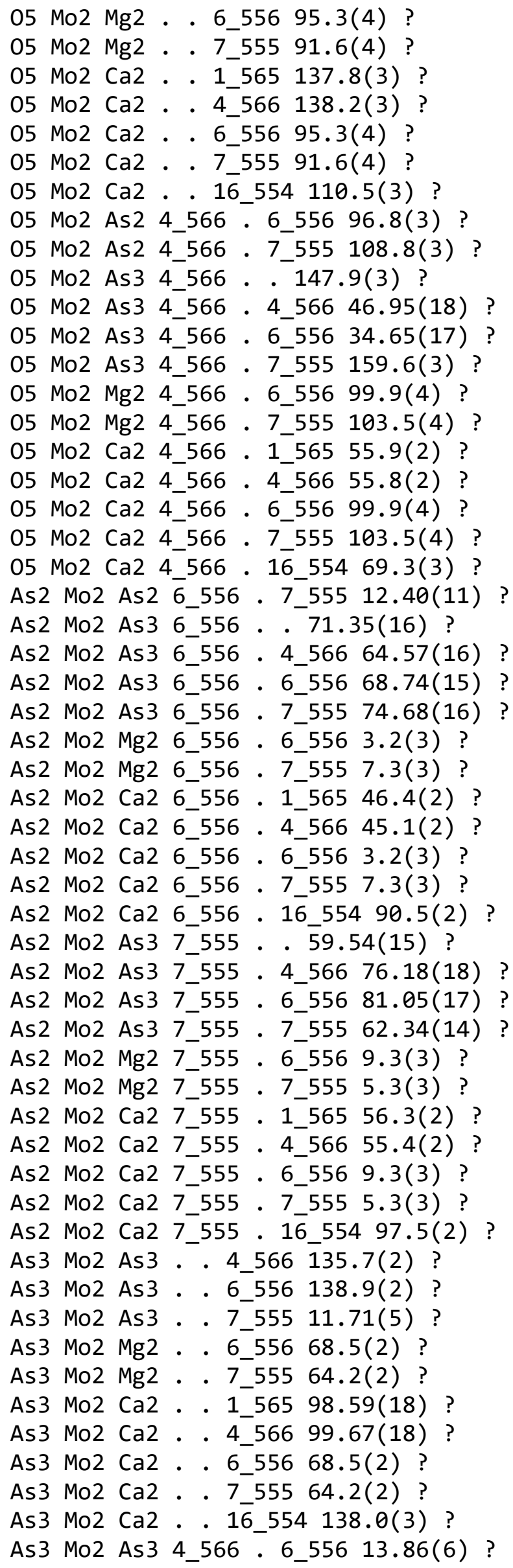




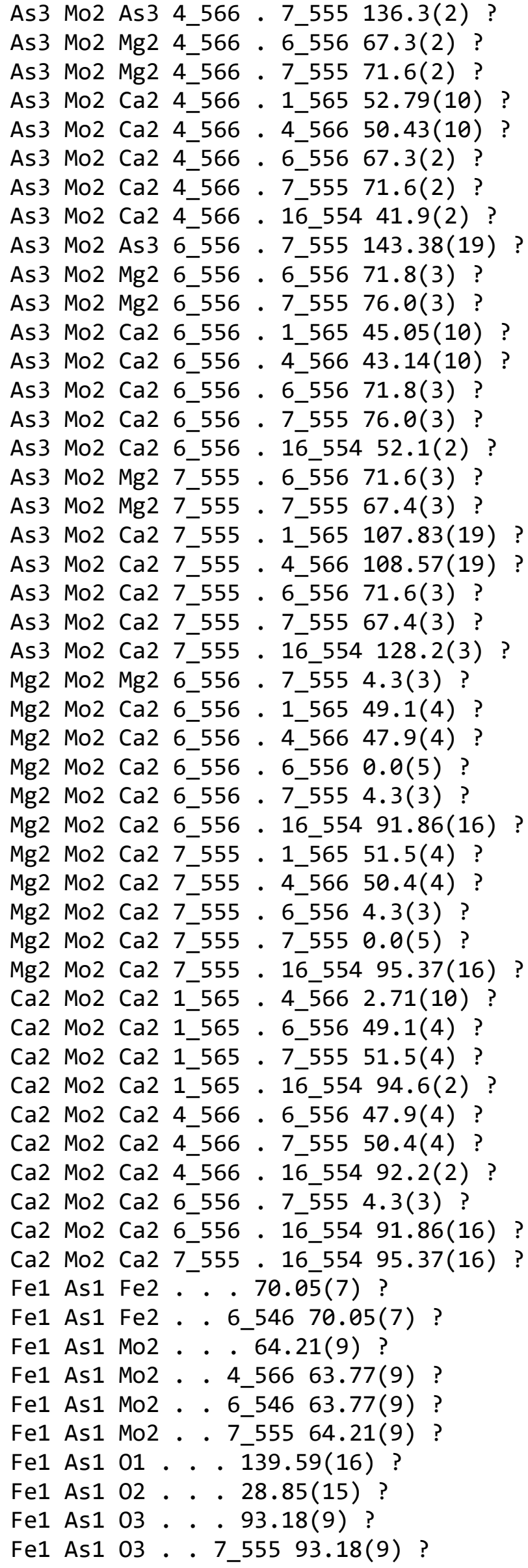




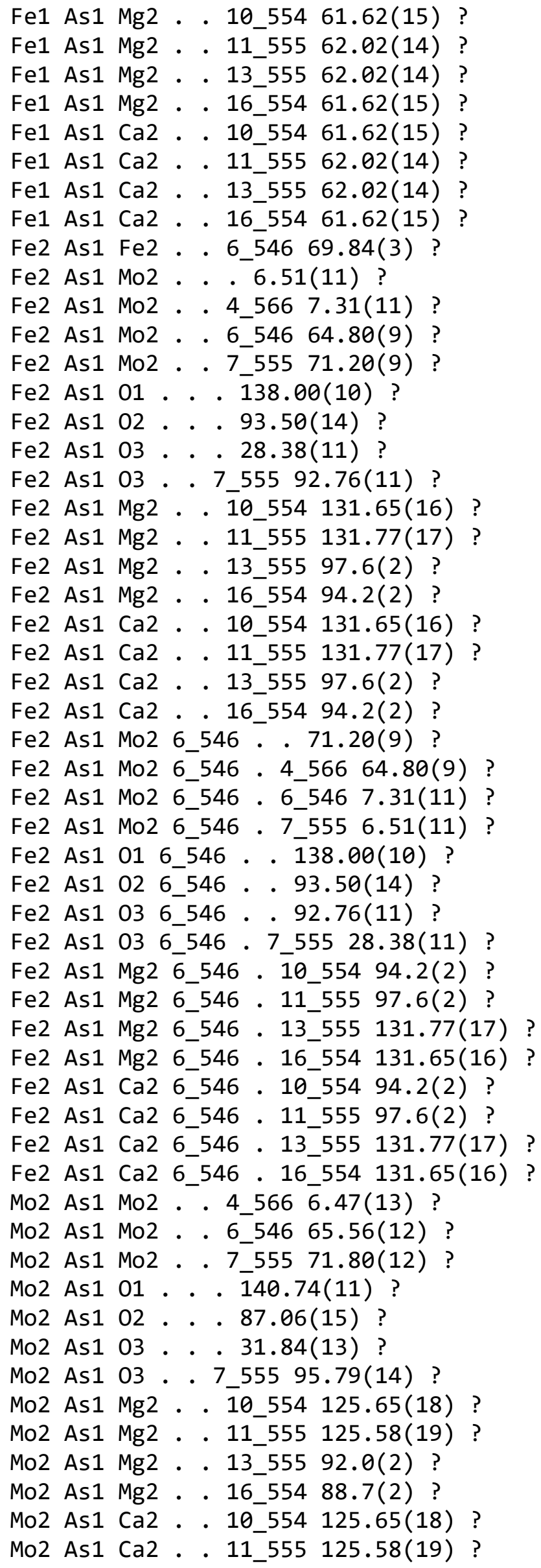




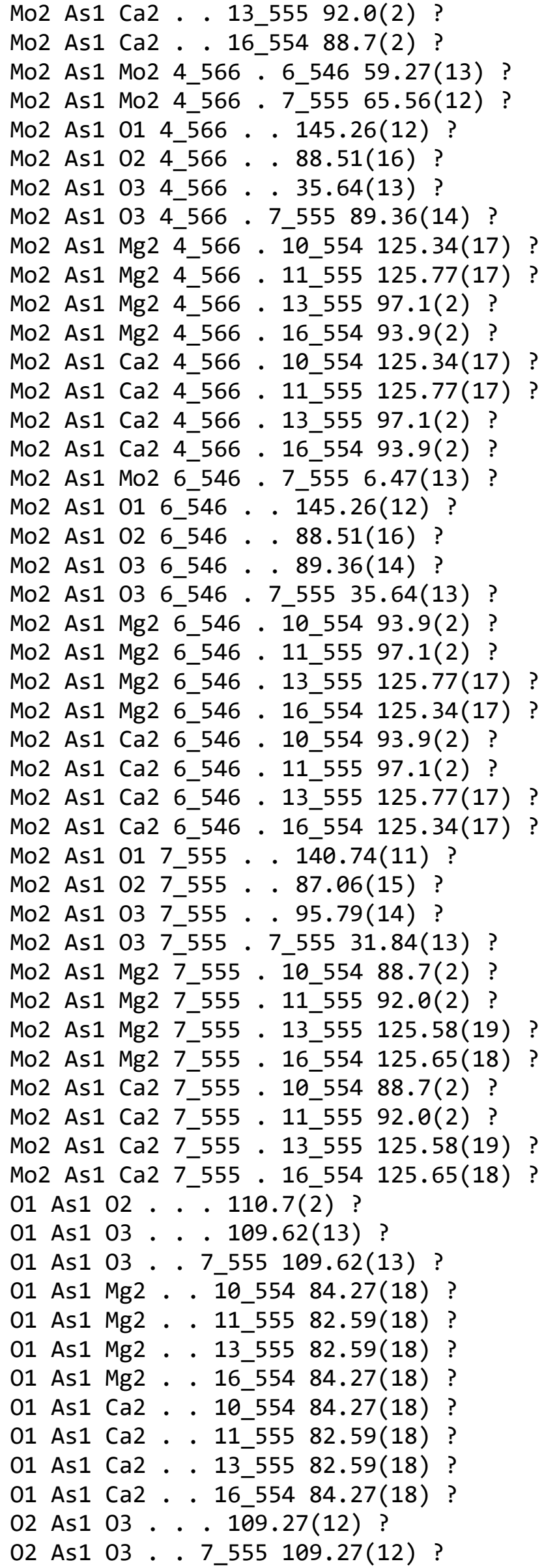




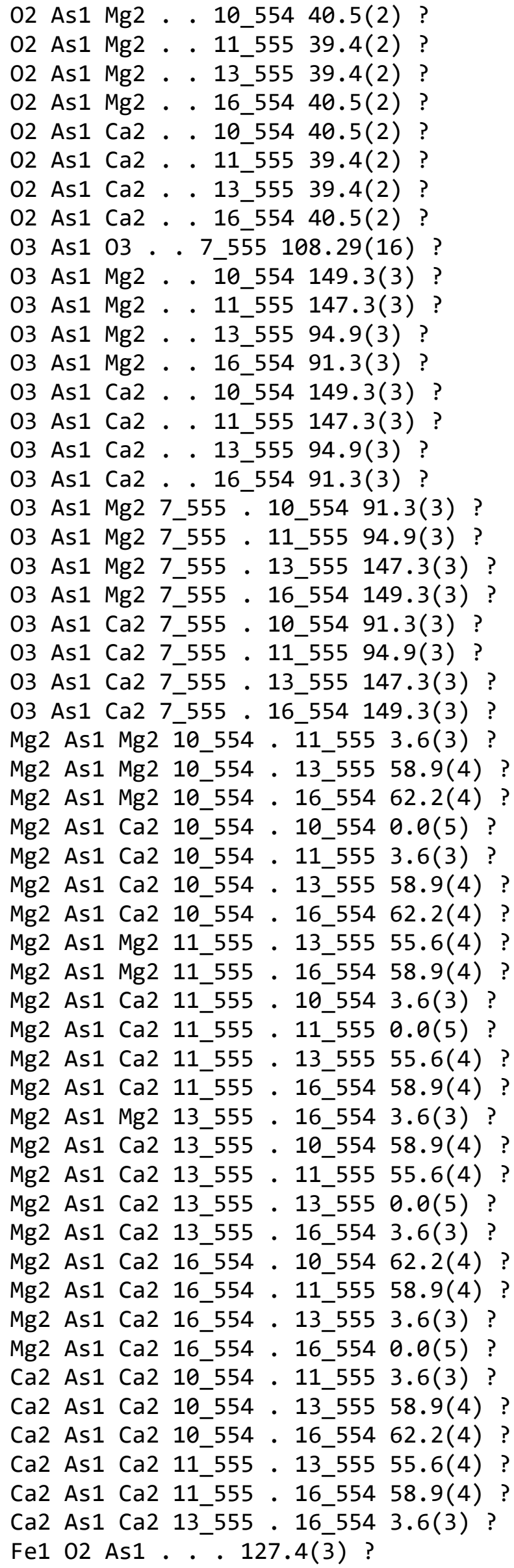




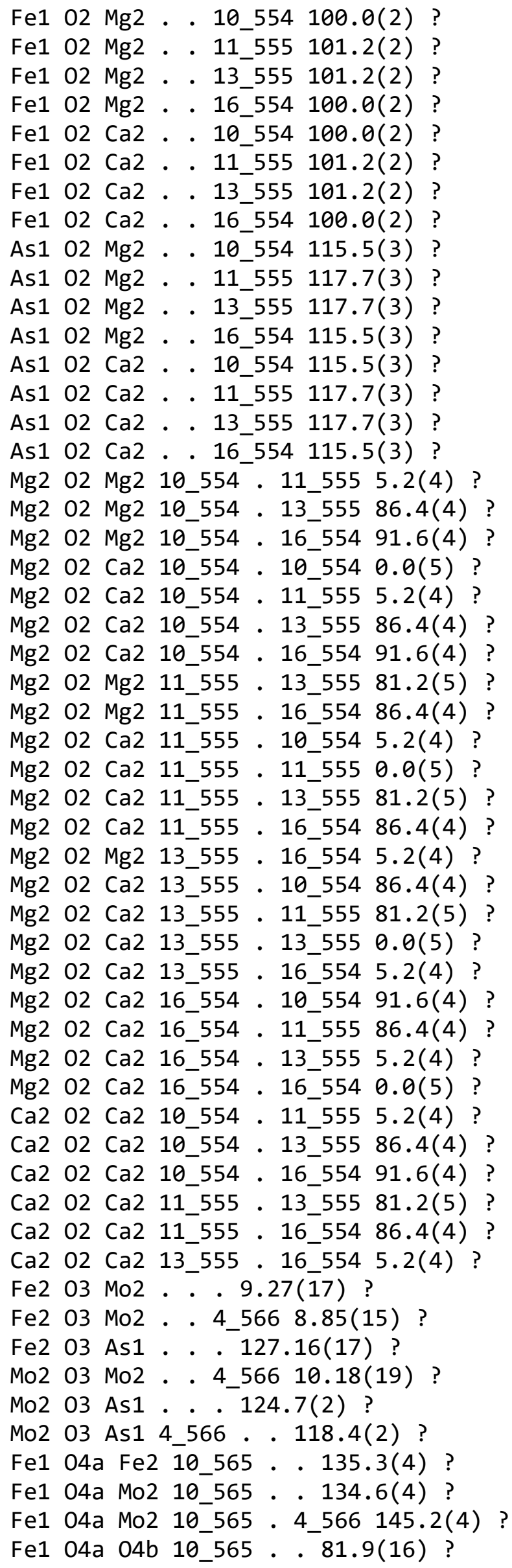




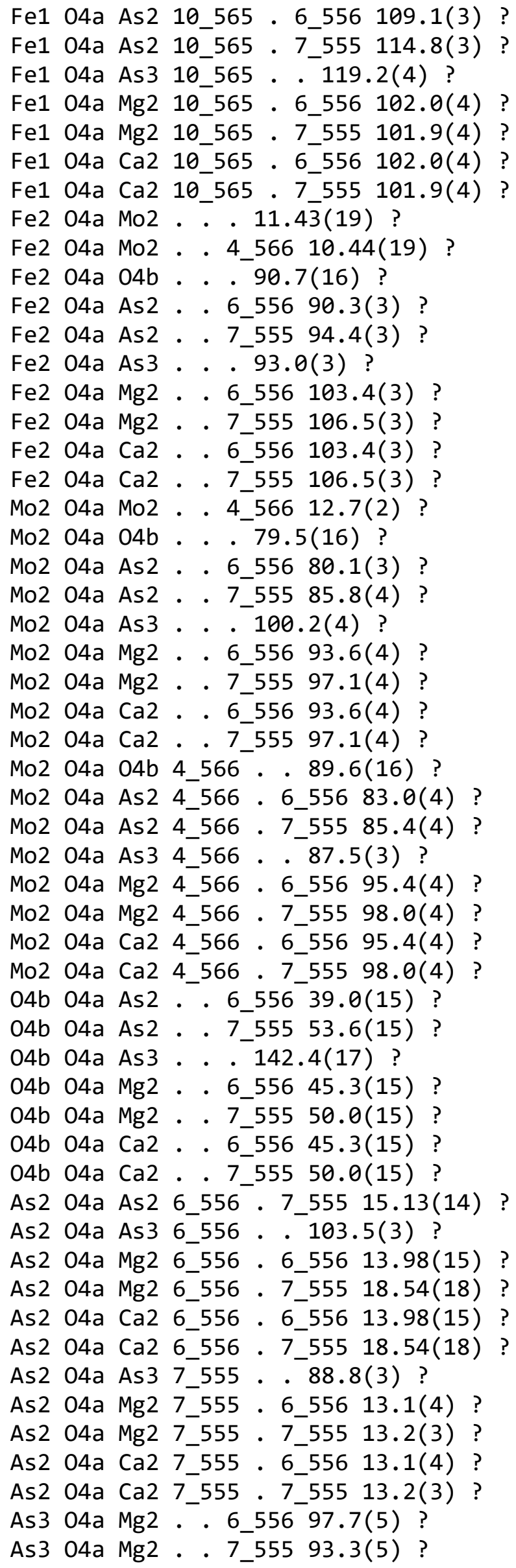




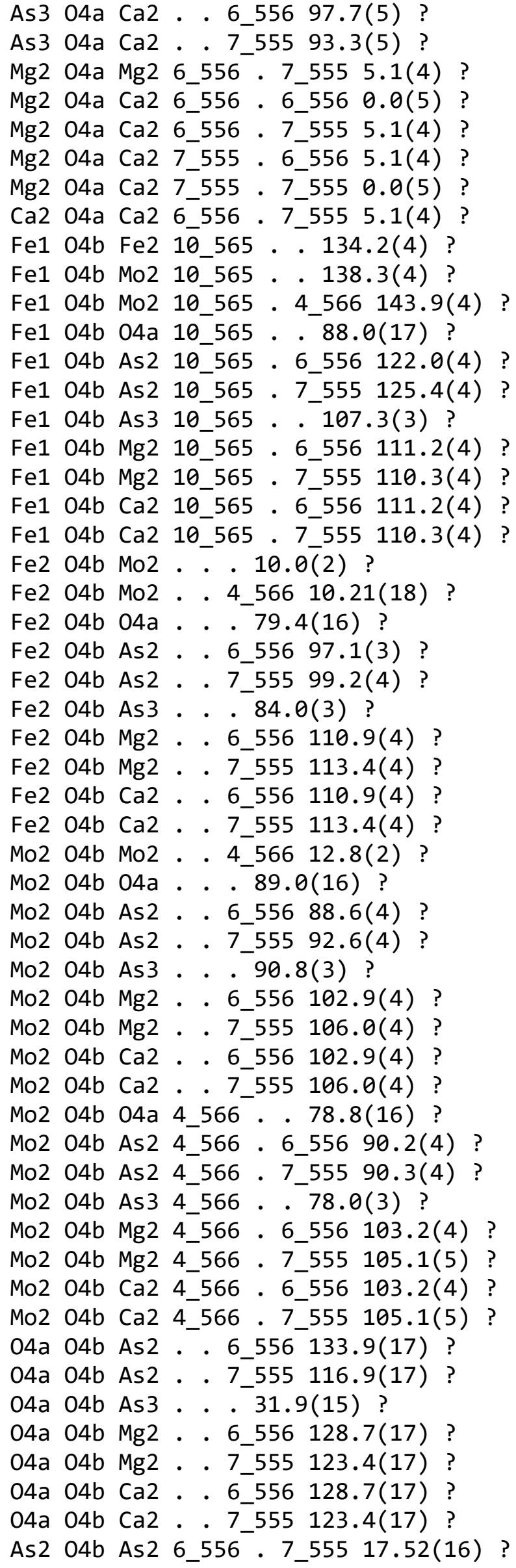




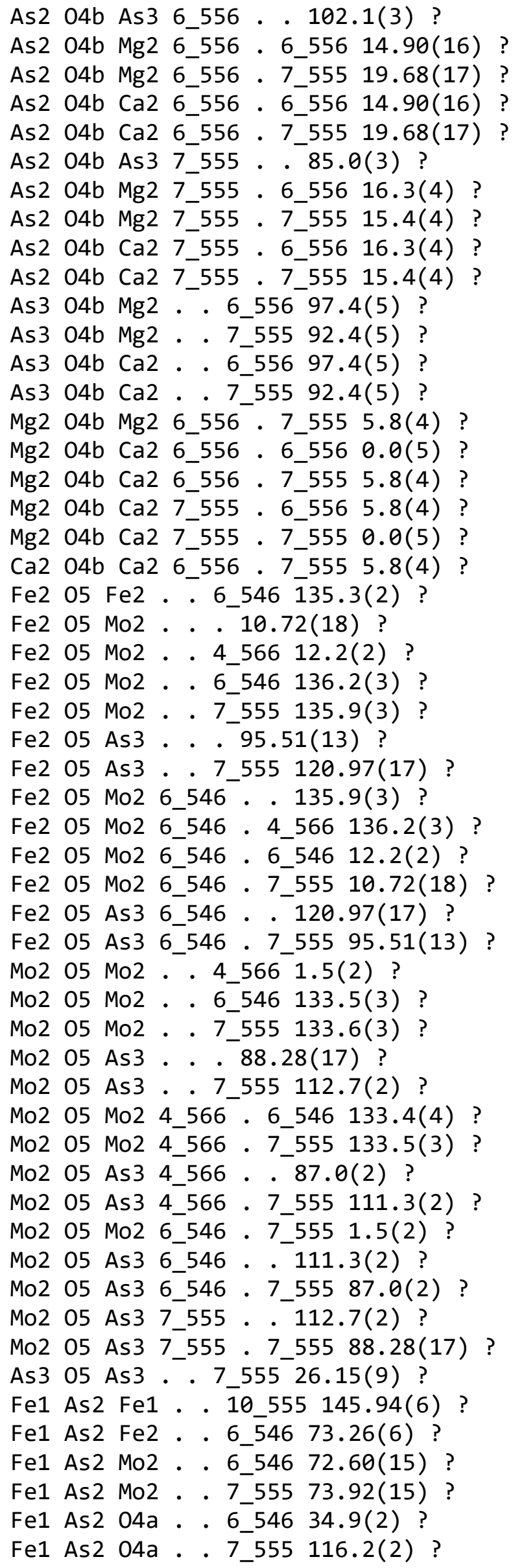




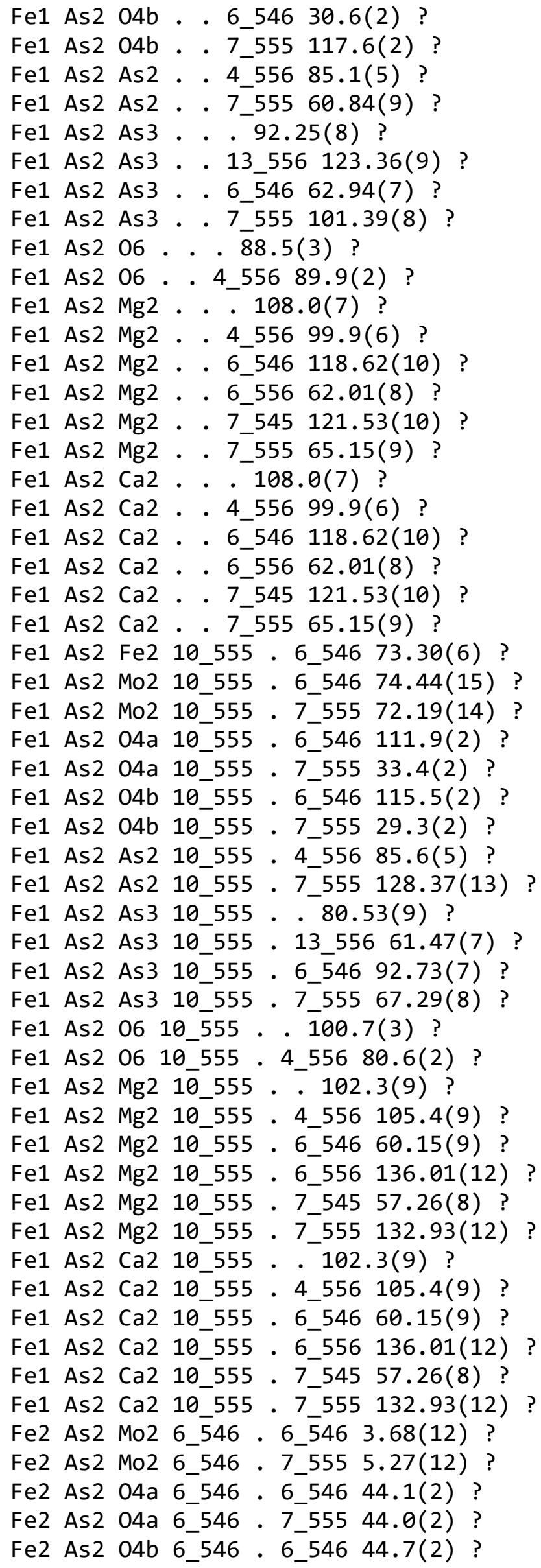




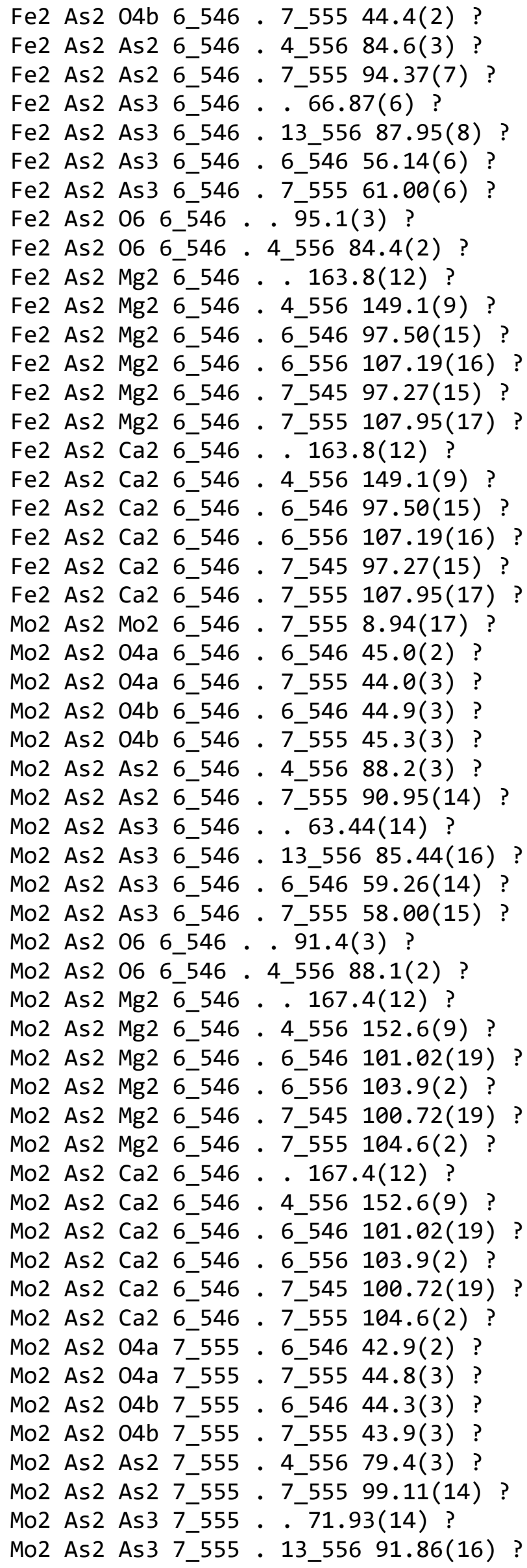




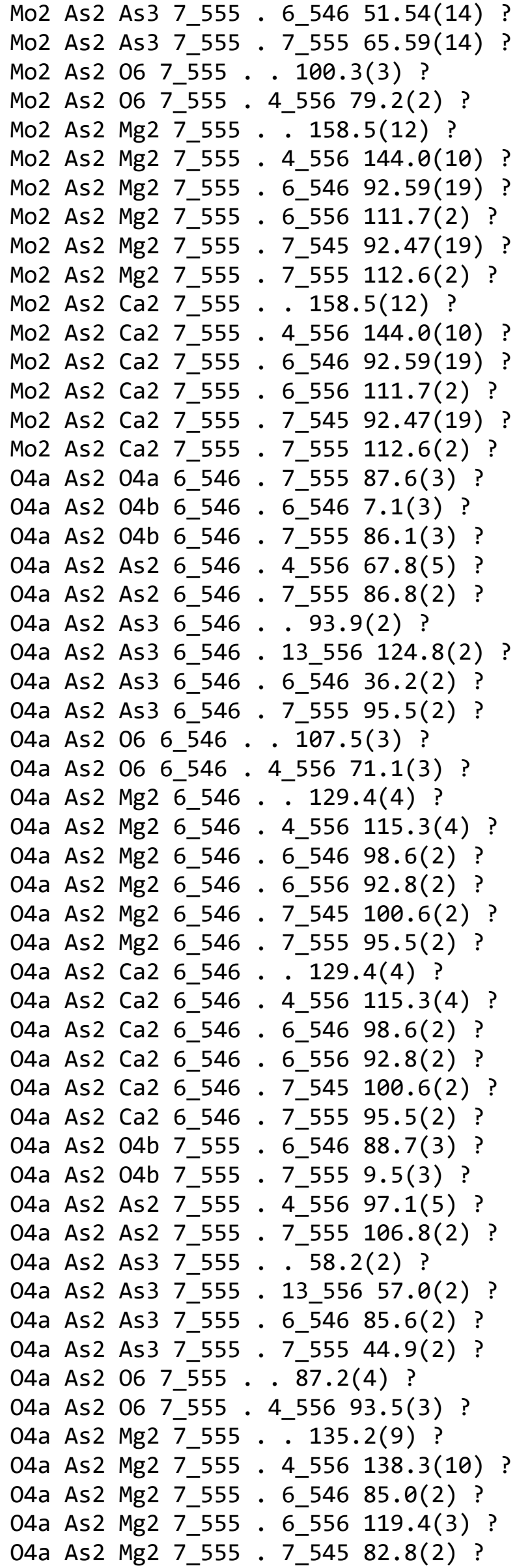




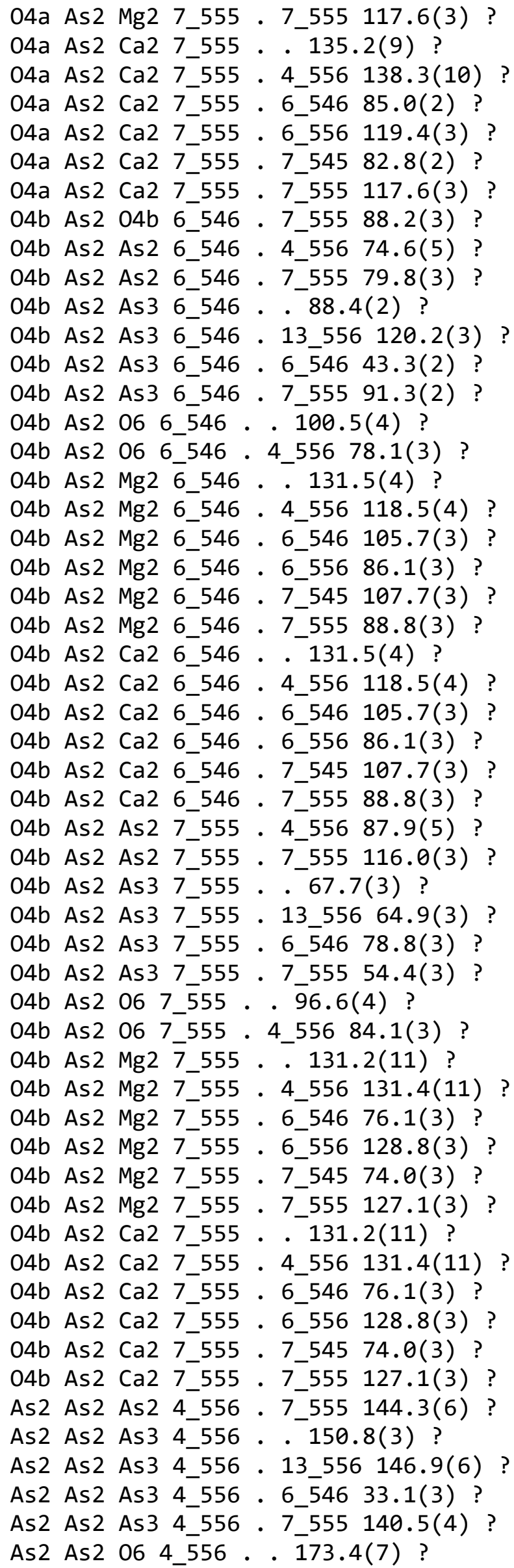




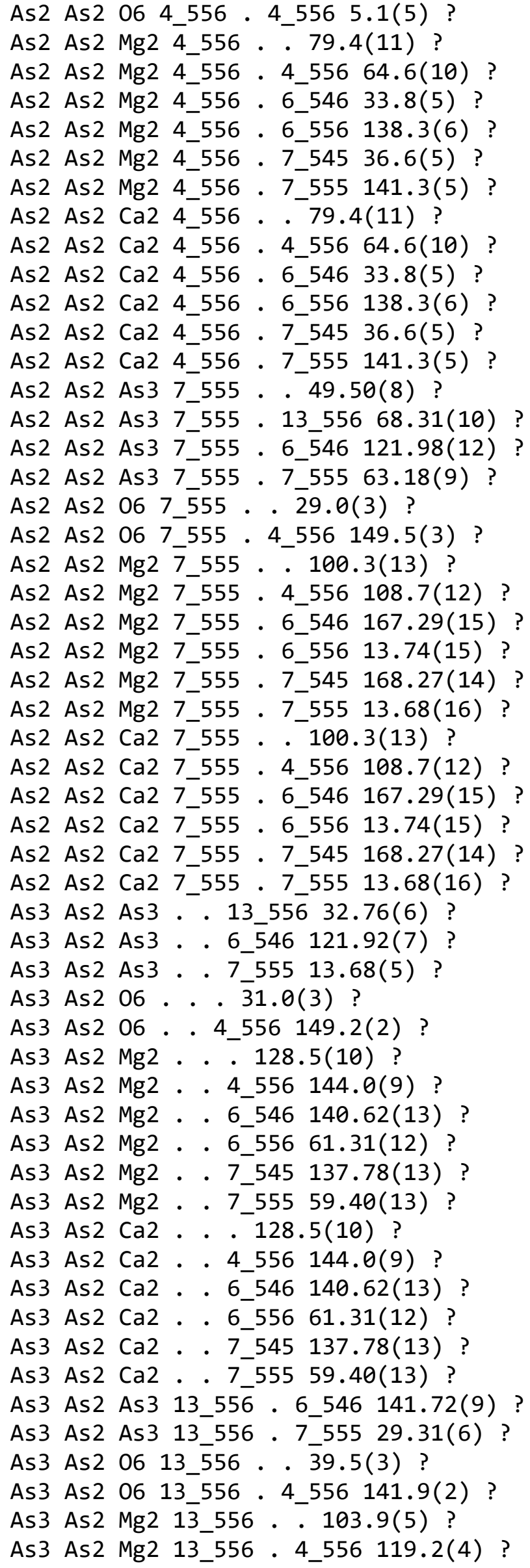




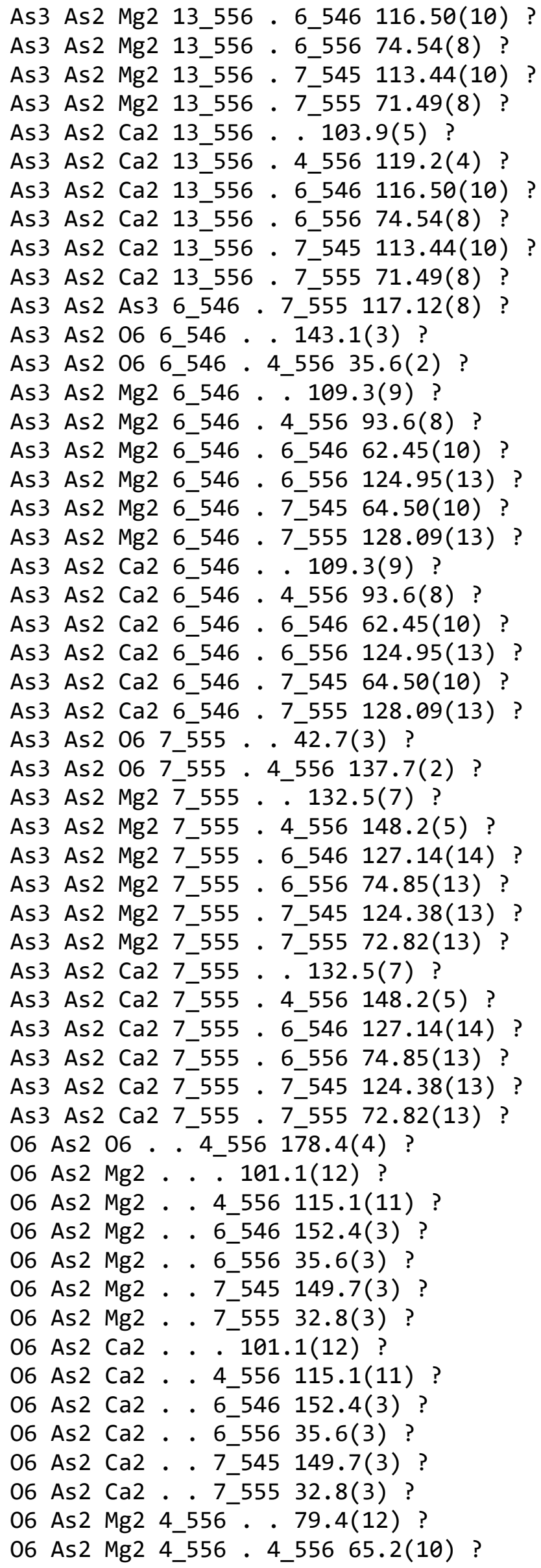


06 As2 Mg2 4_556 . 6_546 29.1(2) ?

06 As2 Mg2 4_556 . 6_556 143.2(2) ?

06 As2 Mg2 4_556 . 7_545 31.8(2) ?

06 As2 Mg2 4_556 . 7_555 146.1(2) ?

06 As2 Ca2 4_556 . 79.4(12) ?

06 As2 Ca2 4_556 . 4_556 65.2(10) ?

06 As2 Ca2 4_556 . 6_546 29.1(2) ?

06 As2 Ca2 4_556 . 6_556 143.2(2) ?

06 As2 Ca2 4_556 . 7_545 31.8(2) ?

06 As2 Ca2 4_556 . 7_555 146.1(2) ?

Mg2 As2 Mg2 . . 4_556 15.8(10) ?

Mg2 As2 Mg2 . 6_546 67.4(13) ?

Mg2 As2 Mg2 . 6_556 86.9(13) ?

Mg2 As2 Mg2 . 7 545 68.0(13) ?

Mg2 As2 Mg2 . . 7_555 86.6(13) ?

Mg2 As2 Ca2 . . $0.0(5)$ ?

Mg2 As2 Ca2 . . 4_556 15.8(10) ?

Mg2 As2 Ca2 . . 6_546 67.4(13) ?

Mg2 As2 Ca2 . . 6_556 86.9(13) ?

Mg2 As2 Ca2 . . 7_545 68.0(13) ?

Mg2 As2 Ca2 . . 7_555 86.6(13) ?

Mg2 As2 Mg2 4_556 . 6_546 58.6(12) ?

Mg2 As2 Mg2 4_556 . 6_556 95.0(12) ?

Mg2 As2 Mg2 4_556 . 7_545 59.9(12) ?

Mg2 As2 Mg2 4_556 . 7_555 95.4(12) ?

Mg2 As2 Ca2 4_556 . . 15.8(10) ?

Mg2 As2 Ca2 4_556 . 4_556 0.0(5) ?

Mg2 As2 Ca2 4_556 . 6_546 58.6(12) ?

Mg2 As2 Ca2 4_556 . 6_556 95.0(12) ?

Mg2 As2 Ca2 4_556 . 7_545 59.9(12) ?

Mg2 As2 Ca2 4_556 . 7_555 95.4(12) ?

Mg2 As2 Mg2 6_546 . 6_556 153.5(2) ?

Mg2 As2 Mg2 6_546.7_545 3.08(4) ?

Mg2 As2 Mg2 6_546 . 7_555 153.8(2) ?

Mg2 As2 Ca2 6_546. . 67.4(13) ?

Mg2 As2 Ca2 6_546 . 4_556 58.6(12) ?

Mg2 As2 Ca2 6_546 . 6_546 0.0(5) ?

Mg2 As2 Ca2 6_546 . 6_556 153.5(2) ?

Mg2 As2 Ca2 6_546 . 7_545 3.08(4) ?

Mg2 As2 Ca2 6_546 . 7_555 153.8(2) ?

Mg2 As2 Mg2 6_556 . 7_545 154.7(2) ?

Mg2 As2 Mg2 6_556 . 7_555 3.27(3) ?

Mg2 As2 Ca2 6_556 . . 86.9(13) ?

Mg2 As2 Ca2 6_556 . 4_556 95.0(12) ?

Mg2 As2 Ca2 6_556 . 6_546 153.5(2) ?

Mg2 As2 Ca2 6_556 . 6_556 0.0(5) ?

Mg2 As2 Ca2 6_556 . 7_545 154.7(2) ?

Mg2 As2 Ca2 6_556 . 7_555 3.27(3) ?

Mg2 As2 Mg2 7_545 . 7_555 154.6(2) ?

Mg2 As2 Ca2 7_545 . .68.0(13) ?

Mg2 As2 Ca2 7_545 . 4_556 59.9(12) ?

Mg2 As2 Ca2 7_545 . 6_546 3.08(4) ?

Mg2 As2 Ca2 7_545 . 6_556 154.7(2) ?

Mg2 As2 Ca2 7_545 . 7_545 0.0(5)? 


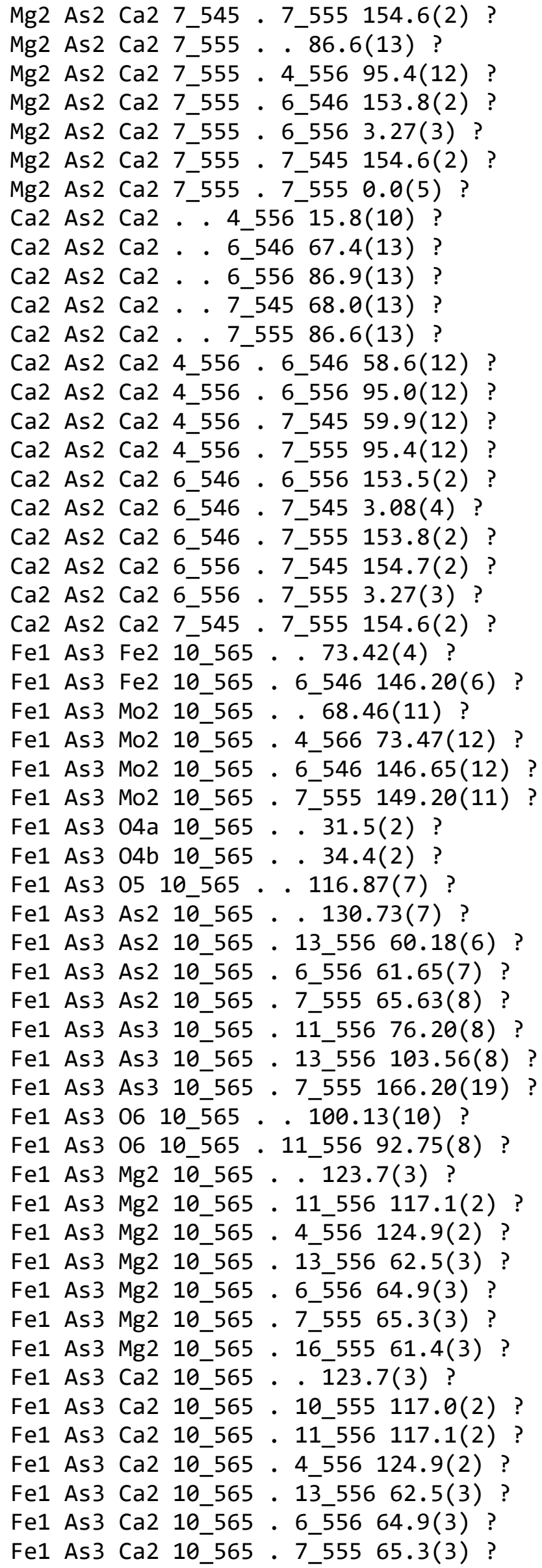




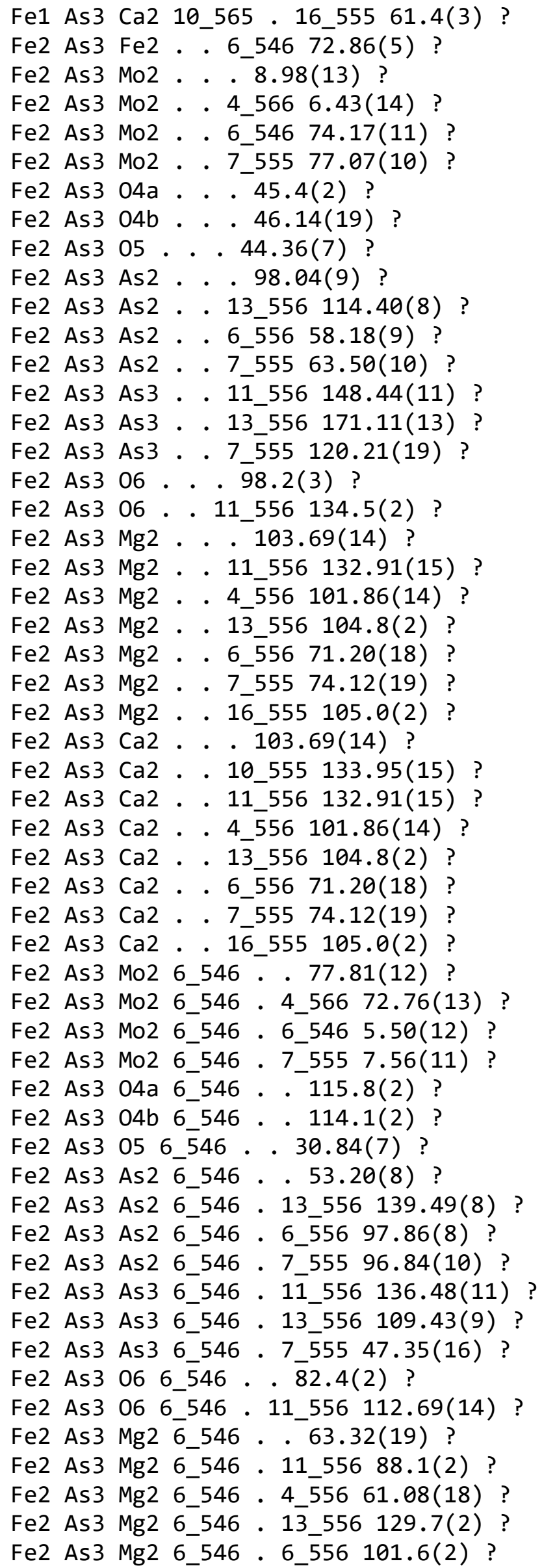




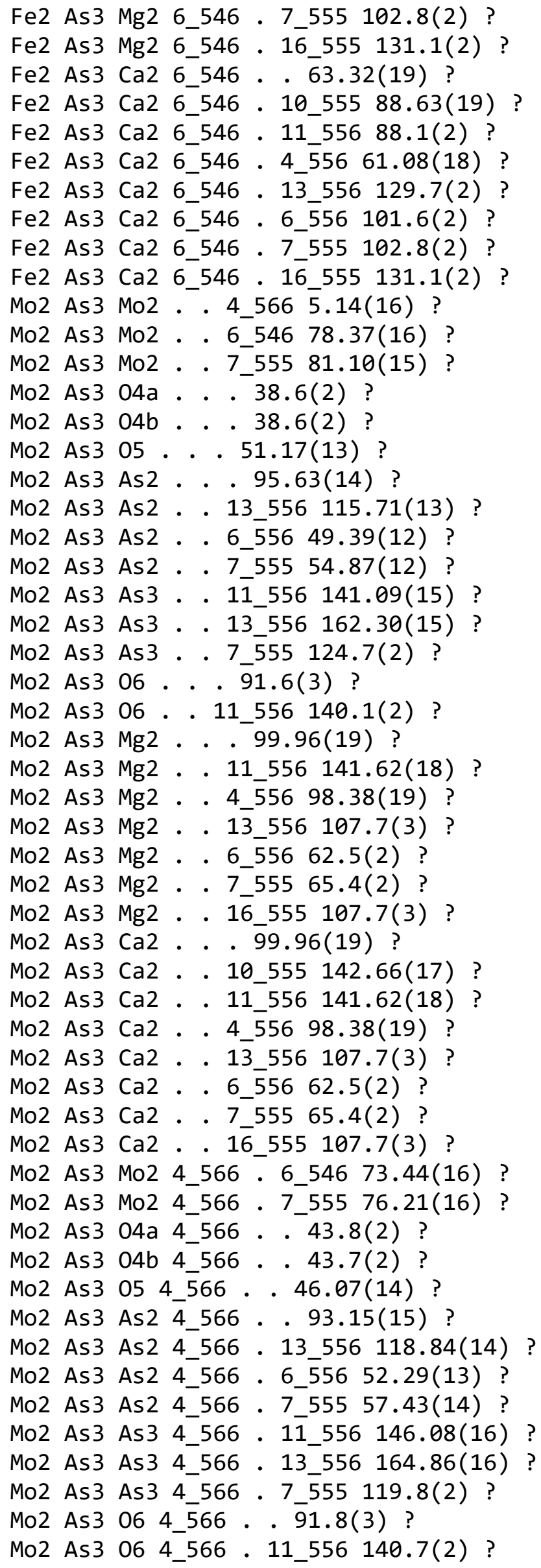




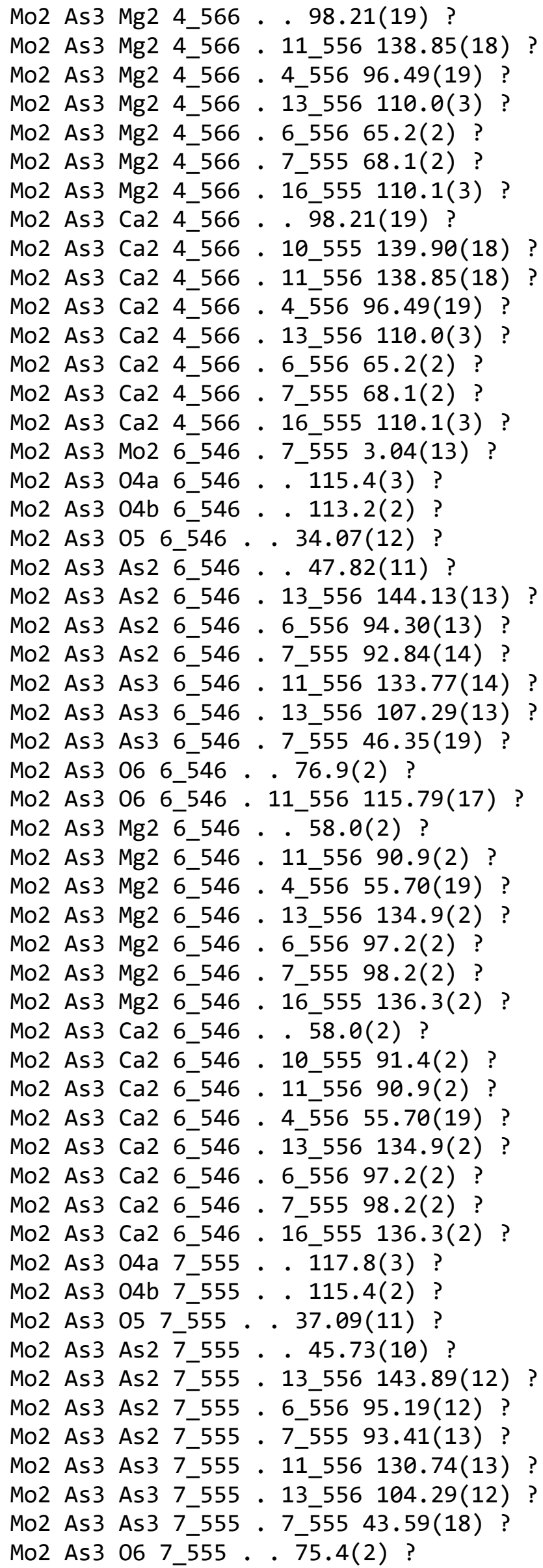




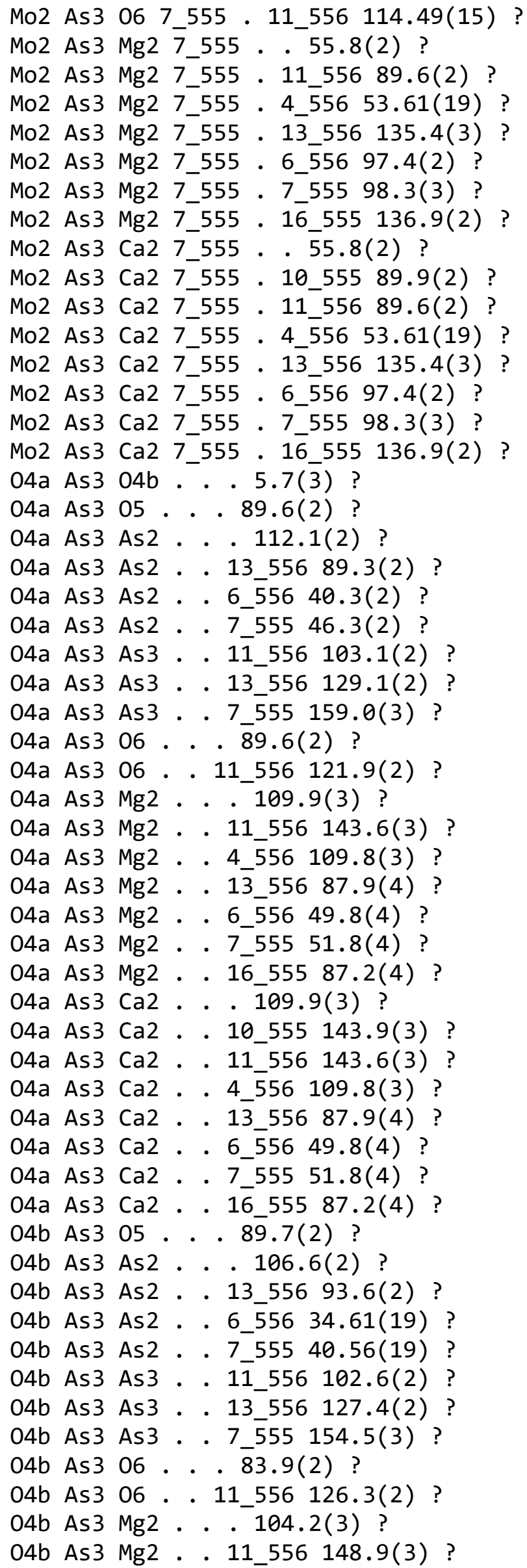




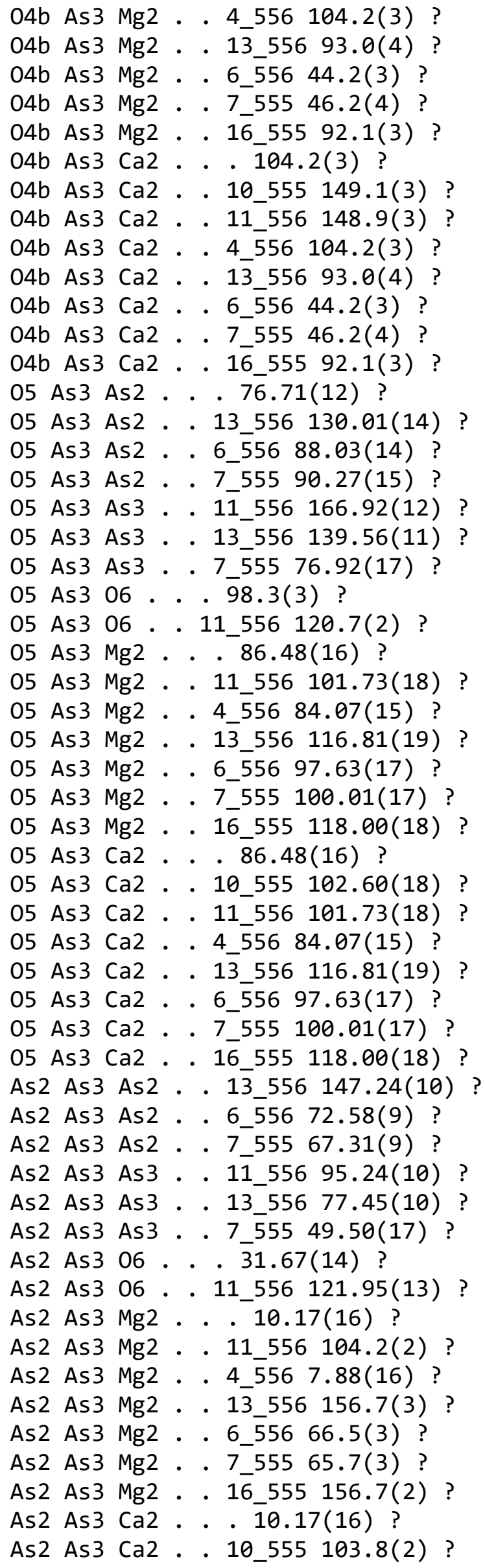




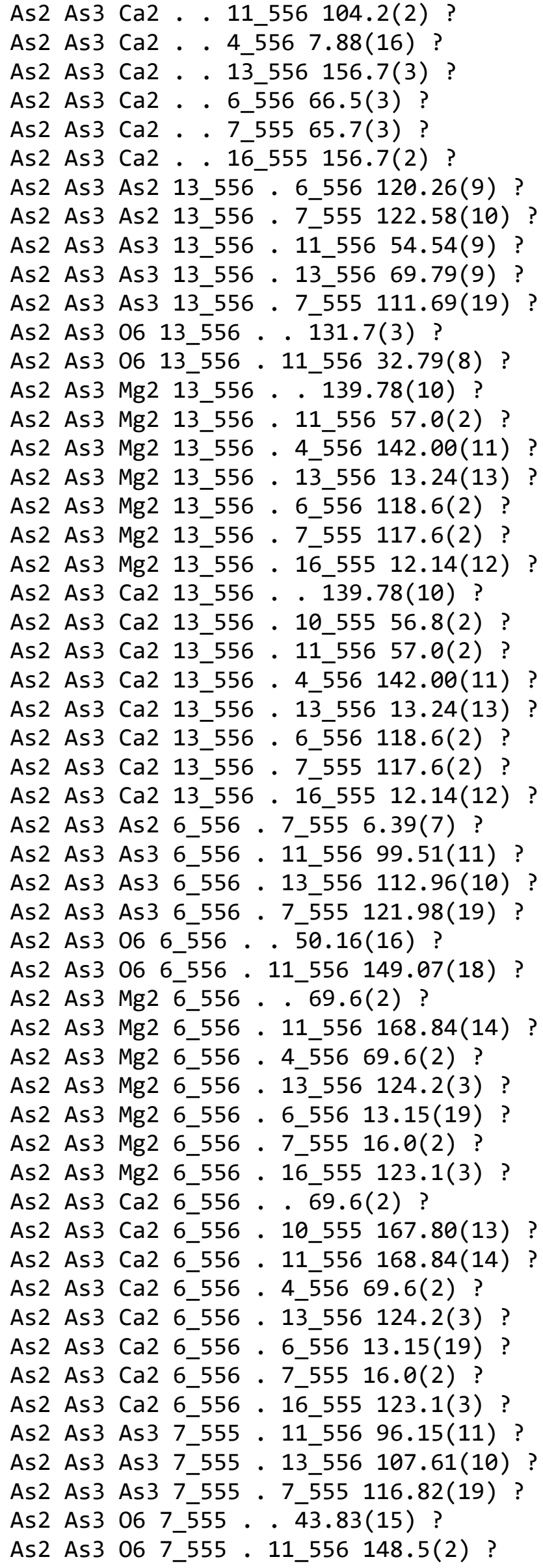




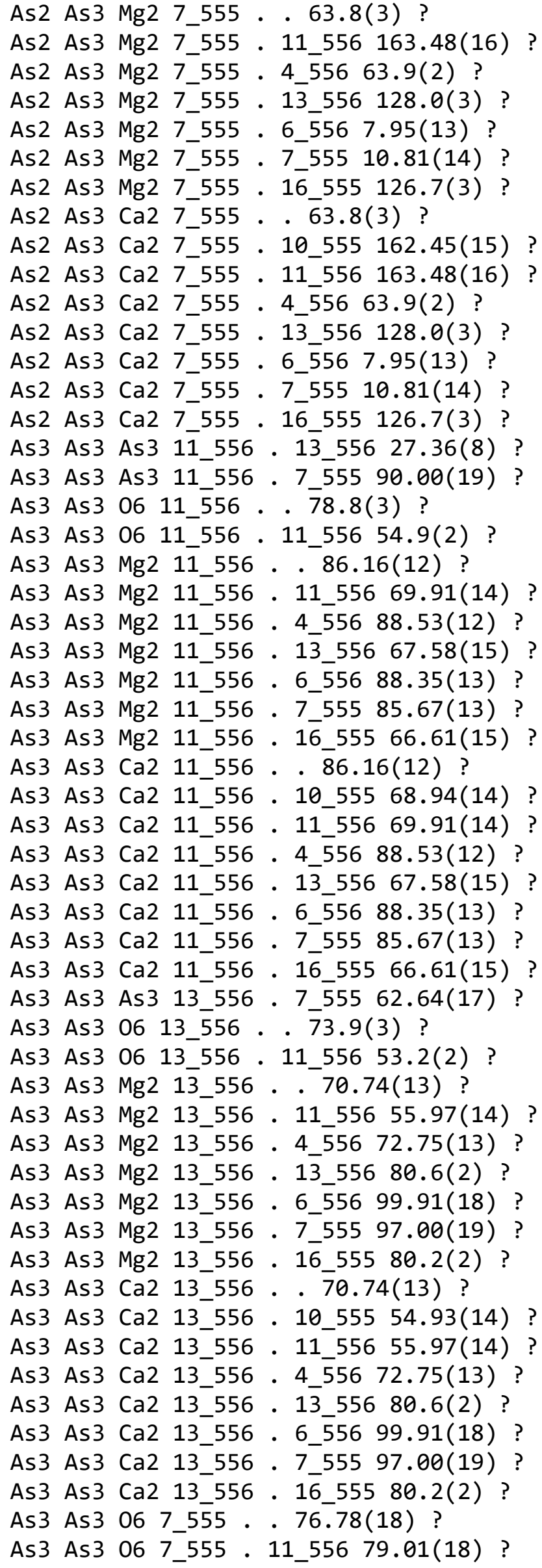




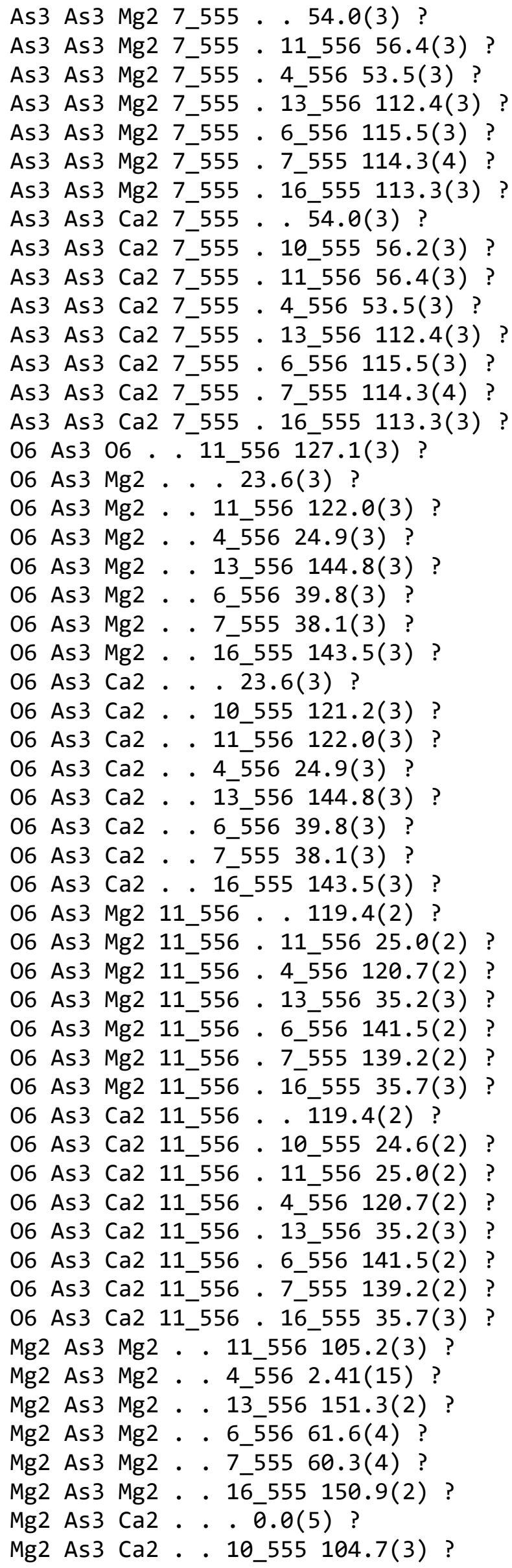




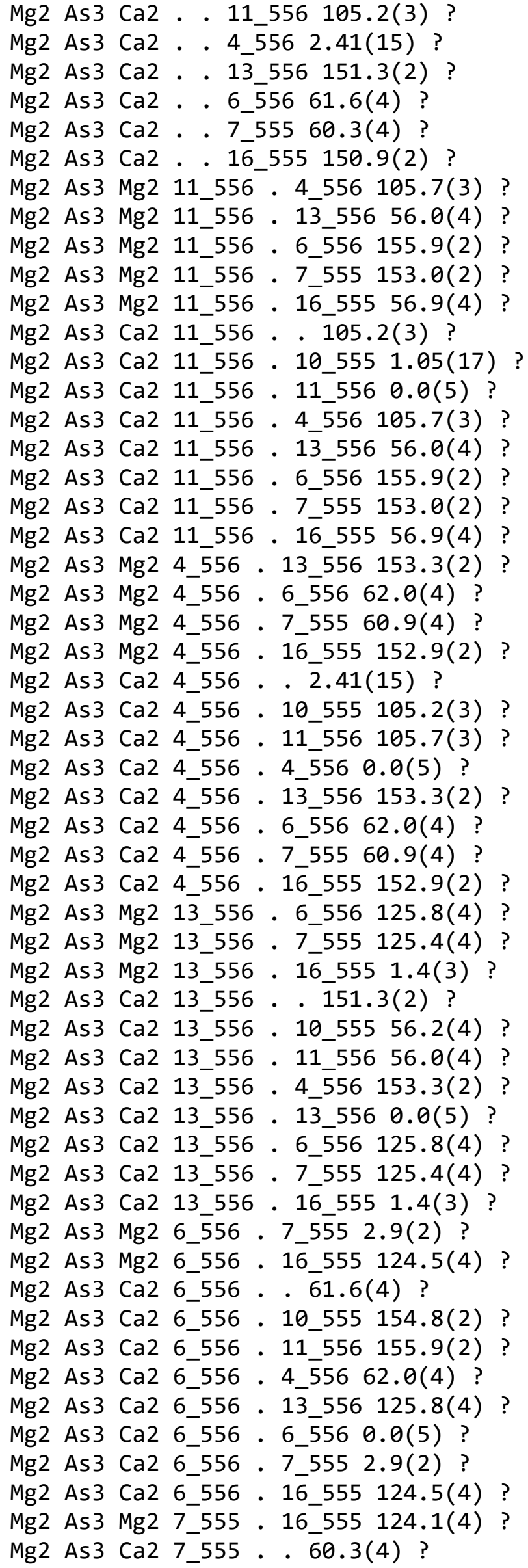




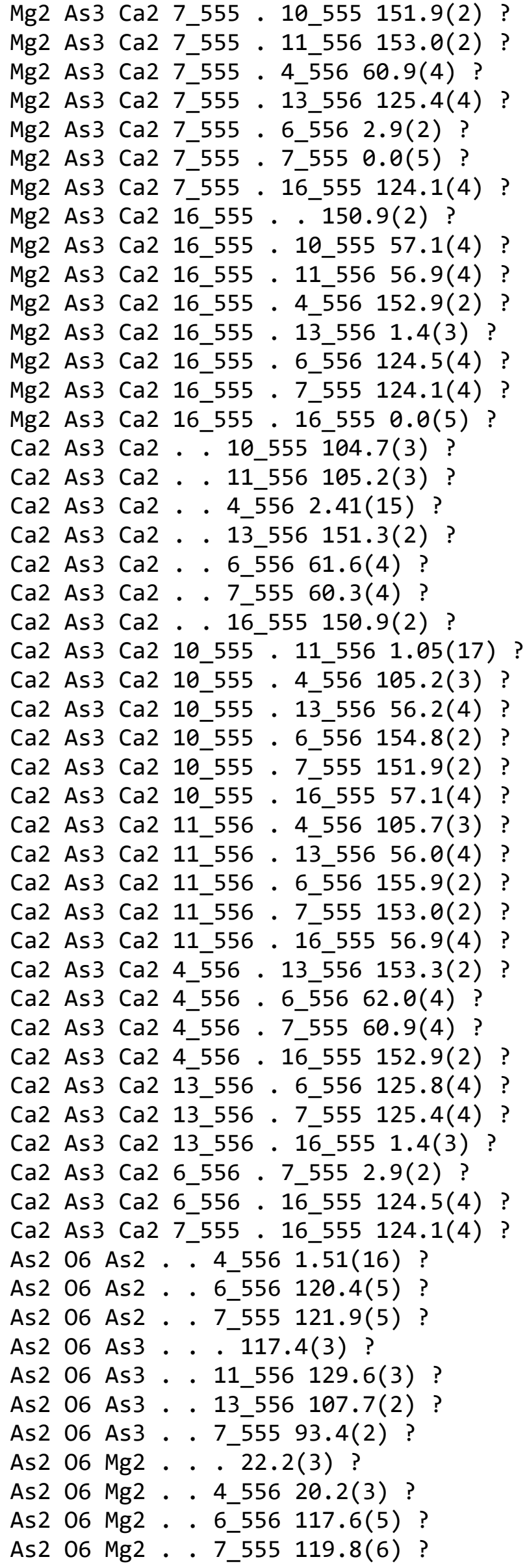




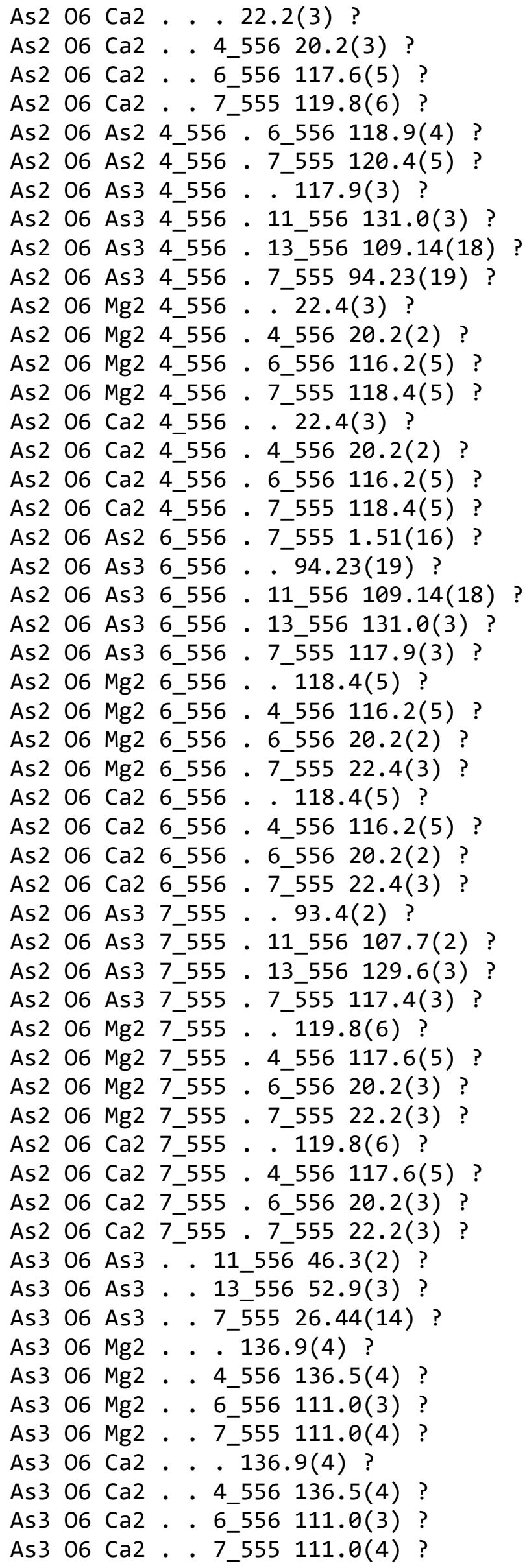




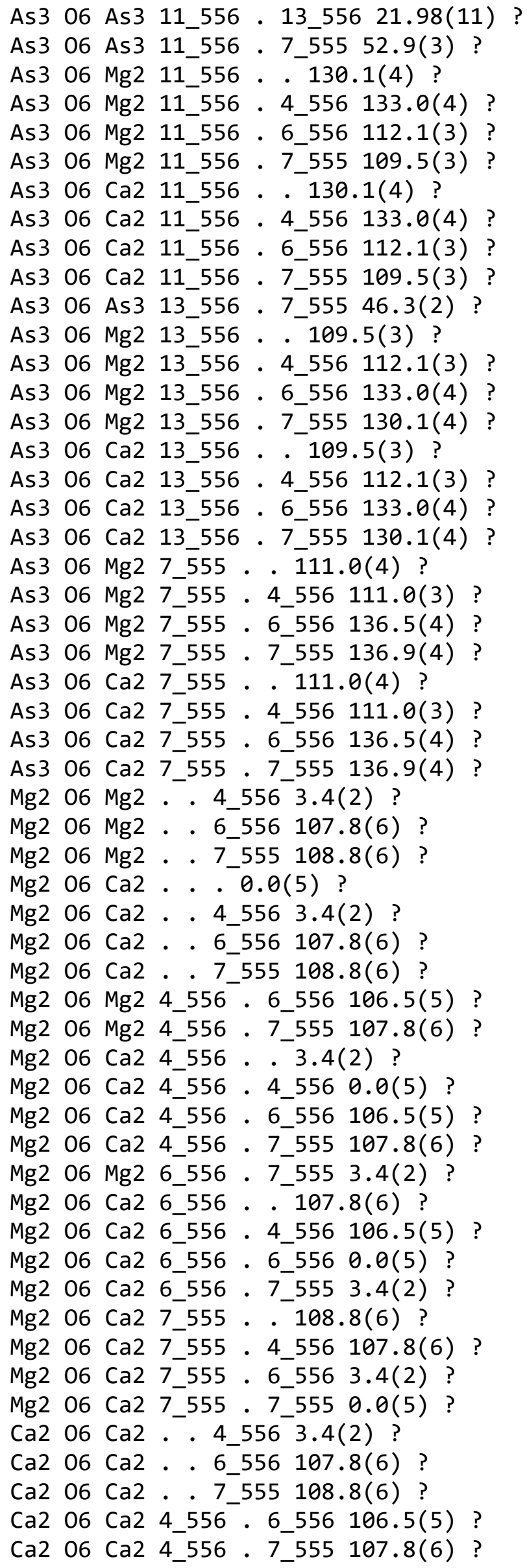




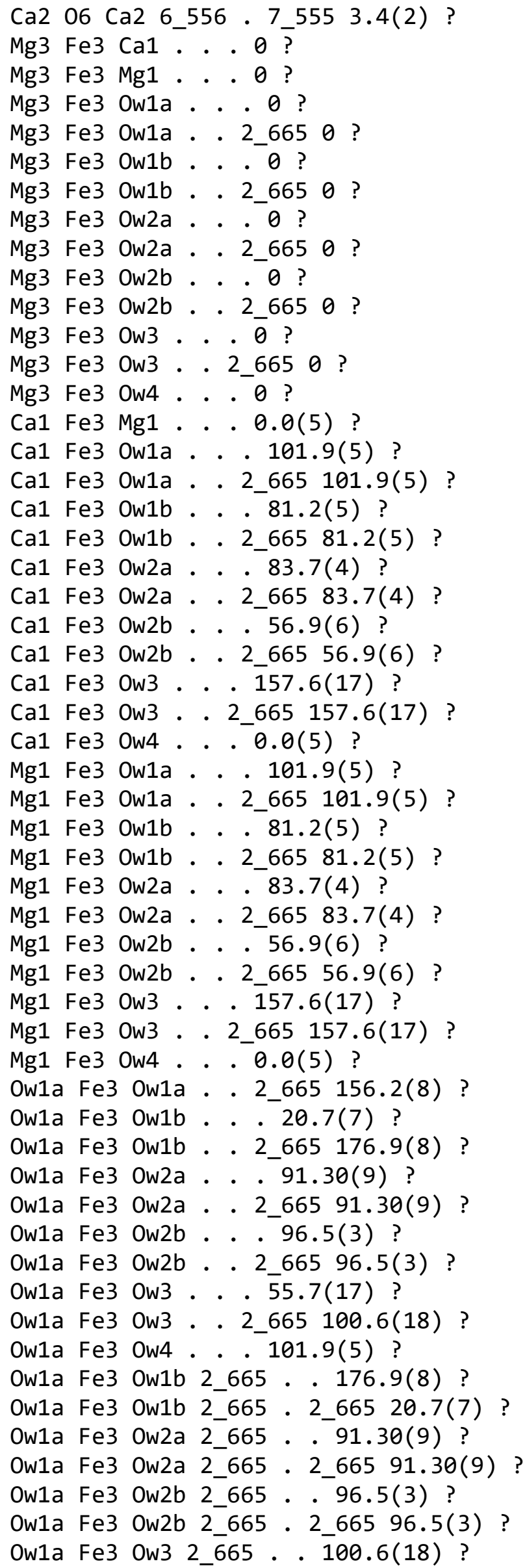




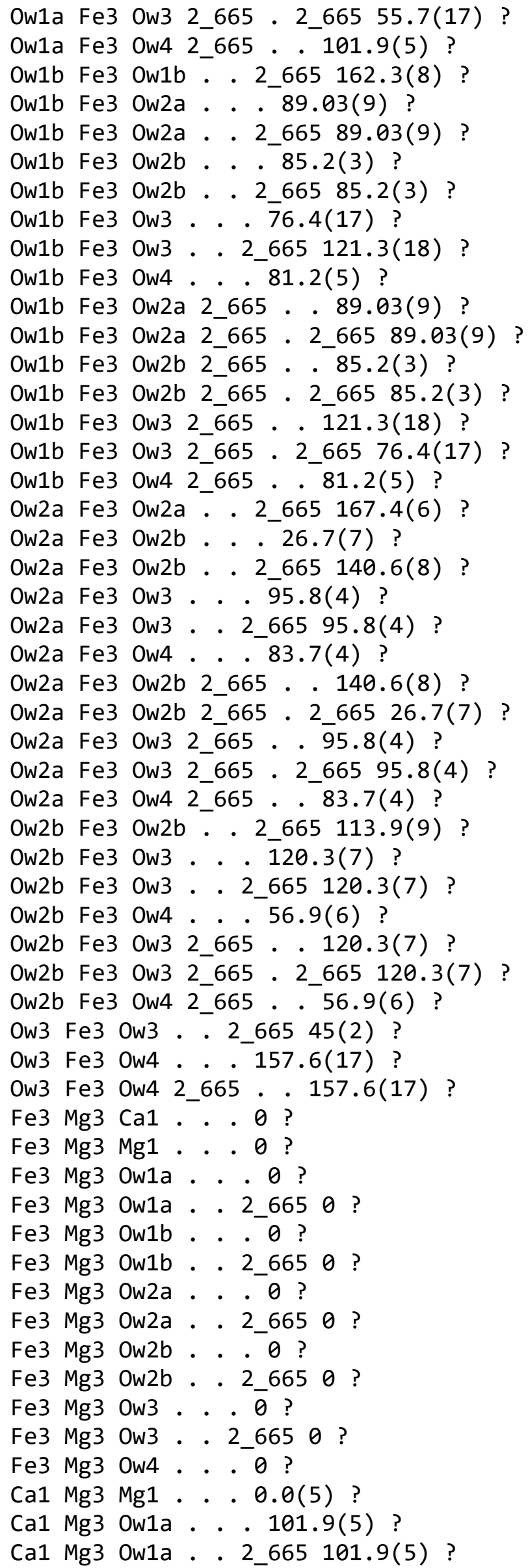




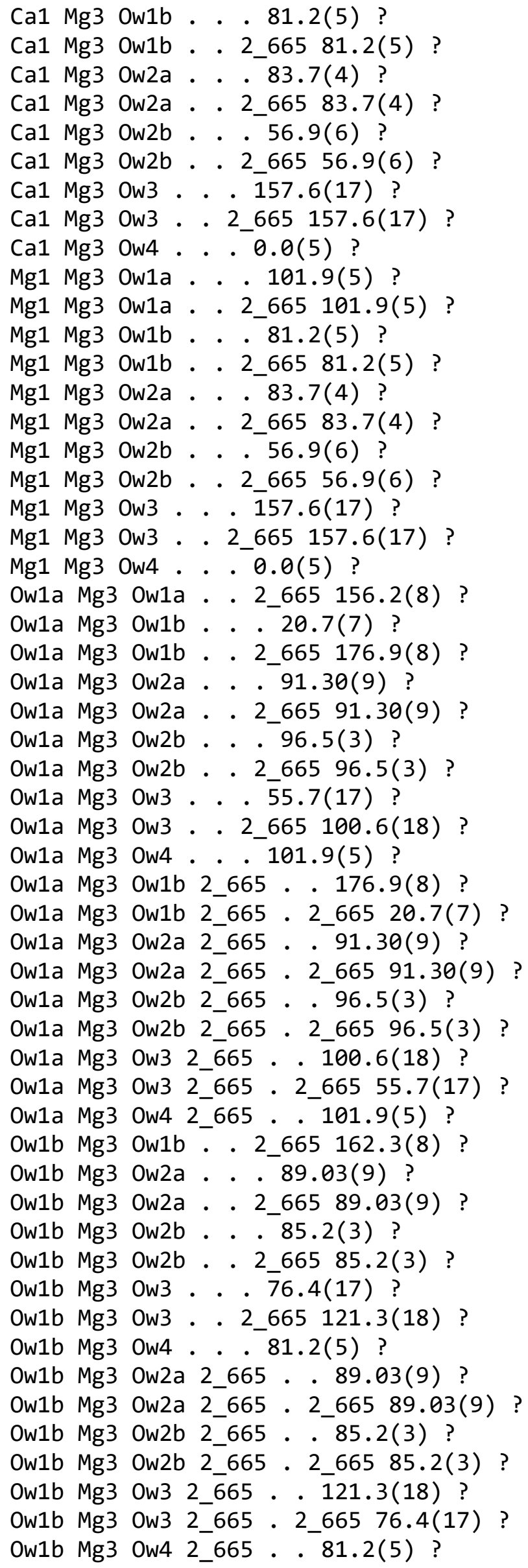




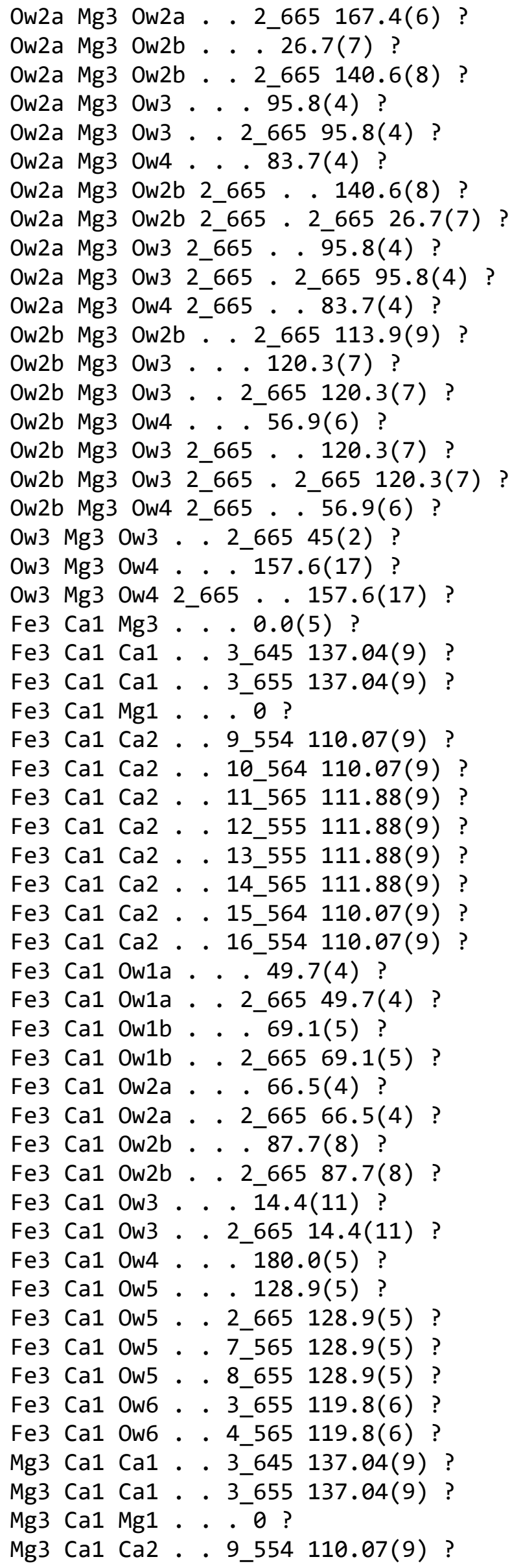




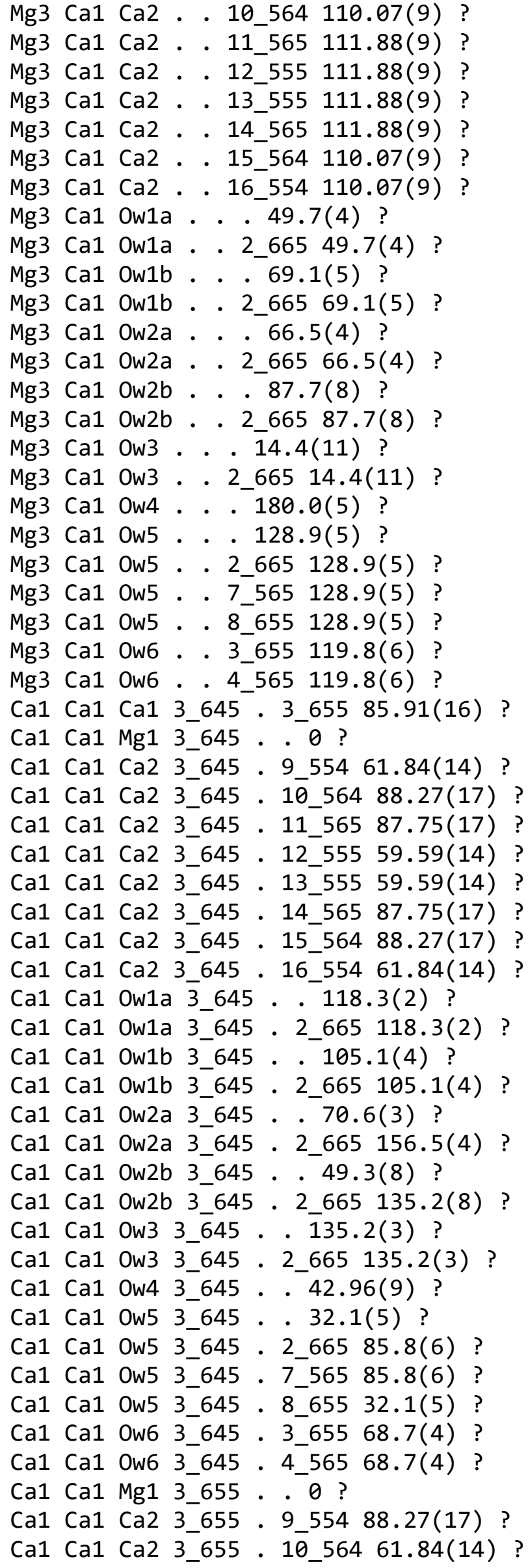




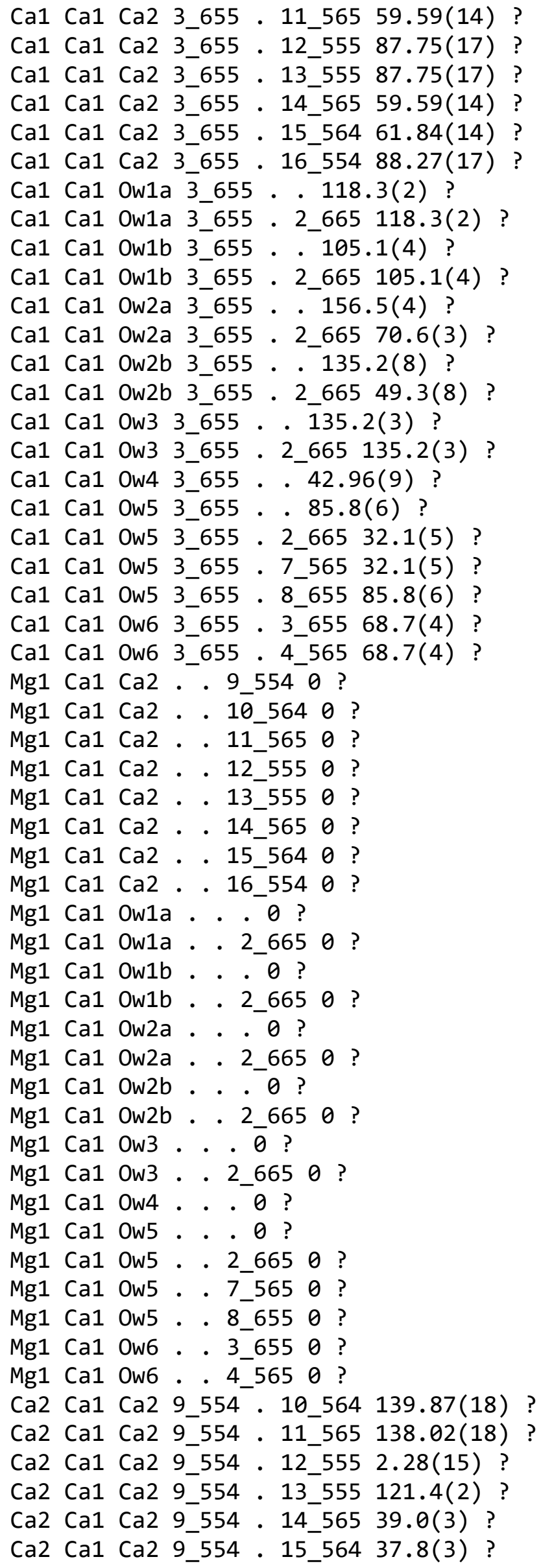




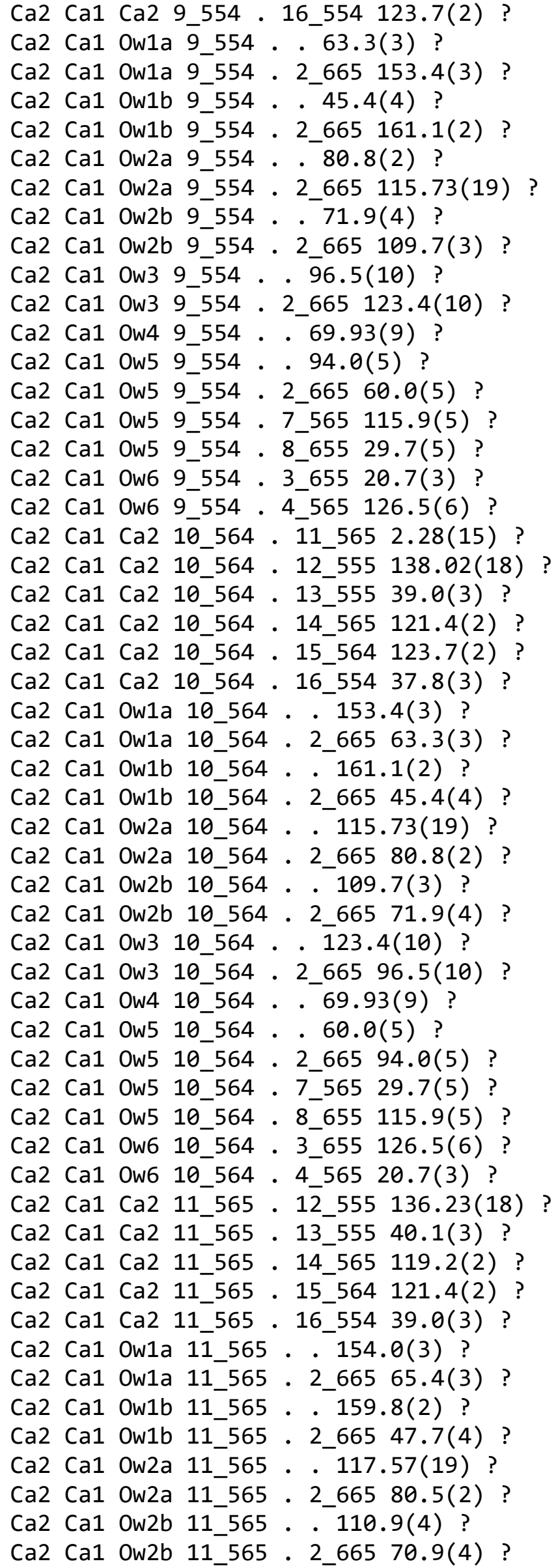




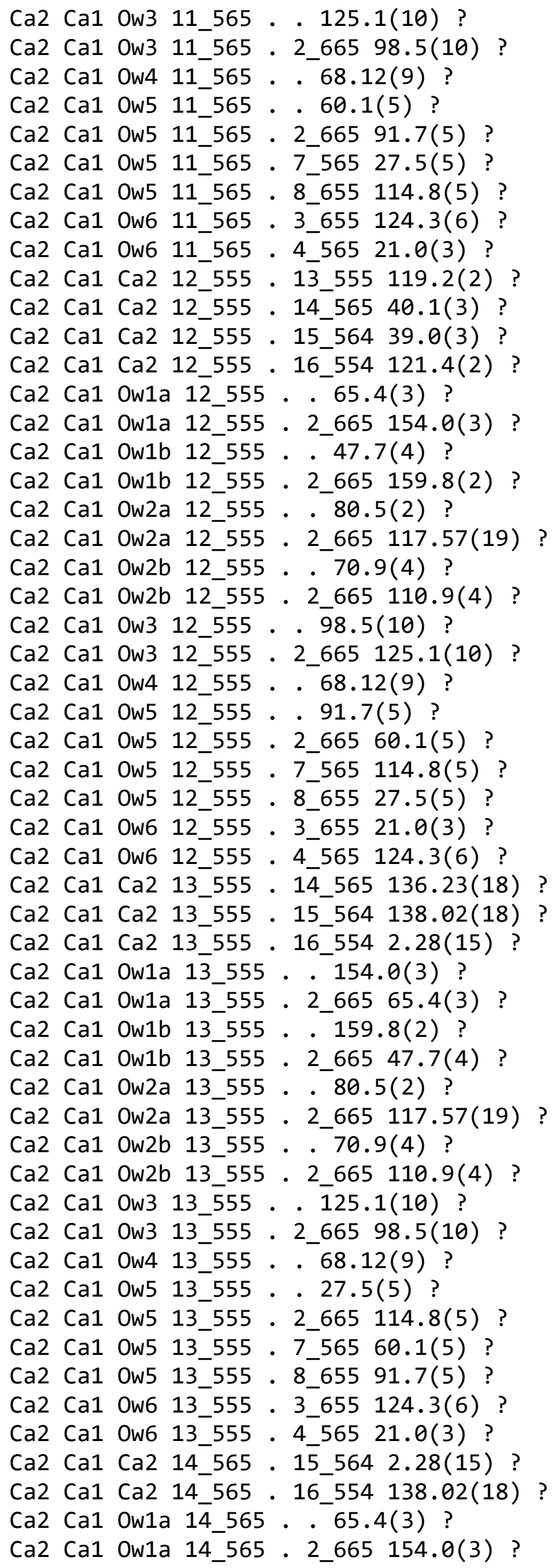




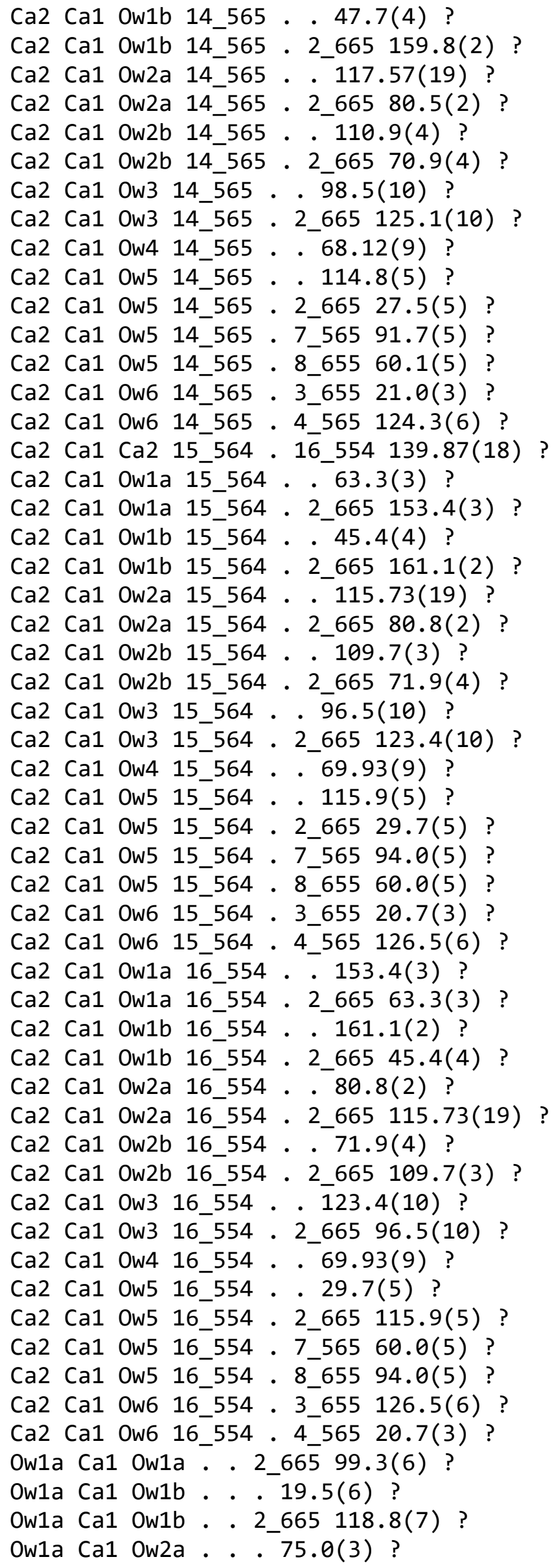




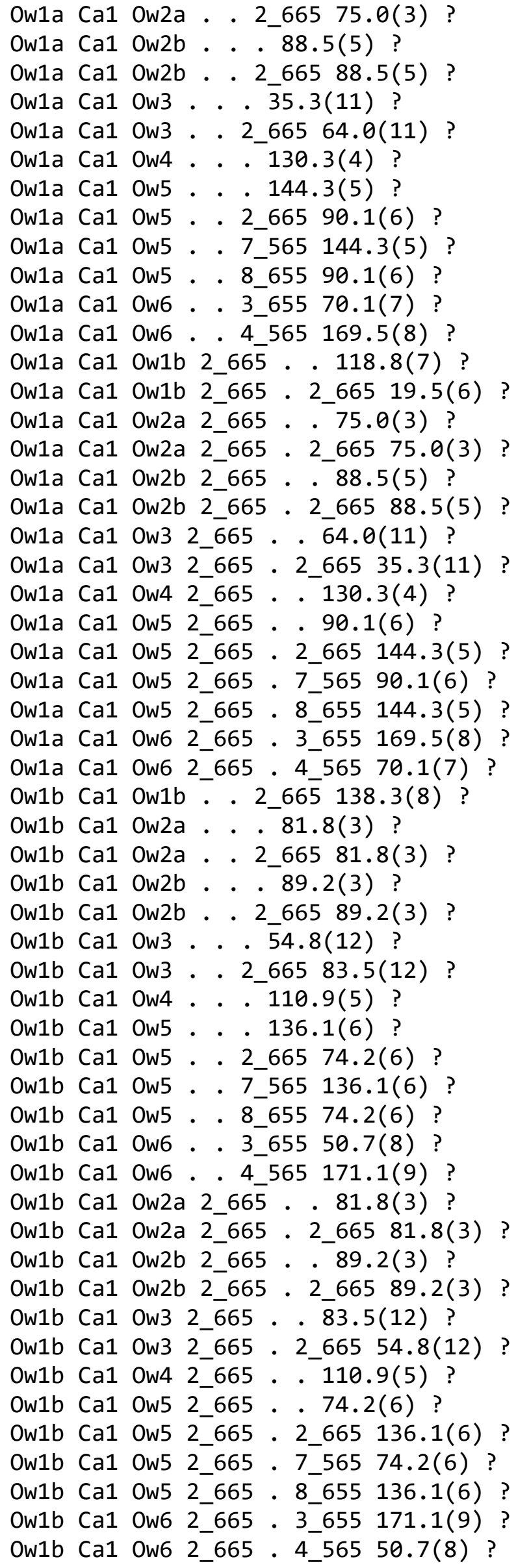




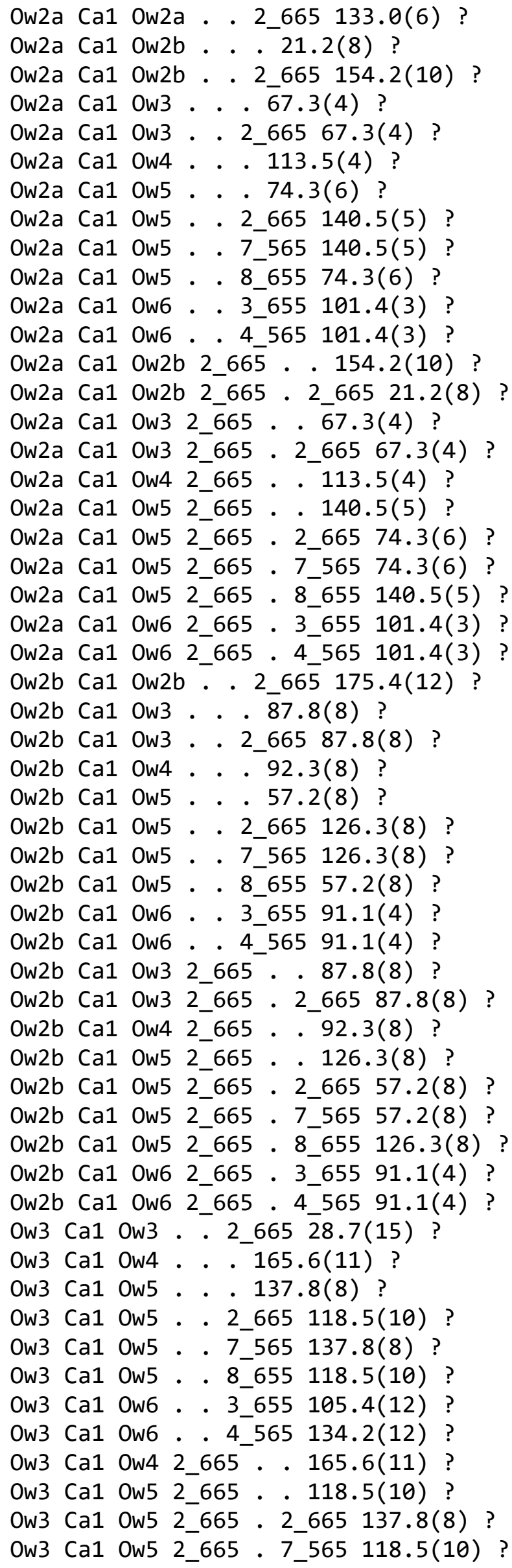




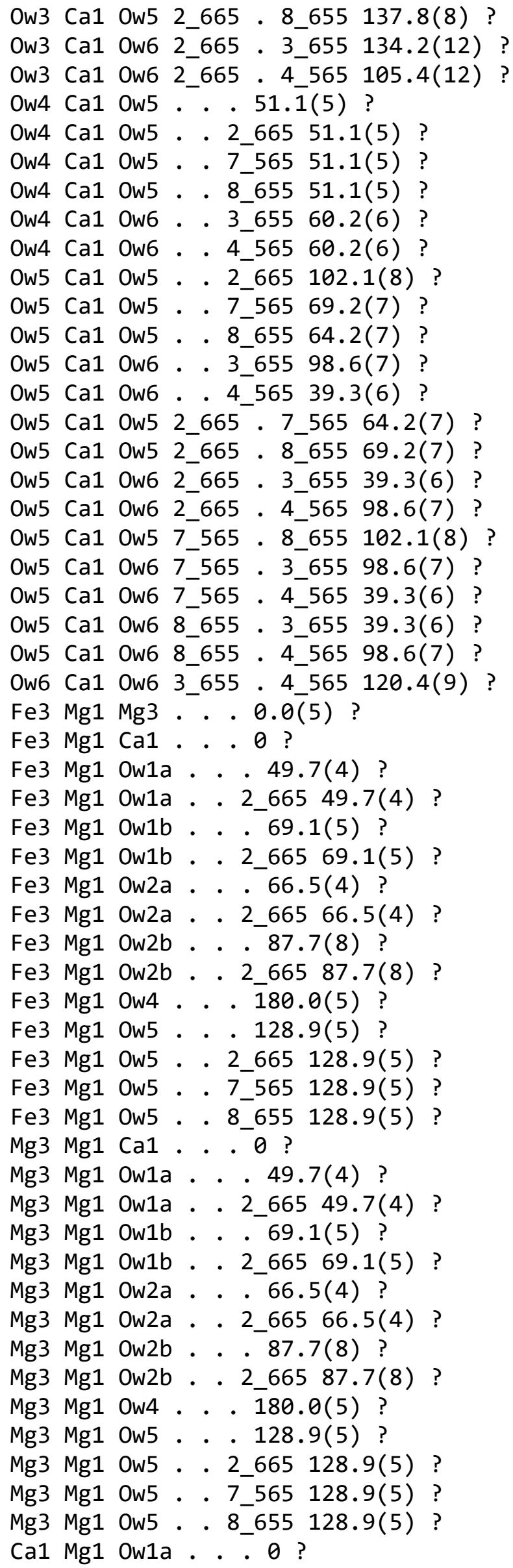




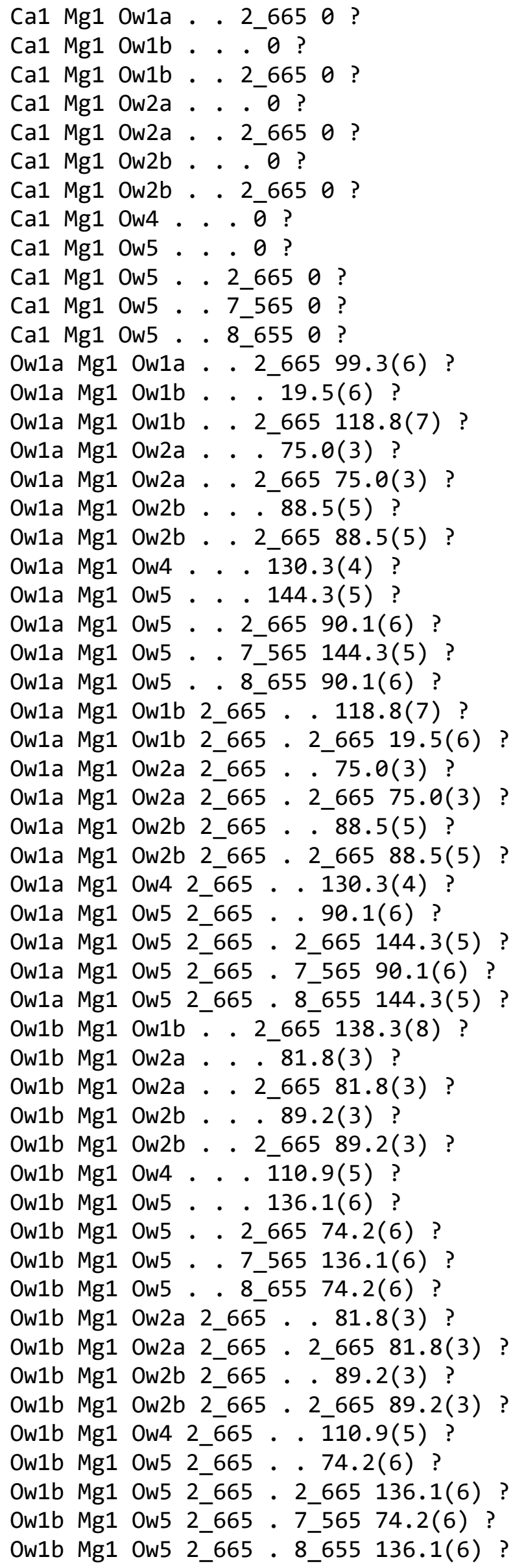




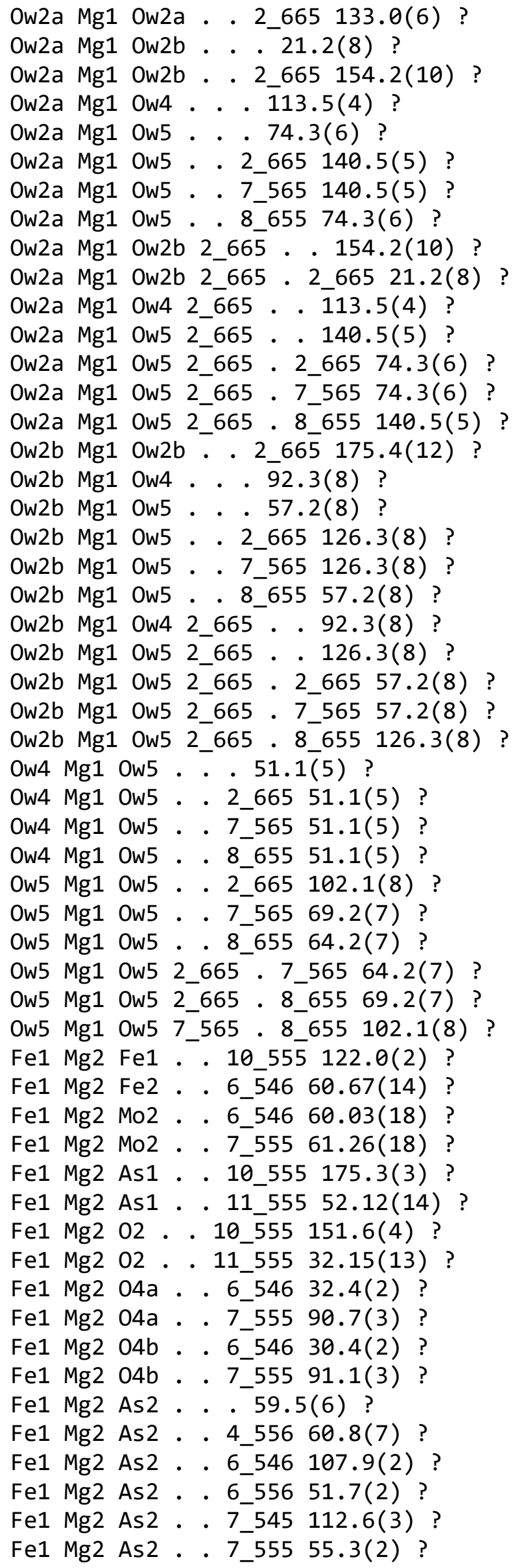




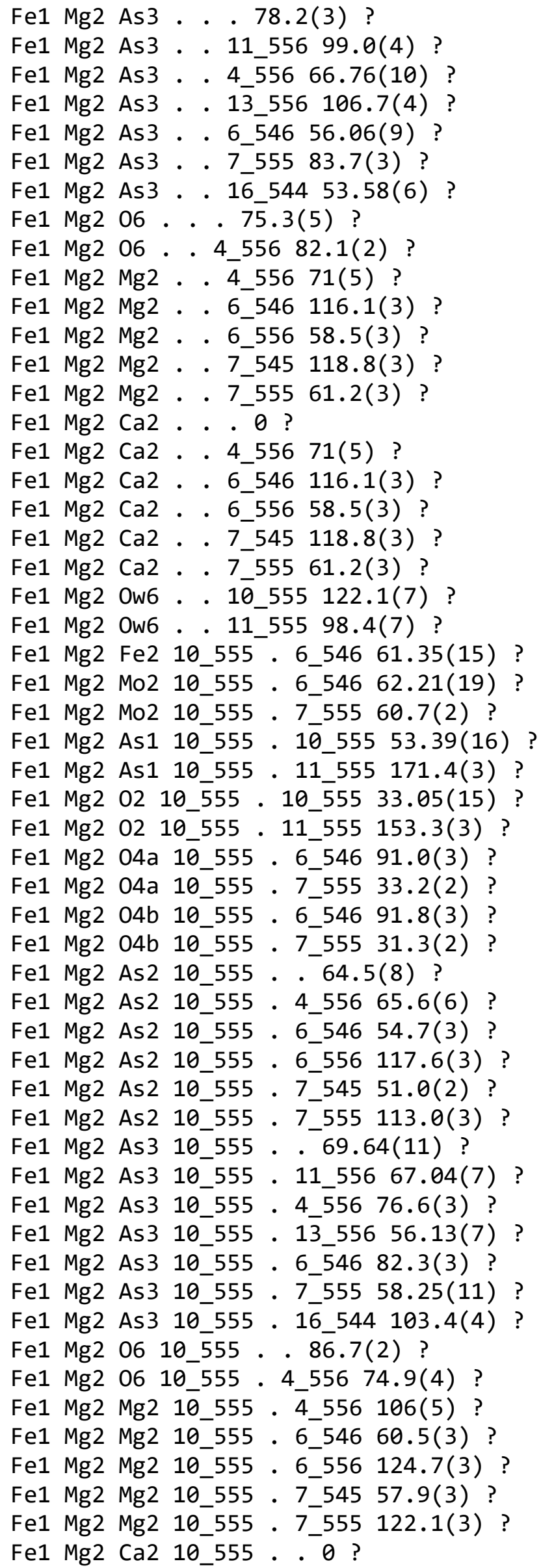




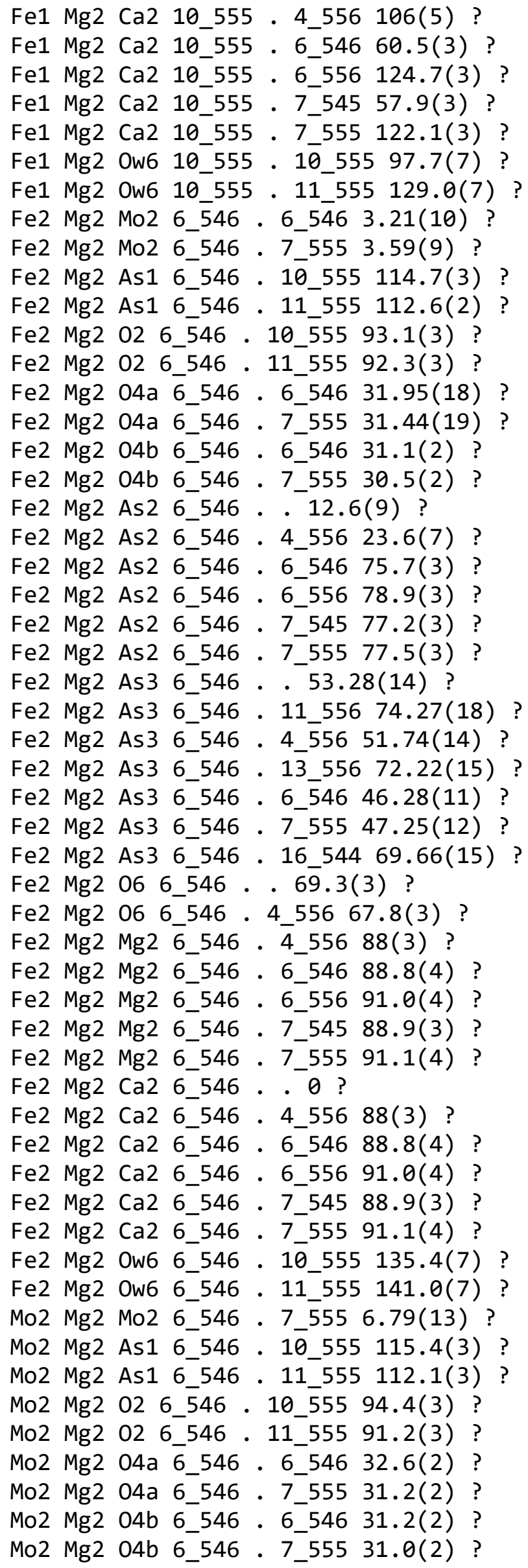




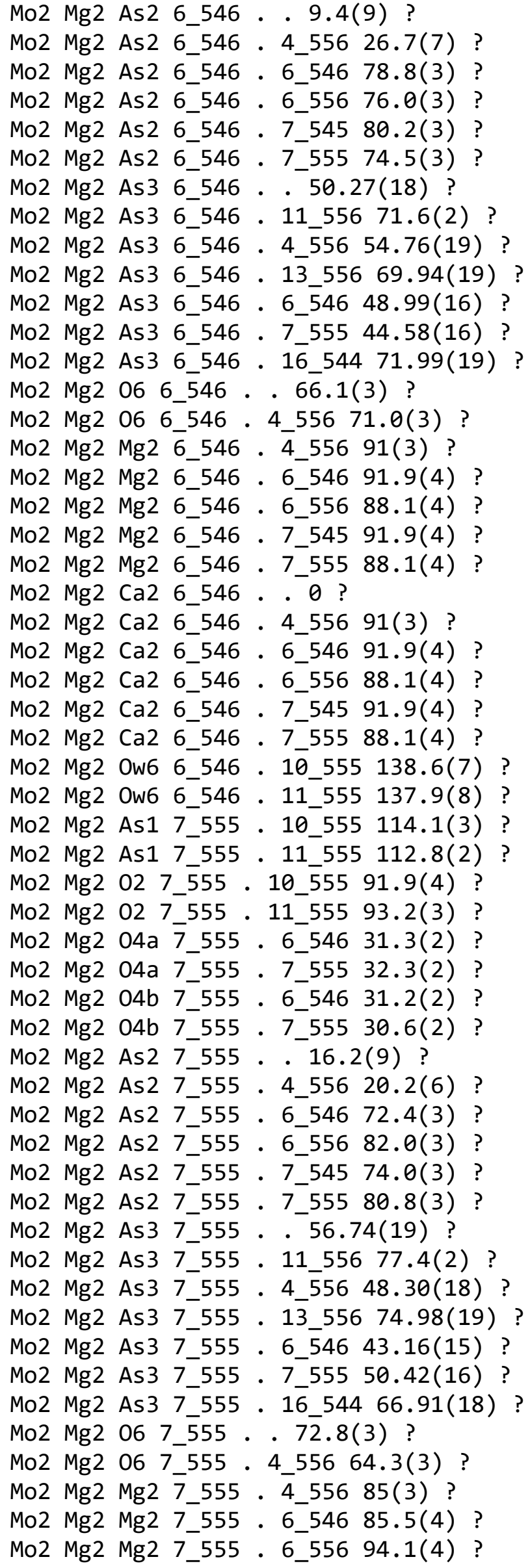




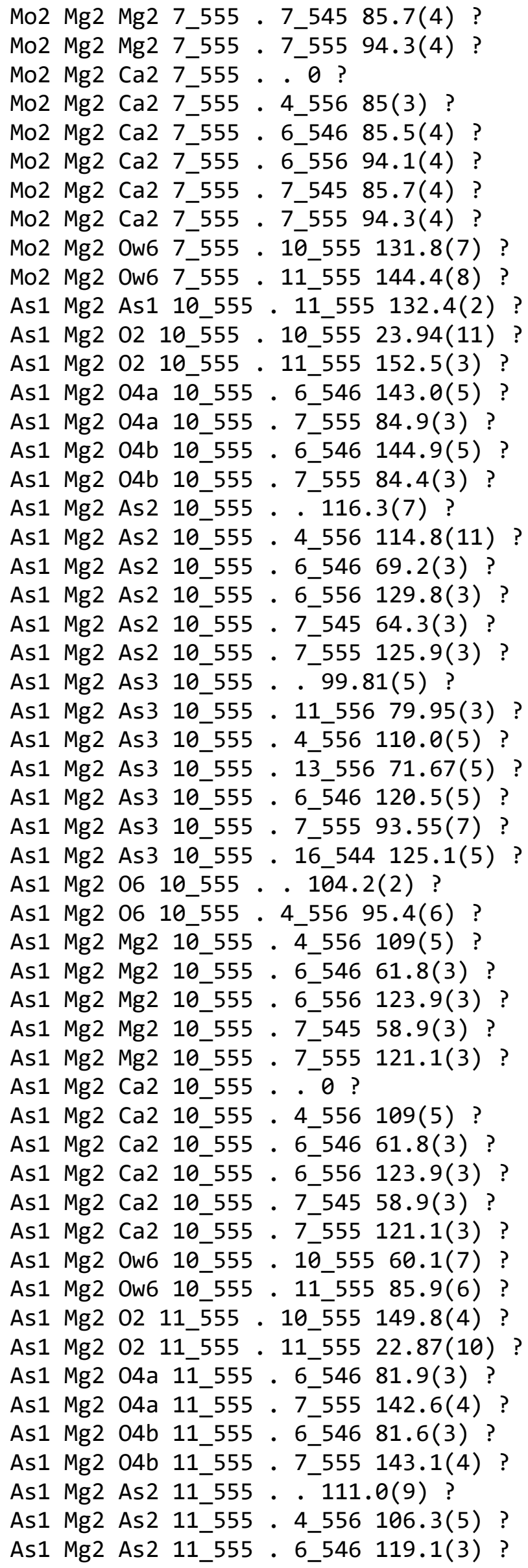




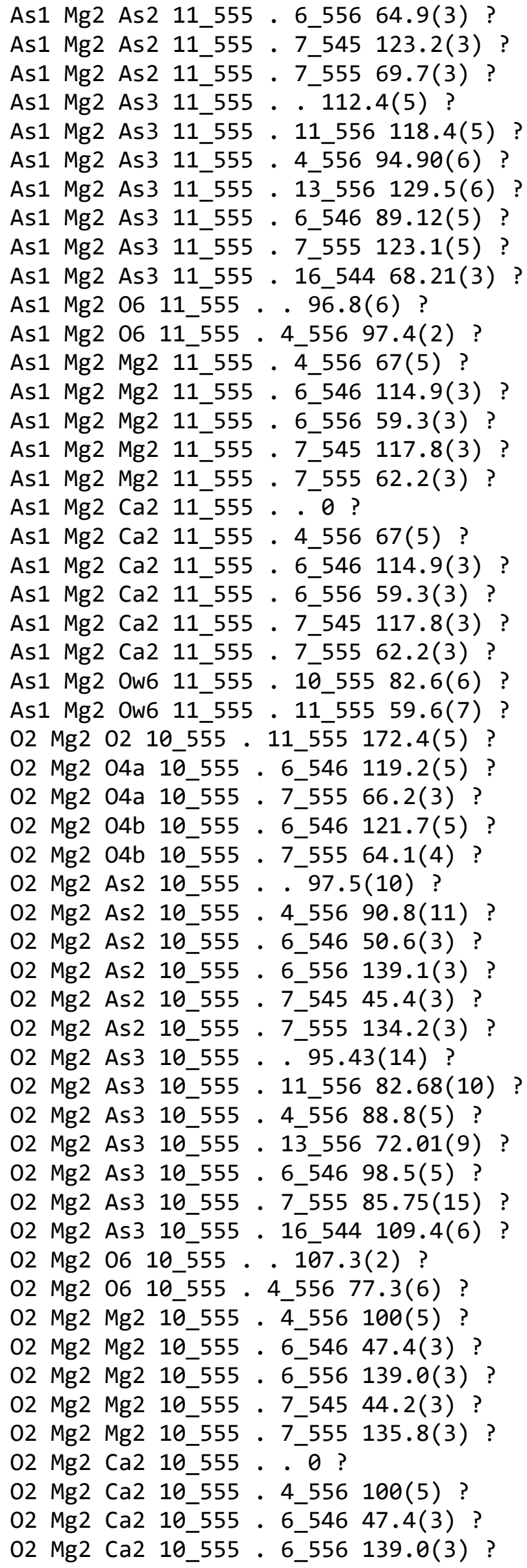




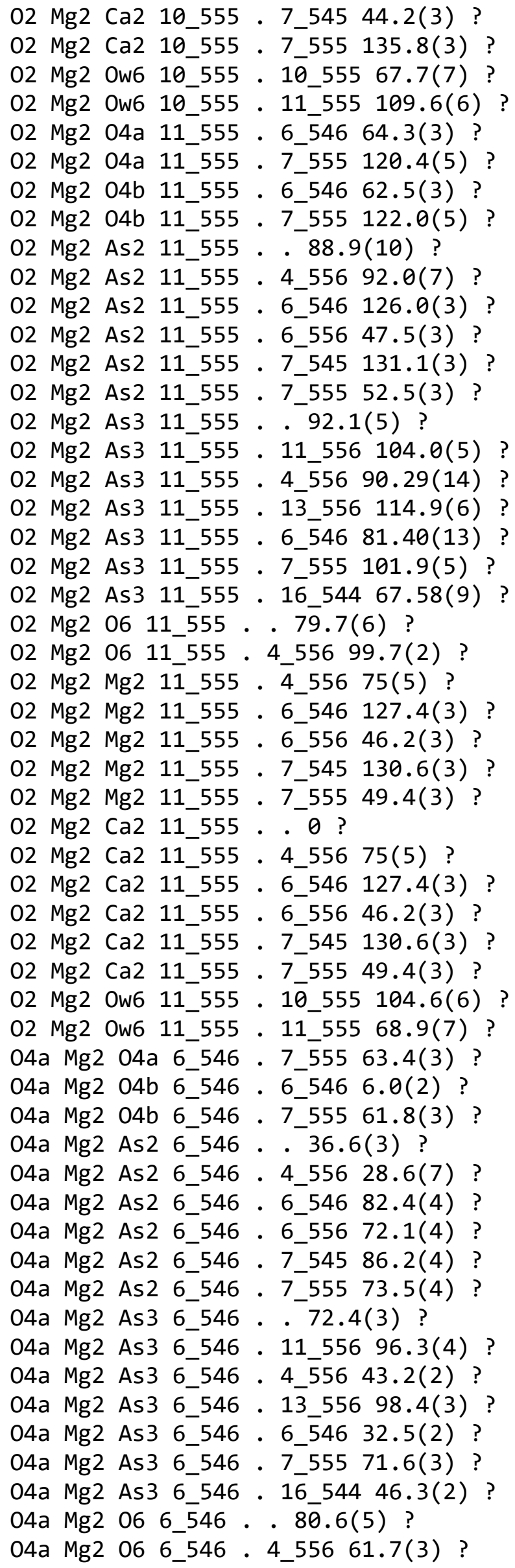




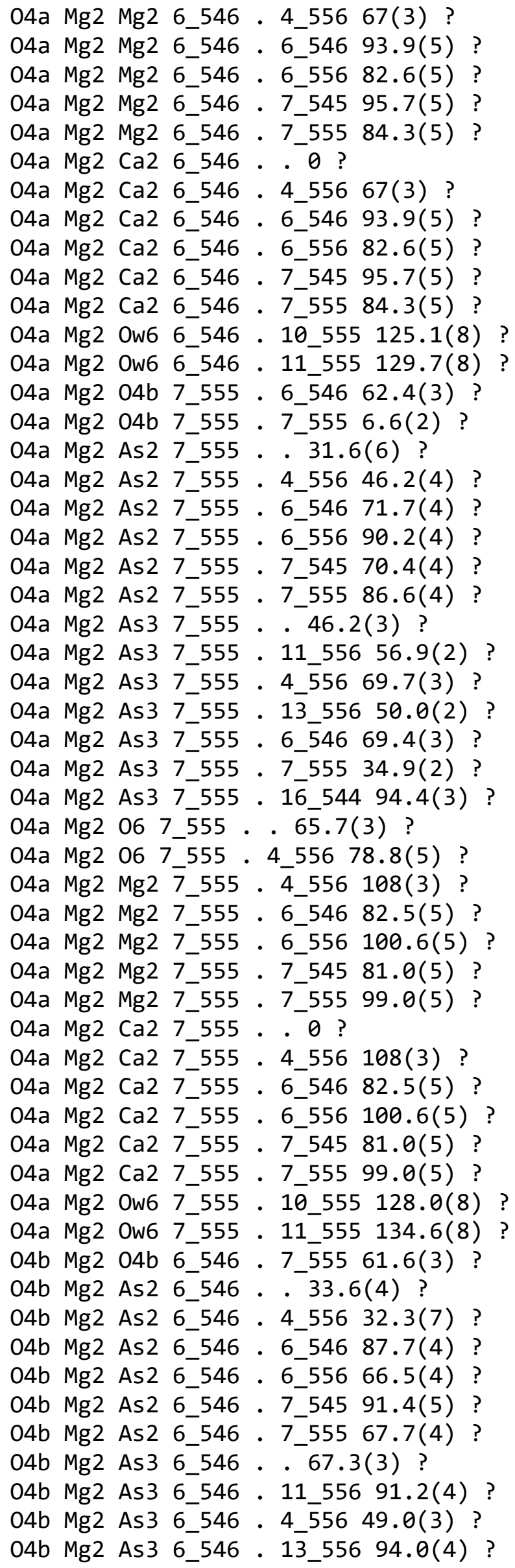




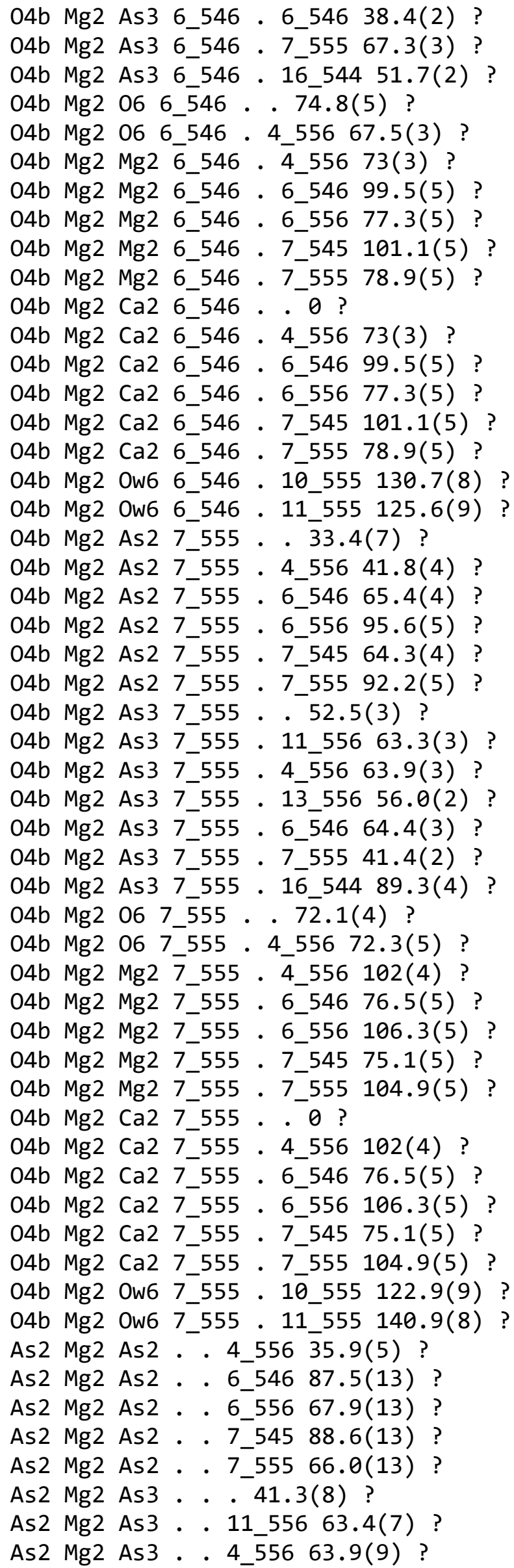




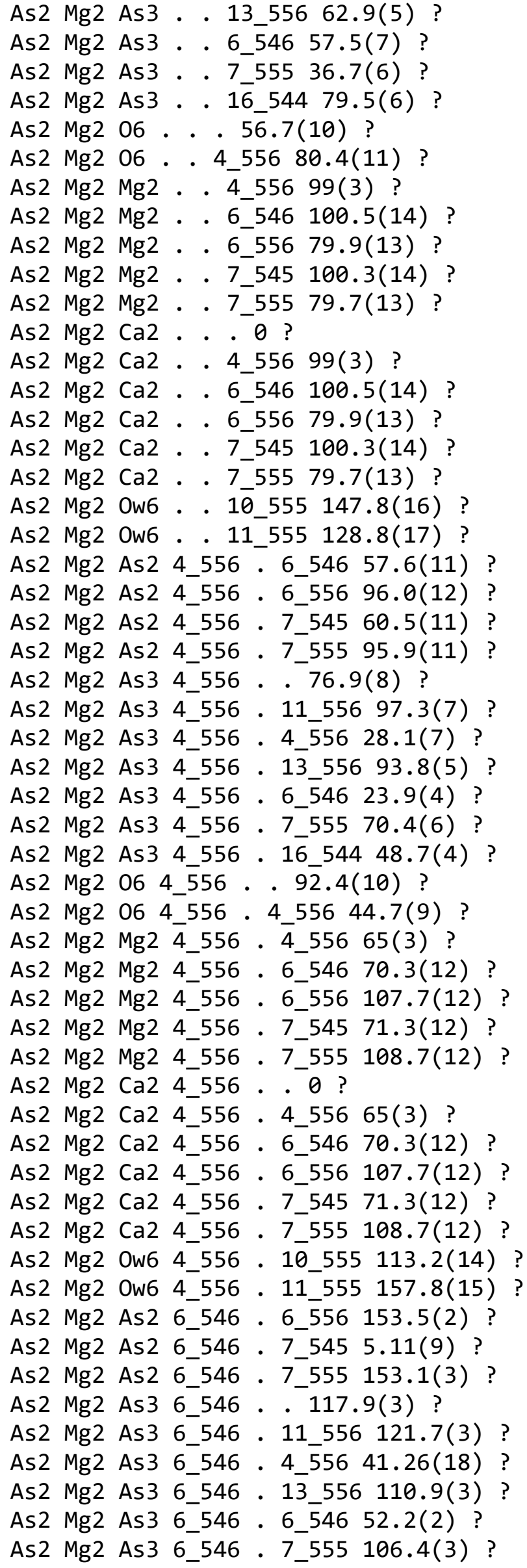




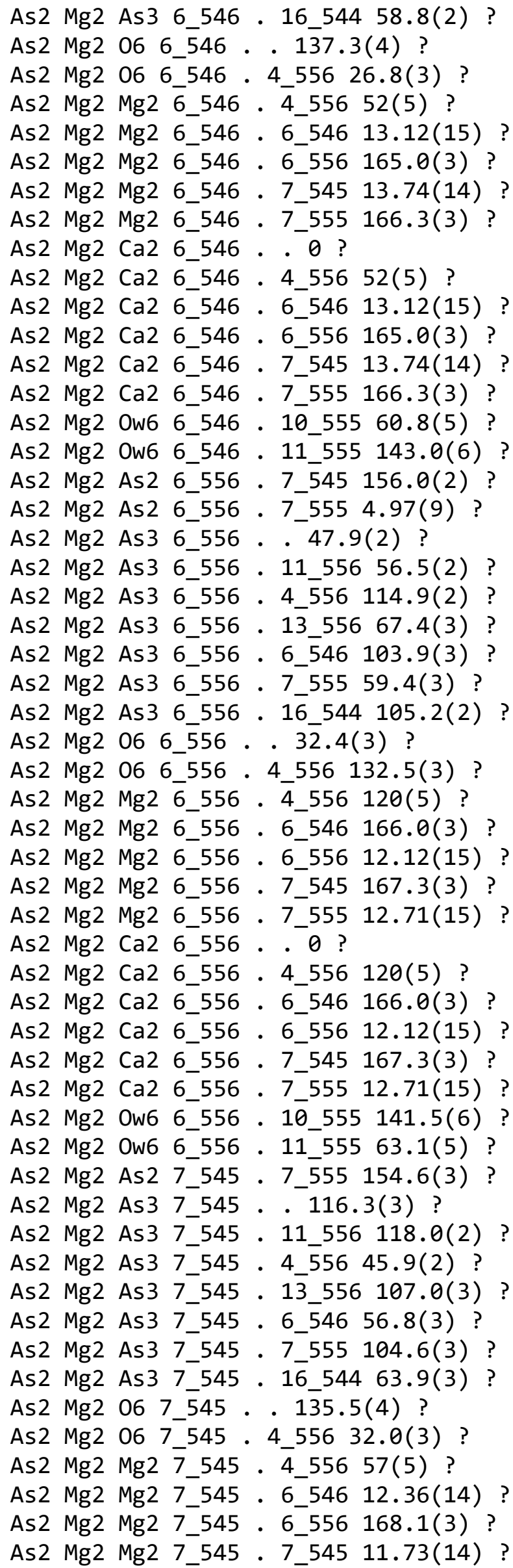


As2 Mg2 Mg2 7_545 . 7_555 168.3(3) ?

As2 Mg2 Ca2 7_545 . . 0 ?

As2 Mg2 Ca2 7_545 . 4_556 57(5) ?

As2 Mg2 Ca2 7_545 . 6_546 12.36(14) ?

As2 Mg2 Ca2 7_545 . 6_556 168.1(3) ?

As2 Mg2 Ca2 7_545 . 7_545 11.73(14) ?

As2 Mg2 Ca2 7_545 . 7_555 168.3(3) ?

As2 Mg2 Ow6 7_545 . 10_555 60.4(5) ?

As2 Mg2 Ow6 7_545 . 11_555 140.9(6) ?

As2 Mg2 As3 7_555 . . 43.4(2) ?

As2 Mg2 As3 7_555 . 11_556 51.6(2) ?

As2 Mg2 As3 7_555 . 4_556 116.6(2) ?

As2 Mg2 As3 7-555 . 13 556 62.4(3) ?

As2 Mg2 As3 7_555 . 6_546 105.7(3) ?

As2 Mg2 As3 7_555 . 7_555 54.9(3) ?

As2 Mg2 As3 7_555 . 16_544 108.8(3) ?

As2 Mg2 06 7_555 . 27.5(3) ?

As2 Mg2 06 7_555 . 4_556 134.7(4) ?

As2 Mg2 Mg2 7_555 . 4_556 124(5) ?

As2 Mg2 Mg2 7_555 . 6_546 166.2(3) ?

As2 Mg2 Mg2 7_555 . 6_556 14.26(15) ?

As2 Mg2 Mg2 7_555 . 7_545 166.3(3) ?

As2 Mg2 Mg2 7_555 . 7_555 13.68(16) ?

As2 Mg2 Ca2 7_555 . . 0 ?

As2 Mg2 Ca2 7_555 . 4_556 124(5) ?

As2 Mg2 Ca2 7_555 . 6_546 166.2(3) ?

As2 Mg2 Ca2 7_555 . 6_556 14.26(15) ?

As2 Mg2 Ca2 7_555 . 7_545 166.3(3) ?

As2 Mg2 Ca2 7_555 . 7_555 13.68(16) ?

As2 Mg2 Ow6 7_555 . 10_555 144.6(6) ?

As2 Mg2 Ow6 7_555 . 11_555 63.9(6) ?

As3 Mg2 As3 . . 11_556 23.94(8) ?

As3 Mg2 As3 . . 4_556 105.0(2) ?

As3 Mg2 As3 . . 13_556 28.68(10) ?

As3 Mg2 As3 . . 6_546 98.7(2) ?

As3 Mg2 As3 . 7 7555 11.76(8) ?

As3 Mg2 As3 . . 16_544 118.6(2) ?

As3 Mg2 06 . . 19.5(2) ?

As3 Mg2 06 . 4 4_556 120.3(4) ?

As3 Mg2 Mg2 . . 4_556 139(3) ?

As3 Mg2 Mg2 . 6 6546 128.0(3) ?

As3 Mg2 Mg2 . . 6_556 56.1(3) ?

As3 Mg2 Mg2 . . 7_545 126.0(3) ?

As3 Mg2 Mg2 . . 7_555 54.0(3) ?

As3 Mg2 Ca2 . . 0 ?

As3 Mg2 Ca2 . . 4_556 139(3) ?

As3 Mg2 Ca2 . 6_546 128.0(3) ?

As3 Mg2 Ca2 . 6_556 56.1(3) ?

As3 Mg2 Ca2 . . 7_545 126.0(3) ?

As3 Mg2 Ca2 . . 7_555 54.0(3) ?

As3 Mg2 Ow6 . . 10_555 159.7(7) ?

As3 Mg2 Ow6 . . 11_555 92.3(7) ?

As3 Mg2 As3 11_556.4_556 124.8(2) ?

As3 Mg2 As3 11_556 . 13_556 11.22(7) ? 
As3 Mg2 As3 11_556 . 6_546 120.6(2) ?

As3 Mg2 As3 11_556 . 7_555 27.03(10) ?

As3 Mg2 As3 11_556 . 16_544 142.3(3) ?

As3 Mg2 06 11_556 . 25.0(2) ?

As3 Mg2 06 11_556 . 4_556 135.7(4) ?

As3 Mg2 Mg2 11_556 . 4_556 162(3) ?

As3 Mg2 Mg2 11_556 . 6_546 126.6(3) ?

As3 Mg2 Mg2 11_556 . 6_556 59.4(3) ?

As3 Mg2 Mg2 11_556 . 7_545 123.6(3) ?

As3 Mg2 Mg2 11_556 . 7_555 56.4(2) ?

As3 Mg2 Ca2 11_556 . . 0 ?

As3 Mg2 Ca2 11_556 . 4_556 162(3) ?

As3 Mg2 Ca2 11_556 . 6_546 126.6(3) ?

As3 Mg2 Ca2 11_556 . 6_556 59.4(3) ?

As3 Mg2 Ca2 11_556 . 7_545 123.6(3) ?

As3 Mg2 Ca2 11_556 . 7_555 56.4(2) ?

As3 Mg2 Ow6 11_556 . 10_555 136.8(7) ?

As3 Mg2 Ow6 11_556 . 11_555 77.7(7) ?

As3 Mg2 As3 4_556 . 13_556 119.6(2) ?

As3 Mg2 As3 4_556 . 6_546 11.06(7) ?

As3 Mg2 As3 4_556 . 7_555 98.2(2) ?

As3 Mg2 As3 4_556 . 16_544 27.09(9) ?

As3 Mg2 06 4_556 . . 119.7(4) ?

As3 Mg2 06 4_556 . 4_556 18.67(19) ?

As3 Mg2 Mg2 4_556 . 4_556 38(3) ?

As3 Mg2 Mg2 4_556 . 6_546 51.3(2) ?

As3 Mg2 Mg2 4_556 . 6_556 124.3(3) ?

As3 Mg2 Mg2 4_556 . 7_545 53.5(3) ?

As3 Mg2 Mg2 4_556 . 7_555 126.5(3) ?

As3 Mg2 Ca2 4_556 . . 0 ?

As3 Mg2 Ca2 4_556 . 4_556 38(3) ?

As3 Mg2 Ca2 4_556 . 6_546 51.3(2) ?

As3 Mg2 Ca2 4_556 . 6_556 124.3(3) ?

As3 Mg2 Ca2 4_556 . 7_545 53.5(3) ?

As3 Mg2 Ca2 4_556 . 7_555 126.5(3) ?

As3 Mg2 Ow6 4_556 . 10_555 86.6(7) ?

As3 Mg2 Ow6 4_556 . 11_555 153.5(7) ?

As3 Mg2 As3 13_556 . 6_546 117.6(2) ?

As3 Mg2 As3 13_556 . 7_555 26.75(8) ?

As3 Mg2 As3 13_556 . 16_544 141.9(2) ?

As3 Mg2 06 13_556 . 35.3(2) ?

As3 Mg2 06 13_556 . 4_556 127.4(4) ?

As3 Mg2 Mg2 13_556 . 4_556 158(4) ?

As3 Mg2 Mg2 13_556 • 6_546 115.4(3) ?

As3 Mg2 Mg2 13_556 . 6_556 70.6(3) ?

As3 Mg2 Mg2 13_556 . 7_545 112.4(3) ?

As3 Mg2 Mg2 13_556 . 7_555 67.6(3) ?

As3 Mg2 Ca2 13_556. . 0 ?

As3 Mg2 Ca2 13_556 . 4_556 158(4) ?

As3 Mg2 Ca2 13_556 . 6_546 115.4(3) ?

As3 Mg2 Ca2 13_556 . 6_556 70.6(3) ?

As3 Mg2 Ca2 13_556 . 7_545 112.4(3) ?

As3 Mg2 Ca2 13_556 . 7_555 67.6(3) ?

As3 Mg2 Ow6 13_556 . 10_555 131.0(7) ? 
As3 Mg2 Ow6 13_556 . 11_555 85.0(7) ?

As3 Mg2 As3 6_546 . 7_555 93.5(2) ?

As3 Mg2 As3 6_546 . 16_544 25.05(7) ?

As3 Mg2 06 6_546 . . 111.3(4) ?

As3 Mg2 06 6_546 . 4_556 29.16(19) ?

As3 Mg2 Mg2 6_546 . 4_556 42(3) ?

As3 Mg2 Mg2 6_546 . 6_546 62.4(3) ?

As3 Mg2 Mg2 6_546 . 6_556 113.3(3) ?

As3 Mg2 Mg2 6_546 . 7_545 64.5(3) ?

As3 Mg2 Mg2 6_546 . 7_555 115.5(3) ?

As3 Mg2 Ca2 6_546 . . 0 ?

As3 Mg2 Ca2 6_546 . 4_556 42(3) ?

As3 Mg2 Ca2 6_546 . 6_546 62.4(3) ?

As3 Mg2 Ca2 6_546 . 6_556 113.3(3) ?

As3 Mg2 Ca2 6_546 . 7_545 64.5(3) ?

As3 Mg2 Ca2 6_546 . 7_555 115.5(3) ?

As3 Mg2 Ow6 6_546 . 10_555 95.2(7) ?

As3 Mg2 Ow6 6_546 . 11_555 148.7(7) ?

As3 Mg2 As3 7_555 . 16_544 116.1(2) ?

As3 Mg2 06 7_555 . 31.0(2) ?

As3 Mg2 06 7_555 . 4_556 111.5(4) ?

As3 Mg2 Mg2 7_555 . 4_556 135(3) ?

As3 Mg2 Mg2 7_555 . 6_546 116.3(4) ?

As3 Mg2 Mg2 7_555 . 6_556 67.8(3) ?

As3 Mg2 Mg2 7_555 . 7_545 114.3(4) ?

As3 Mg2 Mg2 7_555 . 7_555 65.7(3) ?

As3 Mg2 Ca2 7_555 . . 0 ?

As3 Mg2 Ca2 7_555 . 4_556 135(3) ?

As3 Mg2 Ca2 7_555 . 6_546 116.3(4) ?

As3 Mg2 Ca2 7_555 . 6_556 67.8(3) ?

As3 Mg2 Ca2 7_555 . 7_545 114.3(4) ?

As3 Mg2 Ca2 7_555 . 7_555 65.7(3) ?

As3 Mg2 Ow6 7_555 . 10_555 153.0(7) ?

As3 Mg2 Ow6 7_555 . 11_555 101.9(7) ?

As3 Mg2 06 16_544 . . 125.4(4) ?

As3 Mg2 06 16_544 . 4_556 32.2(2) ?

As3 Mg2 Mg2 16_544 . 4 _556 21(3) ?

As3 Mg2 Mg2 16_544 . 6_546 63.7(3) ?

As3 Mg2 Mg2 16_544 . 6_556 110.2(3) ?

As3 Mg2 Mg2 16_544 . 7_545 66.7(3) ?

As3 Mg2 Mg2 16_544 . 7_555 113.3(3) ?

As3 Mg2 Ca2 16_544 . . 0 ?

As3 Mg2 Ca2 16_544 . 4_556 21(3) ?

As3 Mg2 Ca2 16_544 . 6_546 63.7(3) ?

As3 Mg2 Ca2 16_544 . 6_556 110.2(3) ?

As3 Mg2 Ca2 16_544 . 7_545 66.7(3) ?

As3 Mg2 Ca2 16_544 . 7_555 113.3(3) ?

As3 Mg2 Ow6 16_544 . 10_555 79.1(7) ?

As3 Mg2 Ow6 16_544. 11_555 126.5(7) ?

06 Mg2 06.44556 137.0(5) ?

06 Mg2 Mg2 . . 4_556 145(4) ?

06 Mg2 Mg2 . . 6_546 146.8(4) ?

06 Mg2 Mg2 . 6_556 38.1(4) ?

06 Mg2 Mg2 . 7 7545 144.4(4) ? 


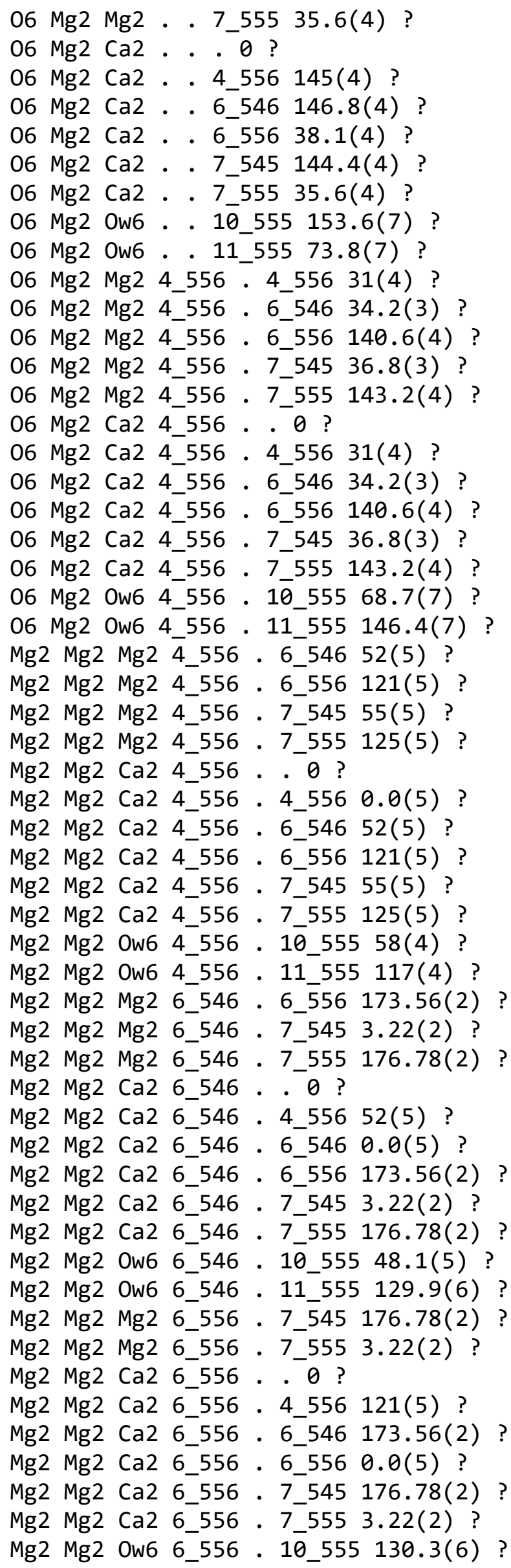




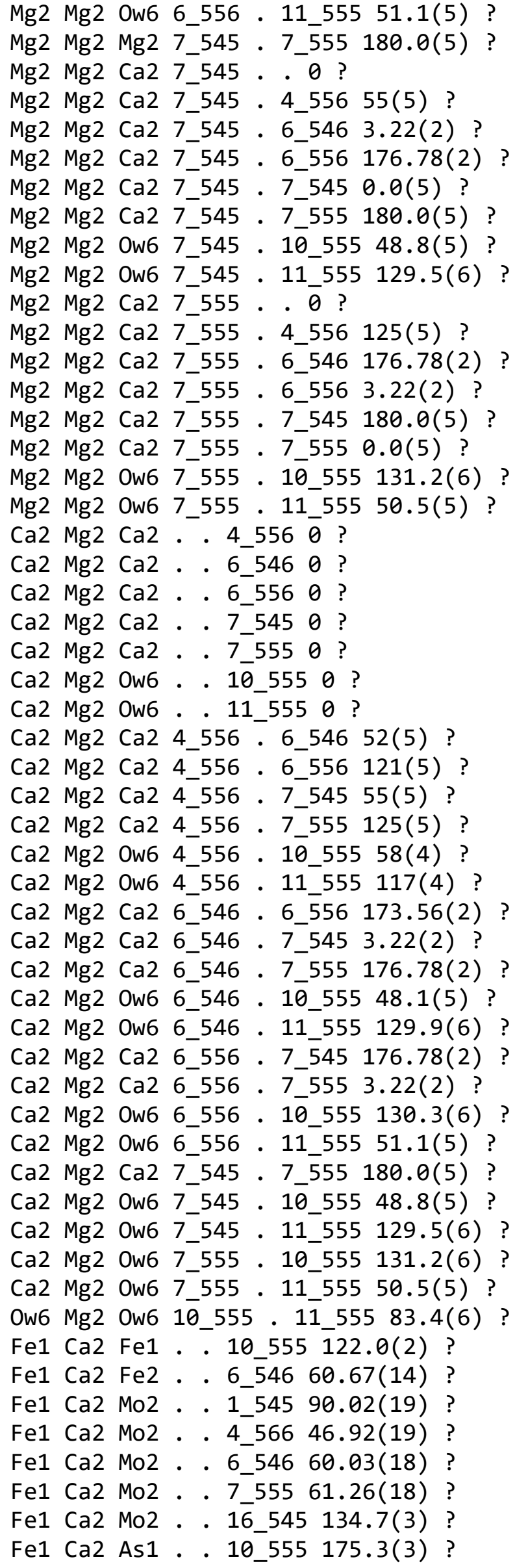




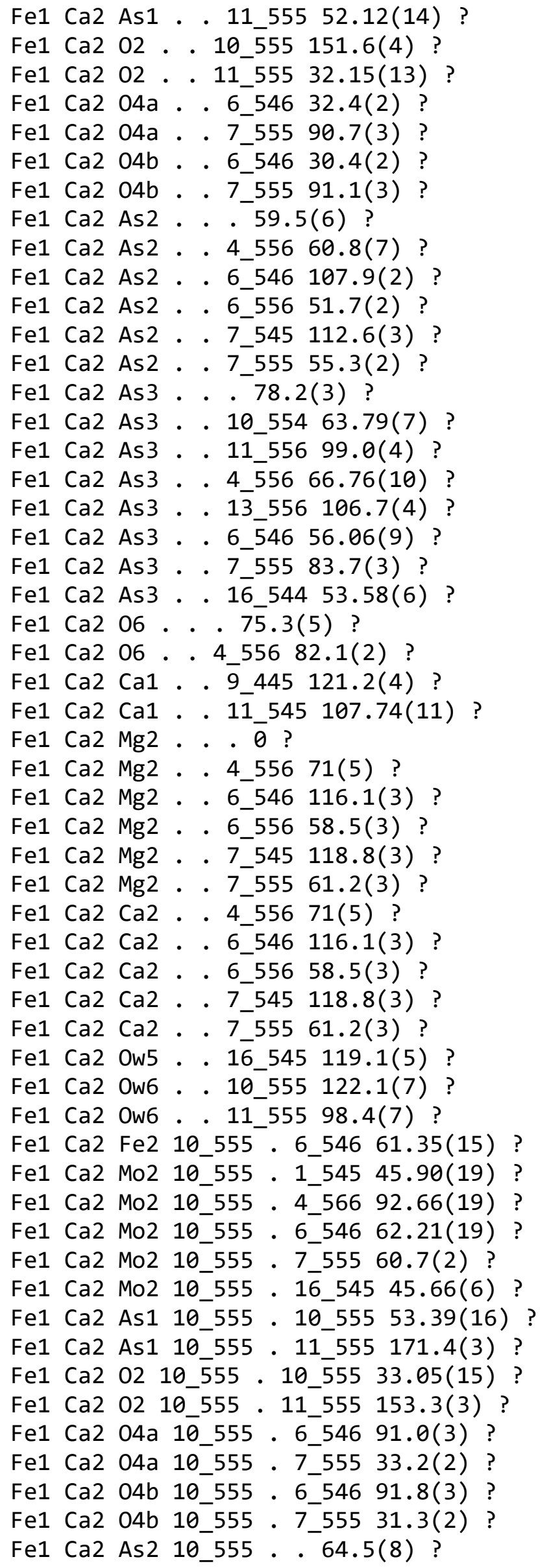




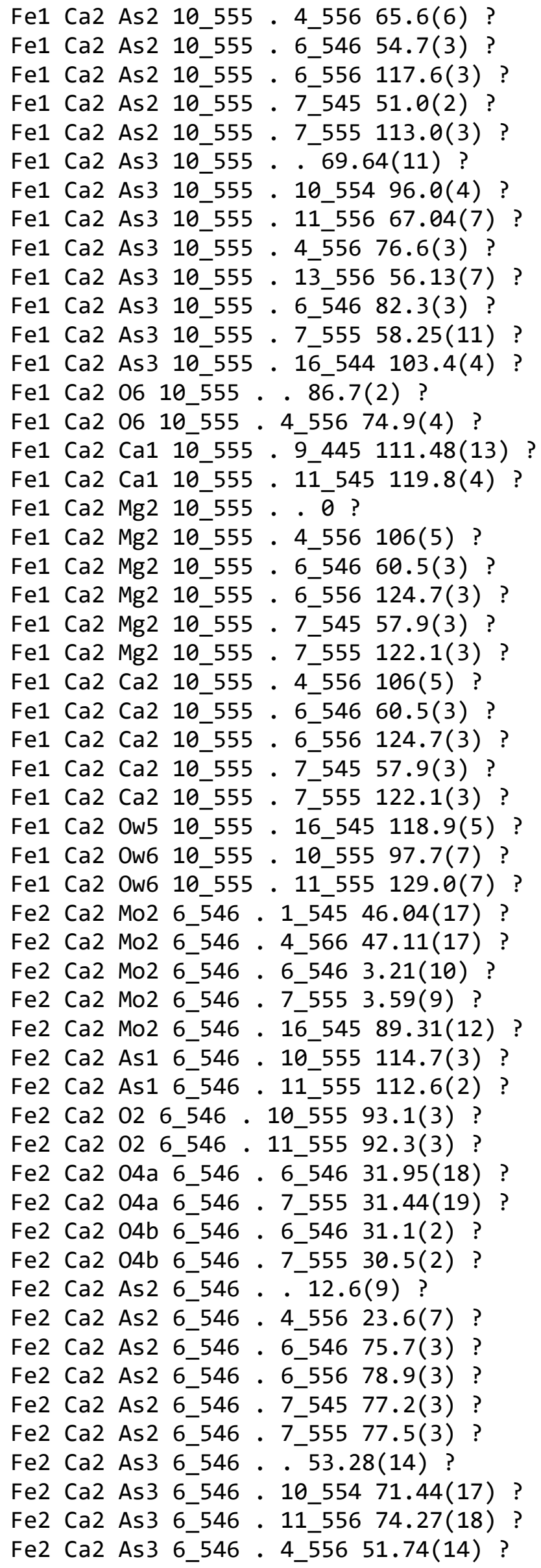




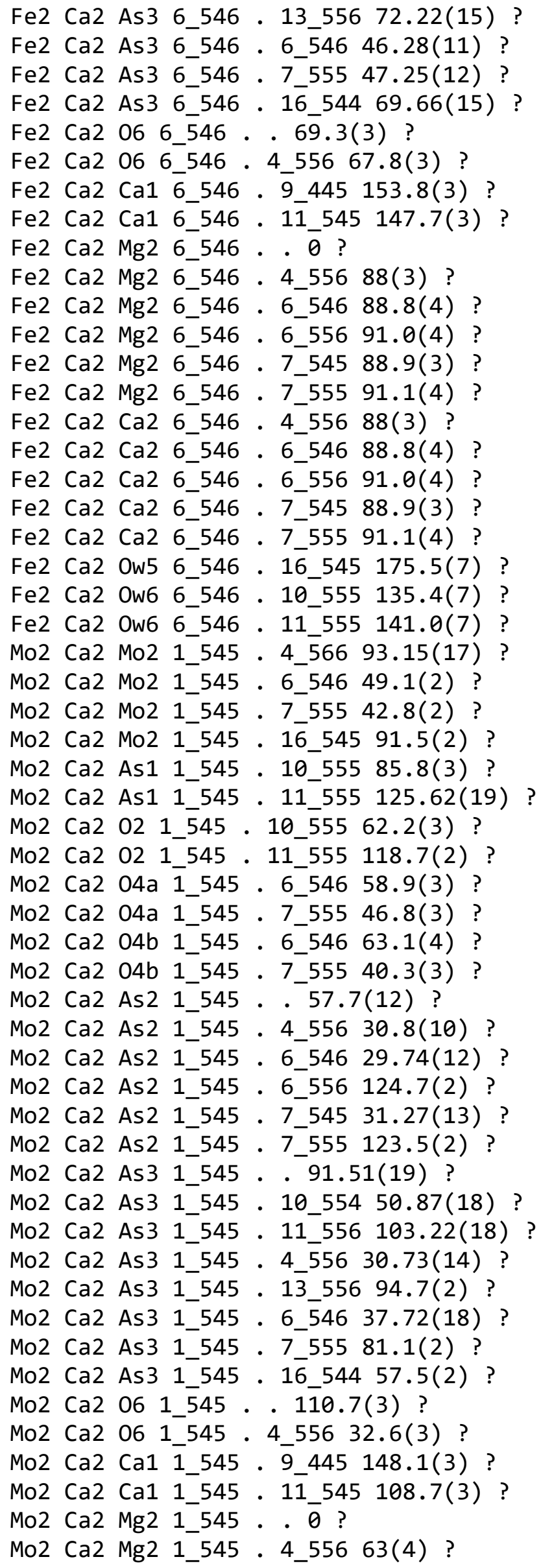




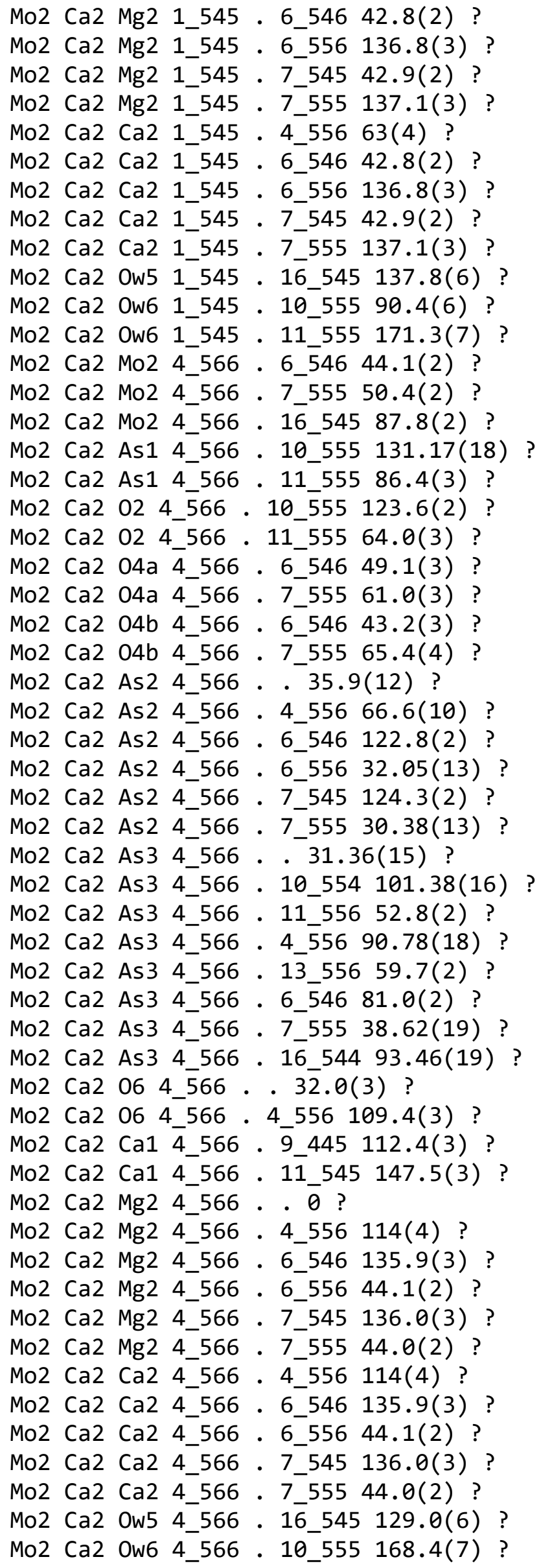




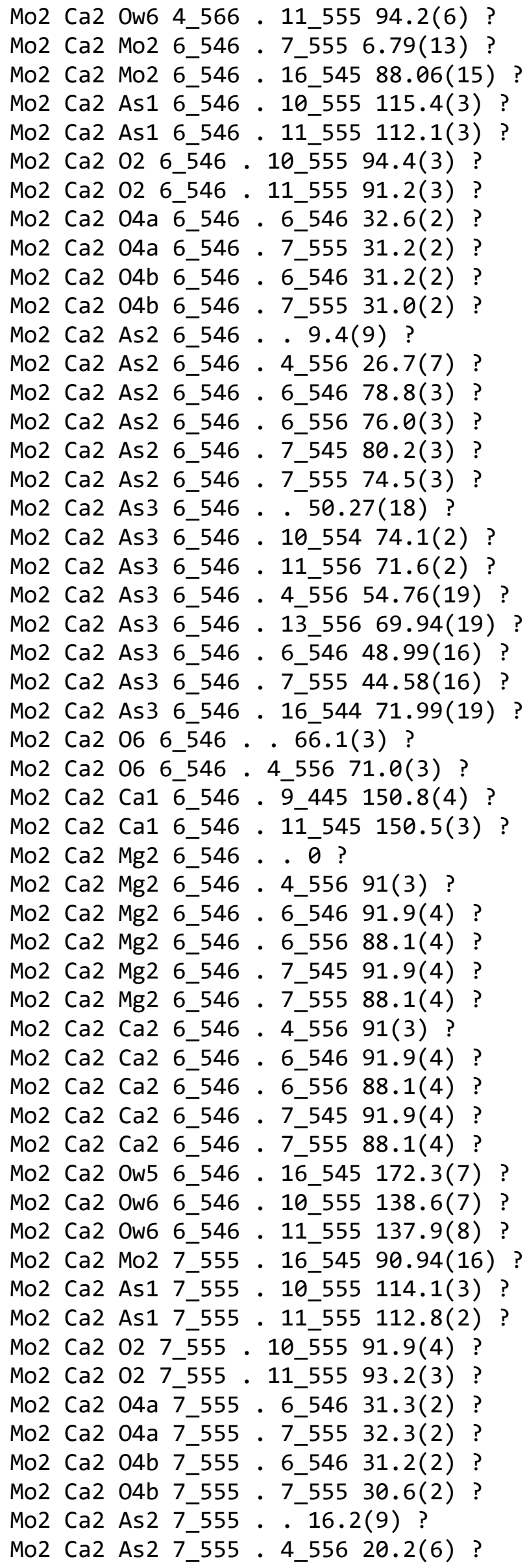




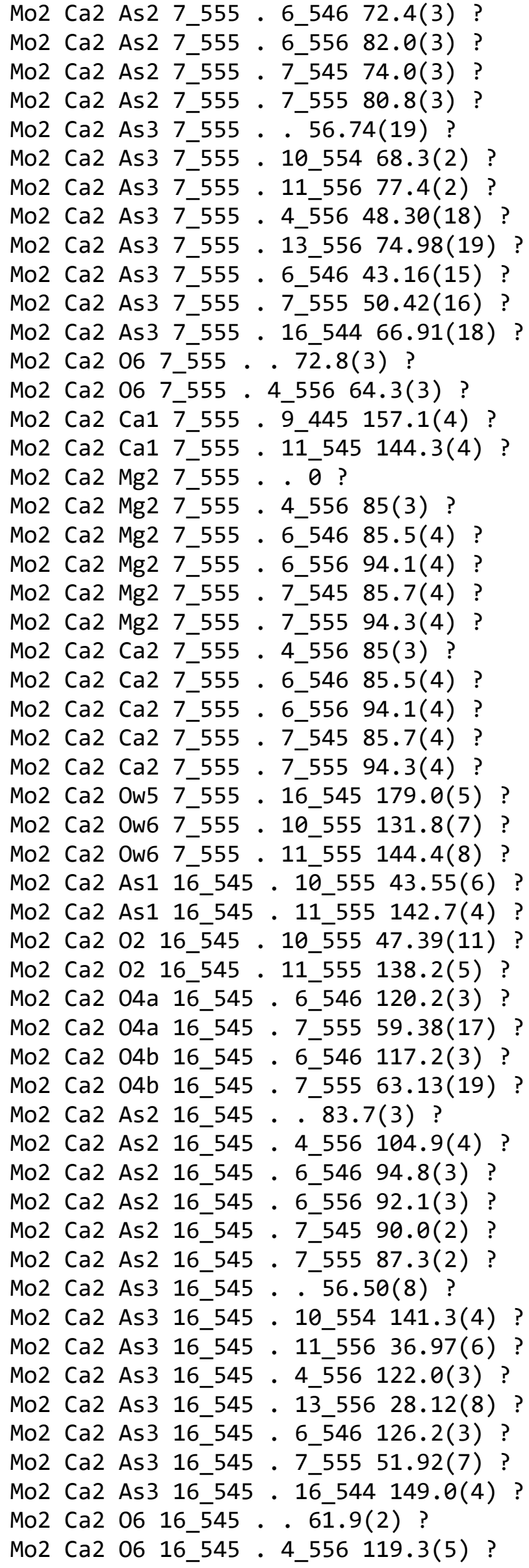




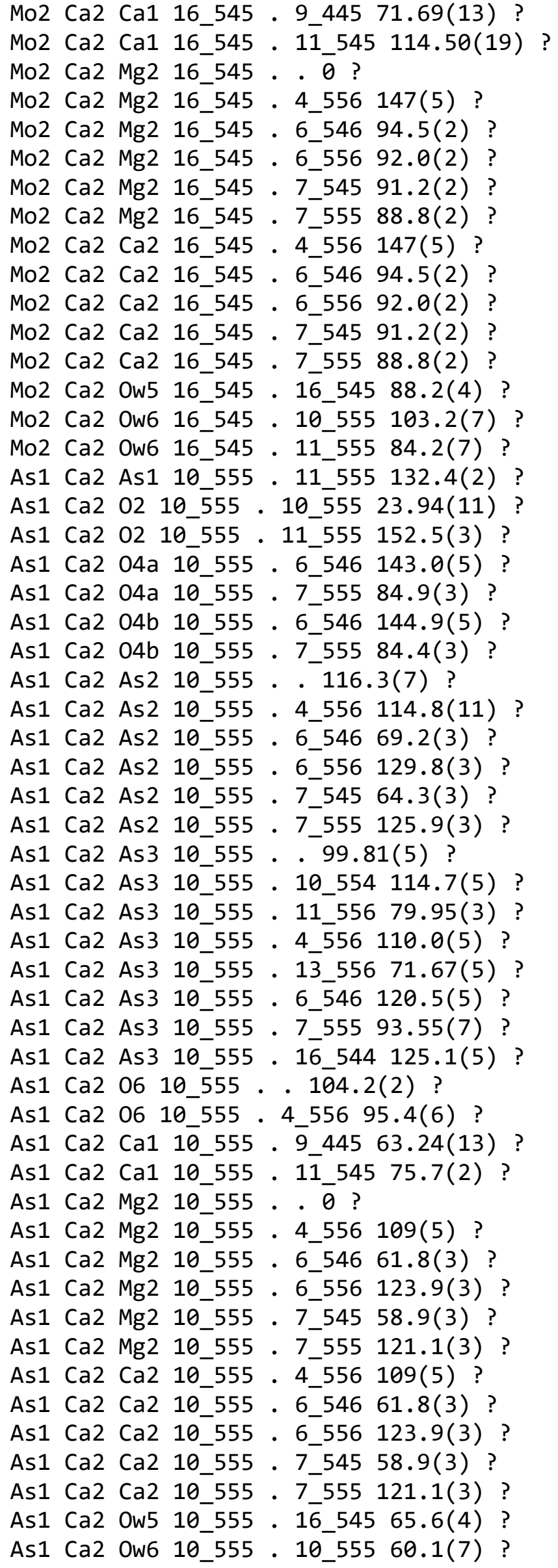




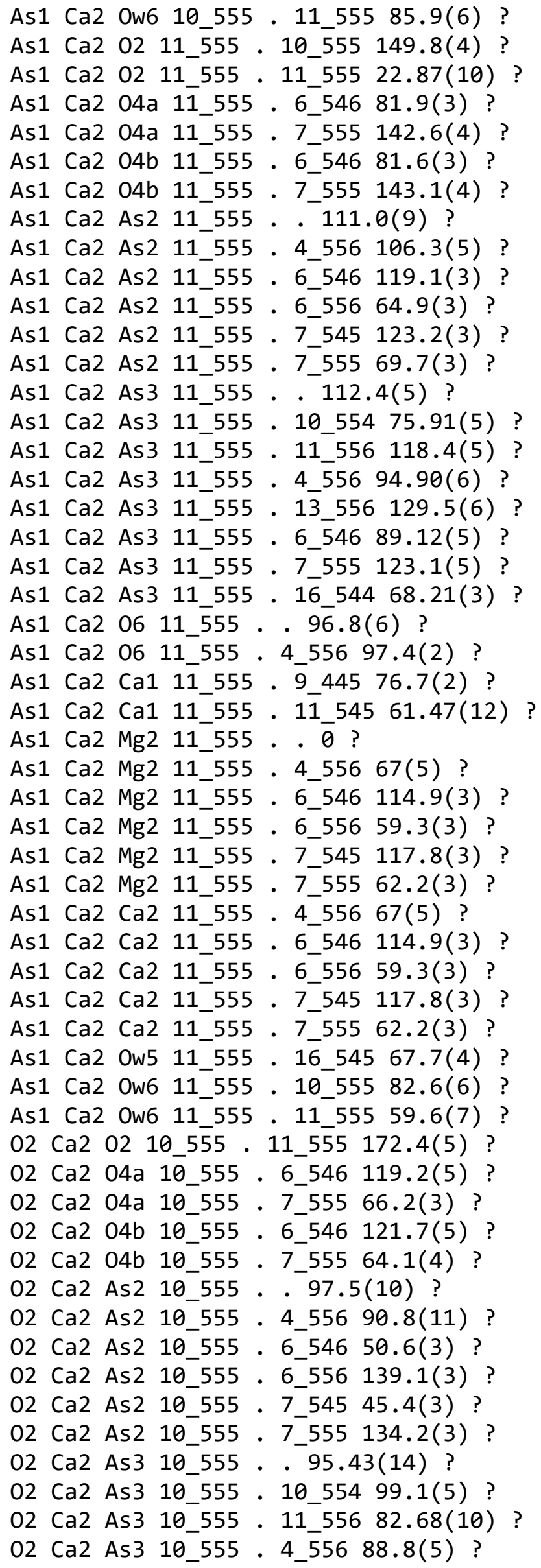


02 Ca2 As3 10_555 . 13_556 72.01(9) ?

02 Ca2 As3 10_555 . 6_546 98.5(5) ?

02 Ca2 As3 10_555 . 7_555 85.75(15) ?

02 Ca2 As3 10_555 . 16_544 109.4(6) ?

02 Ca2 06 10_555 . 107.3(2) ?

02 Ca2 06 10_555 . 4_556 77.3(6) ?

02 Ca2 Ca1 10_555 . 9_445 87.17(19) ?

02 Ca2 Ca1 10_555 . 11_545 88.4(3) ?

02 Ca2 Mg2 10_555.. 0 ?

02 Ca2 Mg2 10_555 . 4_556 100(5) ?

02 Ca2 Mg2 10_555 . 6_546 47.4(3) ?

02 Ca2 Mg2 10_555 . 6_556 139.0(3) ?

02 Ca2 Mg2 10_555.7_545 44.2(3) ?

02 Ca2 Mg2 10_555 . 7_555 135.8(3) ?

02 Ca2 Ca2 10_555 . 4_556 100(5) ?

02 Ca2 Ca2 10_555 . 6_546 47.4(3) ?

02 Ca2 Ca2 10_555 . 6_556 139.0(3) ?

$02 \mathrm{Ca} 2 \mathrm{Ca} 2$ 10_555 . 7_545 44.2(3) ?

02 Ca2 Ca2 10_555 . 7_555 135.8(3) ?

02 Ca2 Ow5 10_555 . 16_545 87.9(5) ?

02 Ca2 Ow6 10_555 . 10_555 67.7(7) ?

02 Ca2 Ow6 10_555 . 11_555 109.6(6) ?

02 Ca2 04a 11_555 . 6_546 64.3(3) ?

02 Ca2 04a 11_555 . 7_555 120.4(5) ?

02 Ca2 04b 11_555 . 6_546 62.5(3) ?

02 Ca2 04b 11_555 . 7_555 122.0(5) ?

02 Ca2 As2 11_555 . 88.9(10) ?

02 Ca2 As2 11_555 . 4_556 92.0(7) ?

02 Ca2 As2 11_555 . 6_546 126.0(3) ?

02 Ca2 As2 11_555 . 6_556 47.5(3) ?

02 Ca2 As2 11_555 . 7_545 131.1(3) ?

02 Ca2 As2 11_555 . 7_555 52.5(3) ?

02 Ca2 As3 11_555. . 92.1(5) ?

02 Ca2 As3 11_555 . 10_554 77.52(12) ?

02 Ca2 As3 11_555. 11_556 104.0(5) ?

02 Ca2 As3 11_555 . 4_556 90.29(14) ?

02 Ca2 As3 11_555 . 13_556 114.9(6) ?

02 Ca2 As3 11_555 . 6_546 81.40(13) ?

02 Ca2 As3 11_555 . 7_555 101.9(5) ?

02 Ca2 As3 11_555 . 16_544 67.58(9) ?

$02 \mathrm{Ca} 206$ 11_555 . 79.7(6) ?

02 Ca2 06 11_555 . 4_556 99.7(2) ?

02 Ca2 Ca1 11_555 . 9_445 90.4(3) ?

02 Ca2 Ca1 11_555 . 11 545 84.20(18) ?

02 Ca2 Mg2 11_555. . 0 ?

02 Ca2 Mg2 11_555 . 4_556 75(5) ?

02 Ca2 Mg2 11_555 . 6_546 127.4(3) ?

02 Ca2 Mg2 11_555 . 6_556 46.2(3) ?

02 Ca2 Mg2 11_555 . 7_545 130.6(3) ?

02 Ca2 Mg2 11_555 . 7_555 49.4(3) ?

02 Ca2 Ca2 11_555 . 4_556 75(5) ?

02 Ca2 Ca2 11_555 . 6_546 127.4(3) ?

02 Ca2 Ca2 11_555 . 6_556 46.2(3) ?

02 Ca2 Ca2 11_555 . 7_545 130.6(3) ? 


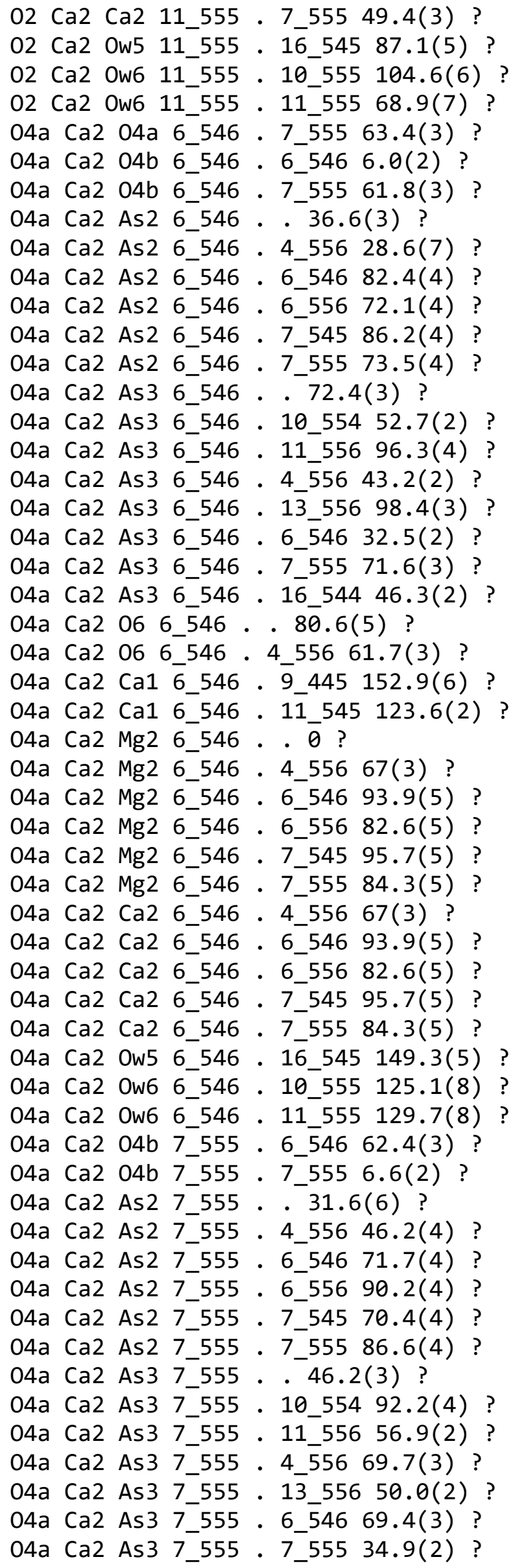




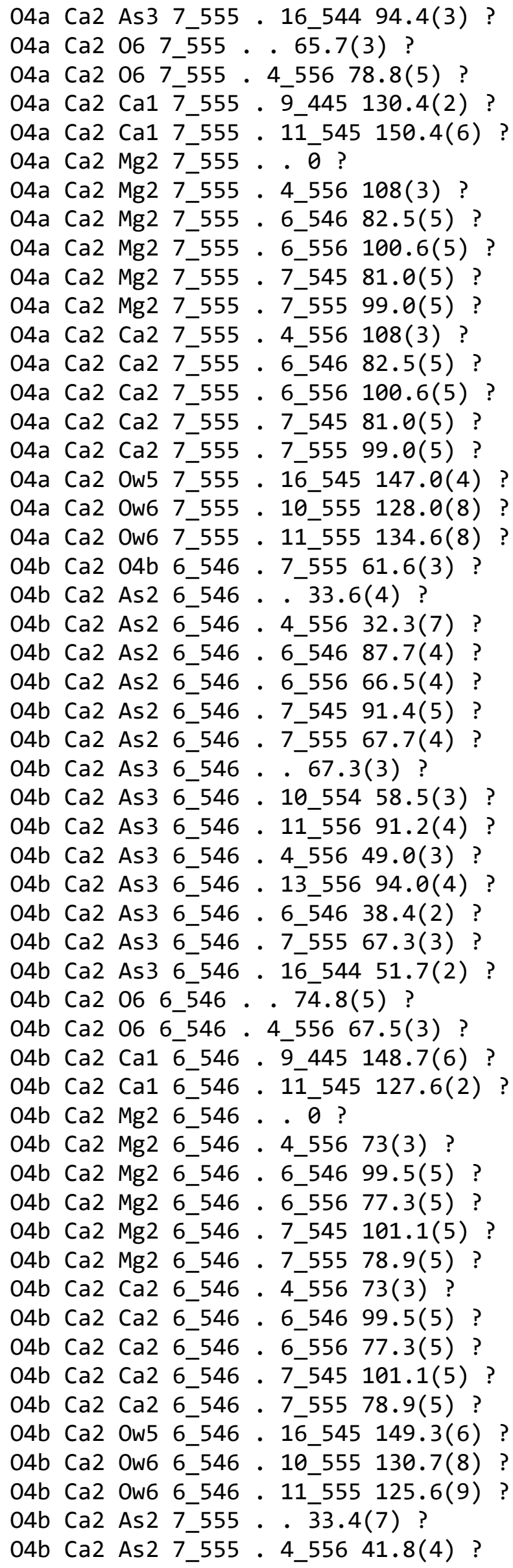




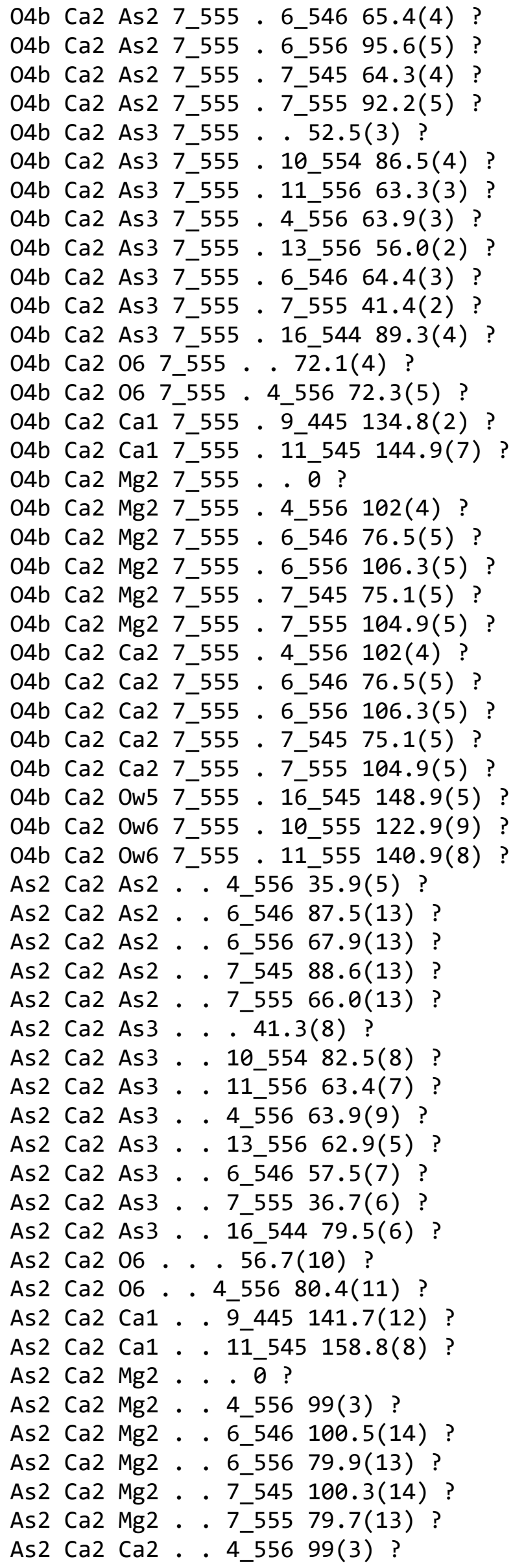




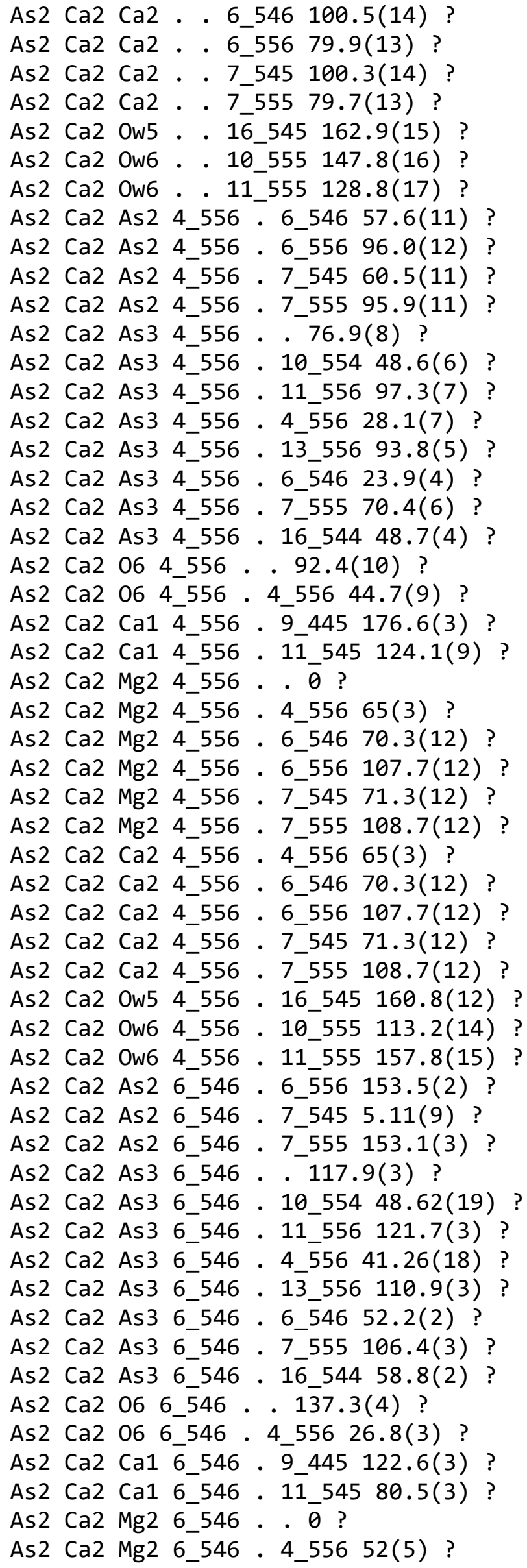




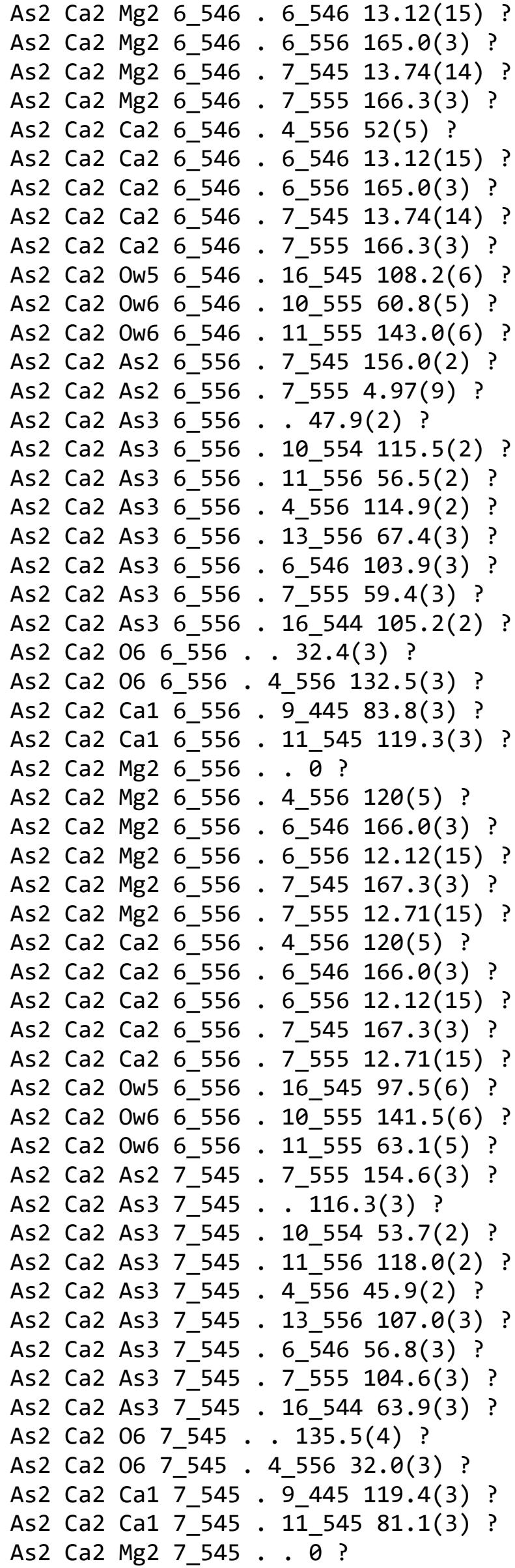




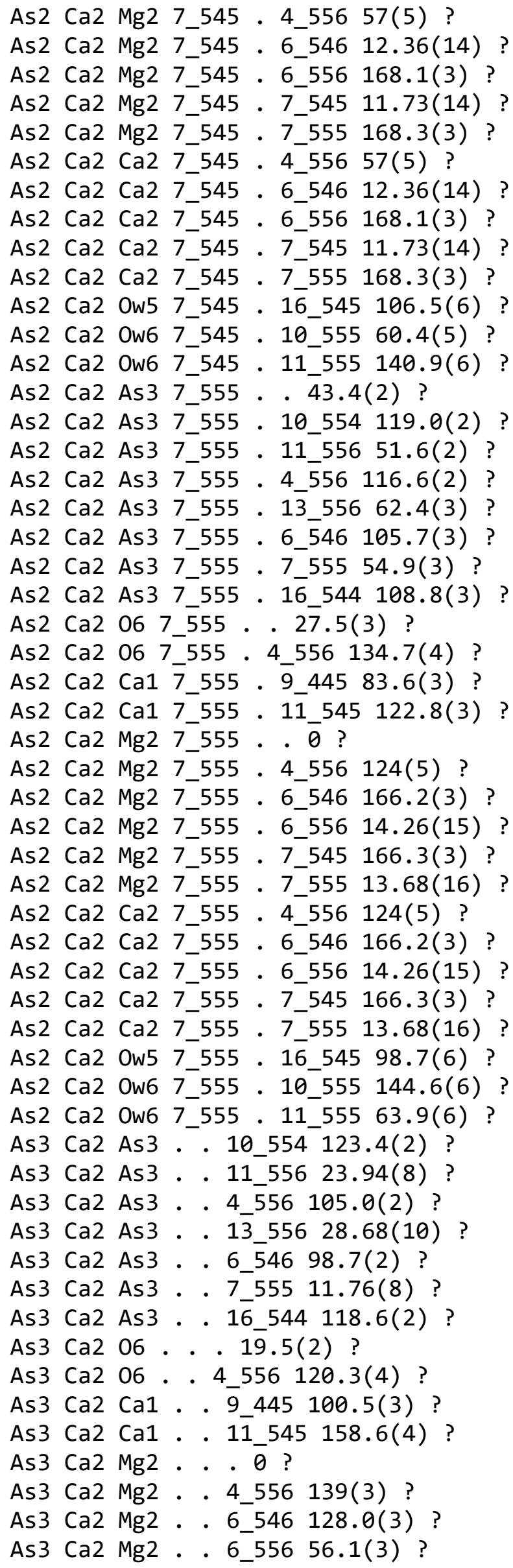




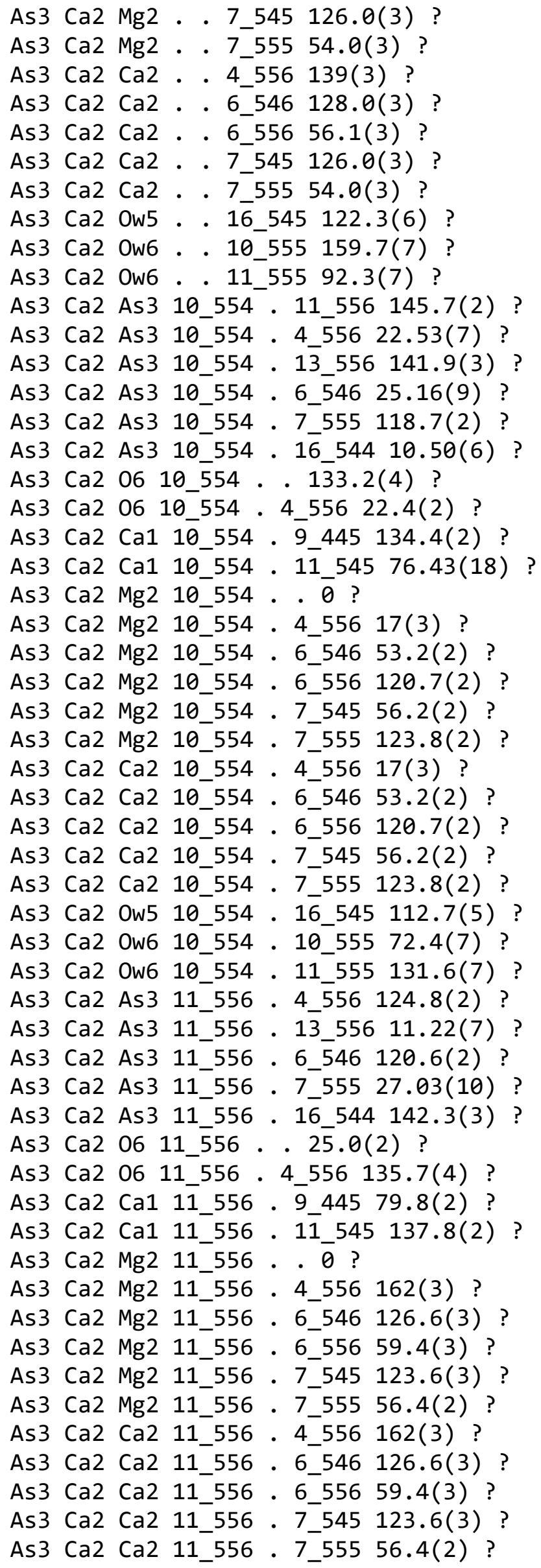




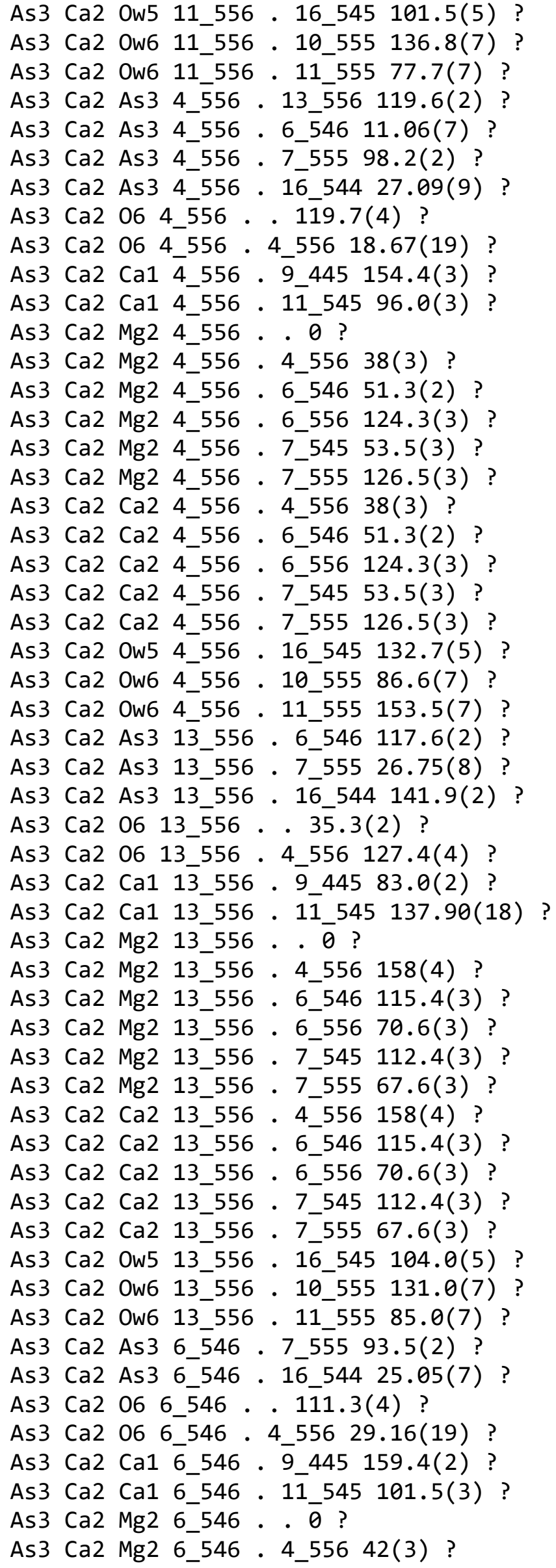




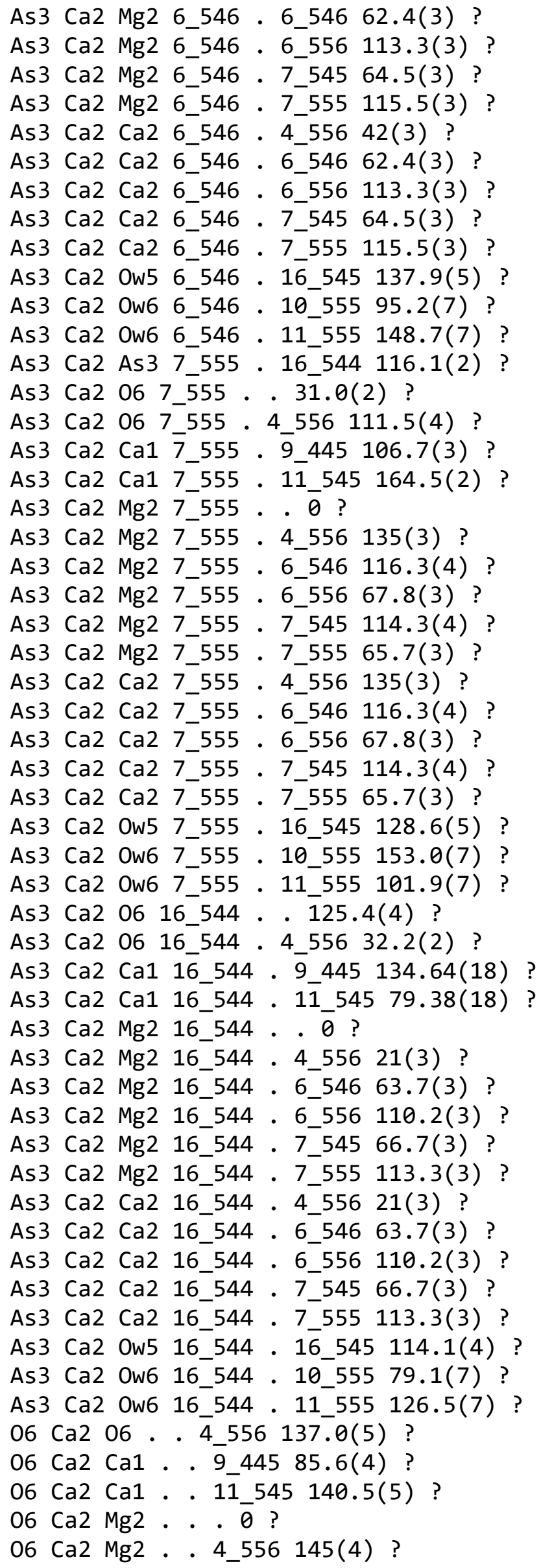




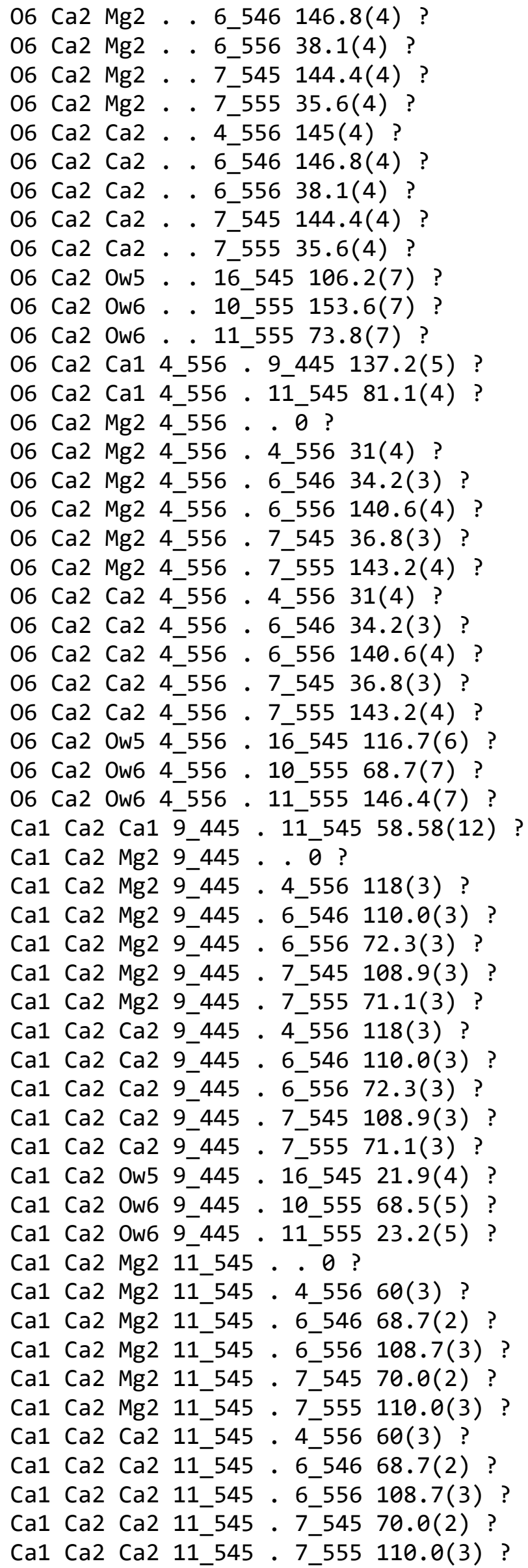


Ca1 Ca2 Ow5 11_545 - 16_545 36.7(4) ?

Ca1 Ca2 Ow6 11_545 . 10_555 22.5(5) ?

Ca1 Ca2 Ow6 11_545 . 11_555 66.7(5) ?

Mg2 Ca2 Mg2 . . 4_556 0 ?

Mg2 Ca2 Mg2 . . 6_546 0 ?

Mg2 Ca2 Mg2 . . 6_556 0 ?

Mg2 Ca2 Mg2 . . 7_545 0 ?

Mg2 Ca2 Mg2 . . 7_555 0 ?

Mg2 Ca2 Ca2 . 4 4_556 0 ?

Mg2 Ca2 Ca2 . . 6_546 0 ?

Mg2 Ca2 Ca2 . . 6_556 0 ?

Mg2 Ca2 Ca2 . 7 7545 0 ?

Mg2 Ca2 Ca2 . 75550 ?

Mg2 Ca2 Ow5 . . 16_545 0 ?

Mg2 Ca2 Ow6 . 10_555 0 ?

Mg2 Ca2 Ow6 . . 11_555 0 ?

Mg2 Ca2 Mg2 4_556 . 6_546 52(5) ?

Mg2 Ca2 Mg2 4_556 . 6_556 121(5) ?

Mg2 Ca2 Mg2 4_556 - 7_545 55(5) ?

Mg2 Ca2 Mg2 4_556 . 7_555 125(5) ?

Mg2 Ca2 Ca2 4_556 . 4_556 0.0(5) ?

Mg2 Ca2 Ca2 4_556 . 6_546 52(5) ?

Mg2 Ca2 Ca2 4_556 . 6_556 121(5) ?

Mg2 Ca2 Ca2 4_556 . 7_545 55(5) ?

Mg2 Ca2 Ca2 4_556 . 7_555 125(5) ?

Mg2 Ca2 Ow5 4_556 . 16_545 96(3) ?

Mg2 Ca2 Ow6 4_556 . 10_555 58(4) ?

Mg2 Ca2 Ow6 4_556 . 11_555 117(4) ?

Mg2 Ca2 Mg2 6_546 . 6_556 173.56(2) ?

Mg2 Ca2 Mg2 6_546 . 7_545 3.22(2) ?

Mg2 Ca2 Mg2 6_546 . 7_555 176.78(2) ?

Mg2 Ca2 Ca2 6_546 . 4_556 52(5) ?

Mg2 Ca2 Ca2 6_546 . 6_546 0.0(5) ?

Mg2 Ca2 Ca2 6_546 . 6_556 173.56(2) ?

Mg2 Ca2 Ca2 6_546 . 7_545 3.22(2) ?

Mg2 Ca2 Ca2 6_546 . 7_555 176.78(2) ?

Mg2 Ca2 Ow5 6_546 . 16_545 95.1(5) ?

Mg2 Ca2 Ow6 6_546 . 10_555 48.1(5) ?

Mg2 Ca2 Ow6 6_546 . 11_555 129.9(6) ?

Mg2 Ca2 Mg2 6_556 . 7_545 176.78(2) ?

Mg2 Ca2 Mg2 6_556 . 7_555 3.22(2) ?

Mg2 Ca2 Ca2 6_556 . 4_556 121(5) ?

Mg2 Ca2 Ca2 6_556 . 6_546 173.56(2) ?

Mg2 Ca2 Ca2 6_556 . 6_556 0.0(5) ?

Mg2 Ca2 Ca2 6_556 . 7_545 176.78(2) ?

Mg2 Ca2 Ca2 6_556 . 7_555 3.22(2) ?

Mg2 Ca2 Ow5 6_556 . 16_545 85.3(5) ?

Mg2 Ca2 Ow6 6_556 . 10_555 130.3(6) ?

Mg2 Ca2 Ow6 6_556 . 11_555 51.1(5) ?

Mg2 Ca2 Mg2 7_545 . 7_555 180.0(5) ?

Mg2 Ca2 Ca2 7_545 . 4_556 55(5) ?

Mg2 Ca2 Ca2 7_545 . 6_546 3.22(2) ?

Mg2 Ca2 Ca2 7_545 . 6_556 176.78(2) ?

Mg2 Ca2 Ca2 7_545 . 7_545 0.0(5) ? 


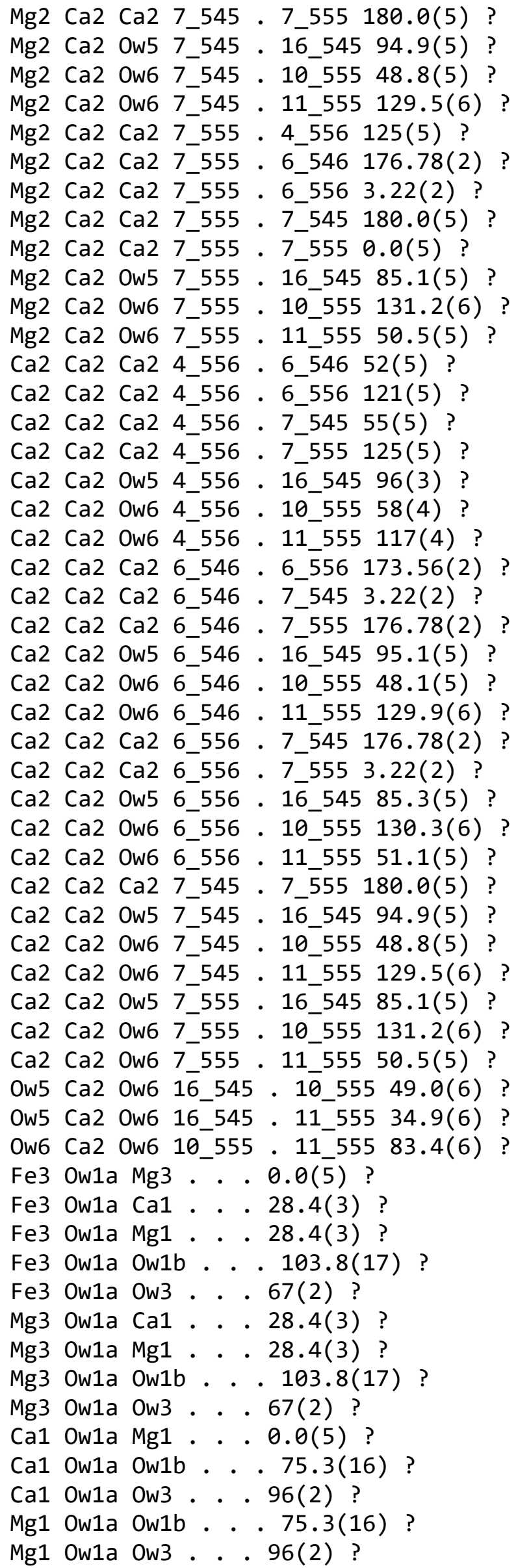




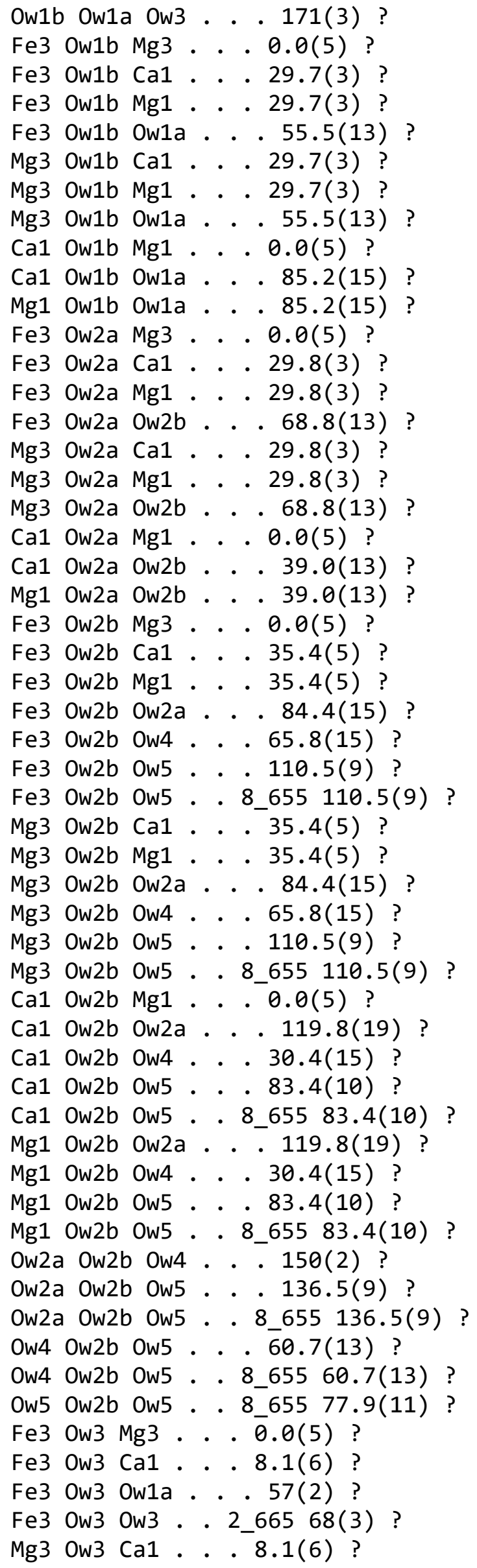




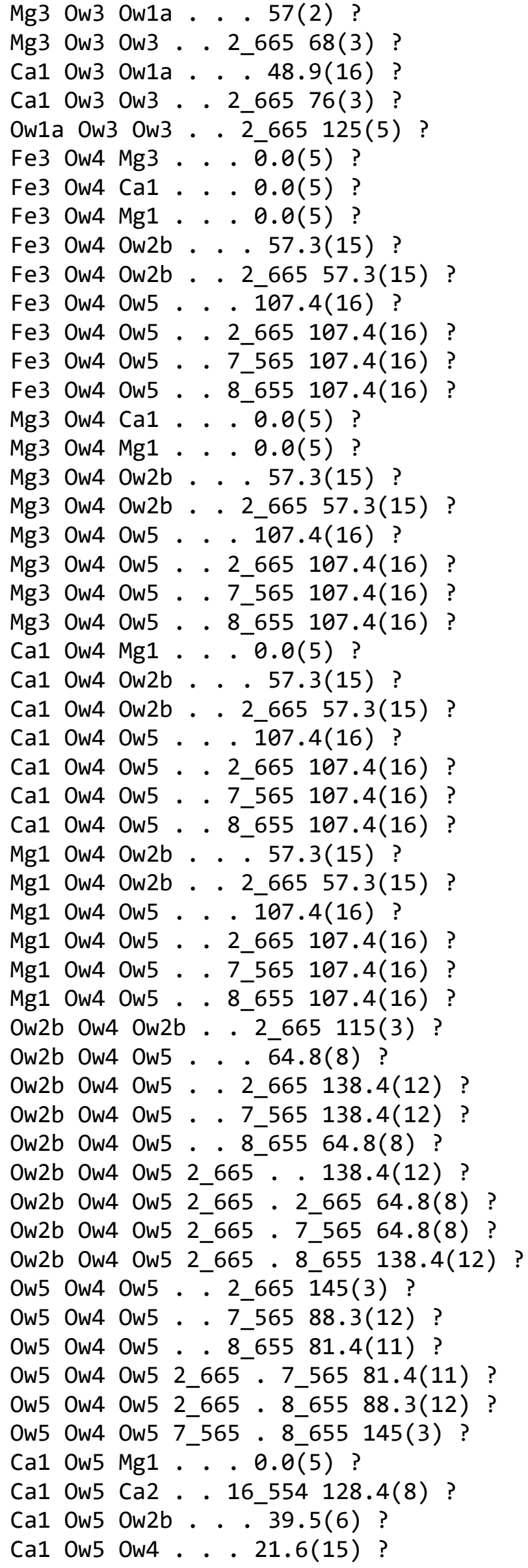




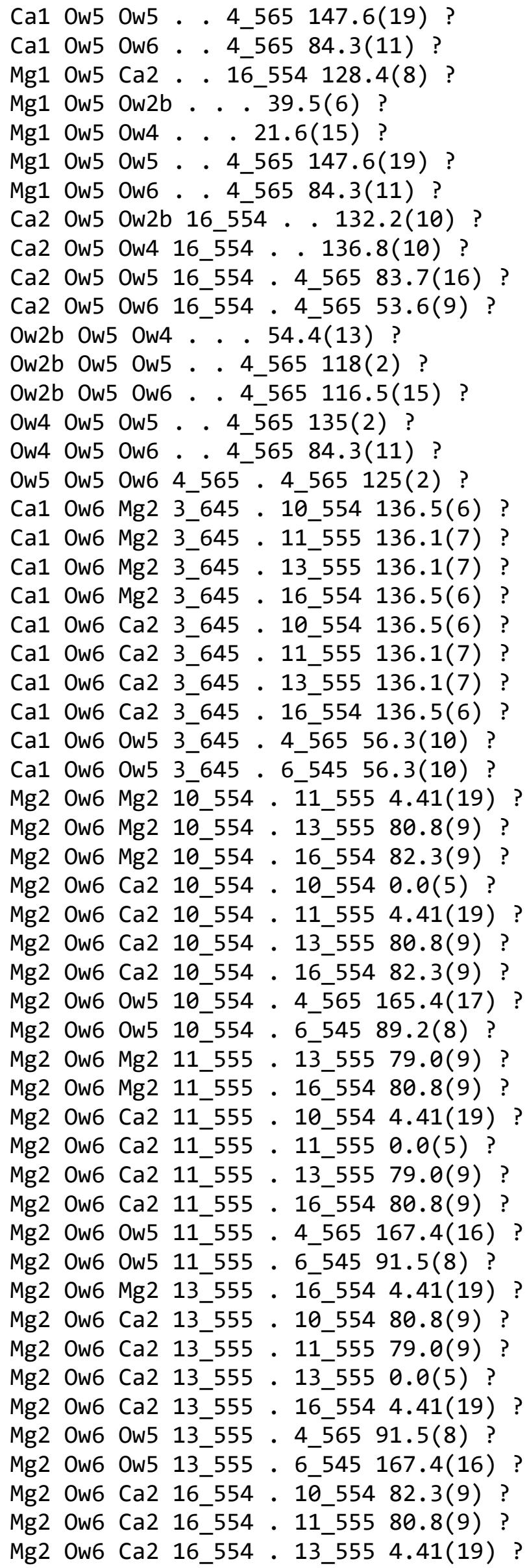


Mg2 Ow6 Ca2 16_554 . 16_554 0.0(5) ?

Mg2 Ow6 Ow5 16_554 . 4_565 89.2(8) ?

Mg2 Ow6 Ow5 16_554 . 6_545 165.4(17) ?

Ca2 Ow6 Ca2 10_554 . 11_555 4.41(19) ?

Ca2 Ow6 Ca2 10_554 . 13_555 80.8(9) ?

Ca2 Ow6 Ca2 10_554 . 16_554 82.3(9) ?

Ca2 Ow6 Ow5 10_554 . 4_565 165.4(17) ?

Ca2 Ow6 Ow5 10_554 . 6_545 89.2(8) ?

Ca2 Ow6 Ca2 11_555 . 13_555 79.0(9) ?

Ca2 Ow6 Ca2 11_555 . 16_554 80.8(9) ?

Ca2 Ow6 Ow5 11_555 . 4_565 167.4(16) ?

Ca2 Ow6 Ow5 11_555 . 6_545 91.5(8) ?

Ca2 Ow6 Ca2 13_555 . 16_554 4.41(19) ?

Ca2 Ow6 Ow5 13_555 . 4_565 91.5(8) ?

Ca2 Ow6 Ow5 13_555 . 6_545 167.4(16) ?

Ca2 Ow6 Ow5 16_554 . 4_565 89.2(8) ?

Ca2 Ow6 Ow5 16_554 . 6_545 165.4(17) ?

Ow5 Ow6 Ow5 4_565 . 6_545 96.5(16) ?

loop

_geom_torsion_atom_site_label_1

_geom_torsion_atom_site_label_2

_geom_torsion_atom_site_label_3

_geom_torsion_atom_site_label_4

_geom_torsion_site_symmetry_1

_geom_torsion_site_symmetry_2

_geom_torsion_site_symmetry_3

_geom_torsion_site_symmetry_4

_geom_torsion

_geom_torsion_publ_flag

? ? ? ? ? ? ? ? ? ?

loop_

_geom_hbond_atom_site_label_D

_geom_hbond_atom_site_label_H

_geom_hbond_atom_site_label_A

_geom_hbond_site_symmetry_D

_geom_hbond_site_symmetry_H

_geom_hbond_site_symmetry_A

_geom_hbond_distance_DH

_geom_hbond_distance_HA

_geom_hbond_distance_DA

_geom_hbond_angle_DHA

geom_hbond_publ_flag

? ? ? ? ? ? ? ? ? ? ?

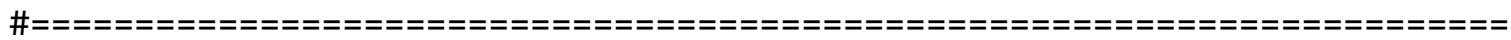

\# 11. STRUCTURE-FACTOR LIST

loop

_refiln_index_h

_refln_index_k 
_refln_index_l

_refln_F_squared_calc

_refln_F_squared_meas

_refln_F_squared_sigma

_refln_observed_status

\begin{tabular}{|c|c|c|c|c|c|}
\hline 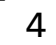 & 0 & 0 & 2371.29 & 2700.65 & 150.63 \\
\hline 6 & 0 & 0 & 8.95 & 166.77 & 102.22 \\
\hline 8 & 0 & 0 & 40235.00 & 45873.30 & 2049.69 \\
\hline 10 & 0 & 0 & 4909.01 & 5062.36 & 290.51 \\
\hline 12 & 0 & 0 & 1158.92 & 1231.97 & 166.77 \\
\hline & $\theta$ & $\theta$ & 1759.90 & 2759.82 & 225.95 \\
\hline & $\theta$ & 0 & 119670.00 & 108612.00 & 4831.03 \\
\hline & $\theta$ & $\theta$ & 4099.16 & 4782.62 & 225.95 \\
\hline . & $\theta$ & 0 & 80318.30 & 76059.20 & 3421.53 \\
\hline & $\theta$ & $\theta$ & 16220.30 & 14541.50 & 747.79 \\
\hline .4 & $\theta$ & 0 & 17696.10 & 18146.00 & 914.56 \\
\hline 26 & $\theta$ & 0 & 1238.10 & 1231.97 & 279.75 \\
\hline 28 & 0 & $\theta$ & 51.52 & -419.62 & 295.89 \\
\hline 30 & 0 & $\theta$ & 801.89 & 1194.31 & 193.67 \\
\hline 32 & 0 & $\theta$ & 9353.58 & 11195.30 & 693.99 \\
\hline 34 & 0 & $\theta$ & 18.42 & -161.39 & 376.58 \\
\hline$\theta$ & 2 & 0 & 20164.60 & 19786.80 & 634.81 \\
\hline 2 & 2 & $\theta$ & 12710.30 & 11760.20 & 317.41 \\
\hline 4 & 2 & $\theta$ & 16389.00 & 19291.90 & 392.72 \\
\hline 6 & 2 & $\theta$ & 55435.00 & 61453.10 & 1032.92 \\
\hline 8 & 2 & $\theta$ & 79276.60 & 67607.60 & 1140.51 \\
\hline 12 & 2 & $\theta$ & 129.49 & 204.43 & 48.42 \\
\hline .4 & 2 & $\theta$ & 761.26 & 667.09 & 53.80 \\
\hline 10 & 2 & $\theta$ & 24351.70 & 28313.70 & 575.64 \\
\hline 18 & 2 & $\theta$ & 5001.36 & 4072.49 & 145.25 \\
\hline 20 & 2 & $\theta$ & 4281.38 & 4018.69 & 134.49 \\
\hline 22 & 2 & 0 & 1521.65 & 1237.35 & 215.19 \\
\hline 24 & 2 & 0 & 9388.18 & 8279.47 & 478.80 \\
\hline 26 & 2 & $\theta$ & 726.14 & 780.07 & 247.47 \\
\hline 28 & 2 & $\theta$ & 5152.45 & 5869.33 & 317.41 \\
\hline 30 & 2 & $\theta$ & 374.13 & 500.32 & 225.95 \\
\hline 32 & 2 & $\theta$ & 4043.02 & 3937.99 & 312.03 \\
\hline 34 & 2 & $\theta$ & 1043.37 & 591.78 & 274.37 \\
\hline 0 & 4 & 0 & 439797.00 & 452482.00 & 14116.50 \\
\hline 2 & 4 & 0 & 46323.70 & 45012.50 & 1156.65 \\
\hline 4 & 4 & 0 & 1859.05 & 1490.20 & 96.84 \\
\hline 6 & 4 & 0 & 7808.42 & 8349.41 & 231.33 \\
\hline 8 & 4 & $\theta$ & 756.65 & 936.08 & 91.46 \\
\hline 10 & 4 & 0 & 3911.01 & 3755.08 & 129.12 \\
\hline 12 & 4 & 0 & 111.28 & 64.56 & 64.56 \\
\hline 14 & 4 & 0 & 3780.86 & 3114.89 & 107.60 \\
\hline 16 & 4 & $\theta$ & 51189.00 & 51247.70 & 1038.30 \\
\hline 18 & 4 & 0 & 9.95 & 53.80 & 91.46 \\
\hline 20 & 4 & 0 & 22408.20 & 20690.60 & 500.32 \\
\hline 22 & 4 & 0 & 2728.02 & 2878.18 & 139.87 \\
\hline 24 & 4 & 0 & 13472.30 & 14111.10 & 355.07 \\
\hline 26 & 4 & 0 & 3943.98 & 3529.13 & 193.67 \\
\hline 28 & 4 & $e$ & 1015.72 & 1312.66 & 166.77 \\
\hline 30 & 4 & $e$ & 1657.77 & 1775.32 & 263.61 \\
\hline
\end{tabular}




\begin{tabular}{|c|c|c|c|c|c|}
\hline 32 & 4 & 0 & 5907.24 & 8327.89 & 419.62 \\
\hline 0 & 6 & 0 & 16500.20 & 17425.10 & 511.08 \\
\hline 2 & 6 & $\theta$ & 3337.64 & 3819.64 & 156.01 \\
\hline 4 & 6 & $\theta$ & 10231.50 & 8344.03 & 242.09 \\
\hline 6 & 6 & 0 & 11543.70 & 10883.30 & 247.47 \\
\hline 8 & 6 & 0 & 58825.10 & 55831.30 & 989.88 \\
\hline 10 & 6 & 0 & 5226.86 & 4777.24 & 172.15 \\
\hline 12 & 6 & 0 & 4538.89 & 3674.38 & 161.39 \\
\hline 14 & 6 & 0 & 43.65 & 263.61 & 112.97 \\
\hline 16 & 6 & 0 & 24908.60 & 23934.60 & 457.28 \\
\hline 18 & 6 & 0 & 4919.68 & 4944.01 & 172.15 \\
\hline 20 & 6 & 0 & 359.66 & 521.84 & 123.74 \\
\hline 22 & 6 & 0 & 2911.30 & 2808.24 & 145.25 \\
\hline 24 & 6 & 0 & 13072.80 & 12846.90 & 451.90 \\
\hline 0 & 8 & 0 & 19769.00 & 18113.70 & 995.26 \\
\hline 2 & 8 & 0 & 503.48 & 290.51 & 338.93 \\
\hline 4 & 8 & 0 & 7063.65 & 5901.61 & 532.60 \\
\hline 6 & 8 & 0 & 6807.67 & 6068.38 & 365.82 \\
\hline 8 & 8 & 0 & 358.15 & 161.39 & 247.47 \\
\hline 10 & 8 & 0 & 218.42 & 494.94 & 258.23 \\
\hline 12 & 8 & 0 & 603.10 & 914.56 & 268.99 \\
\hline 14 & 8 & 0 & 5790.23 & 5820.91 & 371.20 \\
\hline 16 & 8 & 0 & 15105.20 & 15552.90 & 478.80 \\
\hline 18 & 8 & 0 & 1170.79 & 1178.17 & 285.13 \\
\hline 20 & 8 & 0 & 1057.11 & 1075.95 & 290.51 \\
\hline 1 & 0 & 1 & 18632.10 & 16860.20 & 435.76 \\
\hline 3 & 0 & 1 & 64760.30 & 51543.60 & 1312.66 \\
\hline 7 & 0 & 1 & 17786.50 & 19022.90 & 376.58 \\
\hline 9 & 0 & 1 & 4599.61 & 3652.86 & 96.84 \\
\hline 11 & 0 & 1 & 3719.98 & 3104.13 & 112.97 \\
\hline 13 & 0 & 1 & 5445.24 & 6052.24 & 193.67 \\
\hline 15 & 0 & 1 & 3815.88 & 3174.07 & 258. \\
\hline 17 & 0 & 1 & 46179.00 & 45270.80 & 1188.93 \\
\hline 19 & 0 & 1 & 44338.60 & 47971.40 & 1264.25 \\
\hline 21 & 0 & 1 & 4921.59 & 4664.26 & 199.05 \\
\hline 23 & 0 & 1 & 276.09 & 145.25 & 134.49 \\
\hline 25 & 0 & 1 & 1190.46 & 806.97 & 134.49 \\
\hline 27 & 0 & 1 & 941.34 & 1188.93 & 150.63 \\
\hline 29 & 0 & 1 & 275.86 & 102.22 & 150.63 \\
\hline 31 & 0 & 1 & 1124.09 & 1382.60 & 182.91 \\
\hline 33 & 0 & 1 & 244.89 & 462.66 & 258.23 \\
\hline 35 & 0 & 1 & 906.85 & 1425.64 & 295.89 \\
\hline 0 & 1 & 1 & 68055.90 & 60796.80 & 1904.44 \\
\hline 2 & 1 & 1 & 4870.91 & 6256.67 & 172.15 \\
\hline 4 & 1 & 1 & 81706.90 & 80314.60 & 1436.40 \\
\hline 6 & 1 & 1 & 2160.84 & 1979.76 & 43.04 \\
\hline 8 & 1 & 1 & 56960.50 & 51882.50 & 763.93 \\
\hline 10 & 1 & 1 & 14020.90 & 14676.00 & 204.43 \\
\hline 12 & 1 & 1 & 12826.50 & 11555.70 & 156.01 \\
\hline 14 & 1 & 1 & 13250.20 & 12341.20 & 161.39 \\
\hline 16 & 1 & 1 & 65044.90 & 61819.00 & 715.51 \\
\hline 18 & 1 & 1 & 15776.20 & 15633.60 & 312.03 \\
\hline 20 & 1 & 1 & 6892.64 & 6595.60 & 150.63 \\
\hline 22 & 1 & 1 & 6320.42 & 5761.74 & 139.87 \\
\hline
\end{tabular}




$\begin{array}{rrrrrrl}24 & 1 & 1 & 29297.00 & 30438.70 & 537.98 & \circ \\ 26 & 1 & 1 & 5754.83 & 5880.09 & 182.91 & \circ \\ 28 & 1 & 1 & 1271.19 & 1447.16 & 107.60 & \circ \\ 30 & 1 & 1 & 997.73 & 957.60 & 139.87 & \circ \\ 32 & 1 & 1 & 6645.14 & 8042.76 & 247.47 & \circ \\ 34 & 1 & 1 & 1138.58 & 1237.35 & 204.43 & \circ \\ 3 & 2 & 1 & 10472.50 & 9027.26 & 182.91 & \circ \\ 5 & 2 & 1 & 713.82 & 828.48 & 43.04 & \circ \\ 7 & 2 & 1 & 4553.25 & 4185.46 & 64.56 & \circ \\ 9 & 2 & 1 & 12013.60 & 10894.00 & 139.87 & \circ \\ 11 & 2 & 1 & 19928.10 & 19076.70 & 236.71 & \circ \\ 15 & 2 & 1 & 2665.00 & 2668.37 & 59.18 & \circ \\ 17 & 2 & 1 & 7843.17 & 8144.97 & 129.12 & \circ \\ 19 & 2 & 1 & 8271.24 & 8559.22 & 134.49 & \circ \\ 21 & 2 & 1 & 3332.14 & 2878.18 & 64.56 & \circ \\ 23 & 2 & 1 & 826.63 & 758.55 & 129.12 & \circ \\ 25 & 2 & 1 & 170.41 & 64.56 & 161.39 & < \\ 27 & 2 & 1 & 61.19 & 5.38 & 156.01 & < \\ 29 & 2 & 1 & 455.29 & 796.21 & 156.01 & \circ \\ 31 & 2 & 1 & 22.01 & 166.77 & 166.77 & < \\ 33 & 2 & 1 & 686.75 & 462.66 & 188.29 & < \\ 0 & 3 & 1 & 8357.44 & 7338.01 & 295.89 & \circ \\ 2 & 3 & 1 & 1105.03 & 1022.16 & 69.94 & \circ \\ 4 & 3 & 1 & 33395.30 & 34715.70 & 591.78 & \circ \\ 6 & 3 & 1 & 667.55 & 548.74 & 48.42 & \circ \\ 8 & 3 & 1 & 9193.58 & 8672.19 & 118.36 & \circ \\ 10 & 3 & 1 & 5745.80 & 5643.38 & 86.08 & \circ \\ 12 & 3 & 1 & 6738.04 & 6649.40 & 96.84 & \circ \\ 14 & 3 & 1 & 6248.12 & 6681.68 & 96.84 & \circ \\ 16 & 3 & 1 & 24830.20 & 22767.20 & 306.65 & \circ \\ 18 & 3 & 1 & 8776.04 & 8817.45 & 129.12 & \circ \\ 20 & 3 & 1 & 2923.50 & 2964.25 & 75.32 & \circ \\ 22 & 3 & 1 & 4552.56 & 4524.39 & 91.46 & \circ \\ 24 & 3 & 1 & 16199.00 & 15273.20 & 242.09 & \circ \\ 26 & 3 & 1 & 4794.90 & 4427.55 & 123.74 & \circ \\ 28 & 3 & 1 & 184.74 & 96.84 & 161.39 & < \\ 30 & 3 & 1 & 1491.26 & 1479.44 & 215.19 & \circ \\ 32 & 3 & 1 & 5119.27 & 5600.34 & 322.79 & \circ \\ 1 & 4 & 1 & 3164.00 & 3577.55 & 129.12 & \circ \\ 3 & 4 & 1 & 12490.70 & 12373.50 & 231.33 & \circ \\ 5 & 4 & 1 & 1479.51 & 1441.78 & 69.94 & \circ \\ 7 & 4 & 1 & 5565.86 & 6009.20 & 112.97 & \circ \\ 9 & 4 & 1 & 15023.60 & 15413.00 & 225.95 & \circ \\ 11 & 4 & 1 & 12149.00 & 13207.30 & 182.91 & \circ \\ 13 & 4 & 1 & 5133.82 & 5008.57 & 96.84 & \circ \\ 15 & 4 & 1 & 695.31 & 742.41 & 64.56 & \circ \\ 17 & 4 & 1 & 11215.60 & 11867.80 & 182.91 & \circ \\ 19 & 4 & 1 & 10439.20 & 10307.60 & 182.91 & \circ \\ 21 & 4 & 1 & 1138.20 & 984.50 & 86.08 & \circ \\ 23 & 4 & 1 & 38.19 & 21.52 & 80.70 & < \\ 27 & 4 & 1 & 11.83 & -64.56 & 80.70 & < \\ 29 & 4 & 1 & 86.12 & 118.36 & 107.60 & < \\ 31 & 4 & 1 & 132.83 & -64.56 & 252.85 & <\end{array}$




$\begin{array}{rrrrrrl}0 & 5 & 1 & 59953.20 & 55357.80 & 1038.30 & \circ \\ 2 & 5 & 1 & 10817.20 & 11168.40 & 204.43 & \circ \\ 4 & 5 & 1 & 9579.73 & 10695.00 & 236.71 & \circ \\ 6 & 5 & 1 & 7419.28 & 8032.00 & 139.87 & \circ \\ 8 & 5 & 1 & 45997.60 & 45394.50 & 597.16 & \circ \\ 10 & 5 & 1 & 8130.05 & 7897.50 & 129.12 & \circ \\ 12 & 5 & 1 & 6729.44 & 6778.51 & 118.36 & \circ \\ 14 & 5 & 1 & 2419.29 & 2700.65 & 75.32 & \circ \\ 16 & 5 & 1 & 23370.30 & 22444.40 & 328.17 & \circ \\ 18 & 5 & 1 & 2273.98 & 2125.01 & 86.08 & \circ \\ 20 & 5 & 1 & 9384.07 & 8688.33 & 193.67 & \circ \\ 22 & 5 & 1 & 151.65 & 16.14 & 86.08 & < \\ 24 & 5 & 1 & 8384.68 & 8666.81 & 215.19 & \circ \\ 26 & 5 & 1 & 476.33 & 624.05 & 139.87 & \circ \\ 28 & 5 & 1 & 3192.74 & 3529.13 & 295.89 & \circ \\ 30 & 5 & 1 & 2.79 & 451.90 & 252.85 & < \\ 1 & 6 & 1 & 0.00 & 10.76 & 80.70 & < \\ 3 & 6 & 1 & 831.06 & 850.00 & 107.60 & \circ \\ 5 & 6 & 1 & 3886.42 & 4029.45 & 112.97 & \circ \\ 7 & 6 & 1 & 127.50 & 91.46 & 91.46 & < \\ 9 & 6 & 1 & 8062.59 & 7757.63 & 172.15 & \circ \\ 11 & 6 & 1 & 8593.44 & 8349.41 & 166.77 & \circ \\ 13 & 6 & 1 & 0.35 & -53.80 & 86.08 & < \\ 15 & 6 & 1 & 1910.98 & 1958.24 & 86.08 & \circ \\ 17 & 6 & 1 & 518.36 & 468.04 & 86.08 & \circ \\ 19 & 6 & 1 & 112.94 & 199.05 & 86.08 & < \\ 21 & 6 & 1 & 292.25 & 231.33 & 112.97 & < \\ 23 & 6 & 1 & 8.41 & 247.47 & 139.87 & < \\ 25 & 6 & 1 & 816.95 & 930.70 & 161.39 & \circ \\ 0 & 7 & 1 & 23484.80 & 20168.80 & 591.78 & \circ \\ 2 & 7 & 1 & 7001.12 & 7278.83 & 236.71 & \circ \\ 4 & 7 & 1 & 1372.46 & 1667.73 & 145.25 & \circ \\ 6 & 7 & 1 & 4465.08 & 4481.35 & 161.39 & \circ \\ 8 & 7 & 1 & 13785.80 & 12647.80 & 295.89 & \circ \\ 10 & 7 & 1 & 2656.04 & 2706.02 & 123.74 & \circ \\ 12 & 7 & 1 & 3143.21 & 3545.27 & 150.63 & \circ \\ 14 & 7 & 1 & 617.74 & 828.48 & 96.84 & \circ \\ 16 & 7 & 1 & 6019.63 & 5056.99 & 139.87 & \circ \\ 18 & 7 & 1 & 461.27 & 564.88 & 134.49 & \circ \\ 20 & 7 & 1 & 3874.17 & 4110.15 & 209.81 & \circ \\ 22 & 7 & 1 & 99.32 & 220.57 & 247.47 & < \\ 24 & 7 & 1 & 3042.09 & 2619.95 & 290.51 & \circ \\ 1 & 8 & 1 & 544.26 & 570.26 & 129.12 & \circ \\ 3 & 8 & 1 & 2420.24 & 2168.05 & 166.77 & \circ \\ 5 & 8 & 1 & 1003.63 & 968.36 & 134.49 & \circ \\ 7 & 8 & 1 & 536.57 & 618.67 & 134.49 & \circ \\ 9 & 8 & 1 & 5440.88 & 5250.66 & 215.19 & \circ \\ 11 & 8 & 1 & 5562.15 & 5401.29 & 215.19 & \circ \\ 13 & 8 & 1 & 1822.25 & 2012.03 & 166.77 & \circ \\ 15 & 8 & 1 & 22.90 & 75.32 & 188.29 & < \\ 17 & 8 & 1 & 704.28 & 726.27 & 199.05 & \circ \\ 0 & 8 & 1 & 203.76 & 43.04 & 279.75 & < \\ 2 & 9 & 1 & 10456.80 & 8537.70 & 468.04 & \circ \\ & & 1 & 3268.44 & 3265.52 & 344.30 & \circ\end{array}$




$\begin{array}{rrrrrrr}4 & 9 & 1 & 35.18 & -86.08 & 215.19 & < \\ 6 & 9 & 1 & 2676.35 & 2227.23 & 236.71 & \circ \\ 8 & 9 & 1 & 7811.52 & 8274.09 & 333.55 & \circ \\ 10 & 9 & 1 & 1755.54 & 1942.10 & 193.67 & \circ \\ 12 & 9 & 1 & 594.92 & 1016.78 & 188.29 & \circ \\ 14 & 9 & 1 & 162.69 & 559.50 & 220.57 & < \\ 2 & 0 & 2 & 40741.20 & 27614.40 & 1237.35 & \circ \\ 4 & 0 & 2 & 2874.20 & 3211.72 & 107.60 & \circ \\ 6 & 0 & 2 & 70656.70 & 81907.00 & 1619.31 & \circ \\ 8 & 0 & 2 & 288110.00 & 287549.00 & 5153.82 & \circ \\ 10 & 0 & 2 & 26229.60 & 23902.30 & 478.80 & \circ \\ 12 & 0 & 2 & 58.52 & 145.25 & 48.42 & \circ \\ 14 & 0 & 2 & 8756.92 & 8284.85 & 182.91 & \circ \\ 16 & 0 & 2 & 36479.80 & 35194.50 & 683.23 & \circ \\ 18 & 0 & 2 & 3067.76 & 3211.72 & 91.46 & \circ \\ 20 & 0 & 2 & 2221.70 & 2383.24 & 123.74 & \circ \\ 22 & 0 & 2 & 860.42 & 408.86 & 107.60 & \circ \\ 24 & 0 & 2 & 13187.00 & 14030.40 & 328.17 & \circ \\ 26 & 0 & 2 & 3084.55 & 3324.70 & 161.39 & \circ \\ 28 & 0 & 2 & 12755.30 & 13406.40 & 419.62 & \circ \\ 30 & 0 & 2 & 2745.20 & 3018.05 & 236.71 & \circ \\ 32 & 0 & 2 & 3562.23 & 4610.46 & 468.04 & \circ \\ 34 & 0 & 2 & 833.17 & 919.94 & 408.86 & < \\ 1 & 1 & 2 & 77.31 & 451.90 & 59.18 & \circ \\ 3 & 1 & 2 & 503.18 & 753.17 & 64.56 & \circ \\ 5 & 1 & 2 & 8975.69 & 10544.40 & 247.47 & \circ \\ 7 & 1 & 2 & 21148.90 & 21960.20 & 376.58 & \circ \\ 9 & 1 & 2 & 20388.70 & 22299.20 & 355.07 & \circ \\ 11 & 1 & 2 & 12913.40 & 13476.30 & 182.91 & \circ \\ 13 & 1 & 2 & 7551.11 & 7526.30 & 107.60 & \circ \\ 15 & 1 & 2 & 4928.34 & 5035.47 & 75.32 & \circ \\ 17 & 1 & 2 & 4.05 & -16.14 & 37.66 & < \\ 19 & 1 & 2 & 197.19 & 290.51 & 43.04 & \circ \\ 21 & 1 & 2 & 980.48 & 1097.47 & 75.32 & \circ \\ 23 & 1 & 2 & 919.89 & 801.59 & 75.32 & \circ \\ 25 & 1 & 2 & 2181.16 & 2431.66 & 118.36 & \circ \\ 27 & 1 & 2 & 3421.03 & 3324.70 & 134.49 & \circ \\ 29 & 1 & 2 & 196.45 & 317.41 & 166.77 & < \\ 31 & 1 & 2 & 322.94 & 193.67 & 182.91 & < \\ 33 & 1 & 2 & 961.48 & 1361.08 & 285.13 & \circ \\ 0 & 2 & 2 & 502452.00 & 532006.00 & 16564.30 & \circ \\ 2 & 2 & 2 & 111270.00 & 114379.00 & 3577.55 & \circ \\ 4 & 2 & 2 & 3590.43 & 3464.57 & 112.97 & \circ \\ 6 & 2 & 2 & 400.27 & 220.57 & 48.42 & \circ \\ 10 & 2 & 2 & 8.20 & 5.38 & 37.66 & < \\ 12 & 2 & 2 & 3237.12 & 2937.36 & 59.18 & \circ \\ 14 & 2 & 2 & 4039.20 & 3948.75 & 69.94 & \circ \\ 16 & 2 & 2 & 42770.70 & 35641.00 & 468.04 & \circ \\ 18 & 2 & 2 & 20.54 & 107.60 & 43.04 & < \\ 20 & 2 & 2 & 30761.00 & 29836.20 & 338.93 & \circ \\ 22 & 2 & 2 & 1587.67 & 1301.90 & 48.42 & \circ \\ 24 & 2 & 2 & 13542.50 & 13530.10 & 263.61 & \circ \\ 26 & 2 & 2 & 3777.41 & 3733.56 & 204.43 & \circ \\ 28 & 2 & 2 & 45.35 & 32.28 & 274.37 & < \\ & & & & & & \end{array}$




$\begin{array}{rrrrrrl}30 & 2 & 2 & 1759.44 & 1893.68 & 225.95 & \circ \\ 32 & 2 & 2 & 6371.88 & 6826.93 & 301.27 & \circ \\ 34 & 2 & 2 & 443.58 & 720.89 & 279.75 & < \\ 1 & 3 & 2 & 6803.42 & 8010.48 & 177.53 & \circ \\ 3 & 3 & 2 & 3488.07 & 4777.24 & 123.74 & \circ \\ 5 & 3 & 2 & 2971.98 & 3061.09 & 86.08 & \circ \\ 7 & 3 & 2 & 7013.39 & 6466.48 & 107.60 & \circ \\ 9 & 3 & 2 & 9.84 & -5.38 & 48.42 & < \\ 11 & 3 & 2 & 108.35 & 118.36 & 48.42 & < \\ 13 & 3 & 2 & 5714.40 & 6030.72 & 102.22 & \circ \\ 15 & 3 & 2 & 2682.89 & 2350.96 & 69.94 & \circ \\ 17 & 3 & 2 & 4586.29 & 4427.55 & 91.46 & \circ \\ 19 & 3 & 2 & 3409.95 & 2948.11 & 80.70 & \circ \\ 21 & 3 & 2 & 821.21 & 844.62 & 59.18 & \circ \\ 23 & 3 & 2 & 381.35 & 392.72 & 53.80 & \circ \\ 25 & 3 & 2 & 27.73 & 75.32 & 59.18 & < \\ 27 & 3 & 2 & 294.13 & 430.38 & 91.46 & \circ \\ 29 & 3 & 2 & 250.44 & 107.60 & 225.95 & < \\ 31 & 3 & 2 & 264.42 & 349.68 & 312.03 & < \\ 33 & 3 & 2 & 51.38 & 225.95 & 236.71 & < \\ 0 & 4 & 2 & 3758.37 & 4185.46 & 139.87 & \circ \\ 2 & 4 & 2 & 16601.00 & 15402.30 & 371.20 & \circ \\ 4 & 4 & 2 & 4556.05 & 4965.53 & 123.74 & \circ \\ 6 & 4 & 2 & 30229.30 & 32515.30 & 446.52 & \circ \\ 8 & 4 & 2 & 121929.00 & 123713.00 & 1640.83 & \circ \\ 10 & 4 & 2 & 14115.90 & 13072.80 & 177.53 & \circ \\ 12 & 4 & 2 & 1573.20 & 1538.61 & 64.56 & \circ \\ 14 & 4 & 2 & 2276.63 & 2307.92 & 69.94 & \circ \\ 16 & 4 & 2 & 22786.20 & 22455.20 & 322.79 & \circ \\ 18 & 4 & 2 & 4377.92 & 4158.56 & 107.60 & \circ \\ 20 & 4 & 2 & 61.14 & 59.18 & 75.32 & < \\ 22 & 4 & 2 & 2926.28 & 2598.43 & 91.46 & \circ \\ 24 & 4 & 2 & 12458.70 & 12927.60 & 225.95 & \circ \\ 26 & 4 & 2 & 376.84 & 489.56 & 96.84 & \circ \\ 28 & 4 & 2 & 5879.20 & 5745.60 & 242.09 & \circ \\ 30 & 4 & 2 & 624.11 & 774.69 & 258.23 & < \\ 32 & 4 & 2 & 3380.19 & 3152.55 & 381.96 & \circ \\ 1 & 5 & 2 & 2438.71 & 1888.30 & 91.46 & \circ \\ 3 & 5 & 2 & 3199.35 & 3674.38 & 129.12 & \circ \\ 5 & 5 & 2 & 731.84 & 758.55 & 86.08 & \circ \\ 7 & 5 & 2 & 1431.11 & 1387.98 & 69.94 & \circ \\ 9 & 5 & 2 & 18746.70 & 18205.10 & 247.47 & \circ \\ 11 & 5 & 2 & 16265.30 & 15967.20 & 215.19 & \circ \\ 13 & 5 & 2 & 30.91 & -10.76 & 59.18 & < \\ 15 & 5 & 2 & 342.40 & 446.52 & 75.32 & \circ \\ 17 & 5 & 2 & 1946.04 & 1651.59 & 86.08 & \circ \\ 19 & 5 & 2 & 667.82 & 489.56 & 86.08 & \circ \\ 21 & 5 & 2 & 330.96 & 242.09 & 80.70 & < \\ 23 & 5 & 2 & 752.05 & 936.08 & 86.08 & \circ \\ 25 & 5 & 2 & 1194.16 & 1366.46 & 118.36 & \circ \\ 0 & 5 & 2 & 1552.79 & 1662.35 & 188.29 & \circ \\ 2 & 6 & 2 & 406.31 & 365.82 & 247.47 & < \\ & 6 & 2 & 8371.23 & 7908.26 & 182.91 & \circ\end{array}$




$\begin{array}{rrrrrrl}4 & 6 & 2 & 7627.98 & 7020.60 & 188.29 & \circ \\ 6 & 6 & 2 & 2735.87 & 2921.22 & 107.60 & \circ \\ 8 & 6 & 2 & 1576.60 & 1635.45 & 86.08 & \circ \\ 10 & 6 & 2 & 7.34 & 156.01 & 75.32 & < \\ 12 & 6 & 2 & 198.59 & 231.33 & 75.32 & \circ \\ 14 & 6 & 2 & 4454.98 & 4524.39 & 112.97 & \circ \\ 16 & 6 & 2 & 24498.20 & 25580.80 & 414.24 & \circ \\ 18 & 6 & 2 & 1042.20 & 1221.21 & 107.60 & \circ \\ 20 & 6 & 2 & 5178.28 & 5320.59 & 161.39 & \circ \\ 22 & 6 & 2 & 6.22 & 91.46 & 118.36 & < \\ 24 & 6 & 2 & 10287.50 & 9974.10 & 247.47 & \circ \\ 26 & 6 & 2 & 3614.36 & 3889.57 & 225.95 & \circ \\ 1 & 7 & 2 & 17.52 & -37.66 & 112.97 & < \\ 3 & 7 & 2 & 184.77 & 59.18 & 107.60 & < \\ 5 & 7 & 2 & 460.70 & 96.84 & 102.22 & < \\ 7 & 7 & 2 & 301.74 & 236.71 & 96.84 & < \\ 9 & 7 & 2 & 1757.89 & 1963.62 & 118.36 & \circ \\ 11 & 7 & 2 & 1760.22 & 1678.49 & 118.36 & \circ \\ 13 & 7 & 2 & 107.97 & -134.49 & 161.39 & < \\ 15 & 7 & 2 & 15.02 & -91.46 & 161.39 & < \\ 17 & 7 & 2 & 164.54 & 376.58 & 156.01 & < \\ 19 & 7 & 2 & 83.91 & 242.09 & 161.39 & < \\ 21 & 7 & 2 & 52.04 & 139.87 & 247.47 & < \\ 23 & 7 & 2 & 19.75 & 188.29 & 252.85 & < \\ 0 & 8 & 2 & 100.60 & 215.19 & 188.29 & < \\ 2 & 8 & 2 & 2871.82 & 2991.15 & 182.91 & \circ \\ 4 & 8 & 2 & 2244.13 & 2286.40 & 177.53 & \circ \\ 6 & 8 & 2 & 4817.71 & 4987.05 & 166.77 & \circ \\ 8 & 8 & 2 & 19926.30 & 20701.40 & 408.86 & \circ \\ 10 & 8 & 2 & 1583.56 & 1490.20 & 139.87 & \circ \\ 12 & 8 & 2 & 2929.26 & 3157.93 & 161.39 & \circ \\ 14 & 8 & 2 & 576.82 & 898.42 & 193.67 & \circ \\ 16 & 8 & 2 & 5478.12 & 5288.32 & 252.85 & \circ \\ 18 & 8 & 2 & 2950.39 & 2867.42 & 312.03 & \circ \\ 20 & 8 & 2 & 647.67 & 946.84 & 295.89 & \circ \\ 1 & 9 & 2 & 40.45 & -86.08 & 242.09 & < \\ 3 & 9 & 2 & 121.85 & 564.88 & 225.95 & < \\ 5 & 9 & 2 & 57.82 & 86.08 & 161.39 & < \\ 7 & 9 & 2 & 124.22 & 48.42 & 182.91 & < \\ 9 & 9 & 2 & 1964.39 & 2211.09 & 204.43 & \circ \\ 11 & 9 & 2 & 1927.61 & 1866.78 & 236.71 & \circ \\ 13 & 9 & 2 & 331.98 & 172.15 & 317.41 & < \\ 1 & 0 & 3 & 7205.66 & 6509.52 & 199.05 & \circ \\ 7 & 0 & 3 & 2554.68 & 2888.94 & 80.70 & \circ \\ 9 & 0 & 3 & 163678.00 & 171911.00 & 3098.75 & \circ \\ 11 & 0 & 3 & 174521.00 & 186737.00 & 3356.98 & \circ \\ 13 & 0 & 3 & 6612.47 & 7919.02 & 204.43 & \circ \\ 15 & 0 & 3 & 2430.80 & 2377.86 & 96.84 & \circ \\ 17 & 0 & 3 & 545.52 & 398.10 & 69.94 & \circ \\ 19 & 0 & 3 & 139.92 & 537.98 & 75.32 & \circ \\ 21 & 0 & 3 & 4275.09 & 3798.12 & 96.84 & \circ \\ 23 & 0 & 3 & 2198.39 & 2044.31 & 123.74 & \circ \\ 25 & 0 & 3 & 2116.36 & 2420.90 & 172.15 & \circ \\ 27 & 0 & 3 & 2228.13 & 2173.43 & 177.530 & \circ\end{array}$




\begin{tabular}{|c|c|c|c|c|c|}
\hline 29 & $\theta$ & 3 & 3436.92 & 3636.73 & 215.19 \\
\hline 31 & 0 & 3 & 3423.92 & 3787.36 & 451.90 \\
\hline 33 & 0 & 3 & 778.89 & 774.69 & 403.48 \\
\hline 0 & 1 & 3 & 70964.10 & 91875.70 & 2878.18 \\
\hline 4 & 1 & 3 & 19440.70 & 21922.60 & 451.90 \\
\hline 6 & 1 & & 121.91 & 193.67 & 37.66 \\
\hline 8 & 1 & & 11403.00 & 9549.09 & 166.77 \\
\hline 10 & 1 & & 1783.98 & 1882.92 & 53.80 \\
\hline 12 & 1 & & 5652.62 & 5869.33 & 107.60 \\
\hline 14 & 1 & & 6330.00 & 6853.83 & 123.74 \\
\hline 16 & 1 & 3 & 35677.20 & 36953.70 & 435.76 \\
\hline 18 & 1 & 3 & 5443.74 & 4685.78 & 75.32 \\
\hline 20 & 1 & 3 & 2951.13 & 2657.61 & 64.56 \\
\hline 22 & 1 & 3 & 2081.06 & 2173.43 & 59.18 \\
\hline 24 & 1 & 3 & 15340.60 & 14961.10 & 317.41 \\
\hline 26 & 1 & 3 & 5558.92 & 5810.15 & 215.19 \\
\hline 28 & 1 & 3 & 22.41 & 112.97 & 145.25 \\
\hline 30 & 1 & 3 & 1462.94 & 1447.16 & 193.67 \\
\hline 32 & 1 & 3 & 6269.26 & 7311.11 & 312.03 \\
\hline 34 & 1 & 3 & 1057.36 & 769.31 & 290.51 \\
\hline 1 & 2 & 3 & 2903.68 & 2636.09 & 156.01 \\
\hline 5 & 2 & 3 & 701.04 & 425.00 & 64.56 \\
\hline 7 & 2 & 3 & 11902.60 & 12884.60 & 220.57 \\
\hline 9 & 2 & 3 & 2474.43 & 2759.82 & 69.94 \\
\hline 11 & 2 & 3 & 2805.57 & 3093.37 & 69.94 \\
\hline 13 & 2 & 3 & 6067.32 & 6869.97 & 118.36 \\
\hline 15 & 2 & 3 & 195.36 & 236.71 & 43.04 \\
\hline 17 & 2 & 3 & 16008.00 & 17113.10 & 220.57 \\
\hline 19 & 2 & 3 & 11832.50 & 12647.80 & 161.39 \\
\hline 21 & 2 & 3 & 837.04 & 715.51 & 48.42 \\
\hline 23 & 2 & 3 & 44.35 & 26.90 & 48.42 \\
\hline 25 & 2 & 3 & 563.83 & 398.10 & 53.80 \\
\hline 27 & 2 & 3 & 792.13 & 1221.21 & 172.15 \\
\hline 29 & 2 & 3 & 1184.63 & 1495.58 & 188.29 \\
\hline 31 & 2 & 3 & 2503.00 & 3055.71 & 268.99 \\
\hline 33 & 2 & 3 & 274.24 & 672.47 & 215.19 \\
\hline$\theta$ & 3 & 3 & 104650.00 & 107445.00 & 2759.82 \\
\hline 2 & 3 & 3 & 21816.50 & 23638.70 & 634.81 \\
\hline 4 & 3 & 3 & 15086.80 & 15977.90 & 387.34 \\
\hline 6 & 3 & 3 & 4925.32 & 5815.53 & 129.12 \\
\hline 8 & 3 & 3 & 45216.90 & 43253.40 & 688.61 \\
\hline 10 & 3 & 3 & 8205.21 & 8860.48 & 177.53 \\
\hline 12 & 3 & 3 & 5874.21 & 5976.93 & 118.36 \\
\hline 14 & 3 & 3 & 8527.57 & 7601.62 & 134.49 \\
\hline 16 & 3 & 3 & 39555.70 & 41935.30 & 640.19 \\
\hline 18 & 3 & 3 & 4454.29 & 4115.52 & 96.84 \\
\hline 20 & 3 & 3 & 8077.09 & 7569.34 & 129.12 \\
\hline 22 & 3 & 3 & 626.78 & 650.95 & 64.56 \\
\hline 24 & 3 & 3 & 11300.80 & 11125.40 & 161.39 \\
\hline 26 & 3 & 3 & 1863.23 & 2092.73 & 80.70 \\
\hline 28 & 3 & 3 & 1509.12 & 1506.34 & 102.22 \\
\hline 30 & 3 & 3 & 192.13 & 37.66 & 199.05 \\
\hline 32 & 3 & 3 & 4159.66 & 5003.19 & 322.79 \\
\hline 1 & 4 & 3 & 34.94 & 107.60 & 64.56 \\
\hline
\end{tabular}




$\begin{array}{rrrrrrr}3 & 4 & 3 & 1651.05 & 2092.73 & 102.22 & 0 \\ 5 & 4 & 3 & 10965.30 & 11055.40 & 220.57 & 0 \\ 7 & 4 & 3 & 14.26 & 123.74 & 64.56 & < \\ 9 & 4 & 3 & 35954.20 & 35915.40 & 494.94 & 0 \\ 11 & 4 & 3 & 39019.70 & 40472.00 & 554.12 & 0 \\ 13 & 4 & 3 & 285.36 & 430.38 & 64.56 & 0 \\ 15 & 4 & 3 & 4667.27 & 4831.03 & 107.60 & 0 \\ 17 & 4 & 3 & 1447.69 & 1425.64 & 75.32 & 0 \\ 19 & 4 & 3 & 1560.11 & 1420.26 & 75.32 & 0 \\ 21 & 4 & 3 & 2534.22 & 2835.14 & 91.46 & 0 \\ 23 & 4 & 3 & 571.14 & 398.10 & 75.32 & 0 \\ 25 & 4 & 3 & 1153.88 & 1075.95 & 102.22 & 0 \\ 27 & 4 & 3 & 1098.59 & 1226.59 & 139.87 & 0 \\ 29 & 4 & 3 & 1215.53 & 1624.69 & 215.19 & 0 \\ 31 & 4 & 3 & 1234.20 & 973.74 & 220.57 & 0 \\ 0 & 5 & 3 & 33534.10 & 33193.20 & 693.99 & 0 \\ 2 & 5 & 3 & 5709.68 & 5336.73 & 145.25 & 0 \\ 4 & 5 & 3 & 2157.82 & 1807.60 & 102.22 & 0 \\ 6 & 5 & 3 & 187.25 & 182.91 & 69.94 & < \\ 8 & 5 & 3 & 5672.48 & 5562.68 & 118.36 & 0 \\ 10 & 5 & 3 & 382.56 & 403.48 & 69.94 & 0 \\ 12 & 5 & 3 & 3054.31 & 3012.67 & 91.46 & 0 \\ 14 & 5 & 3 & 1004.59 & 806.97 & 75.32 & 0 \\ 16 & 5 & 3 & 10657.90 & 10490.60 & 166.77 & 0 \\ 18 & 5 & 3 & 940.33 & 989.88 & 80.70 & 0 \\ 20 & 5 & 3 & 1938.68 & 2038.93 & 96.84 & 0 \\ 22 & 5 & 3 & 319.83 & 403.48 & 91.46 & 0 \\ 24 & 5 & 3 & 5766.48 & 6036.10 & 156.01 & 0 \\ 26 & 5 & 3 & 2238.79 & 2512.35 & 177.53 & 0 \\ 28 & 5 & 3 & 38.66 & 59.18 & 150.63 & < \\ 1 & 6 & 3 & 5503.44 & 5433.57 & 139.87 & 0 \\ 3 & 6 & 3 & 8886.46 & 8591.50 & 215.19 & 0 \\ 5 & 6 & 3 & 1263.97 & 1576.27 & 96.84 & 0 \\ 7 & 6 & 3 & 9116.05 & 9430.74 & 177.53 & 0 \\ 9 & 6 & 3 & 977.77 & 1221.21 & 86.08 & 0 \\ 10 & 7 & 3 & 2777.92 & 3335.46 & 129.12 & 0 \\ 12 & 7 & 3 & 850.80 & 968.36 & 112.97 & 0 \\ 14 & 7 & 3 & 1496.26 & 1178.17 & 166.77 & 0 \\ 16 & 7 & 3 & 7770.29 & 7913.64 & 274.37 & 0 \\ 18 & 7 & 3 & 585.85 & 559.50 & 166.77 & 0 \\ 13 & 6 & 3 & 940.32 & 1231.97 & 96.84 & 0 \\ 15 & 6 & 3 & 7426.94 & 7085.16 & 150.63 & 0 \\ 17 & 6 & 3 & 963.73 & 973.74 & 96.84 & 0 \\ 19 & 6 & 3 & 5996.04 & 5987.69 & 139.87 & 0 \\ 21 & 6 & 3 & 4265.05 & 4206.98 & 134.49 & 0 \\ 23 & 6 & 3 & 163.31 & 172.15 & 107.60 & < \\ 25 & 6 & 3 & 546.90 & 866.14 & 118.36 & 0 \\ 27 & 6 & 3 & 1708.79 & 2022.79 & 215.19 & 0 \\ 0 & 7 & 3 & 20443.10 & 19146.60 & 586.40 & 0 \\ 2 & 7 & 3 & 4820.66 & 5191.48 & 177.53 & 0 \\ 4 & 7 & 3 & 1354.55 & 1570.89 & 139.87 & 0 \\ 6 & 7 & 3 & 2009.56 & 2076.59 & 139.87 & 0 \\ 8 & 7 & 3 & 11985.60 & 11179.20 & 258.23 & 0\end{array}$




$\begin{array}{rrrrrrr}20 & 7 & 3 & 3098.81 & 3695.90 & 209.81 & \circ \\ 22 & 7 & 3 & 12.85 & -59.18 & 156.01 & < \\ 24 & 7 & 3 & 1914.51 & 2264.88 & 279.75 & \circ \\ 1 & 8 & 3 & 352.39 & 441.14 & 161.39 & < \\ 3 & 8 & 3 & 3187.67 & 3044.95 & 150.63 & \circ \\ 5 & 8 & 3 & 7339.96 & 7338.01 & 204.43 & \circ \\ 7 & 8 & 3 & 2206.08 & 2146.53 & 134.49 & \circ \\ 9 & 8 & 3 & 638.26 & 521.84 & 139.87 & \circ \\ 11 & 8 & 3 & 757.51 & 919.94 & 172.15 & \circ \\ 13 & 8 & 3 & 1266.90 & 1522.48 & 215.19 & \circ \\ 15 & 8 & 3 & 4299.95 & 4077.87 & 247.47 & \circ \\ 17 & 8 & 3 & 2576.39 & 2437.04 & 317.41 & \circ \\ 19 & 8 & 3 & 1024.43 & 1022.16 & 295.89 & \circ \\ 0 & 9 & 3 & 17426.70 & 17150.70 & 683.23 & \circ \\ 2 & 9 & 3 & 4818.96 & 4422.17 & 333.55 & \circ \\ 4 & 9 & 3 & 55.81 & -285.13 & 231.33 & < \\ 6 & 9 & 3 & 1035.82 & 1242.73 & 242.09 & \circ \\ 8 & 9 & 3 & 5040.10 & 5121.54 & 306.65 & \circ \\ 10 & 9 & 3 & 434.95 & 656.33 & 231.33 & < \\ 12 & 9 & 3 & 943.45 & 925.32 & 236.71 & \circ \\ 0 & 0 & 4 & 157861.00 & 168919.00 & 5277.56 & \circ \\ 2 & 0 & 4 & 29109.80 & 26581.50 & 1221.21 & \circ \\ 4 & 0 & 4 & 180.31 & 511.08 & 112.97 & \circ \\ 6 & 0 & 4 & 17405.40 & 20921.90 & 435.76 & \circ \\ 8 & 0 & 4 & 3619.10 & 3765.84 & 102.22 & \circ \\ 10 & 0 & 4 & 5534.71 & 5508.89 & 129.12 & \circ \\ 12 & 0 & 4 & 148.70 & 209.81 & 59.18 & \circ \\ 14 & 0 & 4 & 5967.86 & 6525.66 & 177.53 & \circ \\ 16 & 0 & 4 & 18885.40 & 16548.20 & 392.72 & \circ \\ 18 & 0 & 4 & 185.05 & 150.63 & 80.70 & < \\ 20 & 0 & 4 & 4137.26 & 3970.27 & 129.12 & \circ \\ 22 & 0 & 4 & 244.39 & 398.10 & 86.08 & \circ \\ 24 & 0 & 4 & 10258.50 & 9075.67 & 166.77 & \circ \\ 26 & 0 & 4 & 7854.09 & 7520.92 & 285.13 & \circ \\ 28 & 0 & 4 & 2745.18 & 2716.78 & 204.43 & \circ \\ 30 & 0 & 4 & 3848.05 & 4163.94 & 242.09 & \circ \\ 32 & 0 & 4 & 6229.94 & 7327.25 & 408.86 & \circ \\ 34 & 0 & 4 & 1018.62 & 1893.68 & 451.90 & \circ \\ 1 & 1 & 4 & 238.72 & 166.77 & 75.32 & < \\ 3 & 1 & 4 & 3210.75 & 2878.18 & 96.84 & \circ \\ 5 & 1 & 4 & 11344.40 & 10436.80 & 209.81 & \circ \\ 7 & 1 & 4 & 49766.60 & 49133.50 & 731.65 & \circ \\ 9 & 1 & 4 & 454.54 & 355.07 & 48.42 & \circ \\ 11 & 1 & 4 & 262.28 & 403.48 & 48.42 & \circ \\ 13 & 1 & 4 & 31368.80 & 31374.80 & 451.90 & \circ \\ 15 & 1 & 4 & 12281.10 & 12249.70 & 199.05 & \circ \\ 17 & 1 & 4 & 259.17 & 166.77 & 48.42 & \circ \\ 19 & 1 & 4 & 622.61 & 726.27 & 48.42 & \circ \\ 21 & 1 & 4 & 1430.71 & 1226.59 & 59.18 & \circ \\ 23 & 1 & 4 & 2580.04 & 2786.72 & 80.70 & \circ \\ 25 & 1 & 4 & 1744.18 & 1543.99 & 69.94 & \circ \\ 27 & 1 & 4 & 3884.34 & 4147.80 & 199.05 & \circ \\ 29 & 1 & 4 & 686.70 & 710.13 & 161.39 & \circ \\ 31 & 1 & 4 & 572.72 & 559.50 & 177.53 & \circ \\ & & & & & & \end{array}$




$\begin{array}{rrrrrrl}33 & 1 & 4 & 2027.92 & 2732.92 & 322.79 & \circ \\ 0 & 2 & 4 & 453.00 & 494.94 & 118.36 & \circ \\ 2 & 2 & 4 & 3482.31 & 3055.71 & 166.77 & \circ \\ 4 & 2 & 4 & 9741.66 & 9371.56 & 242.09 & \circ \\ 6 & 2 & 4 & 61319.40 & 66391.80 & 1479.44 & \circ \\ 8 & 2 & 4 & 184289.00 & 195662.00 & 3523.75 & \circ \\ 10 & 2 & 4 & 36543.00 & 35522.60 & 537.98 & \circ \\ 12 & 2 & 4 & 5157.45 & 5261.42 & 96.84 & \circ \\ 14 & 2 & 4 & 89.19 & 166.77 & 48.42 & \circ \\ 16 & 2 & 4 & 17965.50 & 18845.30 & 268.99 & \circ \\ 18 & 2 & 4 & 7836.36 & 8053.52 & 112.97 & \circ \\ 20 & 2 & 4 & 253.60 & 295.89 & 48.42 & \circ \\ 22 & 2 & 4 & 2713.76 & 2781.34 & 69.94 & \circ \\ 24 & 2 & 4 & 8083.96 & 8021.24 & 123.74 & \circ \\ 26 & 2 & 4 & 459.27 & 392.72 & 69.94 & \circ \\ 28 & 2 & 4 & 11649.30 & 12373.50 & 268.99 & \circ \\ 30 & 2 & 4 & 1421.98 & 1484.82 & 204.43 & \circ \\ 32 & 2 & 4 & 2282.75 & 2523.11 & 231.33 & \circ \\ 1 & 3 & 4 & 675.66 & 801.59 & 112.97 & \circ \\ 3 & 3 & 4 & 6882.46 & 9748.15 & 312.03 & \circ \\ 5 & 3 & 4 & 4751.47 & 5508.89 & 177.53 & \circ \\ 7 & 3 & 4 & 12543.60 & 12470.30 & 258.23 & \circ \\ 9 & 3 & 4 & 2671.09 & 2652.23 & 96.84 & \circ \\ 11 & 3 & 4 & 5239.68 & 5186.10 & 107.60 & \circ \\ 13 & 3 & 4 & 14054.80 & 13293.40 & 199.05 & \circ \\ 15 & 3 & 4 & 3799.73 & 3400.02 & 75.32 & \circ \\ 17 & 3 & 4 & 863.36 & 796.21 & 59.18 & \circ \\ 19 & 3 & 4 & 773.46 & 602.53 & 64.56 & \circ \\ 21 & 3 & 4 & 1751.24 & 1581.65 & 75.32 & \circ \\ 23 & 3 & 4 & 2199.67 & 2237.98 & 86.08 & \circ \\ 25 & 3 & 4 & 942.58 & 1032.92 & 80.70 & \circ \\ 27 & 3 & 4 & 2867.79 & 2819.00 & 107.60 & \circ \\ 29 & 3 & 4 & 651.56 & 839.24 & 145.25 & \circ \\ 31 & 3 & 4 & 251.88 & 371.20 & 188.29 & < \\ 0 & 4 & 4 & 128636.00 & 126941.00 & 2835.14 & \circ \\ 2 & 4 & 4 & 27374.10 & 28496.70 & 672.47 & \circ \\ 4 & 4 & 4 & 2312.78 & 3018.05 & 145.25 & \circ \\ 6 & 4 & 4 & 2942.34 & 2878.18 & 107.60 & \circ \\ 8 & 4 & 4 & 2233.30 & 2625.33 & 91.46 & \circ \\ 10 & 4 & 4 & 169.62 & 177.53 & 69.94 & < \\ 12 & 4 & 4 & 514.91 & 500.32 & 69.94 & \circ \\ 14 & 4 & 4 & 7360.82 & 7321.87 & 150.63 & \circ \\ 16 & 4 & 4 & 23239.20 & 22519.70 & 328.17 & \circ \\ 18 & 4 & 4 & 531.91 & 435.76 & 64.56 & \circ \\ 20 & 4 & 4 & 5829.85 & 5750.98 & 118.36 & \circ \\ 22 & 4 & 4 & 115.46 & 263.61 & 69.94 & \circ \\ 24 & 4 & 4 & 8775.91 & 8946.56 & 166.77 & \circ \\ 26 & 4 & 4 & 4194.53 & 3927.23 & 118.36 & \circ \\ 28 & 4 & 4 & 311.41 & 279.75 & 172.15 & < \\ 30 & 4 & 4 & 1399.69 & 1307.28 & 193.67 & \circ \\ 1 & 5 & 4 & 1761.71 & 1823.74 & 80.70 & \circ \\ 3 & 5 & 4 & 3517.62 & 3459.19 & 107.60 & \circ \\ 5 & 5 & 4 & 1620.51 & 1726.91 & 96.84 & \circ \\ 7 & 5 & 4 & 9304.51 & 9118.71 & 166.77 & \circ \\ & & & & & & \end{array}$




\begin{tabular}{|c|c|c|c|c|c|}
\hline 9 & 5 & 4 & 3027.86 & 3405.40 & 102.22 \\
\hline 11 & 5 & 4 & 1963.23 & 1909.82 & 91.46 \\
\hline 13 & 5 & 4 & 3922.40 & 4465.21 & 107.60 \\
\hline 15 & 5 & 4 & 1451.58 & 1366.46 & 86.08 \\
\hline 17 & 5 & 4 & 3216.18 & 3647.49 & 107.60 \\
\hline 19 & 5 & 4 & 2989.65 & 2695.27 & 102.22 \\
\hline 21 & 5 & 4 & 49.55 & 64.56 & 91.46 \\
\hline 23 & 5 & 4 & 904.51 & 909.18 & 112.97 \\
\hline 25 & 5 & 4 & 712.49 & 715.51 & 96.84 \\
\hline 27 & 5 & 4 & 789.57 & 683.23 & 129.12 \\
\hline 0 & 6 & 4 & 2590.75 & 2915.84 & 145.25 \\
\hline 2 & 6 & 4 & 4717.79 & 4562.05 & 118.36 \\
\hline 4 & 6 & 4 & 1000.98 & 1226.59 & 91.46 \\
\hline 6 & 6 & 4 & 9281.53 & 9920.30 & 188.29 \\
\hline 8 & 6 & 4 & 26698.30 & 29405.80 & 446.52 \\
\hline 10 & 6 & 4 & 4736.03 & 4798.76 & 123.74 \\
\hline 12 & 6 & 4 & 1436.66 & 1377.22 & 96.84 \\
\hline 14 & 6 & 4 & 333.26 & 123.74 & 102.22 \\
\hline 16 & 6 & 4 & 2658.72 & 2480.07 & 107.60 \\
\hline 18 & 6 & 4 & 3170.23 & 3631.35 & 134.49 \\
\hline 20 & 6 & 4 & 1219.51 & 1231.97 & 172.15 \\
\hline 22 & 6 & 4 & 2800.08 & 3077.23 & 193.67 \\
\hline 24 & 6 & 4 & 3596.18 & 4196.22 & 215.19 \\
\hline 26 & 6 & 4 & 31.06 & 43.04 & 145.25 \\
\hline 1 & 7 & 4 & 1311.06 & 1275.01 & 139.87 \\
\hline 3 & 7 & 4 & 4913.06 & 5363.63 & 215.19 \\
\hline 5 & 7 & 4 & 1.54 & 48.42 & 150.63 \\
\hline 7 & 7 & 4 & 17.88 & 69.94 & 112.97 \\
\hline 9 & 7 & 4 & 5862.42 & 6251.29 & 204.43 \\
\hline 11 & 7 & 4 & 6818.43 & 7106.68 & 220.57 \\
\hline 13 & 7 & 4 & 158.69 & 145.25 & 134.49 \\
\hline 15 & 7 & 4 & 9.83 & 64.56 & 166.77 \\
\hline 17 & 7 & 4 & 1681.30 & 1716.15 & 188.29 \\
\hline 19 & 7 & 4 & 922.67 & 774.69 & 182.91 \\
\hline 21 & 7 & 4 & 732.53 & 677.85 & 182.91 \\
\hline 23 & 7 & 4 & 1360.44 & 1140.51 & 182.91 \\
\hline 0 & 8 & 4 & 28637.90 & 29642.50 & 839.24 \\
\hline 2 & 8 & 4 & 5456.31 & 5810.15 & 182.91 \\
\hline 4 & 8 & 4 & .52 & 2394. & 139.87 \\
\hline 6 & 8 & 4 & 311.93 & 435.76 & 172.15 \\
\hline 8 & 8 & 4 & 3130.54 & 3362.36 & 204.43 \\
\hline 10 & 8 & 4 & 664.75 & 817.72 & 172.15 \\
\hline 12 & 8 & 4 & 7.78 & 150.63 & 172.15 \\
\hline 14 & 8 & 4 & 4604.84 & 5132.30 & 236.71 \\
\hline 16 & 8 & 4 & 10906.00 & 12169.00 & 376.58 \\
\hline 18 & 8 & 4 & 439.46 & 355.07 & 199.05 \\
\hline 1 & 9 & 4 & 874.69 & 575.64 & 204.43 \\
\hline 3 & 9 & 4 & 1727.77 & 2361.72 & 247.47 \\
\hline 5 & 9 & 4 & 10.35 & -102.22 & 204.43 \\
\hline 7 & 9 & 4 & 207.50 & 290.51 & 209.81 \\
\hline 9 & 9 & 4 & 1759.68 & 1829.12 & 220.57 \\
\hline 11 & 9 & 4 & 1616.66 & 1619.31 & 263.61 \\
\hline 1 & 0 & 5 & 7553.35 & 6800.03 & 193.67 \\
\hline 3 & 0 & 5 & 14597.60 & 13476.30 & 468.04 \\
\hline
\end{tabular}




\begin{tabular}{|c|c|c|c|c|c|}
\hline 5 & $\theta$ & 5 & 73.72 & 172.15 & 123.74 \\
\hline 7 & 0 & 5 & 13176.40 & 15117.20 & 365.82 \\
\hline 9 & 0 & 5 & 632.80 & 521.84 & 75.32 \\
\hline 11 & 0 & 5 & 94.23 & 242.09 & 75.32 \\
\hline 13 & 0 & 5 & 5433.52 & 6240.53 & 182.91 \\
\hline 15 & 0 & 5 & 146.02 & 102.22 & 107.60 \\
\hline 17 & 0 & 5 & 15009.00 & 16602.00 & 468.04 \\
\hline 19 & $\theta$ & 5 & 11811.70 & 12411.10 & 365.82 \\
\hline 21 & 0 & $b$ & 7.16 & 48.42 & 91.46 \\
\hline 23 & 0 & 5 & 1977.73 & 1769.94 & 112.97 \\
\hline 25 & 0 & 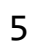 & 702.11 & 699.37 & 86.08 \\
\hline 27 & 0 & & 938.31 & 973.74 & 118.36 \\
\hline 29 & 0 & & 3076.04 & 3071.85 & 220.57 \\
\hline 31 & 0 & & 5194.10 & 5643.38 & 371.20 \\
\hline 33 & 0 & 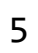 & 338.45 & 882.28 & 295.89 \\
\hline 0 & 1 & 5 & 10650.40 & 8015.86 & 306.65 \\
\hline 2 & 1 & כ & 1783.53 & 2103.49 & 123.74 \\
\hline 4 & 1 & 5 & 25.41 & 80.70 & 69.94 \\
\hline 6 & 1 & 5 & 5565.05 & 5750.98 & 129.12 \\
\hline 8 & 1 & 5 & 34407.20 & 36270.40 & 586.40 \\
\hline 10 & 1 & 5 & 6967.78 & 6907.63 & 145.25 \\
\hline 12 & 1 & 5 & 1251.56 & 1140.51 & 69.94 \\
\hline 14 & 1 & 5 & 337.59 & 139.87 & 64.56 \\
\hline 16 & 1 & 5 & 12221.00 & 11943.10 & 231.33 \\
\hline 18 & 1 & 5 & 1592.55 & 1802.22 & 86.08 \\
\hline 20 & 1 & 5 & 40.97 & 102.22 & 80.70 \\
\hline 22 & 1 & 5 & 1261.46 & 968.36 & 75.32 \\
\hline 24 & 1 & 5 & 5714.46 & 5627.24 & 118.36 \\
\hline 26 & 1 & 5 & 887.78 & 790.83 & 80.70 \\
\hline 28 & 1 & 5 & 652.15 & 543.36 & 91.46 \\
\hline 30 & 1 & 5 & 1.40 & -48.42 & 177.53 \\
\hline 32 & 1 & 5 & 2752.21 & 3157.93 & 231.33 \\
\hline 1 & 2 & 5 & 3980.81 & 4115.52 & 145.25 \\
\hline 3 & 2 & 5 & 921.85 & 1006.02 & 86.08 \\
\hline 5 & 2 & 5 & 10688.70 & 11007.00 & 285.13 \\
\hline 7 & 2 & 5 & 102.82 & 182.91 & 75.32 \\
\hline 9 & 2 & 5 & 116461.00 & 122594.00 & 2055.07 \\
\hline 11 & 2 & 5 & 117609.00 & 119307.00 & 1872.16 \\
\hline 13 & 2 & 5 & 1930.58 & 2307.92 & 75.32 \\
\hline 15 & 2 & 5 & 3864.97 & 4126.28 & 86.08 \\
\hline 17 & 2 & 5 & 133.32 & 145.25 & 64.56 \\
\hline 19 & 2 & 5 & 11.03 & -16.14 & 59.18 \\
\hline 21 & 2 & 5 & 5747.11 & 6084.52 & 112.97 \\
\hline 23 & 2 & 5 & 3059.97 & 2775.96 & 80.70 \\
\hline 25 & 2 & 5 & 3483.57 & 3588.31 & 102.22 \\
\hline 27 & 2 & 5 & 3066.29 & 3109.51 & 123.74 \\
\hline 29 & 2 & 5 & 3476.18 & 3862.68 & 215.19 \\
\hline 31 & 2 & 5 & 3339.47 & 3287.04 & 209.81 \\
\hline 0 & 3 & 5 & 43901.70 & 47885.30 & 979.12 \\
\hline 2 & 3 & 5 & 9259.84 & 9425.36 & 263.61 \\
\hline 4 & 3 & 5 & 270.99 & 328.17 & 107.60 \\
\hline 6 & 3 & 5 & 8317.52 & 7908.26 & 166.77 \\
\hline 8 & 3 & 5 & 37568.60 & 39713.50 & 645.57 \\
\hline 10 & 3 & 5 & 5782.25 & 5745.60 & 123.74 \\
\hline
\end{tabular}




\begin{tabular}{|c|c|c|c|c|c|}
\hline 12 & 3 & 5 & 7258.45 & 7155.10 & 123.74 \\
\hline 14 & 3 & 5 & 38.39 & 96.84 & 53.80 \\
\hline 16 & 3 & 5 & 12739.50 & 11372.80 & 166.77 \\
\hline 18 & 3 & 5 & 889.12 & 946.84 & 59.18 \\
\hline 20 & 3 & 5 & 1182.41 & 1151.27 & 69.94 \\
\hline 22 & 3 & 5 & 556.92 & 645.57 & 69.94 \\
\hline 24 & 3 & 5 & 6573.51 & 6175.98 & 123.74 \\
\hline 26 & 3 & 5 & 1020.82 & 898.42 & 102.22 \\
\hline 28 & 3 & 5 & 1197.99 & 1118.99 & 182.91 \\
\hline 30 & 3 & 5 & 11.96 & 64.56 & 166.77 \\
\hline 1 & 4 & 5 & 9376.59 & 8026.62 & 172.15 \\
\hline 3 & 4 & 5 & 15273.10 & 14154.20 & 365.82 \\
\hline 5 & 4 & 5 & 1474.15 & 1178.17 & 102.22 \\
\hline 7 & 4 & 5 & 12521.80 & 12771.60 & 258.23 \\
\hline 9 & 4 & 5 & 229.61 & 215.19 & 80.70 \\
\hline 11 & 4 & 5 & 79.84 & 102.22 & 75.32 \\
\hline 13 & 4 & 5 & 8058.61 & 7542.44 & 145.25 \\
\hline 15 & 4 & 5 & 978.72 & 1059.82 & 75.32 \\
\hline 17 & 4 & 5 & 11418.80 & 11491.20 & 188.29 \\
\hline 19 & 4 & 5 & 9098.82 & 9506.06 & 150.63 \\
\hline 21 & 4 & 5 & 149.13 & 231.33 & 69.94 \\
\hline 23 & 4 & 5 & 1569.23 & 1791.46 & 86.08 \\
\hline 25 & 4 & 5 & 1585.95 & 1769.94 & 102.22 \\
\hline 27 & 4 & 5 & 2467.24 & 2840.52 & 150.63 \\
\hline 29 & 4 & 5 & 711.14 & 839.24 & 193.67 \\
\hline 0 & 5 & 5 & 637.63 & 882.28 & 112.97 \\
\hline 2 & 5 & 5 & 102.82 & 225.95 & 80.70 \\
\hline 4 & 5 & 5 & 480.01 & 381.96 & 86.08 \\
\hline 6 & 5 & 5 & 1118.12 & 1086.71 & 96.84 \\
\hline 8 & 5 & 5 & 13010.10 & 13492.50 & 242.09 \\
\hline 10 & 5 & 5 & 3164.96 & 2985.77 & 107.60 \\
\hline 12 & 5 & 5 & 11.00 & 107.60 & 86.08 \\
\hline 14 & 5 & 5 & 859.56 & 726.27 & 96.84 \\
\hline 16 & 5 & 5 & 6734.69 & 7052.88 & 134.49 \\
\hline 18 & 5 & 5 & 1606.30 & 1172.79 & 86.08 \\
\hline 20 & 5 & 5 & 194.42 & 182.91 & 80.70 \\
\hline 22 & 5 & 5 & 1310.84 & 1129.75 & 96.84 \\
\hline 24 & 5 & 5 & 3472.07 & 3706.66 & 129.12 \\
\hline 26 & 5 & 5 & 587.88 & 667.09 & 145.25 \\
\hline 1 & 6 & 5 & 33.01 & -16.14 & 96.84 \\
\hline 3 & 6 & 5 & 1828.00 & 1850.64 & 139.87 \\
\hline 5 & 6 & 5 & 11343.70 & 11039.30 & 252.85 \\
\hline 7 & 6 & 5 & 3478.40 & 3728.18 & 129.12 \\
\hline 9 & 6 & 5 & 8606.63 & 8075.04 & 193.67 \\
\hline 11 & 6 & 5 & 8797.66 & 9048.78 & 215.19 \\
\hline 13 & 6 & 5 & 1287.59 & 1280.39 & 112.97 \\
\hline 15 & 6 & 5 & 8569.46 & 9048.78 & 236.71 \\
\hline 17 & 6 & 5 & 2748.82 & 2636.09 & 134.49 \\
\hline 19 & 6 & 5 & 1359.56 & 1387.98 & 139.87 \\
\hline 21 & 6 & 5 & 2339.80 & 2797.48 & 177.53 \\
\hline 23 & 6 & 5 & 606.86 & 828.48 & 156.01 \\
\hline 25 & 6 & 5 & 1320.40 & 1404.12 & 156.01 \\
\hline 0 & 7 & 5 & 11333.00 & 12039.90 & 505.70 \\
\hline 2 & 7 & 5 & 3079.66 & 2700.65 & 161.39 \\
\hline
\end{tabular}




\begin{tabular}{|c|c|c|c|c|c|}
\hline 4 & 7 & 5 & 6.94 & 123.74 & 107.60 \\
\hline 6 & 7 & 5 & 1558.04 & 1167.41 & 123.74 \\
\hline 8 & 7 & 5 & 6518.33 & 7144.34 & 204.43 \\
\hline 10 & 7 & 5 & 684.37 & 677.85 & 112.97 \\
\hline 12 & 7 & 5 & 1748.03 & 1856.02 & 139.87 \\
\hline 14 & 7 & 5 & 0.48 & -134.49 & 156.01 \\
\hline 16 & 7 & 5 & 2876.06 & 3179.44 & 172.15 \\
\hline 18 & 7 & 5 & 495.29 & 887.66 & 172.15 \\
\hline 20 & 7 & 5 & 94.42 & 32.28 & 166.77 \\
\hline 1 & 8 & 5 & 3360.77 & 3028.81 & 182.910 \\
\hline 3 & 8 & 5 & 5405.89 & 5826.29 & 285.13 \\
\hline 5 & 8 & 5 & 920.40 & 919.94 & 156.010 \\
\hline 7 & 8 & 5 & 4884.71 & 4599.70 & 204.43 \\
\hline 9 & 8 & 5 & 77.61 & 96.84 & 161.39 \\
\hline 11 & 8 & 5 & 82.06 & -43.04 & 150.63 \\
\hline 13 & 8 & 5 & 4703.67 & 4319.96 & 188.29 \\
\hline 15 & 8 & 5 & 1329.97 & 1328.80 & 204.43 \\
\hline 0 & 9 & 5 & 629.75 & 833.86 & 306.65 \\
\hline 2 & 9 & 5 & 43.33 & -64.56 & 225.95 \\
\hline 4 & 9 & 5 & 672.21 & 763.93 & 215.19 \\
\hline 6 & 9 & 5 & 501.92 & 419.62 & 215.19 \\
\hline 8 & 9 & 5 & 5247.59 & 5169.96 & 279.75 \\
\hline 0 & 0 & 6 & 19154.60 & 18893.80 & 645.57 \\
\hline 2 & 0 & 6 & 7335.40 & 8101.94 & 322.79 \\
\hline 4 & 0 & 6 & 1860.90 & 2173.43 & 172.15 \\
\hline 6 & 0 & 6 & 19135.10 & 20938.10 & 500.32 \\
\hline 8 & $\theta$ & 6 & 70216.10 & 73315.50 & 1468.68 \\
\hline 10 & $\theta$ & 6 & 16569.80 & 18441.90 & 398.10 \\
\hline 12 & 0 & 6 & 615.69 & 683.23 & 91.46 \\
\hline 14 & 0 & 6 & 894.20 & 979.12 & 123.74 \\
\hline 16 & 0 & 6 & 6456.33 & 7241.17 & 215.19 \\
\hline 18 & 0 & 6 & 8802.24 & 8623.77 & 285.13 \\
\hline 20 & 0 & 6 & 5107.36 & 5325.97 & 215.19 \\
\hline 22 & 0 & 6 & 6236.50 & 6364.27 & 209.81 \\
\hline 24 & 0 & 6 & 5799.57 & 5847.81 & 231.33 \\
\hline 26 & 0 & 6 & 274.56 & 279.75 & 188.29 \\
\hline 28 & 0 & 6 & 2198.48 & 1925.96 & 193.67 \\
\hline 30 & 0 & 6 & 9.19 & 328.17 & 274.37 \\
\hline 32 & 0 & 6 & 1714.61 & 2114.25 & 322.79 \\
\hline 1 & 1 & 6 & 2081.32 & 2490.83 & 123.74 \\
\hline 3 & 1 & 6 & 865.16 & 1151.27 & 91.460 \\
\hline 5 & 1 & 6 & 21163.10 & 24230.50 & 430.380 \\
\hline 7 & 1 & 6 & 25879.50 & 26506.10 & 414.24 \\
\hline 9 & 1 & 6 & 1840.62 & 1925.96 & 75.32 \\
\hline 11 & 1 & 6 & 2347.33 & 2480.07 & 80.70 \\
\hline 13 & 1 & 6 & 17812.30 & 17392.80 & 268.99 \\
\hline 15 & 1 & 6 & 14424.90 & 14450.10 & 231.33 \\
\hline 17 & 1 & 6 & 22.45 & 53.80 & 69.94 \\
\hline 19 & 1 & 6 & 93.34 & 96.84 & 69.94 \\
\hline 21 & 1 & 6 & 3769.49 & 3577.55 & 112.97 \\
\hline 23 & 1 & 6 & 3002.53 & 2856.66 & 112.97 \\
\hline 25 & 1 & 6 & 2204.58 & 2055.07 & 123.74 \\
\hline 27 & 1 & 6 & 4137.98 & 4631.98 & 225.95 \\
\hline 29 & 1 & 6 & 653.09 & 537.98 & 204.43 \\
\hline
\end{tabular}




\begin{tabular}{|c|c|c|c|c|c|}
\hline 31 & 1 & 6 & 794.62 & 624.05 & 204.43 \\
\hline 0 & 2 & 6 & 83255.70 & 86926.40 & 1947.48 \\
\hline 2 & 2 & 6 & 45118.80 & 45421.40 & 925.32 \\
\hline 4 & 2 & 6 & 952.41 & 1113.61 & 86.08 \\
\hline 6 & 2 & 6 & 7132.44 & 7676.93 & 156.01 \\
\hline 8 & & 6 & 1107.22 & 1140.51 & 69.94 \\
\hline 10 & & 6 & 886.78 & 645.57 & 69.94 \\
\hline 12 & 2 & 6 & 1177.64 & 1178.17 & 69.94 \\
\hline 14 & 2 & 6 & 9290.51 & 9468.40 & 156.01 \\
\hline 16 & 2 & 6 & 11357.90 & 11432.00 & 182.91 \\
\hline 18 & 2 & 6 & 1101.72 & 876.90 & 69.94 \\
\hline 20 & 2 & 6 & 1930.35 & 1683.87 & 75.32 \\
\hline 22 & 2 & 6 & 49.22 & 21.52 & 64.56 \\
\hline 24 & 2 & 6 & 5434.18 & 5288.32 & 102.22 \\
\hline 26 & 2 & 6 & 8226.38 & 7542.44 & 161.39 \\
\hline 28 & 2 & 6 & 1801.62 & 1899.06 & 193.67 \\
\hline 30 & 2 & 6 & 3092.33 & 3071.85 & 215.19 \\
\hline 1 & 3 & 6 & 5304.38 & 6046.86 & 172.15 \\
\hline 3 & 3 & 6 & 9441.26 & 8155.73 & 209.81 \\
\hline 5 & 3 & 6 & 23013.00 & 22535.90 & 441.14 \\
\hline 7 & 3 & 6 & 20619.60 & 20878.90 & 355.07 \\
\hline 9 & 3 & 6 & 9117.91 & 9409.22 & 156.01 \\
\hline 11 & 3 & 6 & 12439.30 & 12798.50 & 204.43 \\
\hline 13 & 3 & 6 & 11103.60 & 11453.50 & 172.15 \\
\hline 15 & 3 & 6 & 5938.96 & 6003.82 & 112.97 \\
\hline 17 & 3 & 6 & 5312.33 & 5417.43 & 107.60 \\
\hline 19 & 3 & 6 & 7938.03 & 8015.86 & 129.12 \\
\hline 21 & 3 & 6 & 1035.55 & 1022.16 & 69.94 \\
\hline 23 & 3 & 6 & 916.34 & 962.98 & 80.70 \\
\hline 25 & 3 & 6 & 5177.05 & 4960.15 & 129.12 \\
\hline 27 & 3 & 6 & 5731.52 & 6310.47 & 199.05 \\
\hline 29 & 3 & 6 & 1491.17 & 1135.13 & 193.67 \\
\hline 0 & 4 & 6 & 4872.26 & 5481.99 & 204.43 \\
\hline 2 & 4 & 6 & 2086.99 & 2259.50 & 129.12 \\
\hline 4 & 4 & 6 & 499.28 & 344.30 & 123.74 \\
\hline 6 & 4 & 6 & 12168.90 & 12572.50 & 258.23 \\
\hline 8 & 4 & 6 & 35964.30 & 36808.40 & 656.33 \\
\hline 10 & 4 & 6 & 8091.49 & 8451.62 & 182.91 \\
\hline 12 & 4 & 6 & 1286.31 & 1350.32 & 91.46 \\
\hline 14 & 4 & 6 & 5.90 & 59.18 & 69.94 \\
\hline 16 & 4 & 6 & 2422.77 & 2636.09 & 91.46 \\
\hline 18 & 4 & 6 & 3798.59 & 3916.47 & 102.22 \\
\hline 20 & 4 & 6 & 1756.75 & 1818.36 & 80.70 \\
\hline 22 & 4 & 6 & 2576.30 & 2533.87 & 96.84 \\
\hline 24 & 4 & 6 & 2774.99 & 3147.17 & 118.36 \\
\hline 26 & 4 & 6 & 102.12 & 172.15 & 134.49 \\
\hline 28 & 4 & 6 & 1450.50 & 1457.92 & 193.67 \\
\hline 1 & 5 & 6 & 1995.21 & 1856.02 & 107.60 \\
\hline 3 & 5 & 6 & 1336.95 & 1705.39 & 134.49 \\
\hline 5 & 5 & 6 & 2093.30 & 2114.25 & 102.22 \\
\hline 7 & 5 & 6 & 2474.64 & 2743.68 & 118.36 \\
\hline 9 & 5 & 6 & 5307.17 & 4997.81 & 145.25 \\
\hline 11 & 5 & 6 & 4640.86 & 4820.27 & 145.25 \\
\hline 13 & 5 & 6 & 1830.41 & 1640.83 & 112.97 \\
\hline
\end{tabular}




\begin{tabular}{|c|c|c|c|c|c|}
\hline 15 & & 6 & 3142.08 & 3023.43 & 107.60 \\
\hline 17 & & 6 & 122.04 & 37.66 & 91.46 \\
\hline 19 & J & 6 & 57.27 & -5.38 & 86.08 \\
\hline 21 & J & 6 & 2915.08 & 2819.00 & 123.74 \\
\hline 23 & 5 & 6 & 2912.58 & 3265.52 & 129.12 \\
\hline 25 & 5 & 6 & 216.32 & 322.79 & 129.12 \\
\hline$\theta$ & 6 & 6 & 38629.90 & 37254.90 & 995.26 \\
\hline 2 & 6 & 6 & 19672.30 & 20141.90 & 430.38 \\
\hline 4 & 6 & 6 & 39.80 & -172.15 & 134.49 \\
\hline 6 & 6 & 6 & 513.09 & 564.88 & 134.49 \\
\hline 8 & & 6 & 74.69 & 150.63 & 150.63 \\
\hline 10 & 0 & 6 & 106.14 & 166.77 & 129.12 \\
\hline 12 & 6 & 6 & 176.46 & 102.22 & 145.25 \\
\hline 14 & 6 & 6 & 5962.04 & 6353.51 & 220.57 \\
\hline 16 & 6 & 6 & 5648.65 & 5971.55 & 177.53 \\
\hline 18 & 6 & 6 & 145.01 & 511.08 & 123.74 \\
\hline 20 & 6 & 6 & 1903.15 & 1969.00 & 139.87 \\
\hline 22 & 6 & 6 & 332.21 & 392.72 & 134.49 \\
\hline 1 & 7 & 6 & 1647.07 & 1839.88 & 112.97 \\
\hline 3 & 7 & 6 & 3103.15 & 3044.95 & 150.63 \\
\hline 5 & 7 & 6 & 2423.36 & 2469.32 & 139.87 \\
\hline 7 & 7 & 6 & 1850.66 & 2324.06 & 139.87 \\
\hline 9 & 7 & 6 & 3822.36 & 3991.79 & 166.77 \\
\hline 11 & 7 & 6 & 5481.71 & 5627.24 & 209.81 \\
\hline 13 & 7 & 6 & 517.19 & 683.23 & 161.39 \\
\hline 15 & 7 & 6 & 180.31 & 295.89 & 145.25 \\
\hline 17 & 7 & 6 & 3523.77 & 3744.32 & 209.81 \\
\hline 19 & 7 & 6 & 4509.65 & 4508.25 & 220.57 \\
\hline 0 & 8 & 6 & 213.43 & 43.04 & 268.99 \\
\hline 2 & 8 & 6 & 215.20 & 478.80 & 204.43 \\
\hline 4 & 8 & 6 & 331.90 & 403.48 & 215.19 \\
\hline 6 & 8 & 6 & 5444.17 & 5750.98 & 268.99 \\
\hline 8 & 8 & 6 & 9275.79 & 9850.36 & 290.51 \\
\hline 10 & 8 & 6 & 1422.96 & 1554.75 & 177.53 \\
\hline 12 & 8 & 6 & 1139.16 & 1065.19 & 215.19 \\
\hline 14 & 8 & 6 & 318.82 & 570.26 & 204.43 \\
\hline 1 & 0 & 7 & 2626.54 & 2022.79 & 102.22 \\
\hline 3 & 0 & 7 & 695.21 & 785.45 & 129.12 \\
\hline 5 & 0 & 7 & 5287.19 & 5153.82 & 182.91 \\
\hline 7 & 0 & 7 & 575.31 & 505.70 & 96.84 \\
\hline 9 & 0 & 7 & 44202.50 & 43802.10 & 898.42 \\
\hline 11 & 0 & 7 & 34683.30 & 36308.10 & 753.17 \\
\hline 13 & 0 & 7 & 2.27 & -139.87 & 123.74 \\
\hline 15 & 0 & 7 & 5377.77 & 5180.72 & 209.81 \\
\hline 17 & 0 & 7 & 14.67 & 172.15 & 129.12 \\
\hline 19 & 0 & 7 & 46.87 & 139.87 & 134.49 \\
\hline 21 & 0 & 7 & 5398.52 & 5067.74 & 215.19 \\
\hline 23 & 0 & 7 & 5097.52 & 5083.88 & 247.47 \\
\hline 25 & 0 & 7 & 1111.39 & 936.08 & 150.63 \\
\hline 27 & 0 & 7 & 827.31 & 618.67 & 204.43 \\
\hline 29 & 0 & 7 & 2584.68 & 3324.70 & 328.17 \\
\hline 0 & 1 & 7 & 24266.20 & 26350.10 & 624.05 \\
\hline 2 & 1 & 7 & 9320.14 & 9904.16 & 242.09 \\
\hline 4 & 1 & 7 & 6740.25 & 6595.60 & 182.91 \\
\hline
\end{tabular}




\begin{tabular}{|c|c|c|c|c|c|}
\hline 6 & 1 & 7 & 6917.98 & 7192.75 & 156.01 \\
\hline 8 & 1 & 7 & 28018.60 & 28539.70 & 425.00 \\
\hline 10 & 1 & f & 4200.88 & 4094.01 & 102.22 \\
\hline 12 & 1 & 7 & 22.13 & 48.42 & 69.94 \\
\hline 14 & 1 & I & 405.53 & 457.28 & 75.32 \\
\hline 16 & 1 & & 3746.96 & 3163.31 & 91.46 \\
\hline 18 & 1 & & 43.34 & 59.18 & 75.32 \\
\hline 20 & 1 & & 25.41 & -48.42 & 91.46 \\
\hline 22 & 1 & & 23.02 & 145.25 & 91.46 \\
\hline 24 & 1 & 7 & 716.64 & 693.99 & 91.46 \\
\hline 26 & 1 & 7 & 44.51 & 64.56 & 107.60 \\
\hline 28 & 1 & 7 & 547.33 & 371.20 & 139.87 \\
\hline 30 & 1 & 7 & 58.07 & 349.68 & 193.67 \\
\hline 1 & 2 & 7 & 19363.10 & 19033.60 & 333.55 \\
\hline 3 & 2 & 7 & 43664.10 & 43920.50 & 823.10 \\
\hline 5 & 2 & 7 & 862.42 & 962.98 & 91.46 \\
\hline 7 & 2 & 7 & 13339.10 & 13271.90 & 231.33 \\
\hline 9 & 2 & 7 & 795.51 & 683.23 & 75.32 \\
\hline 11 & 2 & 7 & 520.79 & 688.61 & 75.32 \\
\hline 13 & 2 & 7 & 10296.30 & 10721.90 & 193.67 \\
\hline 15 & 2 & 7 & 469.09 & 543.36 & 69.94 \\
\hline 17 & 2 & 7 & 26411.60 & 26366.30 & 398.10 \\
\hline 19 & 2 & 7 & 26435.60 & 26941.90 & 387.34 \\
\hline 21 & 2 & 7 & 27.45 & -10.76 & 75.32 \\
\hline 23 & 2 & 7 & 3635.85 & 3523.75 & 96.84 \\
\hline 25 & 2 & 7 & 1390.03 & 1619.31 & 107.60 \\
\hline 27 & 2 & 7 & 2182.35 & 2324.06 & 150.63 \\
\hline 29 & 2 & 7 & 2104.45 & 2087.35 & 215.19 \\
\hline 0 & 3 & 7 & 43.82 & 5.38 & 112.97 \\
\hline 2 & 3 & 7 & 19.52 & 172.15 & 102.22 \\
\hline 4 & 3 & 7 & 642.29 & 667.09 & 123.74 \\
\hline 6 & 3 & 7 & 833.11 & 1075.95 & 91.46 \\
\hline 8 & 3 & 7 & 9024.26 & 8360.16 & 177.53 \\
\hline 10 & 3 & 7 & 2864.95 & 3028.81 & 102.22 \\
\hline 12 & 3 & 7 & 839.76 & 806.97 & 75.32 \\
\hline 14 & 3 & 7 & 981.18 & 844.62 & 75.32 \\
\hline 16 & 3 & 7 & 2183.35 & 2356.34 & 86.08 \\
\hline 18 & 3 & 7 & 809.04 & 925.32 & 75.32 \\
\hline 20 & 3 & 7 & 969.90 & 989.88 & 86.08 \\
\hline 22 & 3 & 7 & 144.93 & 166.77 & 86.08 \\
\hline 24 & 3 & 7 & 548.96 & 640.19 & 96.84 \\
\hline 26 & 3 & 7 & 268.53 & 102.22 & 134.49 \\
\hline 28 & 3 & 7 & 26.55 & 268.99 & 188.29 \\
\hline 1 & 4 & 7 & 2598.17 & 2770.58 & 118.36 \\
\hline 3 & 4 & 7 & 675.18 & 1102.85 & 150.63 \\
\hline 5 & 4 & 7 & 9769.59 & 10539.00 & 236.71 \\
\hline 7 & 4 & 7 & 3304.38 & 3421.53 & 129.12 \\
\hline 9 & 4 & 7 & 21856.60 & 20965.00 & 355.07 \\
\hline 11 & 4 & 7 & 19184.10 & 18646.30 & 285.13 \\
\hline 13 & 4 & 7 & 1137.92 & 1118.99 & 86.08 \\
\hline 15 & 4 & 7 & 7182.22 & 6633.26 & 139.87 \\
\hline 17 & 4 & 7 & 8.22 & 26.90 & 80.70 \\
\hline 19 & 4 & 7 & 95.53 & 107.60 & 80.70 \\
\hline 21 & 4 & 7 & 3507.07 & 3464.57 & 112.97 \\
\hline
\end{tabular}




$\begin{array}{rrrrrrr}23 & 4 & 7 & 2741.46 & 2867.42 & 118.36 & \circ \\ 25 & 4 & 7 & 1805.32 & 1764.56 & 145.25 & \circ \\ 27 & 4 & 7 & 1439.95 & 1371.84 & 150.63 & \circ \\ 0 & 5 & 7 & 14453.10 & 15493.70 & 591.78 & \circ \\ 2 & 5 & 7 & 3919.57 & 3991.79 & 215.19 & \circ \\ 4 & 5 & 7 & 1259.87 & 1070.57 & 172.15 & \circ \\ 6 & 5 & 7 & 1360.23 & 1070.57 & 177.53 & \circ \\ 8 & 5 & 7 & 8227.90 & 9387.70 & 441.14 & \circ \\ 10 & 5 & 7 & 616.48 & 650.95 & 123.74 & \circ \\ 12 & 5 & 7 & 299.97 & 381.96 & 123.74 & \circ \\ 14 & 5 & 7 & 160.81 & -5.38 & 112.97 & < \\ 16 & 5 & 7 & 3359.82 & 3136.41 & 145.25 & \circ \\ 18 & 5 & 7 & 430.58 & 532.60 & 112.97 & \circ \\ 20 & 5 & 7 & 211.35 & 215.19 & 112.97 & < \\ 22 & 5 & 7 & 278.52 & 435.76 & 112.97 & \circ \\ 24 & 5 & 7 & 2101.42 & 2125.01 & 123.74 & \circ \\ 1 & 6 & 7 & 5157.17 & 4809.52 & 161.39 & \circ \\ 3 & 6 & 7 & 10215.40 & 8914.28 & 242.09 & \circ \\ 5 & 6 & 7 & 252.65 & 403.48 & 112.97 & \circ \\ 7 & 6 & 7 & 6225.09 & 6520.28 & 220.57 & \circ \\ 9 & 6 & 7 & 788.62 & 645.57 & 199.05 & \circ \\ 11 & 6 & 7 & 1360.10 & 1732.29 & 225.95 & \circ \\ 13 & 6 & 7 & 6852.80 & 6923.77 & 209.81 & \circ \\ 15 & 6 & 7 & 988.32 & 850.00 & 139.87 & \circ \\ 17 & 6 & 7 & 5021.63 & 4997.81 & 177.53 & \circ \\ 19 & 6 & 7 & 4564.36 & 4605.08 & 161.39 & \circ \\ 21 & 6 & 7 & 369.41 & 387.34 & 129.12 & < \\ 0 & 7 & 7 & 565.70 & 462.66 & 242.09 & < \\ 2 & 7 & 7 & 1008.06 & 365.82 & 150.63 & < \\ 4 & 7 & 7 & 555.86 & 355.07 & 156.01 & < \\ 6 & 7 & 7 & 45.68 & 134.49 & 134.49 & < \\ 8 & 7 & 7 & 3259.70 & 3184.82 & 166.77 & \circ \\ 10 & 7 & 7 & 1750.15 & 1764.56 & 145.25 & \circ \\ 12 & 7 & 7 & 834.03 & 806.97 & 156.01 & \circ \\ 14 & 7 & 7 & 1759.12 & 1743.05 & 252.85 & \circ \\ 16 & 7 & 7 & 3300.25 & 3039.57 & 209.81 & \circ \\ 1 & 8 & 7 & 686.95 & 1113.61 & 209.81 & \circ \\ 3 & 8 & 7 & 23.70 & -53.80 & 225.95 & < \\ 5 & 8 & 7 & 6546.86 & 6746.23 & 290.51 & \circ \\ 7 & 8 & 7 & 3377.22 & 3276.28 & 242.09 & \circ \\ 9 & 8 & 7 & 3131.75 & 2802.86 & 242.09 & \circ \\ 11 & 8 & 7 & 3380.77 & 3200.96 & 247.47 & \circ \\ 0 & 0 & 8 & 29285.70 & 28270.70 & 774.69 & \circ \\ 2 & 0 & 8 & 23593.90 & 23353.60 & 650.95 & \circ \\ 4 & 0 & 8 & 141.99 & -26.90 & 139.87 & < \\ 6 & 0 & 8 & 614.14 & 1006.02 & 102.22 & \circ \\ 8 & 0 & 8 & 29.35 & 220.57 & 107.60 & < \\ 10 & 0 & 8 & 87.17 & 177.53 & 107.60 & < \\ 12 & 0 & 8 & 428.27 & 295.89 & 139.87 & < \\ 14 & 0 & 8 & 6678.30 & 6708.57 & 252.85 & \circ \\ 16 & 0 & 8 & 5403.11 & 6348.13 & 247.47 & \circ \\ 18 & 0 & 8 & 2678.00 & 2662.99 & 182.91 & \circ \\ 20 & 0 & 8 & 5.09 & 263.61 & 172.15 & < \\ 22 & 0 & 8 & 750.68 & 505.70 & 172.15 & < \\ & & & & & & \end{array}$




\begin{tabular}{|c|c|c|c|c|c|}
\hline 24 & $\theta$ & 8 & 4009.25 & 3781.98 & 231.33 \\
\hline 26 & 0 & 8 & 6118.03 & 5777.87 & 317.41 \\
\hline 28 & 0 & 8 & 940.63 & 1361.08 & 392.72 \\
\hline 1 & 1 & 8 & 7041.12 & 6869.97 & 145.25 \\
\hline 3 & 1 & 8 & 5479.24 & 5433.57 & 156.01 \\
\hline 5 & 1 & 8 & 10435.40 & 10253.80 & 236.71 \\
\hline 7 & 1 & 8 & 10406.40 & 10781.10 & 209.81 \\
\hline 9 & 1 & 8 & 4475.31 & 4287.68 & 118.36 \\
\hline 11 & 1 & 8 & 5611.86 & 5535.78 & 134.49 \\
\hline 13 & 1 & 8 & 6395.34 & 6504.14 & 156.01 \\
\hline 15 & 1 & 8 & 7519.66 & 7806.05 & 161.39 \\
\hline 17 & 1 & 8 & 2632.15 & 2851.28 & 96.84 \\
\hline 19 & 1 & 8 & 3972.97 & 4206.98 & 129.12 \\
\hline 21 & 1 & 8 & 2490.79 & 2329.44 & 118.36 \\
\hline 23 & 1 & 8 & 2094.31 & 2194.95 & 123.74 \\
\hline 25 & 1 & 8 & 2911.71 & 2706.02 & 123.74 \\
\hline 27 & 1 & 8 & 3164.74 & 2754.44 & 204.43 \\
\hline 0 & 2 & 8 & 56772.10 & 58241.40 & 1328.80 \\
\hline 2 & 2 & 8 & 15881.10 & 16682.70 & 381.96 \\
\hline 4 & 2 & 8 & 79.57 & -59.18 & 102.22 \\
\hline 6 & 2 & 8 & 10459.80 & 10280.70 & 193.67 \\
\hline 8 & 2 & 8 & 16699.60 & 18678.60 & 312.03 \\
\hline 10 & 2 & 8 & 8748.10 & 8639.91 & 172.15 \\
\hline 12 & 2 & 8 & 11.88 & 64.56 & 86.08 \\
\hline 14 & 2 & 8 & 37.02 & 86.08 & 86.08 \\
\hline 16 & 2 & 8 & 39.26 & 139.87 & 80.70 \\
\hline 18 & 2 & 8 & 6771.48 & 6202.88 & 134.49 \\
\hline 20 & 2 & 8 & 13760.40 & 13519.40 & 242.09 \\
\hline 22 & 2 & 8 & 9152.72 & 8306.37 & 161.39 \\
\hline 24 & 2 & 8 & 2429.78 & 2270.26 & 129.12 \\
\hline 26 & 2 & 8 & 730.43 & 957.60 & 145.25 \\
\hline 28 & 2 & 8 & 97.15 & 258.23 & 268.99 \\
\hline 1 & 3 & 8 & 5918.80 & 6310.47 & 166.77 \\
\hline 3 & 3 & 8 & 6887.87 & 7117.44 & 236.71 \\
\hline 5 & 3 & 8 & 5967.50 & 6181.36 & 166.77 \\
\hline 7 & 3 & 8 & 3664.38 & 4691.16 & 129.12 \\
\hline 9 & 3 & 8 & 5956.68 & 6202.88 & 145.25 \\
\hline 11 & 3 & 8 & 9350.29 & 9220.93 & 182.91 \\
\hline 13 & 3 & 8 & 4192.78 & 4260.78 & 123.74 \\
\hline 15 & 3 & 8 & 6620.55 & 6375.03 & 129.12 \\
\hline 17 & 3 & 8 & 615.86 & 527.22 & 86.08 \\
\hline 19 & 3 & 8 & 1014.87 & 919.94 & 86.08 \\
\hline 21 & 3 & 8 & 5580.38 & 5374.39 & 145.25 \\
\hline 23 & 3 & 8 & 4897.21 & 4895.59 & 145.25 \\
\hline 25 & 3 & 8 & 627.82 & 785.45 & 145.25 \\
\hline 27 & 3 & 8 & 1855.77 & 2125.01 & 177.53 \\
\hline 0 & 4 & 8 & 14354.00 & 15789.60 & 489.56 \\
\hline 2 & 4 & 8 & 15223.10 & 15332.30 & 414.24 \\
\hline 4 & 4 & 8 & 662.99 & 376.58 & 225.95 \\
\hline 6 & 4 & 8 & 217.03 & 371.20 & 156.01 \\
\hline 8 & 4 & 8 & 559.70 & 333.55 & 134.49 \\
\hline 10 & 4 & 8 & 103.04 & 252.85 & 123.74 \\
\hline 12 & 4 & 8 & 130.60 & 247.47 & 107.60 \\
\hline 14 & 4 & 8 & 3472.22 & 3771.22 & 145.25 \\
\hline
\end{tabular}




\begin{tabular}{|c|c|c|c|c|c|}
\hline 16 & 4 & 8 & 1031.47 & 1328.80 & 134.49 \\
\hline 18 & 4 & 8 & 566.88 & 613.29 & 129.12 \\
\hline 20 & 4 & 8 & 9.38 & 145.25 & 112.97 \\
\hline 22 & 4 & 8 & 20.90 & -69.94 & 118.36 \\
\hline 24 & 4 & 8 & 720.13 & 839.24 & 123.74 \\
\hline 1 & 5 & 8 & 3501.38 & 3351.60 & 156.01 \\
\hline 3 & 5 & 8 & 2526.24 & 2447.80 & 177.53 \\
\hline 5 & 5 & 8 & 4786.23 & 4798.76 & 177.53 \\
\hline 7 & 5 & 8 & 5723.03 & 5966.17 & 247.47 \\
\hline 9 & כ & 8 & 2244.80 & 2237.98 & 188.29 \\
\hline 11 & 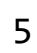 & 8 & 2295.42 & 2291.78 & 172.15 \\
\hline 13 & 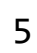 & 8 & 2411.96 & 2738.30 & 145.25 \\
\hline 15 & 5 & 8 & 2331.83 & 2727.54 & 145.25 \\
\hline 17 & 5 & 8 & 3199.60 & 3356.98 & 156.01 \\
\hline 19 & 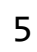 & 8 & 4207.07 & 3948.75 & 166.77 \\
\hline 21 & 5 & 8 & 259.10 & 365.82 & 123.74 \\
\hline 23 & 5 & 8 & 403.81 & 532.60 & 123.74 \\
\hline 0 & 6 & 8 & 720.73 & 914.56 & 182.91 \\
\hline 2 & 6 & 8 & 13.84 & -177.53 & 129.12 \\
\hline 4 & 6 & 8 & 1457.36 & 1441.78 & 139.87 \\
\hline 6 & 6 & 8 & 13293.00 & 13331.10 & 338.93 \\
\hline 8 & 6 & 8 & 18006.20 & 18877.60 & 500.32 \\
\hline 10 & 6 & 8 & 4792.84 & 4352.24 & 236.71 \\
\hline 12 & 6 & 8 & 653.87 & 543.36 & 150.63 \\
\hline 14 & 6 & 8 & 466.14 & 624.05 & 139.87 \\
\hline 16 & 6 & 8 & 3.60 & 193.67 & 139.87 \\
\hline 18 & 6 & 8 & 870.69 & 688.61 & 145.25 \\
\hline 1 & 7 & 8 & 2182.90 & 1829.12 & 215.19 \\
\hline 3 & 7 & 8 & 3363.29 & 3077.23 & 252.85 \\
\hline 5 & 7 & 8 & 661.85 & 704.75 & 199.05 \\
\hline 7 & 7 & 8 & 104.05 & 199.05 & 172.15 \\
\hline 9 & 7 & 8 & 5737.85 & 5406.67 & 263.61 \\
\hline 11 & 7 & 8 & 6924.88 & 7203.51 & 349.68 \\
\hline 13 & 7 & 8 & 171.10 & 199.05 & 252.85 \\
\hline 0 & 8 & 8 & 4437.59 & 4362.99 & 376.58 \\
\hline 2 & 8 & 8 & 5031.53 & 4987.05 & 312.03 \\
\hline 4 & 8 & 8 & 1262.73 & 1054.44 & 225.95 \\
\hline 1 & 0 & 9 & 18148.30 & 18049.10 & 376.58 \\
\hline 3 & 0 & 9 & 21297.10 & 21927.90 & 624.05 \\
\hline 5 & 0 & 9 & 2624.59 & 2840.52 & 134.49 \\
\hline 7 & 0 & 9 & 13239.40 & 12314.30 & 279.75 \\
\hline 9 & 0 & 9 & 500.01 & 381.96 & 134.49 \\
\hline 11 & 0 & 9 & 39.44 & 129.12 & 134.49 \\
\hline 13 & 0 & 9 & 7527.52 & 7300.35 & 279.75 \\
\hline 15 & 0 & 9 & 1768.31 & 2329.44 & 225.95 \\
\hline 17 & 0 & 9 & 17523.00 & 16940.90 & 624.05 \\
\hline 19 & 0 & 9 & 17642.40 & 16790.30 & 618.67 \\
\hline 21 & 0 & 9 & 139.22 & 220.57 & 199.05 \\
\hline 23 & 0 & 9 & 2443.38 & 2324.06 & 225.95 \\
\hline 25 & 0 & 9 & 2748.60 & 2334.82 & 312.03 \\
\hline 27 & 0 & 9 & 2530.87 & 2679.13 & 274.37 \\
\hline 0 & 1 & 9 & 6563.43 & 6068.38 & 215.19 \\
\hline 2 & 1 & 9 & 7331.13 & 7241.17 & 193.67 \\
\hline 4 & 1 & 9 & 11286.40 & 10087.10 & 263.61 \\
\hline
\end{tabular}




\begin{tabular}{|c|c|c|c|c|c|}
\hline 6 & 1 & 9 & 2688.24 & 3297.80 & 118.36 \\
\hline 8 & 1 & 9 & 8193.58 & 7300.35 & 161.39 \\
\hline 10 & 1 & 9 & 1920.19 & 2184.19 & 102.22 \\
\hline 12 & 1 & 9 & 1551.27 & 1339.56 & 102.22 \\
\hline 14 & 1 & 9 & 259.16 & 177.53 & 96.84 \\
\hline 16 & 1 & 9 & 196.00 & 225.95 & 91.46 \\
\hline 18 & 1 & 9 & 0.40 & -150.63 & 139.87 \\
\hline 20 & 1 & 9 & 295.45 & 204.43 & 139.87 \\
\hline 22 & 1 & 9 & 405.30 & 236.71 & 139.87 \\
\hline 24 & 1 & 9 & 29.52 & 268.99 & 139.87 \\
\hline 26 & 1 & 9 & 2.62 & -32.28 & 166.77 \\
\hline 1 & 2 & 9 & 1491.99 & 1721.53 & 107.60 \\
\hline 3 & 2 & 9 & 52.53 & 177.53 & 112.97 \\
\hline 5 & 2 & 9 & 14606.80 & 13282.70 & 242.09 \\
\hline 7 & 2 & 9 & 2821.79 & 2921.22 & 118.36 \\
\hline 9 & 2 & 9 & 15837.60 & 15778.90 & 295.89 \\
\hline 11 & 2 & 9 & 16858.80 & 16558.90 & 290.51 \\
\hline 13 & 2 & 9 & 657.83 & 435.76 & 96.84 \\
\hline 15 & 2 & 9 & 7755.87 & 7816.81 & 182.91 \\
\hline 17 & 2 & 9 & 599.78 & 516.46 & 102.22 \\
\hline 19 & 2 & 9 & 15.95 & 150.63 & 102.22 \\
\hline 21 & 2 & 9 & 5226.12 & 4664.26 & 182.91 \\
\hline 23 & 2 & 9 & 3011.73 & 3001.91 & 177.53 \\
\hline 25 & 2 & 9 & 1373.04 & 984.50 & 177.53 \\
\hline 0 & 3 & 9 & 3813.94 & 4002.55 & 274.37 \\
\hline 2 & 3 & 9 & 6238.81 & 7181.99 & 285.13 \\
\hline 4 & 3 & 9 & 11428.80 & 11189.90 & 381.96 \\
\hline 6 & 3 & 9 & 918.02 & 1081.33 & 112.97 \\
\hline 8 & 3 & 9 & 666.96 & 441.14 & 112.97 \\
\hline 10 & 3 & 9 & 166.20 & 209.81 & 112.97 \\
\hline 12 & 3 & 9 & 999.05 & 962.98 & 118.36 \\
\hline 14 & 3 & 9 & 83.32 & 96.84 & 102.22 \\
\hline 16 & 3 & 9 & 2.59 & 21.52 & 96.84 \\
\hline 18 & 3 & 9 & 43.36 & -5.38 & 118.36 \\
\hline 20 & 3 & 9 & 1844.13 & 1425.64 & 139.87 \\
\hline 22 & 3 & 9 & 16.94 & 80.70 & 145.25 \\
\hline 24 & 3 & 9 & 44.93 & -32.28 & 139.87 \\
\hline 1 & 4 & 9 & 8272.94 & 7526.30 & 204.43 \\
\hline 3 & 4 & 9 & 11212.80 & 10727.30 & 333.55 \\
\hline 5 & 4 & 9 & 1155.34 & 1043.68 & 156.01 \\
\hline 7 & 4 & 9 & 7451.31 & 6999.08 & 274.37 \\
\hline 9 & 4 & 9 & 19.15 & -129.12 & 150.63 \\
\hline 11 & 4 & 9 & 166.12 & 139.87 & 156.01 \\
\hline 13 & 4 & 9 & 5440.58 & 5369.01 & 193.67 \\
\hline 15 & 4 & 9 & 1502.79 & 2038.93 & 156.01 \\
\hline 17 & 4 & 9 & 6865.31 & 6364.27 & 209.81 \\
\hline 19 & 4 & 9 & 7381.56 & 7058.26 & 220.57 \\
\hline 21 & 4 & 9 & 411.89 & 419.62 & 145.25 \\
\hline 23 & 4 & 9 & 2466.61 & 2587.67 & 150.63 \\
\hline 0 & 5 & 9 & 8.51 & 80.70 & 199.05 \\
\hline 2 & 5 & 9 & 4.95 & 16.14 & 161.39 \\
\hline 4 & 5 & 9 & 428.20 & 451.90 & 204.43 \\
\hline 6 & 5 & 9 & 192.34 & 231.33 & 188.29 \\
\hline 8 & 5 & 9 & 3880.35 & 3093.37 & 215.19 \\
\hline
\end{tabular}




\begin{tabular}{|c|c|c|c|c|c|}
\hline 10 & 5 & 9 & 1956.92 & 2237.98 & 182.910 \\
\hline 12 & 5 & 9 & 2176.93 & 2028.17 & 182.910 \\
\hline 14 & 5 & 9 & 1412.96 & 1205.07 & 177.530 \\
\hline 16 & 5 & 9 & 1868.32 & 1769.94 & 166.770 \\
\hline 18 & 5 & 9 & 901.67 & 866.14 & 161.390 \\
\hline 20 & 5 & 9 & 953.74 & 661.71 & 145.250 \\
\hline 1 & 6 & 9 & 1171.22 & 1081.33 & $161.39 \mathrm{c}$ \\
\hline 3 & 6 & 9 & 33.48 & -91.46 & 182.91 \\
\hline 5 & 6 & 9 & 7845.18 & 6950.66 & 258.23 \\
\hline 7 & 6 & 9 & 1968.11 & 1942.10 & 172.15 \\
\hline 9 & 6 & 9 & 9386.04 & 9156.37 & 290.51 \\
\hline 11 & 6 & 9 & 10096.00 & 9995.62 & 312.03 \\
\hline 13 & 6 & 9 & 254.22 & 242.09 & 161.39 \\
\hline 15 & 6 & 9 & 3126.91 & 3206.34 & 193.67 \\
\hline 0 & 7 & 9 & 502.33 & 312.03 & $290.51<$ \\
\hline 2 & 7 & 9 & 550.16 & 150.63 & $236.71<$ \\
\hline 4 & 7 & 9 & 626.54 & 688.61 & 193.670 \\
\hline 6 & 7 & 9 & 91.36 & 21.52 & $199.05<$ \\
\hline 8 & 7 & 9 & 108.57 & 392.72 & 193.67 \\
\hline 10 & 7 & 9 & 100.38 & 193.67 & 199.05 \\
\hline 0 & 0 & 10 & 37553.70 & 33440.70 & 1608.55 \\
\hline 2 & 0 & 10 & 9225.36 & 10313.00 & 629.43 \\
\hline 4 & 0 & 10 & 24.56 & -188.29 & 301.27 \\
\hline 6 & 0 & 10 & 5813.53 & 5019.33 & 306.65 \\
\hline 8 & 0 & 10 & 3866.98 & 4712.68 & 301.27 \\
\hline 10 & 0 & 10 & 6197.73 & 5815.53 & 322.790 \\
\hline 12 & 0 & 10 & 726.85 & 1022.16 & 225.95 \\
\hline 14 & 0 & 10 & 94.92 & 86.08 & 215.19 \\
\hline 16 & 0 & 10 & 50.25 & -10.76 & 220.57 \\
\hline 18 & 0 & 10 & 5537.20 & 4266.16 & 290.51 \\
\hline 20 & 0 & 10 & 10513.40 & 10689.60 & 457.28 \\
\hline 22 & 0 & 10 & 5272.74 & 4766.48 & 312.03 \\
\hline 24 & 0 & 10 & 559.11 & 301.27 & 225.95 \\
\hline 1 & 1 & 10 & 8312.33 & 7666.17 & 263.61 \\
\hline 3 & 1 & 10 & 11026.60 & 11733.30 & 489.56 \\
\hline 5 & 1 & 10 & 1941.39 & 2453.18 & 177.53 \\
\hline 7 & 1 & 10 & 1717.60 & 1560.13 & 161.39 \\
\hline 9 & 1 & 10 & 5047.21 & 4519. & 209.81 \\
\hline 11 & 1 & 10 & 5689.68 & & \\
\hline 13 & 1 & 10 & 1572.32 & 1581.65 & 172.15 \\
\hline 15 & 1 & 10 & 1995.57 & 1974.38 & 177.53 \\
\hline 17 & 1 & 10 & 5124.38 & 4685.78 & 215.19 \\
\hline 19 & 1 & 10 & 5699.79 & 6299.71 & 242.09 \\
\hline 21 & 1 & 10 & 1735.35 & 1431.02 & 172.15 \\
\hline 23 & 1 & 10 & 2790.37 & 2523.11 & 188.29 \\
\hline 0 & 2 & 10 & 4711.06 & 5519.65 & 322.79 \\
\hline 2 & 2 & 10 & 7374.32 & 7262.69 & 365.82 \\
\hline 4 & 2 & 10 & 917.81 & 1032.92 & 231.33 \\
\hline 6 & 2 & 10 & 1566.83 & 2135.77 & 177.53 \\
\hline 8 & 2 & 10 & 5462.94 & 5976.93 & 242.09 \\
\hline 10 & 2 & 10 & 3.66 & 123.74 & 161.39 \\
\hline 12 & 2 & 10 & 3774.07 & 3518.37 & 204.43 \\
\hline 14 & 2 & 10 & 6338.45 & 6606.36 & 252.85 \\
\hline 16 & 2 & 10 & 1254.20 & 1554.75 & 156.01 \\
\hline
\end{tabular}




$\begin{array}{rrrrrrr}18 & 2 & 10 & 1602.14 & 1398.74 & 177.53 & \circ \\ 20 & 2 & 10 & 1213.58 & 1576.27 & 177.53 & \circ \\ 22 & 2 & 10 & 337.81 & 419.62 & 156.01 & < \\ 1 & 3 & 10 & 2826.75 & 2975.01 & 193.67 & \circ \\ 3 & 3 & 10 & 3825.71 & 3701.28 & 295.89 & \circ \\ 5 & 3 & 10 & 2812.51 & 2948.11 & 204.43 & \circ \\ 7 & 3 & 10 & 3338.04 & 3459.19 & 209.81 & \circ \\ 9 & 3 & 10 & 58.04 & 112.97 & 166.77 & < \\ 11 & 3 & 10 & 194.27 & -59.18 & 150.63 & < \\ 13 & 3 & 10 & 3026.68 & 3330.08 & 188.29 & \circ \\ 15 & 3 & 10 & 1875.76 & 1452.54 & 166.77 & \circ \\ 17 & 3 & 10 & 2111.45 & 1931.34 & 150.63 & \circ \\ 19 & 3 & 10 & 4055.82 & 4519.01 & 220.57 & \circ \\ 21 & 3 & 10 & 368.79 & 468.04 & 172.15 & < \\ 0 & 4 & 10 & 9991.67 & 10291.50 & 468.04 & \circ \\ 2 & 4 & 10 & 793.54 & 850.00 & 252.85 & \circ \\ 4 & 4 & 10 & 1740.20 & 2178.81 & 247.47 & \circ \\ 6 & 4 & 10 & 8446.31 & 8500.04 & 252.85 & \circ \\ 8 & 4 & 10 & 5099.36 & 5734.84 & 236.71 & \circ \\ 10 & 4 & 10 & 4654.49 & 4470.59 & 225.95 & \circ \\ 12 & 4 & 10 & 554.05 & 817.72 & 182.91 & \circ \\ 14 & 4 & 10 & 0.44 & -26.90 & 177.53 & < \\ 16 & 4 & 10 & 248.69 & 349.68 & 177.53 & < \\ 18 & 4 & 10 & 1947.03 & 1732.29 & 193.67 & \circ \\ 20 & 4 & 10 & 4714.30 & 4578.19 & 225.95 & \circ \\ 1 & 5 & 10 & 5030.93 & 5358.25 & 274.37 & \circ \\ 3 & 5 & 10 & 6575.34 & 7138.96 & 338.93 & \circ \\ 5 & 5 & 10 & 697.91 & 753.17 & 182.91 & \circ \\ 7 & 5 & 10 & 579.17 & 780.07 & 215.19 & \circ \\ 9 & 5 & 10 & 5037.56 & 4653.50 & 263.61 & \circ \\ 11 & 5 & 10 & 5130.74 & 5282.94 & 242.09 & \circ \\ 13 & 5 & 10 & 676.64 & 1000.64 & 193.67 & \circ \\ 15 & 5 & 10 & 1608.07 & 1108.23 & 193.67 & \circ \\ 17 & 5 & 10 & 1936.73 & 1915.20 & 188.29 & \circ \\ 0 & 6 & 10 & 5268.72 & 5245.28 & 312.03 & \circ \\ 2 & 6 & 10 & 6168.10 & 5369.01 & 317.41 & \circ \\ 4 & 6 & 10 & 1466.11 & 1624.69 & 263.61 & \circ \\ 6 & 6 & 10 & 132.11 & 86.08 & 193.67 & < \\ 8 & 6 & 10 & 1274.08 & 1366.46 & 204.43 & \circ \\ 10 & 6 & 10 & 112.08 & -48.42 & 199.05 & < \\ 12 & 6 & 10 & 1969.20 & 1931.34 & 215.19 & \circ \\ 1 & 0 & 11 & 2817.30 & 2560.77 & 268.99 & \circ \\ 3 & 0 & 11 & 108.57 & -328.17 & 333.55 & < \\ 5 & 0 & 11 & 9986.18 & 8290.23 & 403.48 & \circ \\ 7 & 0 & 11 & 3612.31 & 4395.27 & 312.03 & \circ \\ 9 & 0 & 11 & 8995.93 & 9005.74 & 425.00 & \circ \\ 11 & 0 & 11 & 8779.25 & 8516.18 & 414.24 & \circ \\ 13 & 0 & 11 & 656.59 & 737.03 & 247.47 & < \\ 15 & 0 & 11 & 8495.83 & 7343.39 & 387.34 & \circ \\ 17 & 0 & 11 & 78.72 & 279.75 & 247.47 & < \\ 19 & 0 & 11 & 62.99 & -10.76 & 247.47 & < \\ 0 & 0 & 11 & 4002.69 & 3738.94 & 312.03 & \circ \\ 2 & 1 & 11 & 208.51 & 306.65 & 225.95 & < \\ & & 11 & 3089.55 & 3055.71 & 279.75 & \circ \\ & & & & & \end{array}$




$\begin{array}{rrrrrrr}4 & 1 & 11 & 12193.10 & 10845.60 & 484.18 & \circ \\ 6 & 1 & 11 & 2255.04 & 2539.25 & 193.67 & \circ \\ 8 & 1 & 11 & 328.82 & 263.61 & 172.15 & < \\ 10 & 1 & 11 & 489.53 & 586.40 & 172.15 & \circ \\ 12 & 1 & 11 & 2170.66 & 2646.85 & 204.43 & \circ \\ 14 & 1 & 11 & 17.28 & -10.76 & 172.15 & < \\ 16 & 1 & 11 & 1237.62 & 1549.37 & 188.29 & \circ \\ 18 & 1 & 11 & 8.97 & -279.75 & 177.53 & < \\ 20 & 1 & 11 & 2005.88 & 1759.19 & 199.05 & \circ \\ 1 & 2 & 11 & 5733.21 & 4954.77 & 236.71 & \circ \\ 3 & 2 & 11 & 8826.20 & 7988.96 & 414.24 & \circ \\ 5 & 2 & 11 & 753.08 & 785.45 & 182.91 & \circ \\ 7 & 2 & 11 & 4827.75 & 5498.13 & 247.47 & \circ \\ 9 & 2 & 11 & 1907.88 & 1522.48 & 193.67 & \circ \\ 11 & 2 & 11 & 2025.74 & 1656.97 & 193.67 & \circ \\ 13 & 2 & 11 & 6758.83 & 6568.70 & 263.61 & \circ \\ 15 & 2 & 11 & 2218.52 & 2926.60 & 209.81 & \circ \\ 17 & 2 & 11 & 3268.64 & 2862.04 & 215.19 & \circ \\ 19 & 2 & 11 & 4602.27 & 4126.28 & 231.33 & \circ \\ 0 & 3 & 11 & 32.93 & -107.60 & 263.61 & < \\ 2 & 3 & 11 & 652.95 & 570.26 & 252.85 & < \\ 4 & 3 & 11 & 5653.77 & 5745.60 & 371.20 & \circ \\ 6 & 3 & 11 & 2598.69 & 3410.78 & 225.95 & \circ \\ 8 & 3 & 11 & 2603.58 & 2576.91 & 215.19 & \circ \\ 10 & 3 & 11 & 2334.27 & 2926.60 & 220.57 & \circ \\ 12 & 3 & 11 & 3889.48 & 4260.78 & 242.09 & \circ \\ 14 & 3 & 11 & 571.99 & 532.60 & 193.67 & < \\ 16 & 3 & 11 & 2.31 & -156.01 & 193.67 & < \\ 18 & 3 & 11 & 285.87 & 338.93 & 215.19 & < \\ 1 & 4 & 11 & 1086.56 & 1253.49 & 204.430 & \circ \\ 3 & 4 & 11 & 13.19 & 96.84 & 279.75 & < \\ 5 & 4 & 11 & 4880.51 & 4545.91 & 231.33 & \circ \\ 7 & 4 & 11 & 968.82 & 1258.87 & 182.91 & \circ \\ 9 & 4 & 11 & 6638.53 & 6165.22 & 301.27 & \circ \\ 11 & 4 & 11 & 7135.79 & 6563.32 & 306.65 & \circ \\ 13 & 4 & 11 & 34.31 & 220.57 & 193.67 & < \\ 15 & 4 & 11 & 3184.18 & 2716.78 & 220.57 & \circ \\ 0 & 5 & 11 & 5.79 & -236.71 & 322.79 & < \\ 2 & 5 & 11 & 985.88 & 570.26 & 328.17 & < \\ 4 & 5 & 11 & 4381.12 & 3674.38 & 387.34 & \circ \\ 6 & 5 & 11 & 179.37 & 285.13 & 236.71 & < \\ 8 & 5 & 11 & 296.71 & 581.02 & 231.33 & < \\ 10 & 5 & 11 & 14.00 & 10.76 & 204.43 & < \\ 12 & 5 & 11 & 582.65 & 581.02 & 209.81 & < \\ 1 & 6 & 11 & 3812.58 & 3426.91 & 285.13 & \circ \\ 3 & 6 & 11 & 6879.49 & 6245.91 & 451.90 & \circ \\ 0 & 0 & 12 & 513.98 & 312.03 & 355.07 & < \\ 2 & 0 & 12 & 4134.92 & 3518.37 & 446.52 & \circ \\ 4 & 0 & 12 & 2700.08 & 3362.36 & 451.90 & \circ \\ 6 & 0 & 12 & 1812.34 & 2706.02 & 295.89 & \circ \\ 14 & 0 & 12 & 12144.30 & 10555.10 & 489.56 & \circ \\ & 0 & 12 & 4288.18 & 3943.37 & 333.55 & \circ\end{array}$




$\begin{array}{rrrrrrr}16 & 0 & 12 & 35.40 & -112.97 & 274.37 & < \\ 18 & 0 & 12 & 1078.25 & 1043.68 & 285.13 & \circ \\ 1 & 1 & 12 & 2439.85 & 2657.61 & 215.19 & \circ \\ 3 & 1 & 12 & 2782.01 & 2291.78 & 290.51 & \circ \\ 5 & 1 & 12 & 939.59 & 1215.83 & 199.05 & \circ \\ 7 & 1 & 12 & 661.26 & 441.14 & 193.67 & < \\ 9 & 1 & 12 & 2196.75 & 2264.88 & 209.81 & \circ \\ 11 & 1 & 12 & 3140.51 & 2657.61 & 220.57 & \circ \\ 13 & 1 & 12 & 80.93 & 166.77 & 193.67 & < \\ 15 & 1 & 12 & 364.29 & 629.43 & 199.05 & \circ \\ 17 & 1 & 12 & 2339.20 & 2044.31 & 215.19 & \circ \\ 0 & 2 & 12 & 7154.95 & 6756.99 & 403.48 & \circ \\ 2 & 2 & 12 & 776.72 & 704.75 & 279.75 & < \\ 4 & 2 & 12 & 3310.13 & 3674.38 & 338.93 & \circ \\ 6 & 2 & 12 & 5048.04 & 5180.72 & 258.23 & \circ \\ 8 & 2 & 12 & 1828.36 & 2840.52 & 231.33 & \circ \\ 10 & 2 & 12 & 2822.25 & 2636.09 & 220.57 & \circ \\ 12 & 2 & 12 & 2075.30 & 2038.93 & 215.19 & \circ \\ 14 & 2 & 12 & 3.29 & -333.55 & 204.43 & < \\ 16 & 2 & 12 & 297.67 & 516.46 & 204.43 & < \\ 1 & 3 & 12 & 1939.71 & 1786.08 & 225.95 & \circ \\ 3 & 3 & 12 & 2479.77 & 2727.54 & 333.55 & \circ \\ 5 & 3 & 12 & 408.66 & 801.59 & 215.19 & \circ \\ 7 & 3 & 12 & 62.50 & 182.91 & 209.81 & < \\ 9 & 3 & 12 & 3259.94 & 3238.62 & 242.09 & \circ \\ 11 & 3 & 12 & 4039.73 & 4718.06 & 263.61 & \circ \\ 13 & 3 & 12 & 8.29 & 96.84 & 209.81 & < \\ 0 & 4 & 12 & 2692.02 & 3044.95 & 344.30 & \circ \\ 2 & 4 & 12 & 4874.27 & 4524.39 & 371.20 & \circ \\ 4 & 4 & 12 & 2322.65 & 2732.92 & 279.75 & \circ \\ 6 & 4 & 12 & 465.39 & 747.79 & 193.67 & \circ \\ 8 & 4 & 12 & 4401.79 & 4960.15 & 242.09 & \circ \\ 10 & 4 & 12 & 5.15 & 172.15 & 247.47 & < \\ 1 & 5 & 12 & 933.79 & 866.14 & 258.23 & \circ \\ 1 & 0 & 13 & 3148.23 & 2254.12 & 317.41 & \circ \\ 3 & 0 & 13 & 4350.35 & 2905.08 & 457.28 & \circ \\ 5 & 0 & 13 & 89.56 & 419.62 & 295.89 & < \\ 7 & 0 & 13 & 3312.63 & 3238.62 & 338.93 & \circ \\ 9 & 0 & 13 & 4129.84 & 4142.42 & 365.82 & \circ \\ 11 & 0 & 13 & 4903.26 & 3577.55 & 349.68 & \circ \\ 13 & 0 & 13 & 5630.93 & 5385.15 & 392.72 & \circ \\ 0 & 1 & 13 & 599.79 & 204.43 & 295.89 & < \\ 2 & 1 & 13 & 518.74 & 161.39 & 306.65 & < \\ 4 & 1 & 13 & 5045.09 & 5164.58 & 312.03 & \circ \\ 6 & 1 & 13 & 565.54 & 634.81 & 215.19 & < \\ 8 & 1 & 13 & 488.08 & 581.02 & 215.19 & < \\ 10 & 1 & 13 & 657.84 & 613.29 & 220.57 & < \\ 1 & 1 & 13 & 5674.23 & 5358.25 & 279.75 & \circ \\ 3 & 2 & 13 & 421.66 & 543.36 & 252.85 & < \\ 5 & 2 & 13 & 4515.41 & 3652.86 & 258.23 & \circ \\ 7 & 2 & 13 & 1126.36 & 1140.51 & 225.95 & \circ \\ 9 & 2 & 13 & 1478.15 & 1334.18 & 231.33 & \circ \\ 0 & 3 & 13 & 286.75 & 505.70 & 322.79 & < \\ & & & & & & \end{array}$


$\begin{array}{lllllll}2 & 3 & 13 & 1872.46 & 2189.57 & 355.07 & 0\end{array}$

$\begin{array}{lllllll}4 & 3 & 13 & 4421.40 & 4147.80 & 274.37 & 0\end{array}$

$\begin{array}{llllll}6 & 3 & 13 & 305.83 & 333.55 & 236.71<\end{array}$

loop

_twin_refln_datum_id

_twin_refln_individual_id

_twin_refln_index_h

_twin_refln_index_k

_twin_refln_index_l

_twin_refln_F_squared_calc

_twin_refln_F_squared_meas

_twin_refln_F_squared_sigma

_twin_refln_include_status

? ? ? ? ? ? ? ? ? 